Supporting Information for:

\title{
Palladium-Catalyzed Markovnikov \\ Hydroaminocarbonylation of 1,1-Disubstituted and \\ 1,1,2-Trisubstituted Alkenes for Formation of Amides with Quaternary Carbon
}

\begin{abstract}
Hui-Yi Yang, Ya-Hong Yao, Ming Chen, Zhi-Hui Ren, Zheng-Hui Guan*
Key Laboratory of Synthetic and Natural Molecule of Ministry of Education, Department of Chemistry \& Materials Science, Northwest University, Xi'an 710127, P. R. China
\end{abstract}

Email: guanzhh@nwu.edu.cn

\section{CONTENTS}

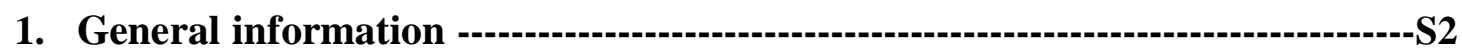

2. Preparation of di-, trisubstituted alkenes---:---S3

3. Typical procedure for the Palladium-catalyzed Markovnikov

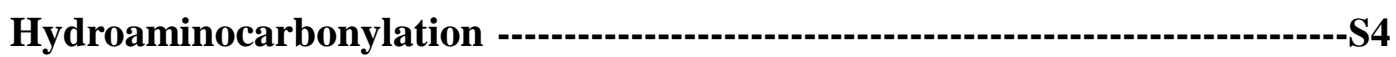

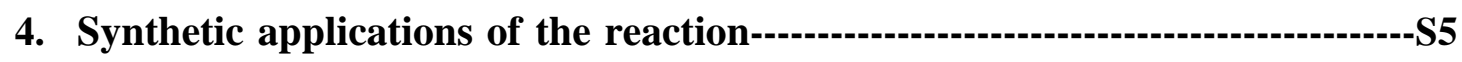

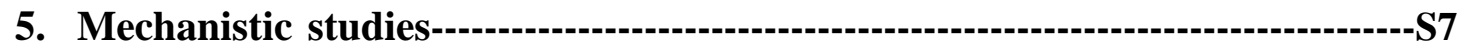

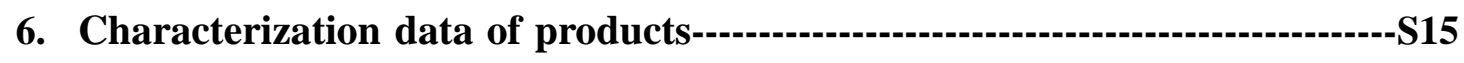

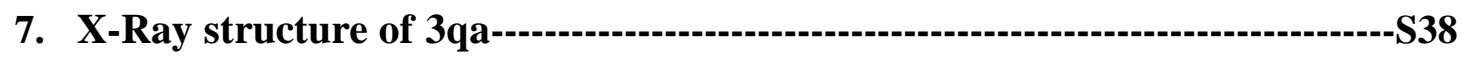

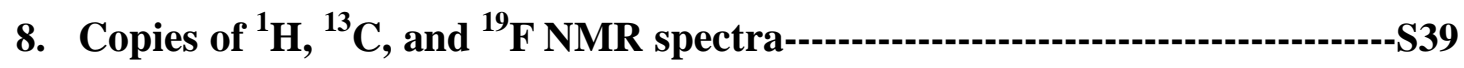




\section{General information.}

\section{Chemicals}

Chemicals were commercially purchased from Adamas-beta, Energy Chemical, Aladdin, etc, and directly used without further purification unless otherwise stated. $\mathrm{PdCl}_{2}, \mathrm{PdBr}_{2}, \mathrm{PdI}_{2}, \mathrm{Pd}\left(\mathrm{PPh}_{3}\right)_{4}$ and $\mathrm{Pd}\left(\mathrm{PPh}_{3}\right)_{2} \mathrm{Cl}_{2}$ were purchased from Adamas-beta. THF used in the reaction was purchased from Shanghai Titan Scientific Co., Ltd. $\left(\mathrm{H}_{2} \mathrm{O} \leqslant 0.1 \%\right)$ and without further purification. Anhydrous THF used in mechanistic studies was distilled from sodium/benzophenone until the indicator had turned a persistent blue color. There is still contained $43 \mathrm{ppm}$ of water in anhydrous THF according to J. Org. Chem. 2010, 75, 8351-8354.

\section{Chromatography}

Analytical thin-layer chromatography (TLC) was carried out with silica gel pre-coated glass plates (TLC-Silica gel GF254, coating thickness: 0.20-0.25 mm, particle size: 10-40 $\mu \mathrm{m}$ ) purchased from Xinnuo Chemical (Yantai, China). The TLC was visualized with a UV lamp (254 or $365 \mathrm{~nm}$ ). Flash Column chromatography was carried out on silica gel (60 ^, 200-300 mesh) purchased from Xinnuo Chemicals (Yantai, China) with technical grade solvents as the eluent. All the yields referred to spectroscopically and chromatographically pure compounds.

\section{Nuclear Magnetic Resonance (NMR) Spectroscopy}

${ }^{1} \mathrm{H}$ NMR spectra were recorded on Bruker AVANCE III-400 instrument (400 $\mathrm{MHz}$ spectrometer). The analytical sample was dissolved in an appropriate deuterated solvent. The employed deuterated solvent and the measuring frequency are indicated in each ${ }^{1} \mathrm{H}$ NMR data. Chemical shifts are reported in parts per million (ppm) with the solvent resonance as the internal reference $\left(\mathrm{CDCl}_{3} \delta 7.26, d^{6}\right.$-DMSO $\left.\delta 2.50\right)$. The following abbreviations (or combinations thereof) were used to explain multiplicities: $\mathrm{s}=$ singlet, $\mathrm{d}=$ doublet, $\mathrm{t}=$ triplet, $\mathrm{q}=$ quartet, $\mathrm{m}=$ multiplet, $\mathrm{b}=$ broad . Coupling constants, J were reported in Hertz unit (Hz).

${ }^{13} \mathrm{C}$ NMR spectra were recorded on Bruker AVANCE III - 400 instrument (101 $\mathrm{MHz}$ spectrometer). The employed deuterated solvent and the measuring frequency are both indicated in each ${ }^{13} \mathrm{C}$ NMR data. Chemical shifts are reported in ppm with the solvent resonance as the internal reference $\left(\mathrm{CDCl}_{3} \delta 77.16\right) .{ }^{19} \mathrm{~F}$ NMR data were recorded on Bruker AVANCE III - 400 instrument (376 MHz spectrometer). 


\section{High Resolution Mass Spectrometry (HRMS)}

HRMS were recorded on a liquid chromatography/quadrupole time-of-flight mass spectrometer (MicroTof-Q II mass spectrometer, Bruker Daltonics) using electrospray ionization-time of flight (ESI-TOF) at Instrumental Analysis Center of Northwest University. The calculated values are based on the most abundant isotope.

\section{X-ray crystallography}

X-ray crystallography was performed on a BRUKERSMA RTAPEXIICCD diffractometer at Instrumental Analysis Center of Northwest University.

\section{Preparation of 1,1-disubstituted alkenes, and 1,1,2-trisubstituted alkenes}

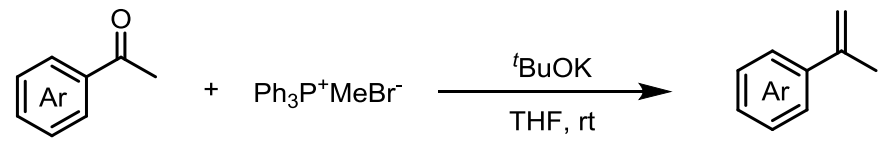

To a $25 \mathrm{ml}$ round bottomed flask were added methyl triphenylphosphonium bromide ( $7.5 \mathrm{mmol}, 1.5$ equiv) and ${ }^{t} \mathrm{BuOK}$ ( $7.5 \mathrm{mmol}, 1.5$ equiv). Adding $10 \mathrm{~mL}$ of dry THF under argon, the mixture was stirred at room temperature for 1 hour. After that diluted ketone ( $5 \mathrm{mmol}, 1.0$ equiv) in dry THF (3 mL) was added, then the reaction was stirred at room temperature for overnight. The mixture was diluted with $\mathrm{CH}_{2} \mathrm{Cl}_{2}(25 \mathrm{~mL})$, washed with brine $(3 \times 15 \mathrm{~mL})$, dried with $\mathrm{Na}_{2} \mathrm{SO}_{4}$, filtered and concentrated in vacuum. The crude material was purified by column chromatography (hexanes as the eluent) to afford the 1,1-disubstituted alkenes.

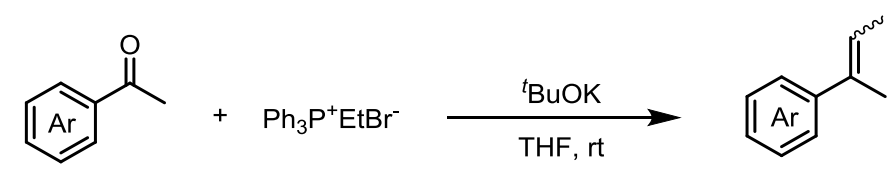

To a $25 \mathrm{ml}$ round bottomed flask were added ethyl triphenylphosphonium bromide (7.5 mmol, 1.5 equiv) and ${ }^{t} \mathrm{BuOK}$ (7.5 mmol, 1.5 equiv). Adding $10 \mathrm{~mL}$ of dry THF under argon, the mixture was stirred at room temperature for 1 hour. After that diluted ketone ( $5 \mathrm{mmol}, 1.0$ equiv) in dry THF $(3 \mathrm{~mL})$ was added, then the reaction was stirred at room temperature for overnight. The mixture was diluted with $\mathrm{CH}_{2} \mathrm{Cl}_{2}(25 \mathrm{~mL})$, washed with brine $(3 \times 15 \mathrm{~mL})$, dried with $\mathrm{Na}_{2} \mathrm{SO}_{4}$, filtered and concentrated in vacuum. The crude material was purified by column chromatography (hexanes as the eluent) to afford the 1,1,2-trisubstituted alkenes. 


\section{Typical procedure for the palladium-catalyzed Markovnikov}

hydroaminocarbonylation

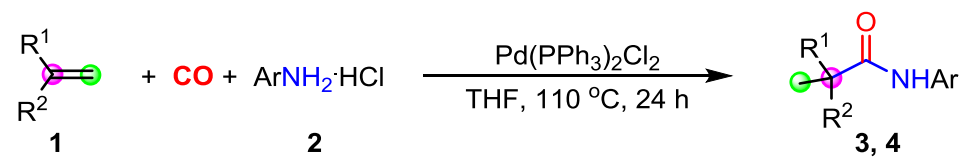

For Table 2: A mixture of 1,1-disubstituted alkenes 1 ( $0.24 \mathrm{mmol}, 1.2$ equiv), aniline hydrochloride salts 2 (0.2 mmol, 1.0 equiv), $\mathrm{Pd}\left(\mathrm{PPh}_{3}\right)_{2} \mathrm{Cl}_{2}(0.006 \mathrm{mmol}, 3$ mol\%), and THF (1.2 mL) were added into a glass tube which was placed in an autoclave. The autoclave was evacuated and backfilled with $\mathrm{CO}$ for three times in a well-ventilated fume hood, and then pressurized to $45 \mathrm{~atm}$ of $\mathrm{CO}$. The reaction mixture in autoclave was stirred at $110^{\circ} \mathrm{C}$ for 24 hours. After that, the autoclave was removed from the oil bath and cooled to room temperature prior to the release of excess carbon monoxide. The regioselectivities were determined by GC-MS analysis of the crude products. Then the corresponding reaction mixture was purified by flash column chromatography on a silica gel column (petroleum ether/ethyl acetate $=10 / 1$ ) to give the product 3 and $\mathbf{4}$.

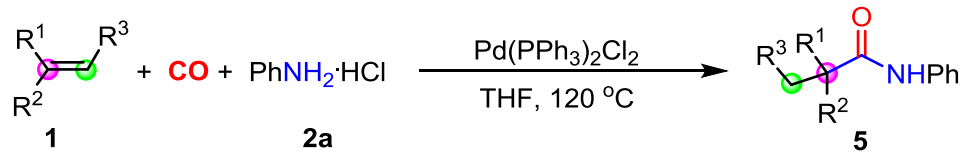

For Table 3: A mixture of 1,1,2-trisubstituted alkenes 1 ( $0.24 \mathrm{mmol}, 1.2$ equiv), aniline hydrochloride salt 2a $(0.2 \mathrm{mmol}, 1.0$ equiv $), \mathrm{Pd}\left(\mathrm{PPh}_{3}\right)_{2} \mathrm{Cl}_{2}(0.006 \mathrm{mmol}, 3$ mol\%), and THF $(0.3 \mathrm{~mL})$ were added into a glass tube which was placed in an autoclave. The autoclave was evacuated and backfilled with $\mathrm{CO}$ for three times in a well-ventilated fume hood, and then pressurized to $65 \mathrm{~atm}$ of $\mathrm{CO}$. The reaction mixture in autoclave was reacted at $120{ }^{\circ} \mathrm{C}$ for $96-144$ hours without stirring. After that, the autoclave was removed from the oil bath and cooled to room temperature prior to the release of excess carbon monoxide. The regioselectivities were determined by GC-MS analysis of the crude products. Then the corresponding reaction mixture was purified by flash column chromatography on a silica gel column (petroleum ether/ethyl acetate $=10 / 1)$ to give the products $\mathbf{5}$. 
Notably, we found that the stirring played an important role in the reaction. Only trace of product was observed when the reaction was stirring at $500 \mathrm{rpm}$ (lots of palladium black formed, as following Figure, right). However, the reaction goes well when it was performed without stirring (as following Figure, left). The detailed mechanism remains unclear.

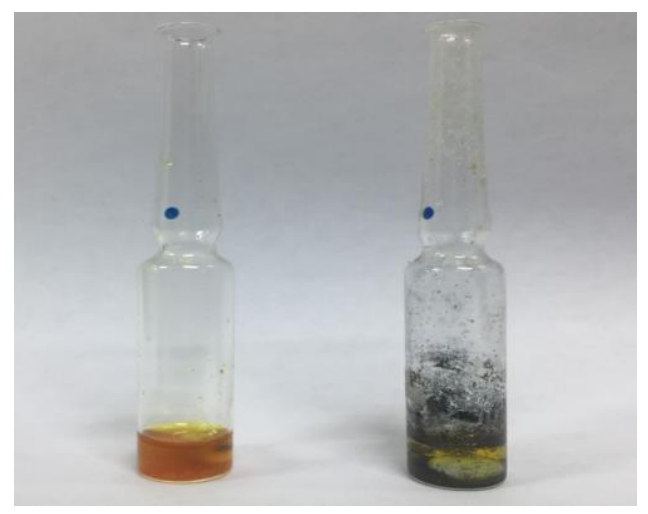

For left reaction, without stirring; for right reaction, stirring at $500 \mathrm{rpm}$

\section{Synthetic applications of the reaction}

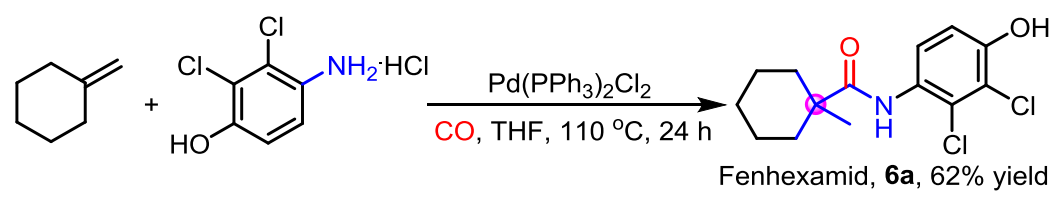

A mixture of 1-methylenecyclohexane (0.24 mmol, 1.2 equiv), 2,3-dichloro-4-aminophenol hydrochloride (0.2 mmol, 1.0 equiv), $\mathrm{Pd}\left(\mathrm{PPh}_{3}\right)_{2} \mathrm{Cl}_{2}$ (0.006 mmol, $3 \mathrm{~mol} \%)$, and THF (1.2 mL) were added into a glass tube which was placed in an autoclave. The autoclave was evacuated and backfilled with CO for three times in a well-ventilated fume hood, and then pressurized to $45 \mathrm{~atm}$ of $\mathrm{CO}$. The reaction mixture in autoclave was stirred at $110{ }^{\circ} \mathrm{C}$ for 24 hours. After that, the autoclave was removed from the oil bath and cooled to room temperature prior to the release of excess carbon monoxide. The regioselectivity $(b / 1=95: 5)$ were determined by GC-MS analysis of the crude products. Then the corresponding reaction mixture was purified by flash column chromatography on a silica gel column (petroleum ether/ethyl acetate $=6 / 1$ ) to give the fenhexamid $\mathbf{6 a}$ in $62 \%$ yield. 


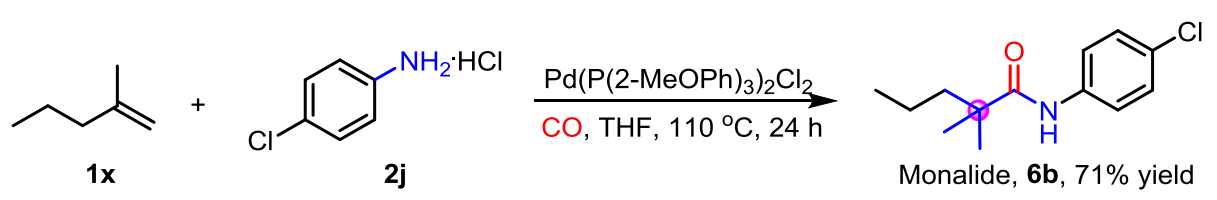

A mixture of $\mathbf{1 x}(0.24 \mathrm{mmol}, 1.2$ equiv $), \mathbf{2 j}(0.2 \mathrm{mmol}, 1.0$ equiv), $\mathrm{Pd}\left(\mathrm{P}(2-\mathrm{MeOPh})_{3}\right)_{2} \mathrm{Cl}_{2}(0.006 \mathrm{mmol}, 3 \mathrm{~mol} \%)$, and THF $(1.2 \mathrm{~mL})$ were added into a glass tube which was placed in an autoclave. The autoclave was evacuated and backfilled with $\mathrm{CO}$ for three times in a well-ventilated fume hood, and then pressurized to $45 \mathrm{~atm}$ of $\mathrm{CO}$. The reaction mixture in autoclave was stirred at $110^{\circ} \mathrm{C}$ for 24 hours. After that, the autoclave was removed from the oil bath and cooled to room temperature prior to the release of excess carbon monoxide. The regioselectivity (b:1=95:5) were determined by GC-MS analysis of the crude products. Then the corresponding reaction mixture was purified by flash column chromatography on a silica gel column (petroleum ether/ethyl acetate $=10 / 1$ ) to give the $\mathbf{6 b}$ in $71 \%$ yield.

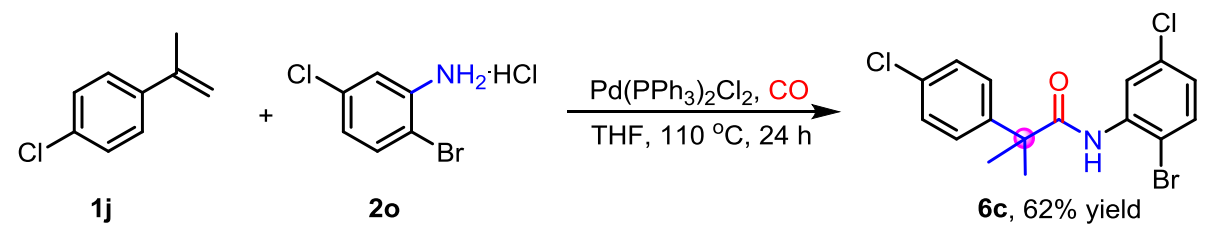

A mixture of $\mathbf{1 j}$ (0.24 mmol, 1.2 equiv), $2 \mathbf{o}$ ( $0.2 \mathrm{mmol}, 1.0$ equiv), $\mathrm{Pd}\left(\mathrm{PPh}_{3}\right)_{2} \mathrm{Cl}_{2}$ (0.006 mmol, $3 \mathrm{~mol} \%)$, and THF $(1.2 \mathrm{~mL})$ were added into a glass tube which was placed in an autoclave. The autoclave was evacuated and backfilled with CO for three times in a well-ventilated fume hood, and then pressurized to $45 \mathrm{~atm}$ of CO. The reaction mixture in autoclave was stirred at $110{ }^{\circ} \mathrm{C}$ for 24 hours. After that, the autoclave was removed from the oil bath and cooled to room temperature prior to the release of excess carbon monoxide. The regioselectivity $(b / 1=97: 3)$ were determined by GC-MS analysis of the crude products. Then the corresponding reaction mixture was purified by flash column chromatography on a silica gel column (petroleum ether/ethyl acetate $=10 / 1)$ to give the $\mathbf{6 c}$ in $62 \%$ yield. 


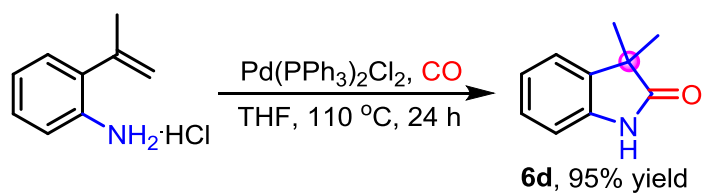

A mixture of 2-(prop-1-en-2-yl)aniline hydrochloride ( $0.20 \mathrm{mmol}, 1.0$ equiv), $\mathrm{Pd}\left(\mathrm{PPh}_{3}\right)_{2} \mathrm{Cl}_{2}(0.006 \mathrm{mmol}, 3 \mathrm{~mol} \%)$, and THF $(1.2 \mathrm{~mL})$ were added into a glass tube which was placed in an autoclave. The autoclave was evacuated and backfilled with $\mathrm{CO}$ for three times in a well-ventilated fume hood, and then pressurized to 45 atm of CO. The reaction mixture in autoclave was stirred at $110{ }^{\circ} \mathrm{C}$ for 24 hours. After that, the autoclave was removed from the oil bath and cooled to room temperature prior to the release of excess carbon monoxide. The regioselectivity (b:1>99:1) were determined by GC-MS analysis of the crude products. Then the corresponding reaction mixture was purified by flash column chromatography on a silica gel column (petroleum ether/ethyl acetate $=10 / 1$ ) to give the $\mathbf{6} \mathbf{d}$ in $95 \%$ yield.

\section{Mechanistic studies}

\subsection{Control experiments (Scheme 3 in maintext)}

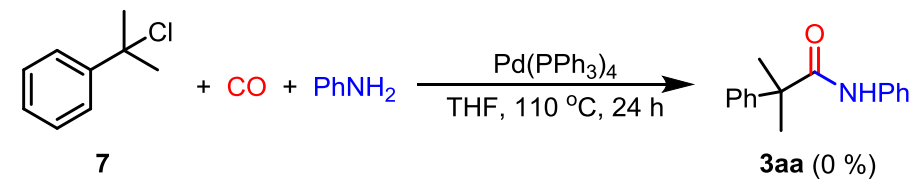

A mixture of (2-chloropropan-2-yl)benzene 7 (0.24 mmol, 1.2 equiv), aniline (0.2 mmol, 1.0 equiv), $\mathrm{Pd}\left(\mathrm{PPh}_{3}\right)_{4}(0.01 \mathrm{mmol}, 5 \mathrm{~mol} \%)$, and THF (1.2 mL) were added into a glass tube which was placed in an autoclave. The autoclave was evacuated and backfilled with $\mathrm{CO}$ for three times in a well-ventilated fume hood, and then pressurized to $45 \mathrm{~atm}$ of $\mathrm{CO}$. The reaction mixture in autoclave was stirred at 110 ${ }^{\circ} \mathrm{C}$ for 24 hours. After that, the autoclave was removed from the oil bath and cooled to room temperature prior to the release of excess carbon monoxide. No desired 3aa was detected by GC-MS.

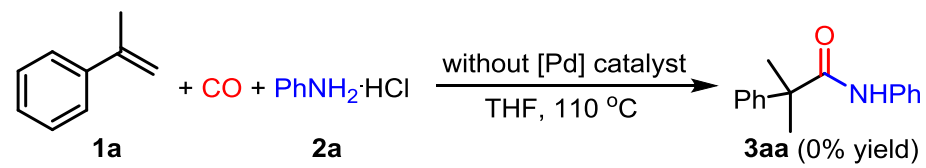


A mixture of $\alpha$-methyl styrene $1 \mathbf{a}(0.24 \mathrm{mmol}, 1.2$ equiv), aniline hydrochloride salt $2 \mathrm{a}(0.2 \mathrm{mmol}, 1.0$ equiv), THF $(1.2 \mathrm{~mL})$ were added into a glass tube which was placed in an autoclave. The autoclave was evacuated and backfilled with $\mathrm{CO}$ for three times in a well-ventilated fume hood, and then pressurized to $45 \mathrm{~atm}$ of $\mathrm{CO}$. The reaction mixture in autoclave was stirred at $110{ }^{\circ} \mathrm{C}$ for 24 hours. After that, the autoclave was removed from the oil bath and cooled to room temperature prior to the release of excess carbon monoxide. No desired 3aa was detected by GC-MS.

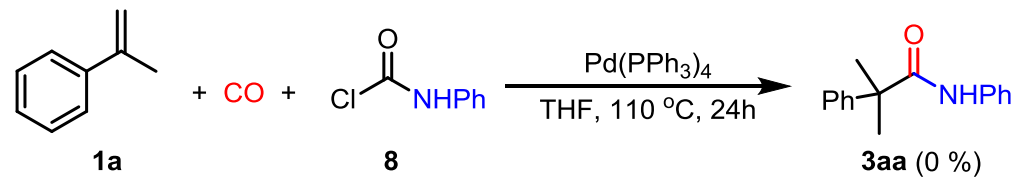

A mixture of $\alpha$-methyl styrene $1 \mathrm{a}(0.24 \mathrm{mmol}, 1.2$ equiv), phenylcarbamic chloride 8 (0.2 mmol, 1.0 equiv), $\mathrm{Pd}\left(\mathrm{PPh}_{3}\right)_{4}(0.01 \mathrm{mmol}, 5 \mathrm{~mol} \%)$, and THF (1.2 mL) were added into a glass tube which was placed in an autoclave. The autoclave was evacuated and backfilled with CO for three times in a well-ventilated fume hood, and then pressurized to $45 \mathrm{~atm}$ of $\mathrm{CO}$. The reaction mixture in autoclave was stirred at 110 ${ }^{\circ} \mathrm{C}$ for 24 hours. After that, the autoclave was removed from the oil bath and cooled to room temperature prior to the release of excess carbon monoxide. No desired 3aa was detected by GC-MS.

\subsection{Anhydrous studies}

Anhydrious THF was distilled from sodium/benzophenone once the indicator had turned a persistent blue color. There is still contained $43 \mathrm{ppm}$ of water in anhydrous THF according to $J$. Org. Chem. 2010, 75, 8351-8354. Before they were used in the reaction, anilines hydrochloride, palladium catalyst, glass tube and autoclave were dried in a drying box at $120{ }^{\circ} \mathrm{C}$ for $3 \mathrm{~h}$.

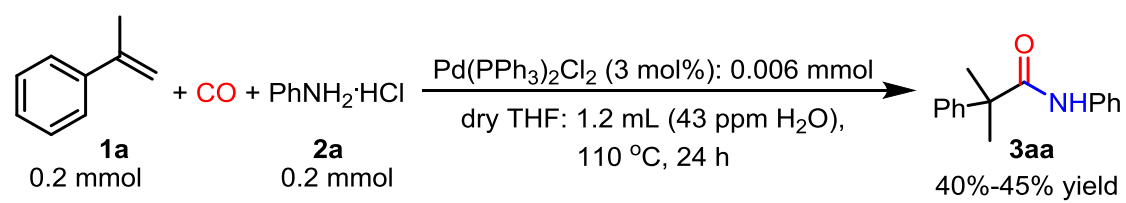


A mixture of $1 \mathbf{a}\left(0.24 \mathrm{mmol}, 1.2\right.$ equiv), $2 \mathrm{a}\left(0.2 \mathrm{mmol}, 1.0\right.$ equiv), $\mathrm{Pd}\left(\mathrm{PPh}_{3}\right)_{2} \mathrm{Cl}_{2}$ (0.006 mmol, $3 \mathrm{~mol} \%)$, and anhydrious THF $(1.2 \mathrm{~mL})$ were added into a glass tube which was placed in an autoclave. The autoclave was evacuated and backfilled with $\mathrm{CO}$ for three times in a well-ventilated fume hood, and then pressurized to 45 atm of CO. The reaction mixture in autoclave was stirred at $110{ }^{\circ} \mathrm{C}$ for 24 hours. After that, the autoclave was removed from the oil bath and cooled to room temperature prior to the release of excess carbon monoxide. Then the corresponding reaction mixture was purified by flash column chromatography on a silica gel column (petroleum ether/ethyl acetate $=10 / 1)$ to give the product 3aa.

Above anhydrous reaction was run 3 times, 40-45\% yield of 3aa was obtained. There is still $0.003 \mathrm{mmol}$ water in $1.2 \mathrm{~mL}$ anhydrous THF (43 ppm), which is 0.5 equiv for $\mathrm{Pd}\left(\mathrm{PPh}_{3}\right)_{2} \mathrm{Cl}_{2}$ catalyst (3 mol\%). Further control experiment in the presence of $1 \mathrm{~mol} \%$ of $\mathrm{Pd}\left(\mathrm{PPh}_{3}\right)_{2} \mathrm{Cl}_{2}$ catalyst in $1.2 \mathrm{~mL}$ commercially purchased THF resulted in $86 \%$ yield of 3aa. These experiments suggested water played an important role in the reaction.

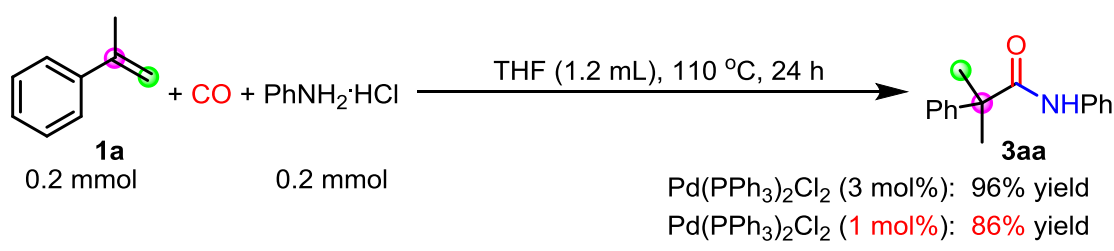

\subsection{Deuterium-Labeling studies}

\subsubsection{Preparation of $1 \mathrm{q}-\mathrm{D}_{5}$}

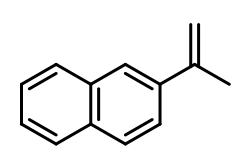

$1 q$

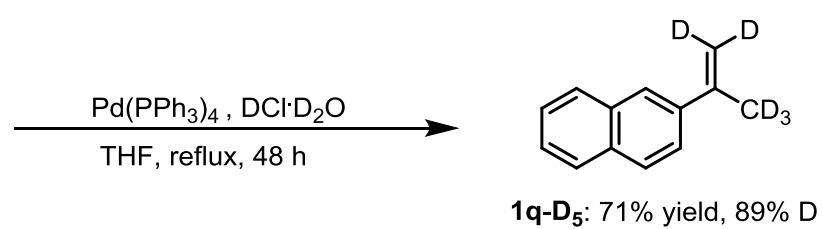

1q-D: $\mathrm{D}_{5}: 71 \%$ yield, $89 \% \mathrm{D}$

A mixture of 2-isopropenylnaphthalene 1q ( $0.2 \mathrm{mmol}, 1.0$ equiv), $\mathrm{DCl} \mathrm{D}_{2} \mathrm{O}$ (93.6 mg, 20.0 equiv), $\mathrm{Pd}\left(\mathrm{PPh}_{3}\right)_{4}(0.006 \mathrm{mmol}, 3 \mathrm{~mol} \%)$, and anhydrous THF $(1.2 \mathrm{~mL})$ were added into a round bottomed flask which was reflux under argon in an oil bath 
for 48 hours. After completion, the reaction mixture was purified by flash column chromatography on a silica gel column (petroleum ether/ethyl acetate $=100 / 1$ ) to give the 1q-D $\mathbf{D}_{\mathbf{5}}$ in $71 \%$ yield with $89 \%$ D.

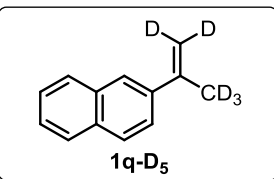

1q-D5: ${ }^{1} \mathrm{H}$ NMR (400 MHz, $d^{6}$-DMSO) $\delta$ 7.96-7.86 (m, 4H), $7.75(\mathrm{dd}, J=8.8,1.4 \mathrm{~Hz}$, $1 \mathrm{H}), 7.52-7.47(\mathrm{~m}, 2 \mathrm{H}), 5.61(\mathrm{~d}, J=6.0 \mathrm{~Hz}, 0.12 \mathrm{H}), 5.21(\mathrm{~d}, J=5.2 \mathrm{~Hz}, 0.11 \mathrm{H})$, $2.19(\mathrm{~s}, 0.33 \mathrm{H})$.

${ }^{1} \mathrm{H}$ NMR (400 MHz, $d^{6}$-DMSO) of compound $\mathbf{1 q - \mathbf { D } _ { 5 }}$
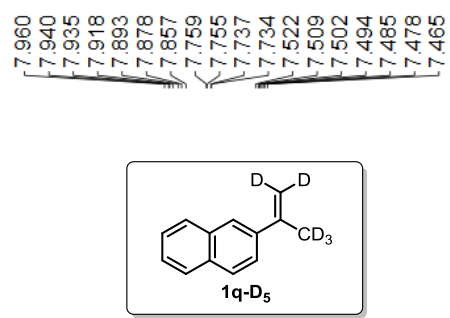

$\mathrm{D} \%=1-\frac{\frac{0.12+0.11+0.33}{1+1+3}}{\frac{4.08+1.00+2.01}{4+1+2}}=1-\frac{0.112}{1.013}=89 \%$.

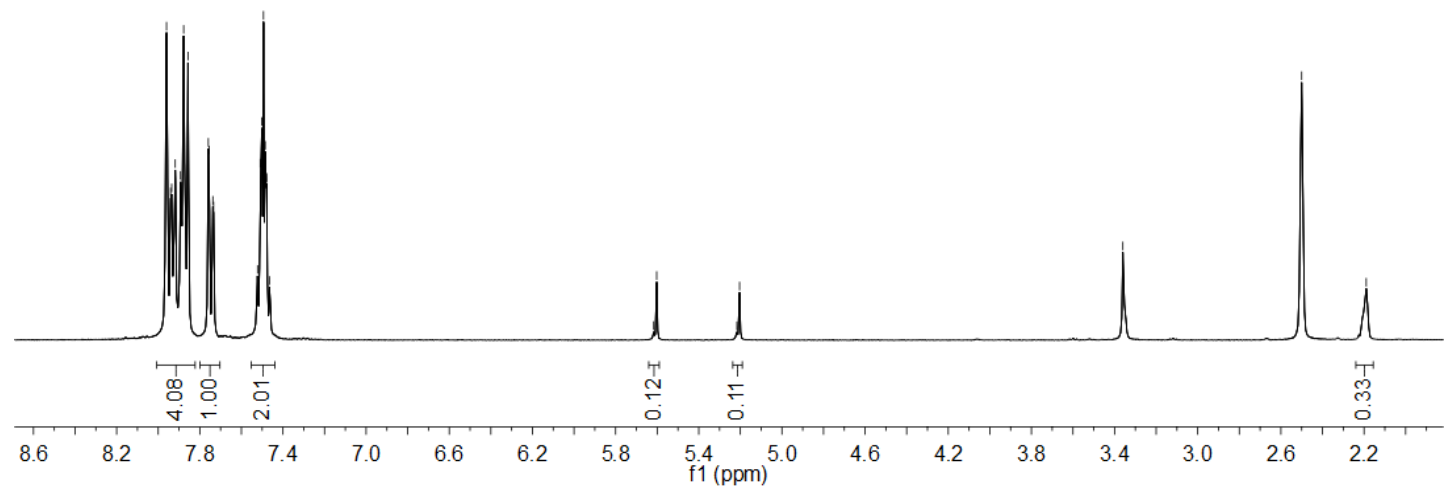

\subsubsection{Deuterium-labeling studies with $1 \mathrm{q}-\mathrm{D}_{5}$ and $2 \mathrm{a}$}
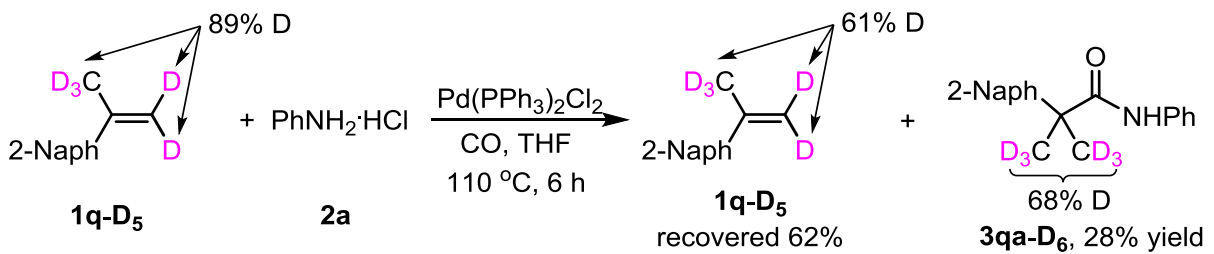
A mixture of 1q-D5 $(0.2 \mathrm{mmol}, 1.0$ equiv, $89 \%$ D), $\mathbf{2 a}(0.2 \mathrm{mmol}, 1.0$ equiv), $\mathrm{Pd}\left(\mathrm{PPh}_{3}\right)_{2} \mathrm{Cl}_{2}(0.006 \mathrm{mmol}, 3 \mathrm{~mol} \%)$, and anhydrous THF $(1.2 \mathrm{~mL})$ were added into a glass tube which was placed in an autoclave. The autoclave was evacuated and backfilled with $\mathrm{CO}$ for three times in a well-ventilated fume hood, and then pressurized to $45 \mathrm{~atm}$ of $\mathrm{CO}$. The reaction mixture in autoclave was stirred at $110{ }^{\circ} \mathrm{C}$ for 6 hours. After that, the autoclave was removed from the oil bath and cooled to room temperature prior to the release of excess carbon monoxide. Then the corresponding reaction mixture was purified by flash column chromatography on a silica gel column (petroleum ether/ethyl acetate $=10 / 1$ ) to give the product $\mathbf{3 q a} \mathbf{a}-\mathbf{D}_{\mathbf{6}}$ in $28 \%$ yield (68 atom \% D in $\mathbf{3 q a}-\mathbf{D}_{\mathbf{6}}$ was determined by ${ }^{1} \mathrm{H} \mathrm{NMR}$ ), and the $\mathbf{1 q - \mathbf { D } _ { 5 }}$ was recovered in $62 \%$ yield (61 atom \% D in $\mathbf{1 q - D _ { 5 }}$ was determined by ${ }^{1} \mathrm{H}$ NMR).

${ }^{1} \mathrm{H}$ NMR (400 MHz, $d^{6}$-DMSO) of $\mathbf{1 q - \mathbf { D } _ { 5 }}$

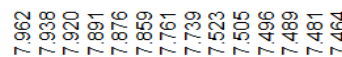
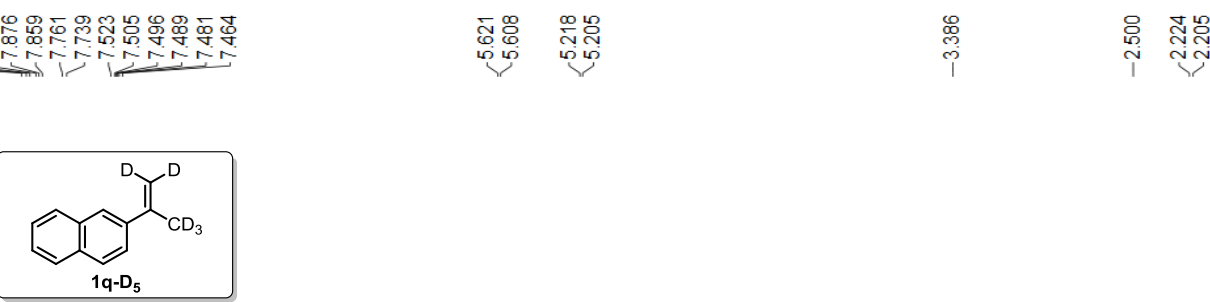

$D \%=1-\frac{\frac{0.40+0.38+1.18}{1+1+3}}{\frac{4.08+1.00+2.04}{4+1+2}}=1-\frac{0.392}{1.017}=61 \%$

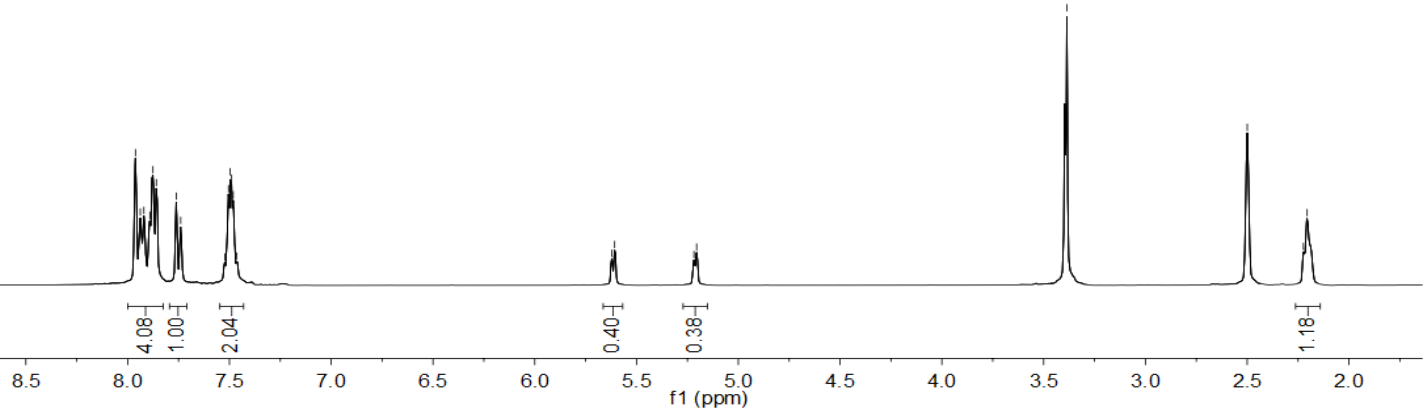


${ }^{1} \mathrm{H}$ NMR (400 MHz, $d^{6}$-DMSO) of compound 3qa-D 6

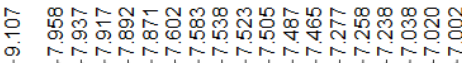

总

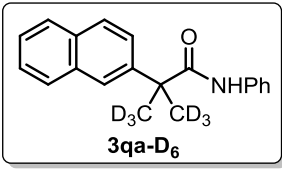

D $\%=1-\frac{\frac{1.92}{6}}{\frac{1.01+4.19+4.79+2.00+0.95}{1+4+5+2+1}}=1-\frac{0.32}{0.995}=68 \%$.

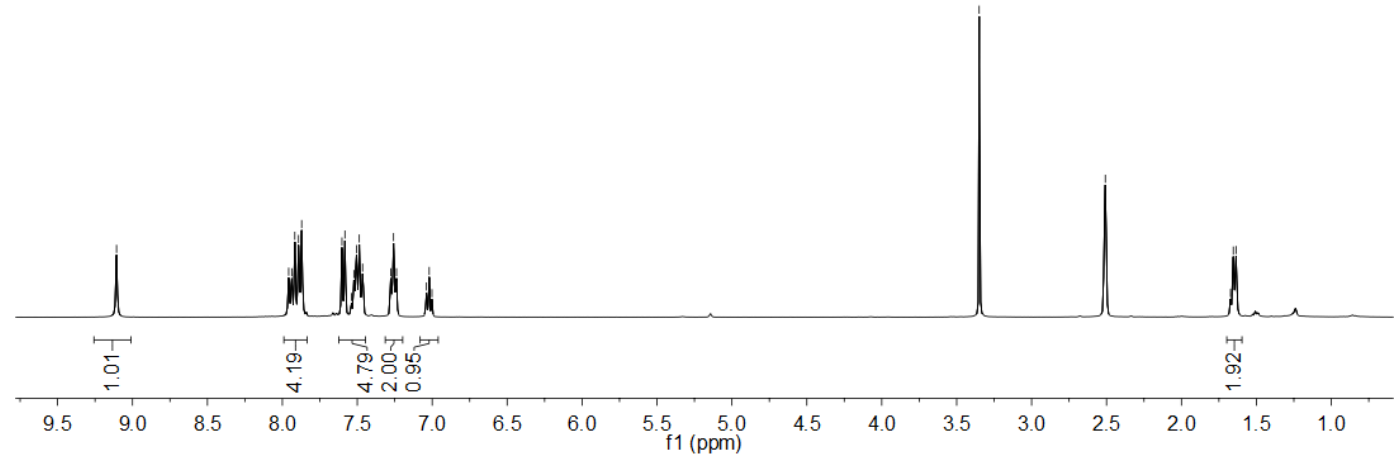

\subsection{Control experiments}

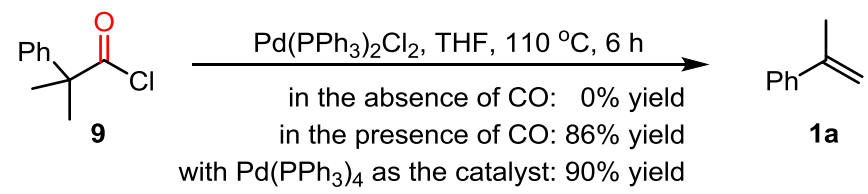

The 2-methyl-2-phenylpropanoyl chloride 9 ( $0.2 \mathrm{mmol}, 1.0$ equiv), $\mathrm{Pd}\left(\mathrm{PPh}_{3}\right)_{2} \mathrm{Cl}_{2}$ (0.006 mmol, $3 \mathrm{~mol} \%)$, and anhydrous THF $(1.2 \mathrm{~mL})$ were added into a glass tube which was placed in an autoclave. The autoclave was evacuated and backfilled with Ar for three times in a well-ventilated fume hood, and then pressurized to $10 \mathrm{~atm}$ of Ar. The reaction mixture in autoclave was stirred at $110^{\circ} \mathrm{C}$ for 6 hours under Ar. After completion, the autoclave was removed from the oil bath and cooled to room temperature prior to the release of Ar. The alkene 1a was not observed.

When the autoclave was evacuated and backfilled with $\mathrm{CO}$ for three times in a well-ventilated fume hood, and then pressurized to $45 \mathrm{~atm}$ of $\mathrm{CO}$. The alkene 1a was obtained in $86 \%$ yield. 
When $\mathrm{Pd}\left(\mathrm{PPh}_{3}\right)_{4}$ was used as catalyst, the autoclave was evacuated and backfilled with Ar for three times, and then pressurized to $10 \mathrm{~atm}$ of Ar. After reaction, the 1a was obtained in $90 \%$ yield.

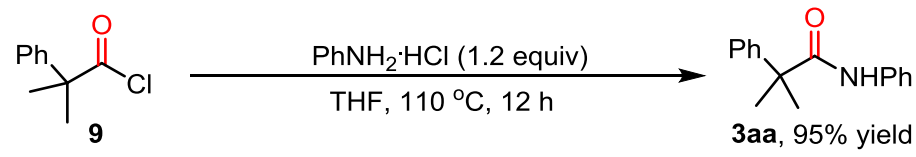

A mixture of 2-methyl-2-phenylpropanoyl chloride 9 (0.20 mmol, 1.0 equiv), aniline hydrochloride salt $\mathbf{2 a}(0.24 \mathrm{mmol}, 1.2$ equiv) and anhydrous THF (1.2 mL) were added to a $10 \mathrm{ml}$ round bottomed flask under argon, the mixture was stirred at $110^{\circ} \mathrm{C}$ for 12 hours. Then the corresponding reaction mixture was purified by flash column chromatography on a silica gel column (petroleum ether/ethyl acetate $=10 / 1$ ) to give the 3aa in $95 \%$ yield.

\subsection{Hammett plot analysis}

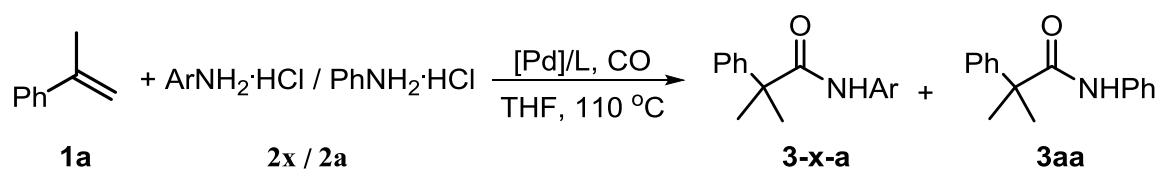

A mixture of $\alpha$-methyl styrene 1a $\left(0.2 \mathrm{mmol}, 1.0\right.$ equiv), $\mathrm{ArNH}_{2} \cdot \mathrm{HCl} \mathbf{2 x}(0.2$ mmol, 1.0 equiv), $\mathrm{PhNH}_{2} . \mathrm{HCl} 2 \mathbf{a}\left(0.2 \mathrm{mmol}, 1.0\right.$ equiv), $\mathrm{PdCl}_{2}\left(\mathrm{PPh}_{3}\right)_{2}(0.006 \mathrm{mmol}, 3$ mol\%), and THF (1.2 mL) were added into a glass tube which was placed in an autoclave. The autoclave was evacuated and backfilled with $\mathrm{CO}$ for three times in a well-ventilated fume hood, and then pressurized to $45 \mathrm{~atm}$ of $\mathrm{CO}$. The reaction mixture in autoclave was stirred at $110^{\circ} \mathrm{C}$ for 24 hours. After that, the autoclave was removed from the oil bath and cooled to room temperature prior to the release of excess carbon monoxide. Then the corresponding reaction mixture was purified by flash column chromatography on a silica gel column (petroleum ether/ethyl acetate $=$ 10/1) to give the mixture products. The ratio of different products was determined by the ${ }^{1} \mathrm{H}$ NMR analysis. See following table. 


\begin{tabular}{|c|c|c|c|c|c|}
\hline $\mathrm{ArNH}_{2} \cdot \mathrm{HCl}$ & $\sigma$ & Conv. of $1 \mathbf{a}$ & Ratio of $Y_{3-\mathbf{x}-\mathbf{a}}: Y_{3 a a}$ & $\mathrm{k}_{\mathrm{x}} / \mathrm{k}_{\mathrm{H}}$ & $\log \left(\mathrm{k}_{\mathrm{x}} / \mathrm{k}_{\mathrm{H}}\right)$ \\
\hline$p$-OMe & -0.268 & $86 \%$ & $25 \%: 61 \%$ & 0.41 & -0.387 \\
\hline$p$-Me & -0.170 & $82 \%$ & $28 \%: 54 \%$ & 0.51 & -0.292 \\
\hline$p-\mathrm{Cl}$ & 0.227 & $77 \%$ & $47 \%: 30 \%$ & 1.57 & 0.196 \\
\hline$p-\mathrm{Br}$ & 0.232 & $83 \%$ & $51 \%: 32 \%$ & 1.59 & 0.201 \\
\hline
\end{tabular}

Figure S1 Hammett plot. The conversion of 1a was calculated based on mixture products, the ratio of $\mathrm{Y}_{3-\mathrm{x}-\mathrm{a}} / \mathrm{Y}_{\mathbf{3 a a}}$ was determined by ${ }^{1} \mathrm{H}$ NMR.

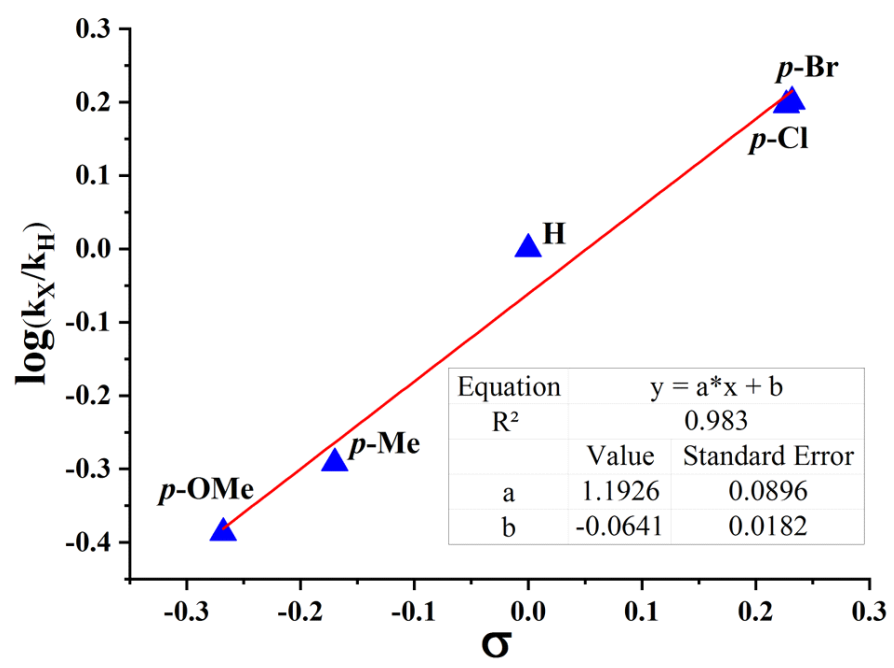

Figure S2. Hammett plot for the reaction using para-substituted anilines hydrochloride. Logarithm of the ratio of rate constant $\left(\log \left(\mathrm{k}_{\mathrm{X}} / \mathrm{k}_{\mathrm{H}}\right)\right.$ versus $\sigma \mathrm{p}$ for the hydroaminocarbonylation reaction of $p$-substituted anilines hydrochloride with $\alpha$-methyl styrene. 


\section{Characterization data of products}

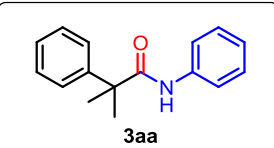

\section{2-methyl-N,2-diphenylpropanamide (3aa)}

Yield $=96 \%, \mathrm{~b} / \mathrm{l}>99: 1,{ }^{1} \mathrm{H}$ NMR $\left(400 \mathrm{MHz}, \mathrm{CDCl}_{3}\right) \delta$ 7.45-7.37 (m, 4H), 7.36-7.29 (m, 3H), 7.26-7.22 (m, 2H), 7.06-7.02 (m 1H), $6.81(\mathrm{~s}, 1 \mathrm{H}), 1.66(\mathrm{~s}, 6 \mathrm{H})$; ${ }^{13} \mathrm{C}$ NMR $\left(101 \mathrm{MHz}, \mathrm{CDCl}_{3}\right) \delta 175.6,144.7,138.1,128.9,129.0,127.5,126.6,124.2$, 119.8, 48.1, 27.1. HRMS calcd. (ESI) $\mathrm{m} / \mathrm{z}$ for $\mathrm{C}_{16} \mathrm{H}_{17} \mathrm{NNaO}[\mathrm{M}+\mathrm{Na}]^{+}$: 262.1202, found: 262.1202 .

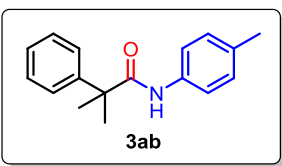

\section{2-methyl-2-phenyl- $N$-(p-tolyl)propanamide (3ab)}

Yield $=85 \%, \mathrm{~b} / \mathrm{l}=98: 2 ;{ }^{1} \mathrm{H} \mathrm{NMR}\left(400 \mathrm{MHz}, \mathrm{CDCl}_{3}\right) \delta 7.45-7.37(\mathrm{~m}, 4 \mathrm{H}), 7.30$ (t, $J=7.0 \mathrm{~Hz}, 1 \mathrm{H}), 7.23(\mathrm{~d}, J=8.4 \mathrm{~Hz}, 2 \mathrm{H}), 7.05(\mathrm{~d}, J=8.4 \mathrm{~Hz}, 2 \mathrm{H}), 6.77(\mathrm{~s}, 1 \mathrm{H})$, $2.26(\mathrm{~s}, 3 \mathrm{H}), 1.65(\mathrm{~s}, 6 \mathrm{H}) ;{ }^{13} \mathrm{C} \mathrm{NMR}\left(101 \mathrm{MHz}, \mathrm{CDCl}_{3}\right) \delta 175.5,144.8,135.5,133.8$, 129.4, 129.0, 127.4, 126.5, 119.8, 48.0, 27.1, 20.9. HRMS calcd (ESI) m/z for $\mathrm{C}_{17} \mathrm{H}_{19} \mathrm{NNaO}[\mathrm{M}+\mathrm{Na}]^{+}:$273.1358, found: 276.1359 .

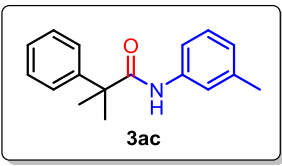

\section{2-methyl-2-phenyl- $N$-(m-tolyl)propanamide (3ac)}

Yield $=92 \%, \mathrm{~b} / \mathrm{l}=98: 2 ;{ }^{1} \mathrm{H}$ NMR $\left(400 \mathrm{MHz}, \mathrm{CDCl}_{3}\right) \delta$ 7.45-7.37 (m, 4H), 7.33-7.28 (m, 1H), $7.23(\mathrm{~d}, J=1.6 \mathrm{~Hz}, 1 \mathrm{H}), 7.13-7.11(\mathrm{~m}, 2 \mathrm{H}), 6.87-6.85(\mathrm{~m}, 1 \mathrm{H})$, $6.78(\mathrm{~s}, 1 \mathrm{H}), 2.28(\mathrm{~s}, 3 \mathrm{H}), 1.65(\mathrm{~s}, 6 \mathrm{H}) ;{ }^{13} \mathrm{C} \mathrm{NMR}\left(101 \mathrm{MHz}, \mathrm{CDCl}_{3}\right) \delta$ 175.6, 144.7, 138.9, 138.0, 129.1, 128.8, 127.4, 126.5, 125.0, 120.4, 116.8, 48.1, 27.1, 21.5. HRMS calcd. (ESI) $\mathrm{m} / \mathrm{z}$ for $\mathrm{C}_{17} \mathrm{H}_{19} \mathrm{NNaO}[\mathrm{M}+\mathrm{Na}]^{+}$: 273.1358, found: 273.1358 . 


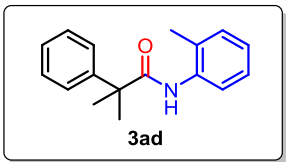

2-methyl-2-phenyl- $N$-(o-tolyl)propanamide (3ad)

Yield $=76 \%, \mathrm{~b} / \mathrm{l}=97: 3,{ }^{1} \mathrm{H} \mathrm{NMR}\left(400 \mathrm{MHz}, \mathrm{CDCl}_{3}\right) \delta 7.87(\mathrm{~d}, J=8.0 \mathrm{~Hz}, 1 \mathrm{H})$, 7.51-7.48 (m, 2H), 7.44-7.40 (m, 2H), 7.34-7.31 (m, 1H), $7.17(\mathrm{t}, J=7.6 \mathrm{~Hz}, 1 \mathrm{H})$, $7.05(\mathrm{~d}, J=7.2 \mathrm{~Hz}, 1 \mathrm{H}), 6.99$ (t, $J=7.6 \mathrm{~Hz}, 1 \mathrm{H}), 6.69$ (s, 1H), 1.78 (s, 3H), 1.70 (s, $6 \mathrm{H}) ;{ }^{13} \mathrm{C} \mathrm{NMR}\left(101 \mathrm{MHz}, \mathrm{CDCl}_{3}\right) \delta 175.7,144.8,136.0,130.3,129.1,128.0,127.6$, 126.9, 126.8, 124.7, 121.9, 48.2, 27.0, 17.1. HRMS calcd (ESI) $\mathrm{m} / \mathrm{z}$ for $\mathrm{C}_{17} \mathrm{H}_{19} \mathrm{NO}$ : $[\mathrm{M}+\mathrm{Na}]^{+} 273.1358$, found: 276.1353 .

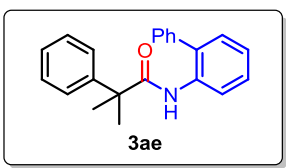

\section{$N$-([1,1'-biphenyl]-2-yl)-2-methyl-2-phenylpropanamide (3ae)}

Yield $=91 \%$, b/l > 99:1, ${ }^{1} \mathrm{H}$ NMR $\left(400 \mathrm{MHz}, \mathrm{CDCl}_{3}\right) \delta 8.40(\mathrm{~d}, J=8.4 \mathrm{~Hz}, 1 \mathrm{H})$, 7.36-7.26 (m, 2H), 7.25-7.17 (m, 7H), 7.13-7.07 (m, 2H),7.01 (s, 1H), 6.97-6.94(m, 2H) $1.51(\mathrm{~s}, 6 \mathrm{H}) ;{ }^{13} \mathrm{C} \mathrm{NMR}\left(101 \mathrm{MHz}, \mathrm{CDCl}_{3}\right) \delta 175.4,144.0,137.7,135.3,131.9$, 130.0 129.0, 128.9, 128.8, 128.5, 127.6, 127.2, 126.2, 123.8, 120.3, 48.1, 26.8. HRMS calcd. (ESI) $\mathrm{m} / \mathrm{z}$ for $\mathrm{C}_{22} \mathrm{H}_{21} \mathrm{NNaO}[\mathrm{M}+\mathrm{Na}]^{+}: 338.1515$, found: 338.1515 .

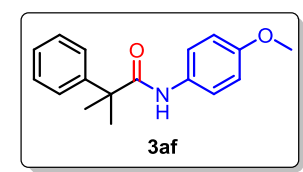

\section{$\mathrm{N}$-(4-methoxyphenyl)-2-methyl-2-phenylpropanamide (3af)}

Yield $=67 \%, \mathrm{~b} / \mathrm{l}>99: 1 ;{ }^{1} \mathrm{H}$ NMR $\left(400 \mathrm{MHz}, \mathrm{CDCl}_{3}\right) \delta$ 7.50-7.37 (m, 4H), 7.33-7.29 (m, 1H), 7.28-7.14 (m, 2H), 6.83-6.76 (m, 2H), $6.71(\mathrm{~s}, 1 \mathrm{H}), 3.76(\mathrm{~s}, 3 \mathrm{H})$, $1.66(\mathrm{~s}, 6 \mathrm{H}) ;{ }^{13} \mathrm{C} \mathrm{NMR}\left(101 \mathrm{MHz}, \mathrm{CDCl}_{3}\right) \delta 175.6,156.4,144.9,131.2,129.1,127.5$, 126.6, 121.7, 114.1, 55.6, 48.0, 27.2. HRMS calcd. (ESI) $\mathrm{m} / \mathrm{z}$ for $\mathrm{C}_{17} \mathrm{H}_{19} \mathrm{NNaO}_{2}$ $[\mathrm{M}+\mathrm{Na}]^{+}:$292.1308, found: 292.1311 . 


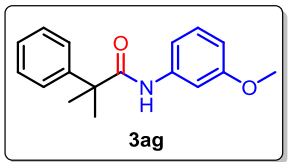

$N$-(3-methoxyphenyl)-2-methyl-2-phenylpropanamide (3ag)

Yield $=83 \%, \mathrm{~b} / \mathrm{l}=96: 4,{ }^{1} \mathrm{H} \mathrm{NMR}\left(400 \mathrm{MHz}, \mathrm{CDCl}_{3}\right) \delta 7.45-7.37(\mathrm{~m}, 4 \mathrm{H}), 7.31$ $(\mathrm{t}, J=7.0 \mathrm{~Hz}, 1 \mathrm{H}), 7.24(\mathrm{t}, J=2.2 \mathrm{~Hz}, 1 \mathrm{H}), 7.11(\mathrm{t}, J=8.2 \mathrm{~Hz}, 1 \mathrm{H}), 6.83(\mathrm{~s}, 1 \mathrm{H})$, 6.74-6.71 (m, 1H), 6.62-6.59 (m, 1H), $3.76(\mathrm{~s}, 3 \mathrm{H}), 1.66(\mathrm{~s}, 6 \mathrm{H}) ;{ }^{13} \mathrm{C}$ NMR $(101 \mathrm{MHz}$, $\left.\mathrm{CDCl}_{3}\right) \delta 175.7,160.2,144.6,139.3,129.5,129.1,127.5,126.5,111.7,110.3,105.2$, 55.3, 48.2, 27.1. HRMS calcd. (ESI) $\mathrm{m} / \mathrm{z}$ for $\mathrm{C}_{17} \mathrm{H}_{19} \mathrm{NNaO}_{2}[\mathrm{M}+\mathrm{Na}]^{+}$: 292.1308, found: 292.1312 .

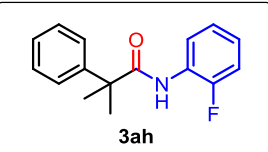

\section{$N$-(2-fluorophenyl)-2-methyl-2-phenylpropanamide (3ah)}

Yield $=90 \%, \mathrm{~b} / \mathrm{l}=90: 10,{ }^{1} \mathrm{H}$ NMR $\left(400 \mathrm{MHz}, \mathrm{CDCl}_{3}\right) \delta 8.30-8.25(\mathrm{~m}, 1 \mathrm{H})$, 7.47-7.44 (m, 2H), 7.42-7.38 (m, 2H), 7.32-7.29 (m, 1H), 7.13-7.06 (m, 2H), 6.99-6.93 (m, 2H), $1.68(\mathrm{~s}, 6 \mathrm{H}) ;{ }^{13} \mathrm{C}$ NMR (101 MHz, $\left.\mathrm{CDCl}_{3}\right) \delta 175.8,153.7,151.3$, 144.2, 129.1, 127.6, 126.5, $124.6(\mathrm{~d}, J=3.3 \mathrm{~Hz}), 124.2(\mathrm{~d}, J=7.6 \mathrm{~Hz}), 121.6,114.7$ $(\mathrm{d}, J=19.1 \mathrm{~Hz}), 48.3,27.0 ;{ }^{19} \mathrm{~F}$ NMR $\left(376 \mathrm{MHz}, \mathrm{CDCl}_{3}\right) \delta$-114.9. HRMS calcd. (ESI) $\mathrm{m} / \mathrm{z}$ for $\mathrm{C}_{16} \mathrm{H}_{16} \mathrm{FNNaO}[\mathrm{M}+\mathrm{Na}]^{+}: 280.1108$, found: 280.1119 .

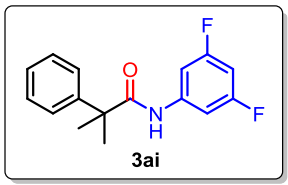

\section{$N$-(3,5-difluorophenyl)-2-methyl-2-phenylpropanamide (3ai)}

Yield $=88 \%, \mathrm{~b} / \mathrm{l}=95: 5,{ }^{1} \mathrm{H}$ NMR $\left(400 \mathrm{MHz}, \mathrm{CDCl}_{3}\right) \delta$ 7.41-7.29 (m, 5H), 7.00-6.95 (m, 3H), 6.49-6.44 (m, 1H), $1.64(\mathrm{~s}, 6 \mathrm{H}) ;{ }^{13} \mathrm{C}$ NMR (101 MHz, $\left.\mathrm{CDCl}_{3}\right) \delta$ $175.9,163.1(\mathrm{dd}, J=244.7,14.6 \mathrm{~Hz}), 144.0,140.2(\mathrm{t}, J=13.3 \mathrm{~Hz}), 129.2,127.7$, 126.4, 102.7 (dd, $J=20.6,8.4 \mathrm{~Hz}), 99.3$ (t, $J=25.4 \mathrm{~Hz}), 48.3,26.9 ;{ }^{19} \mathrm{~F}$ NMR $(376$ $\mathrm{MHz}, \mathrm{CDCl}_{3}$ ) $\delta$-109.0. HRMS calcd (ESI) $\mathrm{m} / \mathrm{z}$ for $\mathrm{C}_{16} \mathrm{H}_{15} \mathrm{~F}_{2} \mathrm{NNaO}[\mathrm{M}+\mathrm{Na}]^{+}$: 298.1013, found: 298.0998 . 


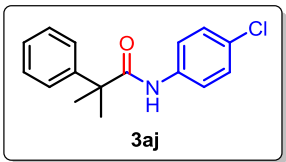

$N$-(4-chlorophenyl)-2-methyl-2-phenylpropanamide (3aj)

Yield $=94 \%, \mathrm{~b} / \mathrm{l}>99: 1 ;{ }^{1} \mathrm{H}$ NMR $\left(400 \mathrm{MHz}, \mathrm{CDCl}_{3}\right) \delta$ 7.44-7.38 (m, 4H), 7.34-7.27 (m, 3H), 7.21-7.17 (m, 2H), 6.83 (s, 1H), 1.65 (s, 6H); ${ }^{13} \mathrm{C}$ NMR (101 MHz, $\left.\mathrm{CDCl}_{3}\right) \delta 175.0,143.3,137.9,133.4,129.2,129.0,128.0,124.4,119.9,47.8,27.1$. HRMS calcd. (ESI) m/z for $\mathrm{C}_{16} \mathrm{H}_{16} \mathrm{ClNNaO}[\mathrm{M}+\mathrm{Na}]^{+}$: 296.0812, found: 296.0798.

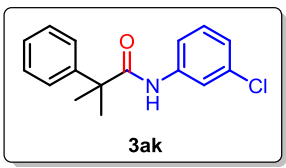

\section{$N$-(3-chlorophenyl)-2-methyl-2-phenylpropanamide (3ak)}

Yield $=93 \%, \mathrm{~b} / \mathrm{l}=98: 2 ;{ }^{1} \mathrm{H} \mathrm{NMR}\left(400 \mathrm{MHz}, \mathrm{CDCl}_{3}\right) \delta 7.49(\mathrm{~d}, J=2.0 \mathrm{~Hz}, 1 \mathrm{H})$, 7.43-7.38 (m, 4H), 7.36-7.29 (m, 1H), 7.18-7.11 (m, 2H), 7.02-6.99 (m, 1H), $6.87(\mathrm{~s}$, 1H), $1.65(\mathrm{~s}, 6 \mathrm{H}) ;{ }^{13} \mathrm{C} \mathrm{NMR}\left(101 \mathrm{MHz}, \mathrm{CDCl}_{3}\right) \delta 175.8,144.3,139.2,134.6,129.9$, 129.2, 127.6, 126.5, 124.2, 119.9, 117.7, 48.2, 27.0. HRMS calcd. (ESI) m/z for $\mathrm{C}_{16} \mathrm{H}_{16} \mathrm{ClNNaO}[\mathrm{M}+\mathrm{Na}]^{+}:$296.0812, found: 296.0810 .

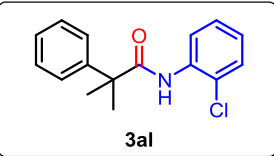

\section{$N$-(2-chlorophenyl)-2-methyl-2-phenylpropanamide (3al)}

Yield $=95 \%, \mathrm{~b} / \mathrm{l}=96: 4 ;{ }^{1} \mathrm{H} \mathrm{NMR}\left(400 \mathrm{MHz}, \mathrm{CDCl}_{3}\right) \delta 8.35(\mathrm{dd}, J=9.6,1.4 \mathrm{~Hz}$, 1H), 7.49-7.47 (m, 3H), 7.43-7.39 (m, 2H), 7.36-7.29 (m, 1H), 7.24-7.20 (m, 2H), 6.97-6.93 (m, 1H), $1.70(\mathrm{~s}, 6 \mathrm{H}) ;{ }^{13} \mathrm{C} \mathrm{NMR}\left(101 \mathrm{MHz}, \mathrm{CDCl}_{3}\right) \delta 175.8,144.1,134.9$, 129.1, 128.9, 127.7, 127.6, 126.6, 124.4, 122.9, 121.2, 48.4, 26.9. HRMS calcd. (ESI) $\mathrm{m} / \mathrm{z}$ for $\mathrm{C}_{16} \mathrm{H}_{16} \mathrm{ClNNaO}[\mathrm{M}+\mathrm{Na}]^{+}: 296.0812$, found: 296.0822 . 


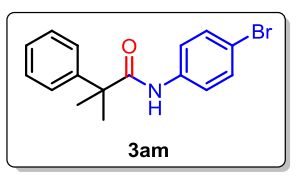

$N$-(4-chlorophenyl)-2-methyl-2-phenylpropanamide (3am)

Yield $=93 \%, \mathrm{~b} / \mathrm{l}>99: 1,{ }^{1} \mathrm{H}$ NMR $\left(400 \mathrm{MHz}, \mathrm{CDCl}_{3}\right) \delta$ 7.44-7.38 (m, 4H), 7.36-7.29 (m, 3H), 7.26-7.22 (m, 2H), $6.82(\mathrm{~s}, 1 \mathrm{H}), 1.65$ (s, 6H); ${ }^{13} \mathrm{C}$ NMR (101 MHz, $\left.\mathrm{CDCl}_{3}\right) \delta 175.7,144.4,137.1,131.9,129.2,127.6,126.5,121.4,116.8,48.2,27.1$. HRMS calcd. (ESI) m/z for $\mathrm{C}_{16} \mathrm{H}_{16} \mathrm{BrNNaO}[\mathrm{M}+\mathrm{Na}]^{+}: 340.0307$, found: 340.0307 .

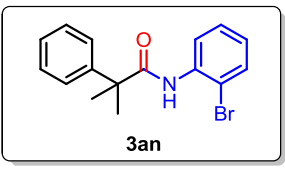

$N$-(2-bromophenyl)-2-methyl-2-phenylpropanamide (3an)

Yield $=64 \%, \mathrm{~b} / \mathrm{l}>99: 1,{ }^{1} \mathrm{H} \mathrm{NMR}\left(400 \mathrm{MHz}, \mathrm{CDCl}_{3}\right) \delta 8.34(\mathrm{dd}, J=8.4,1.6 \mathrm{~Hz}$, 1H), 7.50-7.47 (m, 3H), 7.44-7.39 (m, 3H), 7.34-7.25 (m, 2H), 6.92-6.88 (m, 1H), $1.71(\mathrm{~s}, 6 \mathrm{H}) ;{ }^{13} \mathrm{C} \mathrm{NMR}\left(101 \mathrm{MHz}, \mathrm{CDCl}_{3}\right) \delta 175.9,144.1,136.0,132.2,129.2,128.3$, 127.6, 126.7, 124.9, 121.4, 113.4, 48.4, 26.9. HRMS calcd. (ESI) $\mathrm{m} / \mathrm{z}$ for $\mathrm{C}_{16} \mathrm{H}_{16} \mathrm{BrNNaO}[\mathrm{M}+\mathrm{Na}]^{+}:$340.0307, found: 340.0298 .

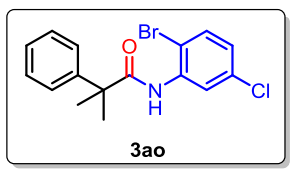

\section{$N$-(2-bromo-4-chlorophenyl)-2-methyl-2-phenylpropanamide (3ao)}

Yield $=72 \%$, b/l > 99:1, ${ }^{1} \mathrm{H}$ NMR $\left(400 \mathrm{MHz}, \mathrm{CDCl}_{3}\right) \delta 8.47(\mathrm{~d}, J=2.4 \mathrm{~Hz}, 1 \mathrm{H})$, 7.49-7.40 (m, 5H), 7.35-7.30 (m, 2H), $6.88(\mathrm{dd}, J=8.8,2.4 \mathrm{~Hz}, 1 \mathrm{H}), 1.70(\mathrm{~s}, 6 \mathrm{H}) ;{ }^{13} \mathrm{C}$ NMR $\left(101 \mathrm{MHz}, \mathrm{CDCl}_{3}\right) \delta 176.0,143.7,136.8,134.3,132.7,129.3,127.8,126.7$, 124.8, 121.1, 110.8, 48.5, 26.8. HRMS calcd (ESI) $\mathrm{m} / \mathrm{z}$ for $\mathrm{C}_{16} \mathrm{H}_{15} \mathrm{BrClNNaO}$ $[\mathrm{M}+\mathrm{Na}]^{+}:$373.9917, found: 373.9916 . 


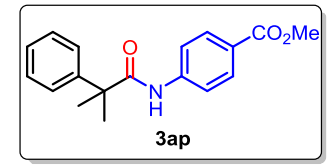

methyl 4-(2-methyl-2-phenylpropanamido)benzoate (3ap)

Yield $=84 \%, \mathrm{~b} / \mathrm{l}>99: 1,{ }^{1} \mathrm{H}$ NMR $\left(400 \mathrm{MHz}, \mathrm{CDCl}_{3}\right) \delta 7.90(\mathrm{~d}, J=8.8 \mathrm{~Hz}, 2 \mathrm{H})$, 7.46-7.36 (m, 6H), 7.32-7.27 (m, 1H), $7.18(\mathrm{~s}, 1 \mathrm{H}), 3.82(\mathrm{~s}, 3 \mathrm{H}), 1.66(\mathrm{~s}, 6 \mathrm{H}) ;{ }^{13} \mathrm{C}$ NMR $\left(101 \mathrm{MHz}, \mathrm{CDCl}_{3}\right) \delta 175.9,166.6,144.2,142.3,130.7,129.1,127.5,126.4$, 125.3, 118.8, 51.9, 48.2, 26.9. HRMS calcd. (ESI) $\mathrm{m} / \mathrm{z}$ for $\mathrm{C}_{18} \mathrm{H}_{19} \mathrm{NNaO}_{3}[\mathrm{M}+\mathrm{Na}]^{+}$: 320.1257, found: 320.1266 .

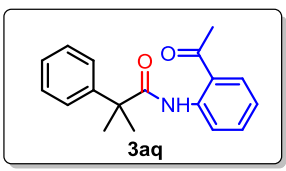

$N$-(2-acetylphenyl)-2-methyl-2-phenylpropanamide (3aq)

Yield $=91 \%, \mathrm{~b} / \mathrm{l}=93: 7,{ }^{1} \mathrm{H}$ NMR $\left(400 \mathrm{MHz}, \mathrm{CDCl}_{3}\right) \delta 11.56(\mathrm{~s}, 1 \mathrm{H}), 8.79(\mathrm{dd}, J$ $=8.4,0.8 \mathrm{~Hz}, 1 \mathrm{H}), 7.81(\mathrm{dd}, J=8.0,1.2 \mathrm{~Hz}, 1 \mathrm{H}), 7.54-7.46(\mathrm{~m}, 3 \mathrm{H}), 7.39-7.35(\mathrm{~m}$, 2H), 7.29-7.25 (m, 1H), 7.07-7.03 (m, 1H), $2.54(\mathrm{~s}, 3 \mathrm{H}), 1.70(\mathrm{~s}, 6 \mathrm{H}) ;{ }^{13} \mathrm{C}$ NMR $(101$ $\left.\mathrm{MHz}, \mathrm{CDCl}_{3}\right) \delta 202.4,176.8,144.7,141.3,135.0,131.6,128.7,127.0,126.2,122.1$, 121.8, 120.5, 48.4, 28.5, 26.8. HRMS calcd (ESI) $\mathrm{m} / \mathrm{z}$ for $\mathrm{C}_{18} \mathrm{H}_{19} \mathrm{NNaO}_{2}[\mathrm{M}+\mathrm{Na}]^{+}$: 304.1308, found: 304.1318 .

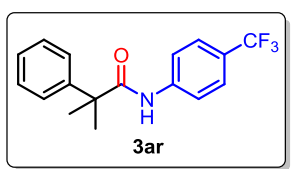

\section{2-methyl-2-phenyl- $N$-(4-(trifluoromethyl)phenyl)propanamide (3ar)}

Yield $=84 \%, \mathrm{~b} / \mathrm{l}>99: 1,{ }^{1} \mathrm{H}$ NMR $\left(400 \mathrm{MHz}, \mathrm{CDCl}_{3}\right) \delta 7.50-7.39(\mathrm{~m}, 8 \mathrm{H})$ 7.35-7.30 (m, 1H), $7.02(\mathrm{~s}, 1 \mathrm{H}), 1.66(\mathrm{~s}, 6 \mathrm{H}) ;{ }^{13} \mathrm{C} \mathrm{NMR}\left(101 \mathrm{MHz}, \mathrm{CDCl}_{3}\right) \delta 176.0$, $144.2,141.1,129.2,127.7,126.5,126.2(\mathrm{q}, J=7.5 \mathrm{~Hz}, 3.7 \mathrm{~Hz}), 125.6(\mathrm{~d}, J=25.7 \mathrm{~Hz})$, 122.8, 119.3, 48.3, 27.0. ${ }^{19} \mathrm{~F}$ NMR (376 MHz, $\left.\mathrm{CDCl}_{3}\right) \delta$-32.6. HRMS calcd. (ESI) $\mathrm{m} / \mathrm{z}$ for $\mathrm{C}_{17} \mathrm{H}_{16} \mathrm{~F}_{3} \mathrm{NNaO}[\mathrm{M}+\mathrm{Na}]^{+}: 330.1076$, found: 330.1080 . 


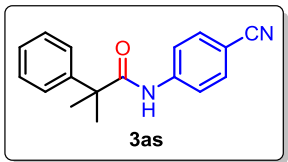

\section{$N$-(4-cyanophenyl)-2-methyl-2-phenylpropanamide (3as)}

Yield $=58 \%, \mathrm{~b} / \mathrm{l}=97: 3,{ }^{1} \mathrm{H}$ NMR $\left(400 \mathrm{MHz}, \mathrm{CDCl}_{3}\right) \delta$ 7.54-7.48 (m, 4H), 7.42 $(\mathrm{d}, J=4.4 \mathrm{~Hz}, 4 \mathrm{H}), 7.37-7.31(\mathrm{~m}, 1 \mathrm{H}), 7.05(\mathrm{~s}, 1 \mathrm{H}), 1.67(\mathrm{~s}, 6 \mathrm{H}) ;{ }^{13} \mathrm{C}$ NMR $(101$ $\left.\mathrm{MHz}, \mathrm{CDCl}_{3}\right) \delta 176.1,143.9,142.1,133.1,129.2,127.7,126.4,119.5,118.9,106.8$, 48.3, 26.9. HRMS calcd. (ESI) $\mathrm{m} / \mathrm{z}$ for $\mathrm{C}_{17} \mathrm{H}_{16} \mathrm{~N}_{2} \mathrm{NaO}[\mathrm{M}+\mathrm{Na}]^{+}: 287.1154$, found: 287.1165.

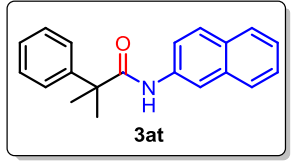

2-methyl- $N$-(naphthalen-2-yl)-2-phenylpropanamide (3at)

Yield $=78 \%, \mathrm{~b} / \mathrm{l}=90: 10,{ }^{1} \mathrm{H} \mathrm{NMR}\left(400 \mathrm{MHz}, \mathrm{CDCl}_{3}\right) \delta 8.10(\mathrm{~s}, 1 \mathrm{H}), 7.73-7.67$ $(\mathrm{m}, 3 \mathrm{H}), 7.46(\mathrm{~d}, J=7.6 \mathrm{~Hz}, 2 \mathrm{H}), 7.40-7.30(\mathrm{~m}, 5 \mathrm{H}), 7.21(\mathrm{dd}, J=8.8,2.0 \mathrm{~Hz}, 1 \mathrm{H})$, $6.99(\mathrm{~s}, 1 \mathrm{H}), 1.69(\mathrm{~s}, 6 \mathrm{H}) ;{ }^{13} \mathrm{C} \mathrm{NMR}\left(101 \mathrm{MHz}, \mathrm{CDCl}_{3}\right) \delta 175.9,144.6,135.5,133.9$, 130.6, 129.1, 128.6, 127.7, 127.6, 127.5, 126.6, 126.5, 125.0, 119.9, 116.4, 48.2, 27.2. HRMS calcd. (ESI) m/z for $\mathrm{C}_{20} \mathrm{H}_{19} \mathrm{NNaO}[\mathrm{M}+\mathrm{Na}]^{+}: 312.1358$, found: 312.1359 .

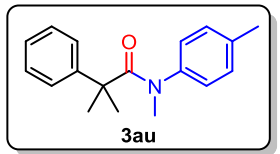

\section{$N$,2-dimethyl-2-phenyl- $N$-( $p$-tolyl)propenamide (3au)}

Yield $=70 \%, \mathrm{~b} / \mathrm{l}=93: 7,{ }^{1} \mathrm{H}$ NMR $\left(400 \mathrm{MHz}, \mathrm{CDCl}_{3}\right) \delta 7.18-6.46(\mathrm{~m}, 9 \mathrm{H}), 3.10$ (m, 3H), $2.26(\mathrm{~s}, 3 \mathrm{H}), 1.45(\mathrm{~s}, 6 \mathrm{H}) ;{ }^{13} \mathrm{C} \mathrm{NMR}\left(101 \mathrm{MHz}, \mathrm{CDCl}_{3}\right) \delta$ 176.4, 146.4, 141.3, 136.8, 129.2, 128.4, 126.2, 125.4, 47.6, 40.8, 28.7, 21.1. HRMS calcd. (ESI) $\mathrm{m} / \mathrm{z}$ for $\mathrm{C}_{18} \mathrm{H}_{21} \mathrm{NNaO}[\mathrm{M}+\mathrm{Na}]^{+}: 390.1515$, found: 290.1515 . 


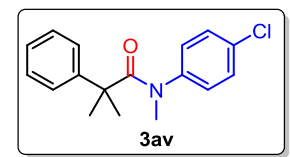

$N$-(4-chlorophenyl)- $N, 2$-dimethyl-2-phenylpropanamide (3av)

Yield $=71 \%, \mathrm{~b} / \mathrm{l}=90: 10,{ }^{1} \mathrm{H}$ NMR $\left(600 \mathrm{MHz}, \mathrm{CDCl}_{3}\right) \delta$ 7.22-7.04 (m, 7H), 6.57 (m, 2H), 3.06 (s, 3H), 1.47 (s, 6H); $\left.{ }^{13} \mathrm{C} \mathrm{NMR} \mathrm{(151} \mathrm{MHz,} \mathrm{CDCl}_{3}\right) \delta 176.3,146.0$ 142.5, 132.7, 129.8, 128.8, 128.6, 126.5, 125.4, 47.7, 40.6, 29.8, 28.6. HRMS calcd. (ESI) $\mathrm{m} / \mathrm{z}$ for $\mathrm{C}_{17} \mathrm{H}_{18} \mathrm{ClNNaO}[\mathrm{M}+\mathrm{Na}]^{+}: 310.0969$, found: 310.0969 .

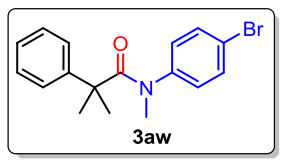

$N$-(4-bromophenyl)- $N$,2-dimethyl-2-phenylpropanamide (3aw)

Yield $=75 \%, \mathrm{~b} / \mathrm{l}=88: 12,{ }^{1} \mathrm{H}$ NMR $\left(600 \mathrm{MHz}, d^{6}\right.$-DMSO) $\delta$ 7.34-7.22 (m, 5H), 7.09 (s, 2H), 6.80 (s, 2H), 2.90 (s, 3H), 1.39 (s, 6H); ${ }^{13} \mathrm{C}$ NMR (151 MHz, $d^{6}$-DMSO) $\delta 174.9,145.8,143.5,131.5,130.1,128.6,126.4,125.0,119.5,47.0,28.4$. HRMS calcd. (ESI) $\mathrm{m} / \mathrm{z}$ for $\mathrm{C}_{17} \mathrm{H}_{18} \mathrm{BrNNaO}[\mathrm{M}+\mathrm{Na}]^{+}$: 354.0463 , found: 354.0461 .

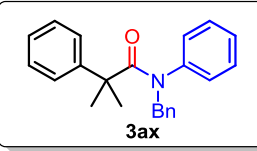

\section{$N$-benzyl-2-methyl- $N$,2-diphenylpropanamide (3ax)}

Yield $=61 \%, \mathrm{~b} / \mathrm{l}=86: 14,{ }^{1} \mathrm{H}$ NMR $\left(400 \mathrm{MHz} \mathrm{CDCl}_{3}\right) \delta$ 7.34-6.89 (m, 14H), $6.29(\mathrm{~s}, 2 \mathrm{H}), 4.79(\mathrm{~s}, 2 \mathrm{H}), 1.45(\mathrm{~s}, 6 \mathrm{H}) ;{ }^{13} \mathrm{C} \mathrm{NMR}\left(101 \mathrm{MHz}, \mathrm{CDCl}_{3}\right) \delta 176.1,146.2$, 141.8, 137.9, 130.2, 129.0, 128.3, 128.3, 128.1, 127.3, 127.1, 126.2, 125.6, 56.1, 47.9, 29.0. HRMS calcd. (ESI) m/z for $\mathrm{C}_{23} \mathrm{H}_{23} \mathrm{NNaO}[\mathrm{M}+\mathrm{Na}]^{+}$: 352.1671 , found: 352.1666 . 


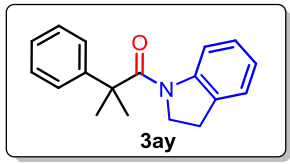

1-(indolin-1-yl)-2-methyl-2-phenylpropan-1-one (3ay)

Yield $=52 \%, \mathrm{~b} / \mathrm{l}=83: 17,{ }^{1} \mathrm{H}$ NMR $\left(400 \mathrm{MHz}, \mathrm{CDCl}_{3}\right) \delta 8.33(\mathrm{~d}, J=8.0 \mathrm{~Hz}, 1 \mathrm{H})$, 7.35-7.29 (m, 4H), 7.26-7.20 (m, 3H), $7.11(\mathrm{~d}, J=6.8 \mathrm{~Hz}, 1 \mathrm{H}), 7.00(\mathrm{t}, J=7.6 \mathrm{~Hz}$, $1 \mathrm{H}), 3.37(\mathrm{t}, J=8.0 \mathrm{~Hz}, 2 \mathrm{H}), 2.78(\mathrm{t}, J=8.0 \mathrm{~Hz}, 2 \mathrm{H}), 1.65(\mathrm{~s}, 6 \mathrm{H}) ;{ }^{13} \mathrm{C}$ NMR $(101$ $\left.\mathrm{MHz}, \mathrm{CDCl}_{3}\right) \delta 175.0,145.6,144.5,131.2,129.1,127.5,126.7,125.5,124.4,123.9$, 118.4, 49.0, 48.7, 28.9, 27.8. HRMS calcd. (ESI) $\mathrm{m} / \mathrm{z}$ for $\mathrm{C}_{18} \mathrm{H}_{19} \mathrm{NNaO}[\mathrm{M}+\mathrm{Na}]^{+}$: 288.1358 found: 288.1356.

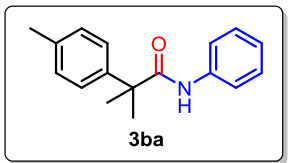

\section{2-methyl- $N$-phenyl-2-(p-tolyl)propanamide (3ba)}

Yield $=89 \% \mathrm{~b} / \mathrm{l}=98: 2,{ }^{1} \mathrm{H}$ NMR $\left(400 \mathrm{MHz}, \mathrm{CDCl}_{3}\right) \delta$ 7.35-7.30 (m, 4H), 7.23-7.17 (m, 4H), $7.00(\mathrm{t}, J=7.6 \mathrm{~Hz}, 1 \mathrm{H}), 6.89(\mathrm{~s}, 1 \mathrm{H}), 2.34(\mathrm{~s}, 3 \mathrm{H}), 1.62(\mathrm{~s}, 6 \mathrm{H})$; ${ }^{13} \mathrm{C} \mathrm{NMR}\left(101 \mathrm{MHz}, \mathrm{CDCl}_{3}\right) \delta 175.8,144.6,138.7,138.1,129.0,128.2,127.3,124.2$, 123.6, 119.7, 48.0, 27.1, 21.7. HRMS calcd. (ESI) $\mathrm{m} / \mathrm{z}$ for $\mathrm{C}_{16} \mathrm{H}_{17} \mathrm{NO}[\mathrm{M}+\mathrm{Na}]^{+}$: 262.1202, found: 262.1211 .

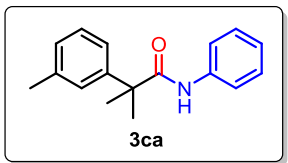

\section{2-methyl- $N$-phenyl-2-(m-tolyl)propanamide (3ca)}

Yield $=78 \%, \mathrm{~b} / \mathrm{l}>99: 1,{ }^{1} \mathrm{H}$ NMR $\left(400 \mathrm{MHz}, \mathrm{CDCl}_{3}\right) 7.35(\mathrm{~d}, J=7.6 \mathrm{~Hz}, 2 \mathrm{H})$, 7.30-7.21 (m, 5H), $7.12(\mathrm{~d}, J=7.2 \mathrm{~Hz}, 1 \mathrm{H}), 7.06-7.02(\mathrm{~m}, 1 \mathrm{H}), 6.83(\mathrm{~s}, 1 \mathrm{H}), 2.37$ (s, 3H), $1.65(\mathrm{~s}, 6 \mathrm{H}) ;{ }^{13} \mathrm{C}$ NMR (101 MHz, $\left.\mathrm{CDCl}_{3}\right) \delta 175.8,144.6,138.7,138.1,128.9$, 128.2, 127.3, 124.2, 123.6, 119.7, 48.0, 27.1, 21.7. HRMS calcd. (ESI) m/z for $\mathrm{C}_{17} \mathrm{H}_{19} \mathrm{NNaO}[\mathrm{M}+\mathrm{Na}]^{+}:$276.1358, found: 276.1370 . 


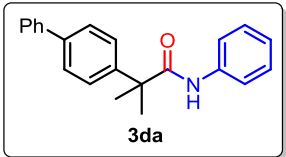

2-([1,1'-biphenyl]-4-yl)-2-methyl- $N$-phenylpropanamide (3da)

Yield $=95 \%, \mathrm{~b} / \mathrm{l}>99: 1,{ }^{1} \mathrm{H}$ NMR $\left(400 \mathrm{MHz}, \mathrm{CDCl}_{3}\right) \delta$ 7.64-7.60 (m, 4H), 7.53-7.50 (m, 2H), 7.47-7.43 (m, 2H), 7.39-7.33 (m, 3H), 7.28-7.24 (m, 2H), 7.07-7.03 (m, 1H), $6.88(\mathrm{~s}, 1 \mathrm{H}), 1.70(\mathrm{~s}, 6 \mathrm{H}) ;{ }^{13} \mathrm{C} \mathrm{NMR}\left(101 \mathrm{MHz}, \mathrm{CDCl}_{3}\right) \delta 175.6$, $143.7,140.4,140.3,138.1,129.0,129.0,127.7,127.6,127.1,127.1,124.3,119.8$, 48.0, 27.2. HRMS calcd. (ESI) $\mathrm{m} / \mathrm{z}$ for $\mathrm{C}_{22} \mathrm{H}_{21} \mathrm{NNaO}[\mathrm{M}+\mathrm{Na}]^{+}: 338.1515$, found: 338.1515 .

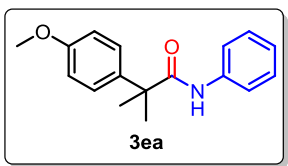

\section{2-(4-methoxyphenyl)-2-methyl- $N$-phenylpropanamide (3ea)}

Yield $=84 \%, \mathrm{~b} / \mathrm{l}>99: 1,{ }^{1} \mathrm{H}$ NMR $\left(400 \mathrm{MHz}, \mathrm{CDCl}_{3}\right) \delta$ 7.36-7.34 (m, 4H), 7.24 (t, $J=7.2 \mathrm{~Hz}, 2 \mathrm{H}), 7.06-7.02(\mathrm{~m}, 1 \mathrm{H}), 6.94-6.89(\mathrm{~m}, 3 \mathrm{H}), 3.81(\mathrm{~s}, 3 \mathrm{H}), 1.63(\mathrm{~s}, 6 \mathrm{H})$; ${ }^{13} \mathrm{C}$ NMR $\left(101 \mathrm{MHz}, \mathrm{CDCl}_{3}\right) \delta 176.0,158.8,138.1,136.5,128.9,127.7,124.1,119.7$, 114.3, 55.3, 47.4, 27.2. HRMS calcd. (ESI) $\mathrm{m} / \mathrm{z}$ for $\mathrm{C}_{17} \mathrm{H}_{19} \mathrm{NNaO}_{2}[\mathrm{M}+\mathrm{Na}]^{+}$: 292.1308, found: 292.1308 .

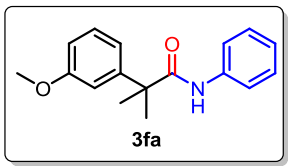

\section{2-(3-methoxyphenyl)-2-methyl- $N$-phenylpropanamide (3fa)}

Yield $=91 \%, \mathrm{~b} / \mathrm{l}>99: 1,{ }^{1} \mathrm{H}$ NMR $\left(400 \mathrm{MHz}, \mathrm{CDCl}_{3}\right) \delta$ 7.38-7.35 (m, 4H), 7.27-7.23 (m, 2H), $7.04(\mathrm{t}, J=2.8 \mathrm{~Hz}, 1 \mathrm{H}), 6.95-6.91(\mathrm{~m}, 2 \mathrm{H}), 6.85(\mathrm{~s}, 1 \mathrm{H}), 3.82(\mathrm{~s}$, 3H), $1.64(\mathrm{~s}, 6 \mathrm{H}) ;{ }^{13} \mathrm{C} \mathrm{NMR}\left(101 \mathrm{MHz}, \mathrm{CDCl}_{3}\right) \delta 175.5,160.1,146.3,138.1,130.1$, 128.9, 124.2, 119.8, 119.0, 113.0, 112.2, 55.4, 48.1, 27.1. HRMS calcd. (ESI) m/z for $\mathrm{C}_{17} \mathrm{H}_{19} \mathrm{NNaO}_{2}[\mathrm{M}+\mathrm{Na}]^{+}:$292.1308, found: 292.1306. 


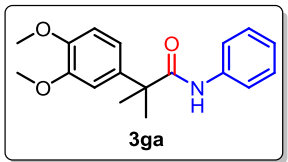

\section{2-(3,4-dimethoxyphenyl)-2-methyl- $N$-phenylpropanamide (3ga)}

Yield $=95 \%, \mathrm{~b} / \mathrm{l}>99: 1,{ }^{1} \mathrm{H} \mathrm{NMR}\left(400 \mathrm{MHz}, \mathrm{CDCl}_{3}\right) \delta 7.36(\mathrm{~d}, J=8.0 \mathrm{~Hz}, 2 \mathrm{H})$, 7.27-7.23 (m, 2H), 7.06-6.99 (m, 2H), 6.90 (d, J = 2.4 Hz, 2H), $6.88(\mathrm{~s}, 1 \mathrm{H}), 3.89$ (s, 3H), $3.86(\mathrm{~s}, 3 \mathrm{H}), 1.65(\mathrm{~s}, 6 \mathrm{H}) ;{ }^{13} \mathrm{C} \mathrm{NMR}\left(101 \mathrm{MHz}, \mathrm{CDCl}_{3}\right) \delta$ 175.9, 149.3, 148.4, 138.0, 137.1, 128.9, 124.2, 119.7, 118.5, 111.3, 110.1, 56.0, 55.9, 47.7, 27.2. HRMS calcd. (ESI) $\mathrm{m} / \mathrm{z}$ for $\mathrm{C}_{18} \mathrm{H}_{21} \mathrm{NNaO}_{3}[\mathrm{M}+\mathrm{Na}]^{+}: 322.1412$, found: 322.1412 .

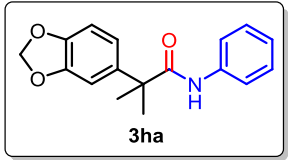

\section{2-(benzo[d][1,3]dioxol-5-yl)-2-methyl- $N$-phenylpropanamide (3ha)}

Yield $=83 \%$, b/l > 99:1; ${ }^{1} \mathrm{H}$ NMR $\left(400 \mathrm{MHz}, \mathrm{CDCl}_{3}\right) \delta 7.38(\mathrm{~d}, J=8.0 \mathrm{~Hz}, 2 \mathrm{H})$, $7.26(\mathrm{t}, J=7.6 \mathrm{~Hz}, 2 \mathrm{H}), 7.05(\mathrm{t}, J=7.4 \mathrm{~Hz}, 1 \mathrm{H}), 6.91-6.89(\mathrm{~m}, 3 \mathrm{H}), 6.83-6.81(\mathrm{~m}$, 1H), 5.97 (s, 2H), $1.62(\mathrm{~s}, 6 \mathrm{H}) ;{ }^{13} \mathrm{C} \mathrm{NMR}\left(101 \mathrm{MHz}, \mathrm{CDCl}_{3}\right) \delta$ 175.6, 148.3, 146.9, 138.6, 138.1, 129.0, 124.2, 119.7, 119.6, 108.5, 107.4, 101.4, 47.9, 27.3. HRMS calcd. (ESI) $\mathrm{m} / \mathrm{z}$ for $\mathrm{C}_{17} \mathrm{H}_{17} \mathrm{NNaO}_{3}[\mathrm{M}+\mathrm{Na}]^{+}:$306.1100, found: 306.1114 .

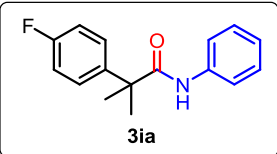

\section{2-(4-fluorophenyl)-2-methyl- $N$-phenylpropanamide (3ia)}

Yield $=70 \%, \mathrm{~b} / \mathrm{l}=94: 6,{ }^{1} \mathrm{H}$ NMR $\left(400 \mathrm{MHz}, \mathrm{CDCl}_{3}\right) \delta$ 7.42-7.34 (m, 4H), 7.27-7.23 (m, 2H), 7.09-7.03 (m, 3H), 6.87 (s, 1H ), 1.63 (s, 6H); ${ }^{13} \mathrm{C}$ NMR (101 MHz, $\left.\mathrm{CDCl}_{3}\right) \delta 175.3,161.9(\mathrm{~d}, J=245.2 \mathrm{~Hz}), 140.5,137.9,129.0,128.2(\mathrm{~d}, J=7.8 \mathrm{~Hz})$, 124.3, 119.8, $115.8(\mathrm{~d}, J=21.1 \mathrm{~Hz}), 47.6,27.2 ;{ }^{19} \mathrm{~F} \mathrm{NMR}\left(376 \mathrm{MHz}, \mathrm{CDCl}_{3}\right) \delta-131.8$. HRMS calcd. (ESI) m/z for $\mathrm{C}_{16} \mathrm{H}_{16} \mathrm{FNNaO}[\mathrm{M}+\mathrm{Na}]^{+}$: 280.1108, found: 280.1109 . 


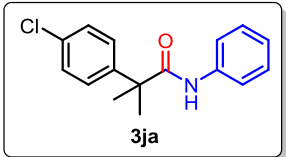

2-(4-chlorophenyl)-2-methyl- $N$-phenylpropanamide (3ja)

Yield $=73 \%, \mathrm{~b} / \mathrm{l}>99: 1,{ }^{1} \mathrm{H}$ NMR $\left(400 \mathrm{MHz}, \mathrm{CDCl}_{3}\right) \delta$ 7.37-7.35 (m, 6H), 7.28-7.24 (m, 2H), $7.07(\mathrm{t}, J=7.2 \mathrm{~Hz}, 1 \mathrm{H}), 6.81(\mathrm{~s}, 1 \mathrm{H}), 1.64(\mathrm{~s}, 6 \mathrm{H}) ;{ }^{13} \mathrm{C}$ NMR $(101$ $\left.\mathrm{MHz}, \mathrm{CDCl}_{3}\right) \delta 175.0,143.3,137.9,133.4,129.2,129.0,128.0,124.4,119.9,47.8$, 27.1. HRMS calcd. (ESI) $\mathrm{m} / \mathrm{z}$ for $\mathrm{C}_{16} \mathrm{H}_{16} \mathrm{ClNNaO}[\mathrm{M}+\mathrm{Na}]^{+}$: 296.0812, found: 296.0812.

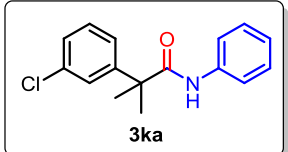

\section{2-(3-chlorophenyl)-2-methyl- $N$-phenylpropanamide (3ka)}

Yield $=86 \%$, b/l > 99:1, ${ }^{1} \mathrm{H}$ NMR $\left(400 \mathrm{MHz}, \mathrm{CDCl}_{3}\right) \delta 7.43(\mathrm{~s}, 1 \mathrm{H}), 7.37(\mathrm{~d}, J=$ $8.0 \mathrm{~Hz}, 2 \mathrm{H}), 7.32-7.24(\mathrm{~m}, 5 \mathrm{H}), 7.06(\mathrm{t}, J=7.2 \mathrm{~Hz}, 1 \mathrm{H}), 6.84(\mathrm{~s}, 1 \mathrm{H}), 1.64(\mathrm{~s}, 6 \mathrm{H})$; ${ }^{13} \mathrm{C} \mathrm{NMR}\left(101 \mathrm{MHz}, \mathrm{CDCl}_{3}\right) \delta 174.0,146.2,137.9,135.0,130.3,129.0,127.7,126.6$, 124.9, 124.5, 119.9, 48.1, 27.0. HRMS calcd. (ESI) m/z for $\mathrm{C}_{16} \mathrm{H}_{16} \mathrm{ClNNaO}[\mathrm{M}+\mathrm{Na}]^{+}$: 296.0812, found: 296.0824 .

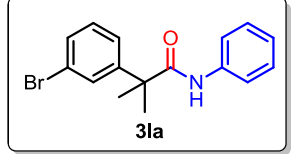

\section{2-(4-bromophenyl)-2-methyl- $N$-phenylpropanamide (3la)}

Yield $=92 \%, \mathrm{~b} / \mathrm{l}>99: 1,{ }^{1} \mathrm{H} \mathrm{NMR}\left(400 \mathrm{MHz}, \mathrm{CDCl}_{3}\right) \delta 7.58(\mathrm{t}, J=1.6 \mathrm{~Hz} 1 \mathrm{H})$, $7.44(\mathrm{~d}, J=8.0 \mathrm{~Hz}, 1 \mathrm{H}), 7.37(\mathrm{t}, J=8.0 \mathrm{~Hz}, 3 \mathrm{H}), 7.29-7.24(\mathrm{~m}, 3 \mathrm{H}), 7.07$ (t, $J=7.2$ $\mathrm{Hz}, 1 \mathrm{H}), 6.82(\mathrm{~s}, 1 \mathrm{H}), 1.64(\mathrm{~s}, 6 \mathrm{H}) ;{ }^{13} \mathrm{C} \mathrm{NMR}\left(101 \mathrm{MHz}, \mathrm{CDCl}_{3}\right) \delta$ 174.7, 147.1, 137.8, 130.6, 129.4, 129.0, 125.4, 124.4, 123.2, 119.9, 48.0, 27.0. HRMS calcd. (ESI) $\mathrm{m} / \mathrm{z}$ for $\mathrm{C}_{16} \mathrm{H}_{16} \mathrm{BrNNaO}[\mathrm{M}+\mathrm{Na}]^{+}: 340.0307$, found: 340.0317 . 


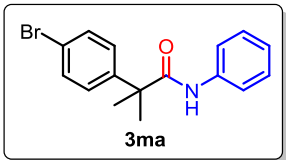

\section{2-(3-bromophenyl)-2-methyl- $N$-phenylpropanamide (3ma)}

Yield $=72 \%, \mathrm{~b} / \mathrm{l}>$ 99:1, ${ }^{1} \mathrm{H}$ NMR $\left(400 \mathrm{MHz}, \mathrm{CDCl}_{3}\right)$ 7.51-7.48 (m, 2H), 7.37-7.34 (m, 2H), 7.30-7.03 (m, 4H), 7.08-7.03 (m, 1H), $6.87(\mathrm{~s}, 1 \mathrm{H}), 1.61(\mathrm{~s}, 6 \mathrm{H})$; ${ }^{13} \mathrm{C}$ NMR $\left(101 \mathrm{MHz}, \mathrm{CDCl}_{3}\right) \delta 174.9,143.8,137.9,132.2,129.0,128.3,124.4,121.5$, 119.9, 47.9, 27.1. HRMS calcd. (ESI) $\mathrm{m} / \mathrm{z}$ for $\mathrm{C}_{16} \mathrm{H}_{16} \mathrm{BrNNaO}[\mathrm{M}+\mathrm{Na}]^{+}$: 340.0307, found: 340.0319 .

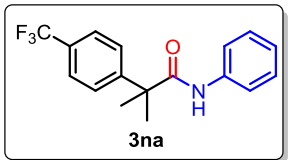

\section{2-methyl- $N$-phenyl-2-(4-(trifluoromethyl)phenyl)propanamide (3na)}

Yield $=73 \%, \mathrm{~b} / \mathrm{l}=97: 3,{ }^{1} \mathrm{H} \mathrm{NMR}\left(400 \mathrm{MHz}, \mathrm{CDCl}_{3}\right) \delta 7.64(\mathrm{~d}, J=8.0 \mathrm{~Hz}, 2 \mathrm{H})$, $7.54(\mathrm{~d}, J=8.4 \mathrm{~Hz}, 2 \mathrm{H}), 7.38-7.36(\mathrm{~m}, 2 \mathrm{H}), 7.28-7.24(\mathrm{~m}, 2 \mathrm{H}), 7.10-7.054(\mathrm{~m}, 1 \mathrm{H})$, $6.86(\mathrm{~s}, 1 \mathrm{H}), 1.67(\mathrm{~s}, 6 \mathrm{H}) ;{ }^{13} \mathrm{C} \mathrm{NMR}\left(101 \mathrm{MHz}, \mathrm{CDCl}_{3}\right) \delta 174.5,149.0,137.8,130.2$, $129.6(\mathrm{~d}, J=32.4 \mathrm{~Hz}), 129.0,126.9,126.0(\mathrm{~d}, J=32.4 \mathrm{~Hz}), 125.5,124.4(\mathrm{~d}, J=170.3$ $\mathrm{Hz}), 120.0,48.2,27.0 ;{ }^{19} \mathrm{~F}$ NMR $\left(376 \mathrm{MHz}, \mathrm{CDCl}_{3}\right) \delta$-62.42. HRMS calcd. (ESI) m/z for $\mathrm{C}_{17} \mathrm{H}_{16} \mathrm{~F}_{3} \mathrm{NNaO}$ [M+Na]+: 330.1076, found: 330.1085 .

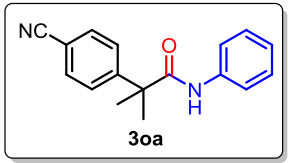

\section{2-(4-cyanophenyl)-2-methyl- $N$-phenylpropanamide (3oa)}

Yield $=94 \%, \mathrm{~b} / \mathrm{l}>99: 1,{ }^{1} \mathrm{H} \mathrm{NMR}\left(400 \mathrm{MHz}, \mathrm{CDCl}_{3}\right) \delta 7.66(\mathrm{~d}, J=8.4 \mathrm{~Hz}, 2 \mathrm{H})$, $7.55(\mathrm{~d}, J=8.4 \mathrm{~Hz}, 2 \mathrm{H}), 7.39(\mathrm{~d}, J=8.0 \mathrm{~Hz}, 2 \mathrm{H}), 7.30-7.26(\mathrm{~m}, 2 \mathrm{H}), 7.09(\mathrm{t}, J=7.4$ $\mathrm{Hz}, 1 \mathrm{H}), 6.86(\mathrm{~s}, 1 \mathrm{H}), 1.67(\mathrm{~s}, 6 \mathrm{H}) ;{ }^{13} \mathrm{C} \mathrm{NMR}\left(101 \mathrm{MHz}, \mathrm{CDCl}_{3}\right) \delta 173.9,150.3$, 137.7, 132.8, 129.1, 127.3, 124.7, 120.0, 118.6, 111.3, 48.4, 26.9. HRMS calcd. (ESI) $\mathrm{m} / \mathrm{z}$ for $\mathrm{C}_{17} \mathrm{H}_{16} \mathrm{~N}_{2} \mathrm{NaO}[\mathrm{M}+\mathrm{Na}]^{+}:$287.1154, found: 287.1157 . 


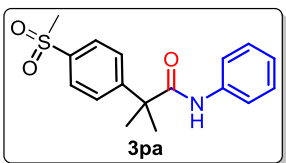

2-methyl-2-(4-(methylsulfonyl)phenyl)- $N$-phenylpropanamide (3pa)

Yield $=93 \%, \mathrm{~b} / \mathrm{l}>99: 1 ;{ }^{1} \mathrm{H}$ NMR (400 MHz, $d^{6}$-DMSO) $\delta 9.24(\mathrm{~s}, 1 \mathrm{H}), 7.93(\mathrm{~d}$, $J=8.4 \mathrm{~Hz}, 2 \mathrm{H}), 7.65-7.60(\mathrm{~m}, 4 \mathrm{H}), 7.28(\mathrm{t}, J=7.8 \mathrm{~Hz}, 2 \mathrm{H}), 7.04(\mathrm{t}, J=7.4 \mathrm{~Hz}, 1 \mathrm{H})$, $3.21(\mathrm{~s}, 3 \mathrm{H}), 1.61(\mathrm{~s}, 6 \mathrm{H}) ;{ }^{13} \mathrm{C}$ NMR (101 MHz, $d^{6}$-DMSO) $\delta 173.9,151.7,139.1$, 138.9, 128.5, 127.1, 127.0, 123.5, 120.4, 47.8, 43.6, 26.6. HRMS calcd. (ESI) m/z for $\mathrm{C}_{17} \mathrm{H}_{19} \mathrm{~N}_{3} \mathrm{NaOS}[\mathrm{M}+\mathrm{Na}]^{+}:$340.0977, found: 340.0977 .

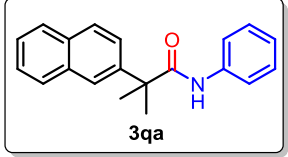

2-methyl-2-(naphthalen-2-yl)- $N$-phenylpropanamide (3qa)

Yield $=95 \%, \mathrm{~b} / \mathrm{l}>99: 1 ;{ }^{1} \mathrm{H}$ NMR $\left(400 \mathrm{MHz}, \mathrm{CDCl}_{3}\right) \delta$ 7.69-7.82 (m, 4H), 7.52-7.45 (m, 3H),7.32 (d, $J=8.0 \mathrm{~Hz}, 2 \mathrm{H}) 7.20(\mathrm{t}, J=7.2 \mathrm{~Hz}, 2 \mathrm{H}), 7.01(\mathrm{t}, J=7.2 \mathrm{~Hz}$, 2H), $6.86(\mathrm{~s}, 1 \mathrm{H}), 1.74(\mathrm{~s}, 6 \mathrm{H}) ;{ }^{13} \mathrm{C} \mathrm{NMR}\left(101 \mathrm{MHz}, \mathrm{CDCl}_{3}\right) \delta$ 175.5, 142.1, 138.0, 133.4, 132.8, 128.9, 128.2, 127.7, 126.7, 126.4, 125.4, 124.6, 124.2, 119.8, 48.4, 27.1. HRMS calcd. (ESI) m/z for $\mathrm{C}_{20} \mathrm{H}_{19} \mathrm{NNaO}[\mathrm{M}+\mathrm{Na}]^{+}: 312.1358$, found: 312.1351 .

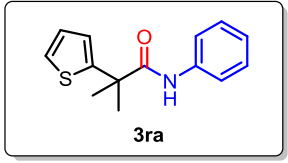

\section{2-methyl- $N$-phenyl-2-(thiophen-2-yl)propanamide (3ra)}

Yield $=48 \%, \mathrm{~b} / \mathrm{l}>99: 1 ;{ }^{1} \mathrm{H} \mathrm{NMR}\left(400 \mathrm{MHz}, \mathrm{CDCl}_{3}\right) \delta 7.36(\mathrm{~d}, J=8.0 \mathrm{~Hz}, 2 \mathrm{H})$, $7.33(\mathrm{~d}, J=4.8 \mathrm{~Hz}, 1 \mathrm{H}), 7.28-7.25(\mathrm{~m}, 2 \mathrm{H}), 7.18(\mathrm{~s}, 1 \mathrm{H}), 7.10(\mathrm{~d}, J=2.8 \mathrm{~Hz}, 1 \mathrm{H})$, 7.08-7.05 (m, 2H), 1.75 (s, 6H); ${ }^{13} \mathrm{C}$ NMR (101 MHz, $\left.\mathrm{CDCl}_{3}\right) \delta$ 174.2, 149.6, 137.9, 129.0, 127.5, 125.5, 125.1, 124.4, 119.8, 46.4, 28.3. HRMS calcd. (ESI) m/z for $\mathrm{C}_{14} \mathrm{H}_{15} \mathrm{NNaOS}[\mathrm{M}+\mathrm{Na}]^{+}$: 268.0766, found: 268.0766. 


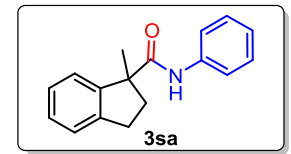

\section{$N, 1-d i m e t h y l-N$-phenyl-2,3-dihydro-1H-indene-1-carboxamide (3sa)}

Yield $=75 \%, \mathrm{~b} / \mathrm{l}>99: 1,{ }^{1} \mathrm{H}$ NMR $\left(600 \mathrm{MHz}, \mathrm{CDCl}_{3}\right) \delta 7.36-7.31(\mathrm{~m}, 6 \mathrm{H})$, 7.26-7.24 (m, 2H), $7.06(\mathrm{t}, J=6.0 \mathrm{~Hz}, 2 \mathrm{H}), 3.03-2.96(\mathrm{~m}, 2 \mathrm{H}), 2.73-2.70(\mathrm{~m}, 1 \mathrm{H})$, 2.14-2.09 (m, 1H), 1.65 (s, 3H); ${ }^{13} \mathrm{C}$ NMR (151 MHz, $\left.\mathrm{CDCl}_{3}\right) \delta$ 174.9, 145.7, 144.9, 137.8, 129.0, 128.4, 127.5, 125.7, 124.3, 123.7, 119.7, 56.7, 40.2, 30.6, 24.5. HRMS calcd. (ESI) $\mathrm{m} / \mathrm{z}$ for $\mathrm{C}_{17} \mathrm{H}_{17} \mathrm{NNaO}[\mathrm{M}+\mathrm{Na}]^{+}:$288.1358, found: 288.1358 .

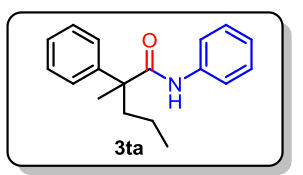

\section{2-methyl- $N, 2$-diphenylpentanamide (3ta)}

Yield $=51 \%, \mathrm{~b} / \mathrm{l}=88: 12,{ }^{1} \mathrm{H}$ NMR $\left(400 \mathrm{MHz}, \mathrm{CDCl}_{3}\right) \delta$ 7.41-7.34 (m, 5H), 7.32-7.29 (m, 1H), 7.28-7.24 (m, 3H), 7.07-7.03 (m, 1H), $6.79(\mathrm{~s}, 1 \mathrm{H}), 2.11-1.98(\mathrm{~m}$, $2 \mathrm{H}), 1.62(\mathrm{~s}, 3 \mathrm{H}), 1.32-1.26(\mathrm{~m}, 1 \mathrm{H}), 1.20-1.12(\mathrm{~m}, 1 \mathrm{H}), 0.92(\mathrm{t}, J=6.8 \mathrm{~Hz}, 3 \mathrm{H}) ;{ }^{13} \mathrm{C}$ NMR $\left(101 \mathrm{MHz}, \mathrm{CDCl}_{3}\right) \delta 175.4,143.9,138.1,129.1,129.0,127.4,127.1,124.2$, 119.8, 51.8, 41.3, 24.1, 17.9, 14.8. HRMS calcd. (ESI) $\mathrm{m} / \mathrm{z}$ for $\mathrm{C}_{17} \mathrm{H}_{17} \mathrm{NNaO}[\mathrm{M}+\mathrm{Na}]^{+}$: 290.1515, found: 290.1515 .

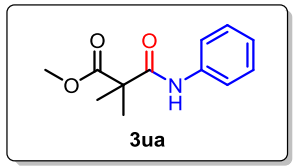

methyl 2,2-dimethyl-3-oxo-3-(phenylamino)propanoate (3ua)

Yield $=86 \%$, b/l $>99: 1 ;{ }^{1} \mathrm{H}$ NMR $\left(400 \mathrm{MHz}, \mathrm{CDCl}_{3}\right) \delta 8.55(\mathrm{~s}, 1 \mathrm{H}), 7.53(\mathrm{~d}, J=$ $7.6 \mathrm{~Hz}, 2 \mathrm{H}), 7.32(\mathrm{t}, J=7.6 \mathrm{~Hz}, 2 \mathrm{H}), 7.11(\mathrm{t}, J=7.2 \mathrm{~Hz}, 1 \mathrm{H}), 3.79(\mathrm{~s}, 3 \mathrm{H}), 1.56(\mathrm{~s}$, $6 \mathrm{H}) ;{ }^{13} \mathrm{C}$ NMR $\left(101 \mathrm{MHz}, \mathrm{CDCl}_{3}\right) \delta 176.1,169.7,137.9,129.1,124.5,120.1,53.1$, 50.6, 24.0. HRMS calcd. (ESI) $\mathrm{m} / \mathrm{z}$ for $\mathrm{C}_{12} \mathrm{H}_{15} \mathrm{NNaO}_{3}[\mathrm{M}+\mathrm{Na}]^{+}$: 244.0944, found: 244.0954 . 


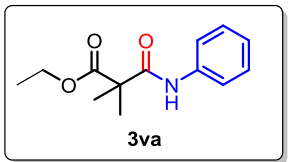

ethyl 2,2-dimethyl-3-oxo-3-(phenylamino)propanoate (3va)

Yield $=85 \%, \mathrm{~b} / \mathrm{l}>99: 1,{ }^{1} \mathrm{H} \mathrm{NMR}\left(600 \mathrm{MHz}, \mathrm{CDCl}_{3}\right) \delta 8.63(\mathrm{~s}, 1 \mathrm{H}), 7.53(\mathrm{~d}$, $4.0 \mathrm{~Hz}, 2 \mathrm{H}), 7.32(\mathrm{t}, J=8.1 \mathrm{~Hz}, 2 \mathrm{H}), 7.10(\mathrm{t}, J=10.5 \mathrm{~Hz}, 1 \mathrm{H}), 4.24(\mathrm{q}, J=6.6 \mathrm{~Hz}$, 2H), $1.55(\mathrm{~s}, 6 \mathrm{H}), 1.30(\mathrm{t}, J=6.9 \mathrm{~Hz}, 3 \mathrm{H}) ;{ }^{13} \mathrm{C} \mathrm{NMR}\left(151 \mathrm{MHz}, \mathrm{CDCl}_{3}\right) \delta$ 175.6, 169.2, 137.9, 129.0, 124.4, 120.0, 62.1, 50.5, 24.0, 14.1. HRMS calcd. (ESI) m/z for $\mathrm{C}_{13} \mathrm{H}_{17} \mathrm{NNaO}_{3}[\mathrm{M}+\mathrm{Na}]+:$ 258.1100, found:258.1101.

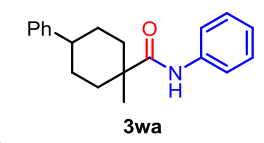

methyl- $N$,4-diphenylcyclohexane-1-carboxamide (3wa)

Yield $=52 \%, \mathrm{~b} / \mathrm{l}=95: 5,{ }^{1} \mathrm{H} \mathrm{NMR}\left(400 \mathrm{MHz}, \mathrm{CDCl}_{3}\right) \delta 7.55(\mathrm{~d}, J=8.0 \mathrm{~Hz}, 2 \mathrm{H})$, 7.39 (s, 1H), 7.34-7.30 (m, 4H), 7.25-7.16 (m, 3H), $7.10(\mathrm{t}, J=7.6 \mathrm{~Hz}, 1 \mathrm{H}), 2.59-2.48$ $(\mathrm{m}, 1 \mathrm{H}), 1.95-1.86(\mathrm{~m}, 5 \mathrm{H}), 1.78-1.67(\mathrm{~m}, 3 \mathrm{H}), 1.41(\mathrm{~s}, 3 \mathrm{H}) ;{ }^{13} \mathrm{C}$ NMR $(101 \mathrm{MHz}$, $\left.\mathrm{CDCl}_{3}\right) \delta 177.1,146.7,138.2,129.1,128.5,126.9,126.3,124.4,120.2,43.8,42.2$, 34.5, 29.3, 20.8. HRMS calcd. (ESI) $\mathrm{m} / \mathrm{z}$ for $\mathrm{C}_{20} \mathrm{H}_{23} \mathrm{NNaO}[\mathrm{M}+\mathrm{Na}]^{+}: 316.1671$, found: 316.1677 .

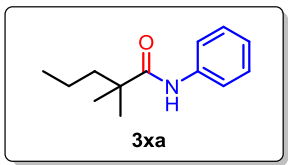

\section{2,2-dimethyl- $N$-phenylpentanamide (3xa)}

Yield $=60 \%, \mathrm{~b} / \mathrm{l}=94: 6 ;{ }^{1} \mathrm{H} \mathrm{NMR}\left(400 \mathrm{MHz}, \mathrm{CDCl}_{3}\right) \delta 7.52(\mathrm{~d}, J=8.0 \mathrm{~Hz}, 2 \mathrm{H})$, $7.38(\mathrm{~s}, 1 \mathrm{H}), 7.29(\mathrm{t}, J=7.8 \mathrm{~Hz}, 2 \mathrm{H}), 7.08(\mathrm{t}, J=7.2 \mathrm{~Hz}, 1 \mathrm{H}), 1.60-1.55(\mathrm{~m}, 2 \mathrm{H})$, 1.37-1.29 (m, 2H), $1.27(\mathrm{~s}, 6 \mathrm{H}), 0.91(\mathrm{t}, J=7.2 \mathrm{~Hz}, 3 \mathrm{H}) ;{ }^{13} \mathrm{C} \mathrm{NMR}\left(101 \mathrm{MHz}, \mathrm{CDCl}_{3}\right)$ $\delta 176.2,138.1,129.1,124.3,120.1,44.0,43.2,25.7,18.3,14.7$. HRMS calcd. (ESI) $\mathrm{m} / \mathrm{z}$ for $\mathrm{C}_{13} \mathrm{H}_{19} \mathrm{NNaO}[\mathrm{M}+\mathrm{Na}]^{+}: 228.1358$, found: 228.1358 . 


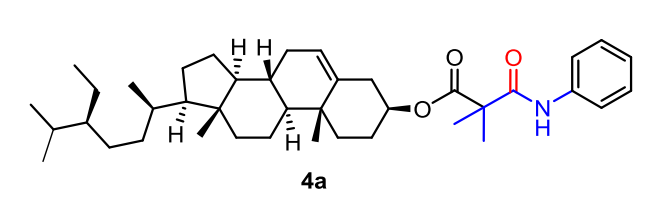

(3S,8S,9S,10R,13R,14S,17R)-17-((2R,5S)-5-ethyl-6-methylheptan-2-yl)-10,13-dim ethyl-2,3,4,7,8,9,10,11,12,13,14,15,16,17-tetradecahydro-1H-cyclopenta[a]phenan thren-3-yl 2,2-dimethyl-3-oxo-3-(phenylamino)propanoate (4a)

Yield $=65 \%$, b/l $>20: 1$ (based on ${ }^{1} \mathrm{H}$ NMR), ${ }^{1} \mathrm{H}$ NMR $\left(400 \mathrm{MHz}, \mathrm{CDCl}_{3}\right) \delta 8.65$ (s, 1H), 7.54-7.51 (m, 2H), 7.34-7.29 (m, 2H), 7.12-7.08 (m, 1H), 5.39 (d, J = 4.4 Hz, 1H), 4.74-4.66 (m, 1H), 2.35 (d, $J=7.2 \mathrm{~Hz}, 2 \mathrm{H}), 2.03-1.95(\mathrm{~m}, 2 \mathrm{H}), 1.90-1.82$ (m, $3 \mathrm{H}), 1.69-1.62(\mathrm{~m}, 2 \mathrm{H}), 1.59-1.57(\mathrm{~m}, 1 \mathrm{H}), 1.54(\mathrm{~s}, 6 \mathrm{H}), 1.51-1.43(\mathrm{~m}, 4 \mathrm{H}), 1.37-1.30$ (m, 2H), 1.28-1.19 (m, 3H), 1.20-1.10 (m, 6H), 1.02 (s, 3H), $0.96(\mathrm{~d}, J=6.0 \mathrm{~Hz}, 1 \mathrm{H})$, $0.92(\mathrm{~d}, J=6.4 \mathrm{~Hz}, 3 \mathrm{H}), 0.86-0.81(\mathrm{~m}, 10 \mathrm{H}), 0.68(\mathrm{~s}, 3 \mathrm{H}) ;{ }^{13} \mathrm{C}$ NMR $(101 \mathrm{MHz}$, $\left.\mathrm{CDCl}_{3}\right) \delta 175.0,170.0,139.3,138.0,129.1,124.4,123.2,120.0,75.8,56.8,56.2,50.6$, $50.1,45.9,42.4,39.8,37.9,37.0,36.7,36.3,34.0,32.0,31.9,29.3,28.4,27.7,26.2$, 24.4, 24.1, 24.0, 23.2 21.2, 20.0, 19.4, 19.2, 18.9, 12.1, 12.0. $\mathrm{C}_{39} \mathrm{H}_{59} \mathrm{NNaO}_{3}[\mathrm{M}+\mathrm{Na}]^{+}$: 626.4543, found: 626.4513 .

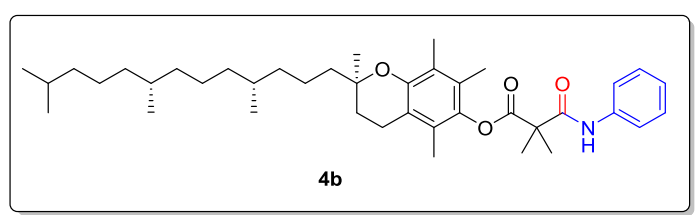

(R)-2,5,7,8-tetramethyl-2-((4R,8R)-4,8,12-trimethyltridecyl)chroman-6-yl 2,2-dimethyl-3-oxo-3-(phenylamino)propanoate (4b)

Yield $=89 \%, \mathrm{~b} / \mathrm{l}>20: 1$ (based on $\left.{ }^{1} \mathrm{H} \mathrm{NMR}\right),{ }^{1} \mathrm{H}$ NMR $\left(600 \mathrm{MHz}, \mathrm{CDCl}_{3}\right) \delta 8.80$ (s, 1H), $7.54(\mathrm{~d}, J=8.4 \mathrm{~Hz}, 2 \mathrm{H}), 7.32(\mathrm{t}, J=7.5 \mathrm{~Hz}, 2 \mathrm{H}), 7.11(\mathrm{t}, J=7.2 \mathrm{~Hz}, 1 \mathrm{H})$, $2.59(\mathrm{t}, J=6.6 \mathrm{~Hz}, 2 \mathrm{H}), 2.09(\mathrm{~s}, 3 \mathrm{H}), 2.00(\mathrm{~s}, 3 \mathrm{H}), 1.95(\mathrm{~s}, 3 \mathrm{H}), 1.78(\mathrm{~s}, 6 \mathrm{H})$, 1.58-1.50 (m, 3H), $1.37(\mathrm{~s}, 3 \mathrm{H}), 1.26-1.22(\mathrm{~m}, 11 \mathrm{H}), 1.14-1.06(\mathrm{~m}, 7 \mathrm{H}), 0.87-0.84(\mathrm{~m}$, $14 \mathrm{H}) ;{ }^{13} \mathrm{C} \mathrm{NMR}\left(151 \mathrm{MHz}, \mathrm{CDCl}_{3}\right) \delta 174.8,169.4,149.8$ 140.2, 137.8, 129.1, 126.5, 124.8, 124.6, 123.5, 120.1, 117.7, 75.3, 50.7, 40.6, 39.5, 37.5, 37.4, 32.9, 32.9, 32.8, 31.3, 31.0, 28.0, 24.9, 24.6, 24.4, 23.7, 22.9, 22.8, 21.1, 20.7, 19.9, 19.8, 13.0, 12.1, 12.0. HRMS calcd. (ESI) $\mathrm{m} / \mathrm{z}$ for $\mathrm{C}_{40} \mathrm{H}_{61} \mathrm{NNaO}_{4}[\mathrm{M}+\mathrm{Na}]^{+}$: 642.4492, found: 642.4463. 


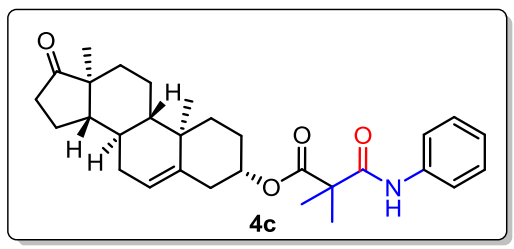

$(3 S, 8 R, 9 S, 10 R, 13 S, 14 S)-10,13-d i m e t h y l-17-0 x 0-2,3,4,7,8,9,10,11,12,13,14,15,16,17$ -tetradecahydro-1H-cyclopenta[a]phenanthren-3-yl

2,2-dimethyl-3-oxo-3-(phenylamino)propanoate (4c)

Yield $=86 \%$, b/l $>20: 1$ (based on ${ }^{1} \mathrm{H}$ NMR), ${ }^{1} \mathrm{H}$ NMR $\left(400 \mathrm{MHz}, \mathrm{CDCl}_{3}\right) \delta 8.60$ (s, 1H), $7.53(\mathrm{~d}, J=8.0 \mathrm{~Hz}, 2 \mathrm{H}), 7.32(\mathrm{t}, J=7.6 \mathrm{~Hz}, 2 \mathrm{H}), 7.11(\mathrm{t}, J=7.6 \mathrm{~Hz}, 1 \mathrm{H})$, $5.43(\mathrm{~d}, J=4.4 \mathrm{~Hz}, 1 \mathrm{H}), 4.71-7.66(\mathrm{~m}, 1 \mathrm{H}), 2.46$ (dd, $J=19.2,8.8 \mathrm{~Hz}, 1 \mathrm{H}), 2.37$ (d, $J$ $=7.6 \mathrm{~Hz}, 2 \mathrm{H}), 2.14-2.05(\mathrm{~m}, 2 \mathrm{H}), 1.99-1.84(\mathrm{~m}, 4 \mathrm{H}), 1.69-1.65(\mathrm{~m}, 4 \mathrm{H}), 1.55(\mathrm{~s}, 4 \mathrm{H})$, 1.33-1.26 (m, 4H), 1.20-1.14 (m, 2H), 1.06 (s, 3H), 0.89 (s, 3H); ${ }^{13} \mathrm{C}$ NMR (101 MHz, $\left.\mathrm{CDCl}_{3}\right) \delta 175.0,169.9,139.6,137.9,129.1,124.4,122.4,120.0,75.5,51.8,50.6,50.2$, 47.6, 37.9, 36.9, 36.8, 35.9, 31.5, 31.5, 30.9, 29.8, 27.6, 24.0, 24.0, 22.0, 20.4, 19.5, 13.6. $\mathrm{C}_{29} \mathrm{H}_{37} \mathrm{NNaO}_{4}[\mathrm{M}+\mathrm{Na}]^{+}: 500.2771$, found: 500.2762 .

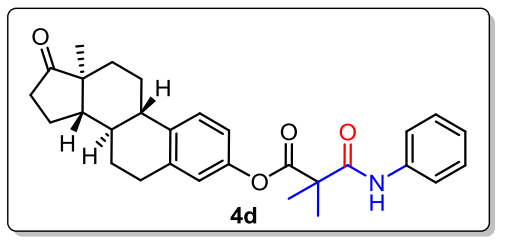

$(8 R, 9 S, 13 S, 14 S)$-13-methyl-17-oxo-7,8,9,11,12,13,14,15,16,17-decahydro-6H-cyclo penta[a]phenanthren-3-yl 2,2-dimethyl-3-oxo-3-(phenylamino)propanoate (4d)

Yield $=61 \%$, b/l $>20: 1$ (based on ${ }^{1} \mathrm{H}$ NMR), ${ }^{1} \mathrm{H}$ NMR $\left(400 \mathrm{MHz}, \mathrm{CDCl}_{3}\right) \delta 8.52$ (s, 1H), $7.54(\mathrm{~d}, J=8.0 \mathrm{~Hz}, 2 \mathrm{H}), 7.35-7.30(\mathrm{~m}, 3 \mathrm{H}), 7.12(\mathrm{t}, J=7.2 \mathrm{~Hz} 1 \mathrm{H}), 6.86(\mathrm{~d}, J$ $=8.4 \mathrm{~Hz}, 1 \mathrm{H}), 6.82(\mathrm{~s}, 1 \mathrm{H}), 3.48(\mathrm{~d}, J=0.4 \mathrm{~Hz}, 1 \mathrm{H}) 2.92-2.90(\mathrm{~m}, 2 \mathrm{H}), 2.51(\mathrm{dd}, J=$ $18.8,8.8 \mathrm{~Hz}, 1 \mathrm{H}), 2.42-2.38$ (m, 1H), 2.30-2.26 (m, 1H), 2.19-2.12 (m, 1H), 2.10-1.95 $(\mathrm{m}, 3 \mathrm{H}), 1.71(\mathrm{~s}, 6 \mathrm{H}), 1.66-1.60(\mathrm{~m}, 2 \mathrm{H}), 1.57-1.42(\mathrm{~m}, 4 \mathrm{H}), 0.91(\mathrm{~s}, 3 \mathrm{H}) ;{ }^{13} \mathrm{C} \mathrm{NMR}$ $\left(151 \mathrm{MHz}, \mathrm{CDCl}_{3}\right) \delta 221.0,174.7,169.4,148.4,138.4,138.1,137.8,129.1,126.7$, 124.6, 121.3, 120.1, 118.4, 50.8, 50.4, 48.0, 44.2, 38.0, 35.9, 31.6, 29.5, 26.4, 25.8, 24.0, 21.7, 13.9. HRMS calcd. (ESI) $\mathrm{m} / \mathrm{z}$ for $\mathrm{C}_{29} \mathrm{H}_{33} \mathrm{NNaO}_{4}[\mathrm{M}+\mathrm{Na}]^{+}:$482.2301, found: 482.2295 . 


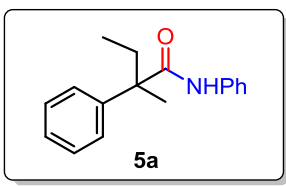

\section{2-methyl- $N$,2-diphenylbutanamide (5a)}

Yield $=56 \%, \mathrm{~b} / \mathrm{l}=98: 2,{ }^{1} \mathrm{H}$ NMR $\left(600 \mathrm{MHz}, d^{6}\right.$-DMSO) $\delta 9.06(\mathrm{~s}, 1 \mathrm{H}), 7.58(\mathrm{~d}$, $J=8.4 \mathrm{~Hz}, 2 \mathrm{H}), 7.34-7.33(\mathrm{~m}, 4 \mathrm{H}), 7.27-7.24(\mathrm{~m}, 3 \mathrm{H}), 7.01(\mathrm{t}, J=6.6 \mathrm{~Hz}, 1 \mathrm{H})$, 2.17-2.14 (m, 1H), 1.94-1.90 (m, 1H), $1.50(\mathrm{~s}, 3 \mathrm{H}), 0.78(\mathrm{t}, J=7.2 \mathrm{~Hz}, 3 \mathrm{H}) ;{ }^{13} \mathrm{C}$ NMR $\left(101 \mathrm{MHz}, \mathrm{CDCl}_{3}\right) \delta 175.3,143.5,138.1,129.0,129.0,127.4,127.1,124.2$, 119.8, 52.0, 31.6, 23.4, 9.0. HRMS calcd. (ESI) $\mathrm{m} / \mathrm{z}$ for $\mathrm{C}_{17} \mathrm{H}_{19} \mathrm{NNaO}[\mathrm{M}+\mathrm{H}]^{+}$: 254.1539, found: 254.1539

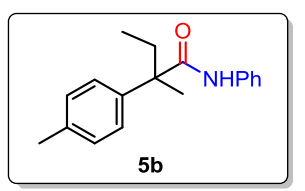

\section{2-methyl- $N$-phenyl-2-(p-tolyl)butanamide (5b)}

Yield $=66 \%, \mathrm{~b} / \mathrm{l}=85: 15,{ }^{1} \mathrm{H}$ NMR $\left(600 \mathrm{MHz}, d^{6}-\mathrm{DMSO}\right) \delta 8.99(\mathrm{~s}, 1 \mathrm{H}), 7.57(\mathrm{~d}$, $J=7.8 \mathrm{~Hz}, 2 \mathrm{H}), 7.26-7.23(\mathrm{~m}, 2 \mathrm{H}), 7.20(\mathrm{~d}, J=8.4 \mathrm{~Hz}, 2 \mathrm{H}), 7.14(\mathrm{~d}, J=7.8 \mathrm{~Hz}, 2 \mathrm{H})$, $7.01(\mathrm{t}, J=7.2 \mathrm{~Hz}, 1 \mathrm{H}), 2.27(\mathrm{~s}, 3 \mathrm{H}), 2.15-2.10(\mathrm{~m}, 1 \mathrm{H}), 1.96-1.87(\mathrm{~m}, 1 \mathrm{H}), 1.48$ (s, $3 \mathrm{H}), 0.77(\mathrm{t}, J=7.2 \mathrm{~Hz}, 3 \mathrm{H}) ;{ }^{13} \mathrm{C} \mathrm{NMR}\left(151 \mathrm{MHz}, d^{6}\right.$-DMSO) $\delta 174.3,142.1,139.3$, 135.4, 128.9, 128.4, 126.1, 123.2, 120.3, 50.7, 31.01, 22.7, 20.6 9.1. HRMS calcd. (ESI) $\mathrm{m} / \mathrm{z}$ for $\mathrm{C}_{18} \mathrm{H}_{21} \mathrm{NNaO}[\mathrm{M}+\mathrm{Na}]^{+}:$290.1515, found: 250.1513.

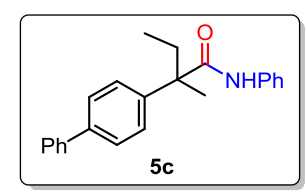

\section{2-([1,1'-biphenyl]-4-yl)-2-methyl- $N$-phenylbutanamide (5c)}

Yield $=60 \%, \mathrm{~b} / \mathrm{l}>99: 1,{ }^{1} \mathrm{H}$ NMR $\left(400 \mathrm{MHz}, \mathrm{CDCl}_{3}\right) \delta$ 7.63-7.60 (m, 4H), 7.48-7.43 (m, 4H), 7.40-7.34 (m, 3H), 7.28-7.24 (m, 2H), $7.05(\mathrm{t}, J=7.6 \mathrm{~Hz}, 1 \mathrm{H})$, $6.89(\mathrm{~s}, 1 \mathrm{H}), 2.24-2.09(\mathrm{~m}, 2 \mathrm{H}), 1.64(\mathrm{~s}, 3 \mathrm{H}), 0.88(\mathrm{t}, J=7.2 \mathrm{~Hz}, 3 \mathrm{H}),{ }^{13} \mathrm{C}$ NMR $(101$ $\left.\mathrm{MHz}, \mathrm{CDCl}_{3}\right) \delta 175.2,142.6,140.4,140.1,138.0,129.0,129.0,127.6,127.6,127.1$, 124.3, 119.8, 51.8, 31.7, 23.5, 8.9. HRMS calcd. (ESI) $\mathrm{m} / \mathrm{z}$ for $\mathrm{C}_{23} \mathrm{H}_{23} \mathrm{NNaO}[\mathrm{M}+\mathrm{Na}]^{+}$: 352.1671, found: 352.1659 . 


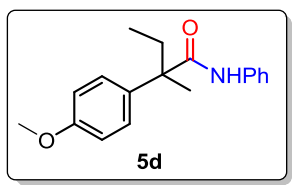

2-(4-methoxyphenyl)-2-methyl- $N$-phenylbutanamide (5d)

Yield $=74 \%, \mathrm{~b} / \mathrm{l}=87: 13,{ }^{1} \mathrm{H}$ NMR $\left(400 \mathrm{MHz}, \mathrm{CDCl}_{3}\right) \delta 7.37-7.35(\mathrm{~m}, 2 \mathrm{H})$, 7.33-7.30 (m, 2H), 7.27-7.23 (m, 2H), 7.06-7.02 (m, 1H), 6.94-6.91 (m, 2H), $6.86(\mathrm{~s}$, 1H), 3.278 (s, 3H), 1.92-1.86 (m, 1H), 2.18-2.04 (m, 2H), $1.58(\mathrm{~s}, 3 \mathrm{H}), 0.83$ (t, $J=7.2$ $\mathrm{Hz}, 3 \mathrm{H}) ;{ }^{13} \mathrm{C} \mathrm{NMR}\left(101 \mathrm{MHz}, \mathrm{CDCl}_{3}\right) \delta 175.7,158.7,138.1,135.3,128.9,128.3$, 124.1, 119.7, 114.3, 55.4, 51.3, 31.6, 23.5, 8.9. HRMS calcd. (ESI) $\mathrm{m} / \mathrm{z}$ for $\mathrm{C}_{18} \mathrm{H}_{21} \mathrm{NNaO}_{2}[\mathrm{M}+\mathrm{Na}]^{+}:$306.1464, found: 306.1464 .

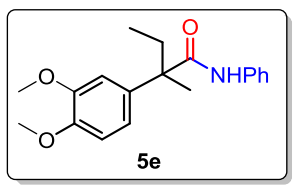

\section{2-(3,4-dimethoxyphenyl)-2-methyl- $N$-phenylbutanamide (5e)}

Yield $=76 \%, \mathrm{~b} / \mathrm{l}=88: 12,{ }^{1} \mathrm{H}$ NMR $\left(400 \mathrm{MHz}, \mathrm{CDCl}_{3}\right) \delta 7.36(\mathrm{~d}, J=7.6 \mathrm{~Hz}, 2 \mathrm{H})$, $7.25(\mathrm{t}, J=7.2 \mathrm{~Hz}, 2 \mathrm{H}), 7.04(\mathrm{t}, J=7.2 \mathrm{~Hz}, 1 \mathrm{H}), 6.97(\mathrm{dd}, J=7.4,2.0 \mathrm{~Hz}, 1 \mathrm{H}), 6.92$ (s, 1H), 6.89-6.86 (m, 2H), 3.90 (s, 3H), 3.85 (s, 3H), 2.20-2.04 (m, 2H), 1.59 (s, 3H), $0.83(\mathrm{t}, J=7.6 \mathrm{~Hz}, 3 \mathrm{H}) ;{ }^{13} \mathrm{C} \mathrm{NMR}\left(101 \mathrm{MHz}, \mathrm{CDCl}_{3}\right) \delta 175.6,149.2,148.2,138.0$, $135.8,128.9,124.1,119.6,119.0,111.0,110.3,56.0,55.9,51.5,31.5,23.3,8.9$. HRMS calcd. (ESI) $\mathrm{m} / \mathrm{z}$ for $\mathrm{C}_{19} \mathrm{H}_{23} \mathrm{NNaO}_{3}[\mathrm{M}+\mathrm{Na}]^{+}: 336.1570$, found: 336.1570 .

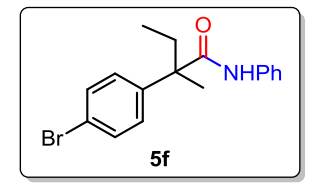

\section{2-(4-bromophenyl)-2-methyl- $N$-phenylbutanamide (5f)}

Yield $=51 \%, \mathrm{~b} / \mathrm{l}=96: 4,{ }^{1} \mathrm{H} \mathrm{NMR}\left(400 \mathrm{MHz}, \mathrm{CDCl}_{3}\right) \delta 7.51(\mathrm{~d}, J=8.8 \mathrm{~Hz}, 2 \mathrm{H})$, $7.37(\mathrm{~d}, J=8.0 \mathrm{~Hz}, 2 \mathrm{H}), 7.29-7.25(\mathrm{~m}, 4 \mathrm{H}), 7.07$ (t, $J=7.2 \mathrm{~Hz}, 1 \mathrm{H}), 6.78(\mathrm{~s}, 1 \mathrm{H})$, 2.17-2.00 (m, 2H), $1.58(\mathrm{~s}, 3 \mathrm{H}), 0.84(\mathrm{t}, J=7.6 \mathrm{~Hz}, 3 \mathrm{H}) ;{ }^{13} \mathrm{C} \mathrm{NMR}\left(101 \mathrm{MHz}, \mathrm{CDCl}_{3}\right)$ $\delta 174.5,142.8,137.9,132.1,129.1,128.9,124.4,121.4,119.9,51.7,31.7,23.3,8.9$. HRMS calcd. (ESI) $\mathrm{m} / \mathrm{z}$ for $\mathrm{C}_{17} \mathrm{H}_{18} \mathrm{BrNNaO} 3[\mathrm{M}+\mathrm{Na}]^{+}$: 354.0463 , found: 354.0462 . 


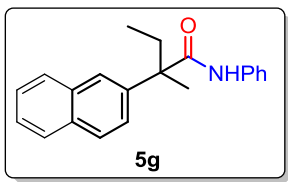

2-methyl-2-(naphthalen-2-yl)- $N$-phenylbutanamide (5g)

Yield $=57 \%, \mathrm{~b} / \mathrm{l}>99: 1,{ }^{1} \mathrm{H}$ NMR $\left(400 \mathrm{MHz}, \mathrm{CDCl}_{3}\right) \delta$ 7.88-7.83 (m, 4H), 7.54-7.44 (m, 3H), 7.34-7.32 (m, 2H), 7.24-7.20 (m, 2H), 7.05-7.01 (m, 1H), $6.83(\mathrm{~s}$, 1H), 2.30-2.20 (m, 2H), $1.70(\mathrm{~s}, 3 \mathrm{H}), 0.85(\mathrm{t}, J=7.6 \mathrm{~Hz}, 3 \mathrm{H}) ;{ }^{13} \mathrm{C}$ NMR $(101 \mathrm{MHz}$, $\left.\mathrm{CDCl}_{3}\right) \delta 175.2,141.0,138.0,133.4,132.5,129.0,128.9,128.2,127.7,126.6,126.4$, 125.7, 125.5, 124.2, 119.8, 52.1, 31.4, 23.3, 8.9. HRMS calcd. (ESI) $\mathrm{m} / \mathrm{z}$ for $\mathrm{C}_{21} \mathrm{H}_{21} \mathrm{NNaO}[\mathrm{M}+\mathrm{Na}]^{+}: 326.1515$, found: 326.1515 .

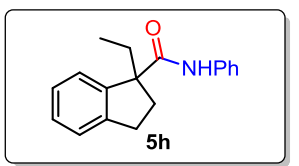

\section{1-ethyl- $N$-phenyl-2,3-dihydro-1H-indene-1-carboxamide $(5 \mathrm{~h})$}

Yield $=61 \%, \mathrm{~b} / \mathrm{l}=87: 13,{ }^{1} \mathrm{H}$ NMR $\left(400 \mathrm{MHz}, \mathrm{CDCl}_{3}\right) \delta$ 7.39-7.36 (m, 2H), 7.31-7.30 (m, 3H), 7.29-7.24 (m, 3H), $7.19(\mathrm{~s}, 1 \mathrm{H}), 7.06(\mathrm{t}, J=7.2 \mathrm{~Hz}, 1 \mathrm{H}), 3.04-2.91$ (m, 2H), 2.65-2.58 (m, 1H), 2.24-2.17 (m, 1H), 2.12-2.06 (m, 2H), $0.91(\mathrm{t}, J=7.6 \mathrm{~Hz}$, $3 \mathrm{H}) ;{ }^{13} \mathrm{C} \mathrm{NMR}\left(101 \mathrm{MHz}, \mathrm{CDCl}_{3}\right) \delta 174.3,145.6,144.1,139.0,129.0,128.3,127.2$, 125.7, 124.4, 124.3, 119.8, 61.4, 36.7, 30.5, 30.0, 9.6. HRMS calcd. (ESI) m/z for $\mathrm{C}_{18} \mathrm{H}_{19} \mathrm{NNaO}[\mathrm{M}+\mathrm{Na}]^{+}:$288.1358, found: 288.1358 .

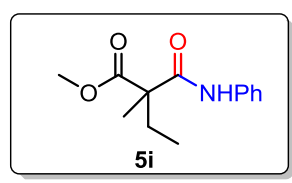

methyl 2-methyl-2-(phenylcarbamoyl)butanoate (5i)

Yield $=63 \%, \mathrm{~b} / \mathrm{l}=97: 3,{ }^{1} \mathrm{H}$ NMR $\left(400 \mathrm{MHz}, \mathrm{CDCl}_{3}\right) \delta 9.18(\mathrm{~s}, 1 \mathrm{H}), 7.57-7.55$ (m, 2H), 7.33 (t, $J=7.6 \mathrm{~Hz}, 2 \mathrm{H}), 7.11$ (t $J=7.6 \mathrm{~Hz}, 1 \mathrm{H}), 3.80(\mathrm{~s}, 3 \mathrm{H}), 2.15-2.60$ (m, 1H), 1.98-1.89 (m, 1H), $1.52(\mathrm{~s}, 3 \mathrm{H}), 0.91(\mathrm{t}, J=7.2 \mathrm{~Hz}, 3 \mathrm{H}) ;{ }^{13} \mathrm{C}$ NMR (101 MHz, $\left.\mathrm{CDCl}_{3}\right) \delta 176.4,169.3,137.9,129.1,124.4,120.1,54.9,53.0,32.2,20.6,9.7$. HRMS calcd. (ESI) m/z for $\mathrm{C}_{13} \mathrm{H}_{17} \mathrm{NNaO}_{3}[\mathrm{M}+\mathrm{Na}]^{+}:$258.1100, found:258.1100. 


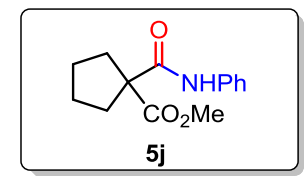

methyl 1-(phenylcarbamoyl)cyclopentane-1-carboxylate (5j)

Yield $=65 \%, \mathrm{~b} / \mathrm{l}=86: 14,{ }^{1} \mathrm{H}$ NMR $\left(400 \mathrm{MHz}, \mathrm{CDCl}_{3}\right) \delta 8.23(\mathrm{~s}, 1 \mathrm{H}), 7.53-7.51$ (m, 2H), $7.32(\mathrm{t}, J=7.6 \mathrm{~Hz}, 2 \mathrm{H}), 7.10(\mathrm{t}, J=7.2 \mathrm{~Hz}, 1 \mathrm{H}), 3.78(\mathrm{~s}, 3 \mathrm{H}), 2.33-2.27(\mathrm{~m}$, 4H), 1.81-1.67 (m, 4H); ${ }^{13} \mathrm{C}$ NMR (101 MHz, $\left.\mathrm{CDCl}_{3}\right) \delta 175.7,169.0,138.0,129.1$, 124.4, 119.9, 61.8, 53.2, 35.1, 25.5, 19.3. HRMS calcd. (ESI) $\mathrm{m} / \mathrm{z}$ for $\mathrm{C}_{14} \mathrm{H}_{17} \mathrm{NNaO}_{3}$ $[\mathrm{M}+\mathrm{Na}]^{+}: 270.1100$, found: 270.1100 .

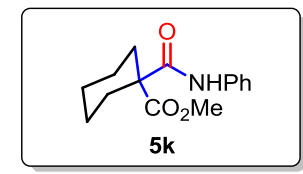

methyl 1-(phenylcarbamoyl)cyclohexane-1-carboxylate (5k)

Yield $=61 \%, \mathrm{~b} / \mathrm{l}=89: 11,{ }^{1} \mathrm{H}$ NMR $\left(400 \mathrm{MHz}, \mathrm{CDCl}_{3}\right) \delta 7.79(\mathrm{~s}, 1 \mathrm{H}), 7.52-7.49$ (m, 2H), $7.31(\mathrm{t}, J=7.6 \mathrm{~Hz}, 2 \mathrm{H}), 7.10(\mathrm{t}, J=7.2 \mathrm{~Hz}, 1 \mathrm{H}), 3.79(\mathrm{~s}, 3 \mathrm{H}), 2.28-2.24(\mathrm{~m}$, 2H) $1.96-1.89(\mathrm{~m}, 2 \mathrm{H}), 1.76-1.60(\mathrm{~m}, 2 \mathrm{H}), 1.54-1.45(\mathrm{~m}, 2 \mathrm{H}), 1.38-1.32(\mathrm{~m}, 2 \mathrm{H}) ;{ }^{13} \mathrm{C}$ NMR (101 MHz, $\left.\mathrm{CDCl}_{3}\right) \delta 174.4,168.6,137.8,129.1,124.6,119.9,56.2,53.0,32.1$, 25.3, 23.4. HRMS calcd. (ESI) $\mathrm{m} / \mathrm{z}$ for $\mathrm{C}_{15} \mathrm{H}_{19} \mathrm{NNaO}[\mathrm{M}+\mathrm{Na}]^{+}$: 284.1257, found: 284.1257.

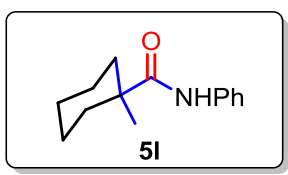

\section{1-methyl- $N$-phenylcyclohexane-1-carboxamide (5l)}

Yield $=55 \%, \mathrm{~b} / \mathrm{l}=80: 20,{ }^{1} \mathrm{H} \mathrm{NMR}\left(600 \mathrm{MHz}, \mathrm{CDCl}_{3}\right) \delta 7.54(\mathrm{~d}, J=7.2 \mathrm{~Hz}, 2 \mathrm{H})$, $7.37(\mathrm{~s}, 1 \mathrm{H}), 7.33-7.30(\mathrm{~m}, 2 \mathrm{H}), 7.10(\mathrm{t}, J=7.2 \mathrm{~Hz}, 1 \mathrm{H}), 2.03-2.01(\mathrm{~m}, 2 \mathrm{H}), 1.62-1.58$ $(\mathrm{m}, 2 \mathrm{H}), 1.54-1.47(\mathrm{~m}, 3 \mathrm{H}), 1.45-1.41(\mathrm{~m}, 2 \mathrm{H}), 1.39-1.36(\mathrm{~m}, 1 \mathrm{H}), 1.26(\mathrm{~s}, 3 \mathrm{H}) ;{ }^{13} \mathrm{C}$ NMR (101 MHz, $\left.\mathrm{CDCl}_{3}\right) \delta 174.0,138.2,129.1,124.2,120.1,43.7,35.8,26.6,25.9$, 23.0. HRMS calcd. (ESI) $\mathrm{m} / \mathrm{z}$ for $\mathrm{C}_{14} \mathrm{H}_{19} \mathrm{NNaO}$ [M+Na] ${ }^{+}$: 240.1358, found: 240.1357 . 


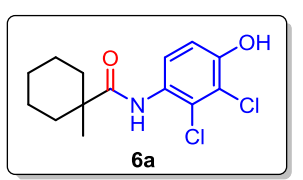

$N$-(2,3-dichloro-4-hydroxyphenyl)-1-methylcyclohexane-1-carboxamide (6a)

Yield $=62 \%, \mathrm{~b} / \mathrm{l}=95: 5,{ }^{1} \mathrm{H} \mathrm{NMR}\left(400 \mathrm{MHz}, \mathrm{CDCl}_{3}\right) \delta 8.05(\mathrm{~d}, J=9.2 \mathrm{~Hz}, 1 \mathrm{H})$, $7.75(\mathrm{~s}, 1 \mathrm{H}), 6.94(\mathrm{~d}, J=9.2 \mathrm{~Hz}, 1 \mathrm{H}), 5.93(\mathrm{~s}, 1 \mathrm{H}), 2.06-2.02(\mathrm{~m}, 2 \mathrm{H}), 1.65-1.60(\mathrm{~m}$, 2H), 1.57-1.52 (m, 4H), 1.46-1.37 (m, 2H), 1.29 (s, 3H); $\left.{ }^{13} \mathrm{C} \mathrm{NMR} \mathrm{(151} \mathrm{MHz,} \mathrm{CDCl}_{3}\right)$ $\delta 176.5,149.4,128.9,123.3,122.1,119.1,114.8,44.2,35.8,26.7,25.8,23.0$. HRMS calcd. (ESI) $\mathrm{m} / \mathrm{z}$ for $\mathrm{C}_{14} \mathrm{H}_{17} \mathrm{Cl}_{2} \mathrm{NNaO}_{2}[\mathrm{M}+\mathrm{Na}]^{+}$: 324.0528 , found: 324.0529 .

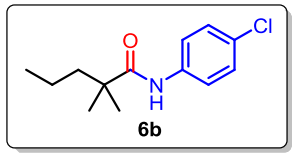

\section{$N$-(4-chlorophenyl)-2,2-dimethylpentanamide (6b)}

Yield $=71 \%, \mathrm{~b} / \mathrm{l}=95: 5,{ }^{1} \mathrm{H}$ NMR $\left(400 \mathrm{MHz}, \mathrm{CDCl}_{3}\right) \delta$ 7.47-7.43 (m, 3H), 7.27-7.23 (m, 2H), 1.58-1.54 (m, 2H), 1.35-1.26 (m, 8H), $0.90(\mathrm{t}, J=7.2 \mathrm{~Hz}, 3 \mathrm{H}) ;{ }^{13} \mathrm{C}$ NMR $\left(101 \mathrm{MHz}, \mathrm{CDCl}_{3}\right) \delta 176.3,136.7,129.2,128.9,121.6,43.9,43.2,25.5,18.2$, 14.7. HRMS calcd. (ESI) $\mathrm{m} / \mathrm{z}$ for $\mathrm{C}_{13} \mathrm{H}_{18} \mathrm{ClNNaO}[\mathrm{M}+\mathrm{Na}]^{+}: 262.0969$, found: 262.0969 .

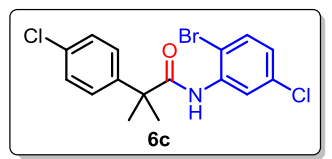

\section{$N$-(2-bromo-5-chlorophenyl)-2-(4-chlorophenyl)-2-methylpropanamide (6c)}

Yield $=62 \%, \mathrm{~b} / \mathrm{l}=97: 3,{ }^{1} \mathrm{H}$ NMR $\left(400 \mathrm{MHz}, \mathrm{CDCl}_{3}\right) \delta 8.43(\mathrm{~d}, J=2.4 \mathrm{~Hz}, 1 \mathrm{H})$, $7.47(\mathrm{~s}, 1 \mathrm{H}), 7.42-7.37(\mathrm{~m}, 4 \mathrm{H}), 7.33(\mathrm{~d}, J=8.4 \mathrm{~Hz}, 1 \mathrm{H}), 6.90(\mathrm{dd}, J=8.6,2.4 \mathrm{~Hz}$, 1H), $1.68(\mathrm{~s}, 6 \mathrm{H}) ;{ }^{13} \mathrm{C}$ NMR $\left(101 \mathrm{MHz}, \mathrm{CDCl}_{3}\right) \delta 175.2,142.4,136.5,134.3,133.7$, 132.7, 129.3, 128.1, 125.0, 121.2, 111.0, 48.2, 26.8. HRMS calcd. (ESI) m/z for $\mathrm{C}_{16} \mathrm{H}_{15} \mathrm{BrClNNaO}[\mathrm{M}+\mathrm{Na}]^{+}:$409.9504, found: 409.9514 .

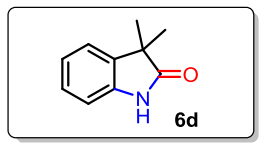

\section{3,3-dimethylindolin-2-one (6d)}

Yield $=95 \%, \mathrm{~b} / \mathrm{l}>99: 1 ;{ }^{1} \mathrm{H}$ NMR $\left(400 \mathrm{MHz}, \mathrm{CDCl}_{3}\right) \delta 8.81(\mathrm{~s}, 1 \mathrm{H}), 7.22-7.21$ $(\mathrm{m}, 2 \mathrm{H}), 7.06-7.02(\mathrm{~m}, 1 \mathrm{H}), 6.96-6.93(\mathrm{~m}, 1 \mathrm{H}), 1.41(\mathrm{~s}, 6 \mathrm{H}) ;{ }^{13} \mathrm{C}$ NMR $(101 \mathrm{MHz}$, $\left.\mathrm{CDCl}_{3}\right) \delta 184.3,140.0,136.1,127.8,122.7,122.6,110.0,44.8,24.5$. HRMS calcd. (ESI) $\mathrm{m} / \mathrm{z}$ for $\mathrm{C}_{10} \mathrm{H}_{11} \mathrm{NNaO}[\mathrm{M}+\mathrm{Na}]^{+}$: 184.0732, found: 184.0728 . 
7. X-Ray Structure of 3qa.

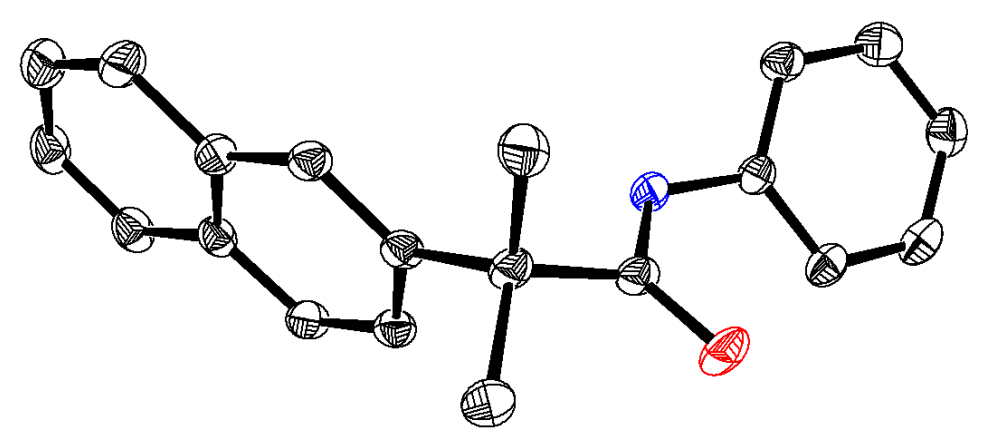

\begin{tabular}{|l|l|}
\hline \multirow{2}{*}{ Bond precision } & C-C $=0.0021 \mathrm{~A}$ \\
\cline { 2 - 2 } & Wavelength $=1.34139$ \\
\hline \multirow{3}{*}{ Cell } & $\mathrm{a}=9.145(3) \quad \alpha=90$ \\
\cline { 2 - 2 } & $\mathrm{b}=18.331(6) \quad$ $\quad$ ( $=110.206(12)$ \\
\cline { 2 - 2 } Temperature & $\mathrm{c}=10.162(3) \quad \gamma=90$ \\
\hline Volume & $150 \mathrm{~K}$ \\
\hline Space group & $1598.7(9)$ \\
\hline Sum formula & $\mathrm{P} 21 / \mathrm{c}$ \\
\hline Mr & $\mathrm{C} 20 \mathrm{H} 19 \mathrm{~N} \mathrm{O}$ \\
\hline Dx, g cm ${ }^{-3}$ & 289.36 \\
\hline Z & 1.202 \\
\hline Mu (mm-1) & 4 \\
\hline F000 & 0.364 \\
\hline h,k,lmax & 616.0 \\
\hline Nref & $11,22,12$ \\
\hline Tmin,Tmax & 3032 \\
\hline Correction method= \# Reported T Limits & Not given \\
\hline AbsCorr $=$ MULTT-SCAN & \\
\hline Data completeness & 0.985 \\
\hline Theta(max) & 54.802 \\
\hline R(reflections) & $0.0539(2495)$ \\
\hline wR2(reflections) & $0.1982(2988)$ \\
\hline S & 0.864 \\
\hline Npar & 201 \\
\hline
\end{tabular}




\section{Copies of ${ }^{1} \mathrm{H},{ }^{13} \mathrm{C}$, and ${ }^{19} \mathrm{~F}$ NMR Spectra}

${ }^{1} \mathrm{H}$ NMR Spectra $\left(400 \mathrm{MHz}, \mathrm{CDCl}_{3}\right)$ of compound 3aa

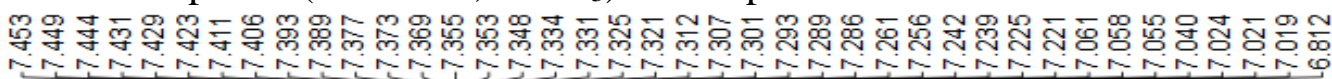
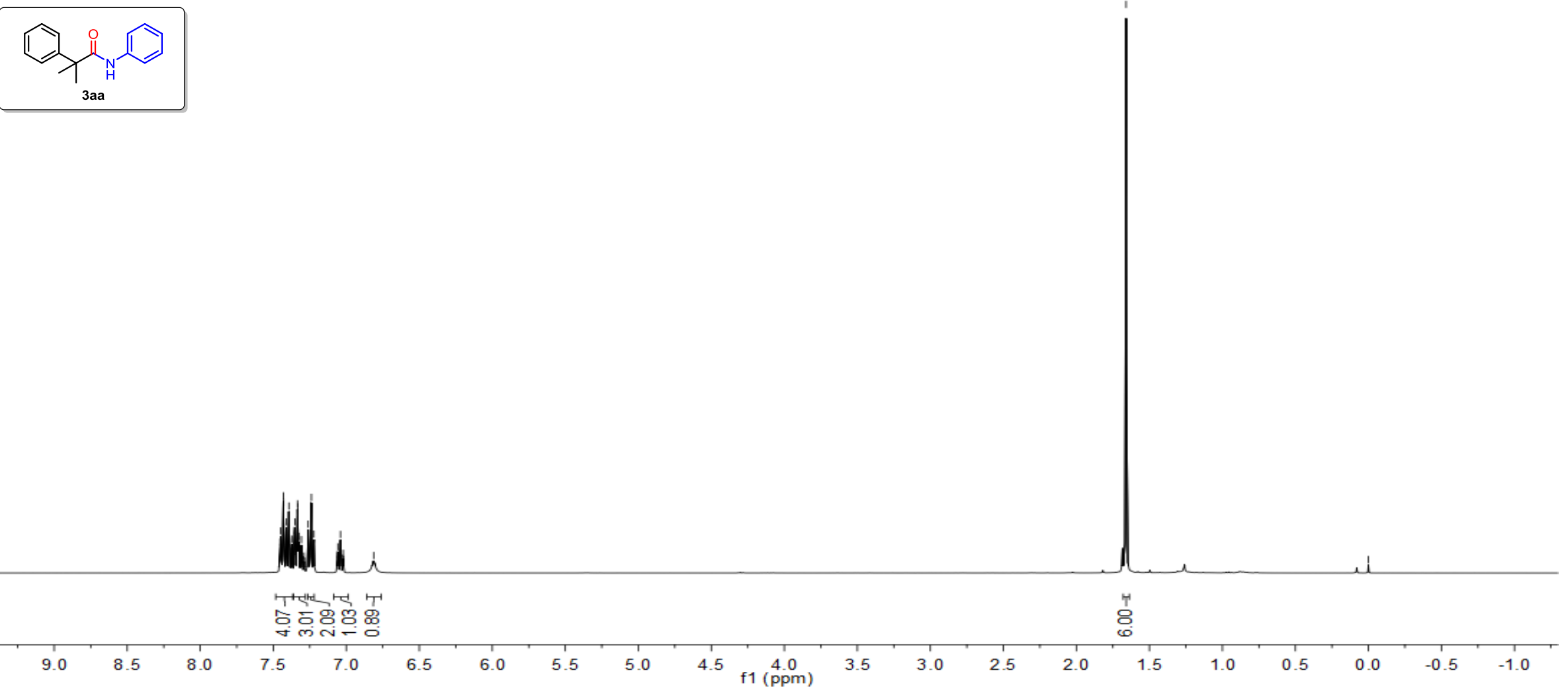
${ }^{13} \mathrm{C}$ NMR Spectra (101 MHz, $\mathrm{CDCl}_{3}$ ) of compound 3aa
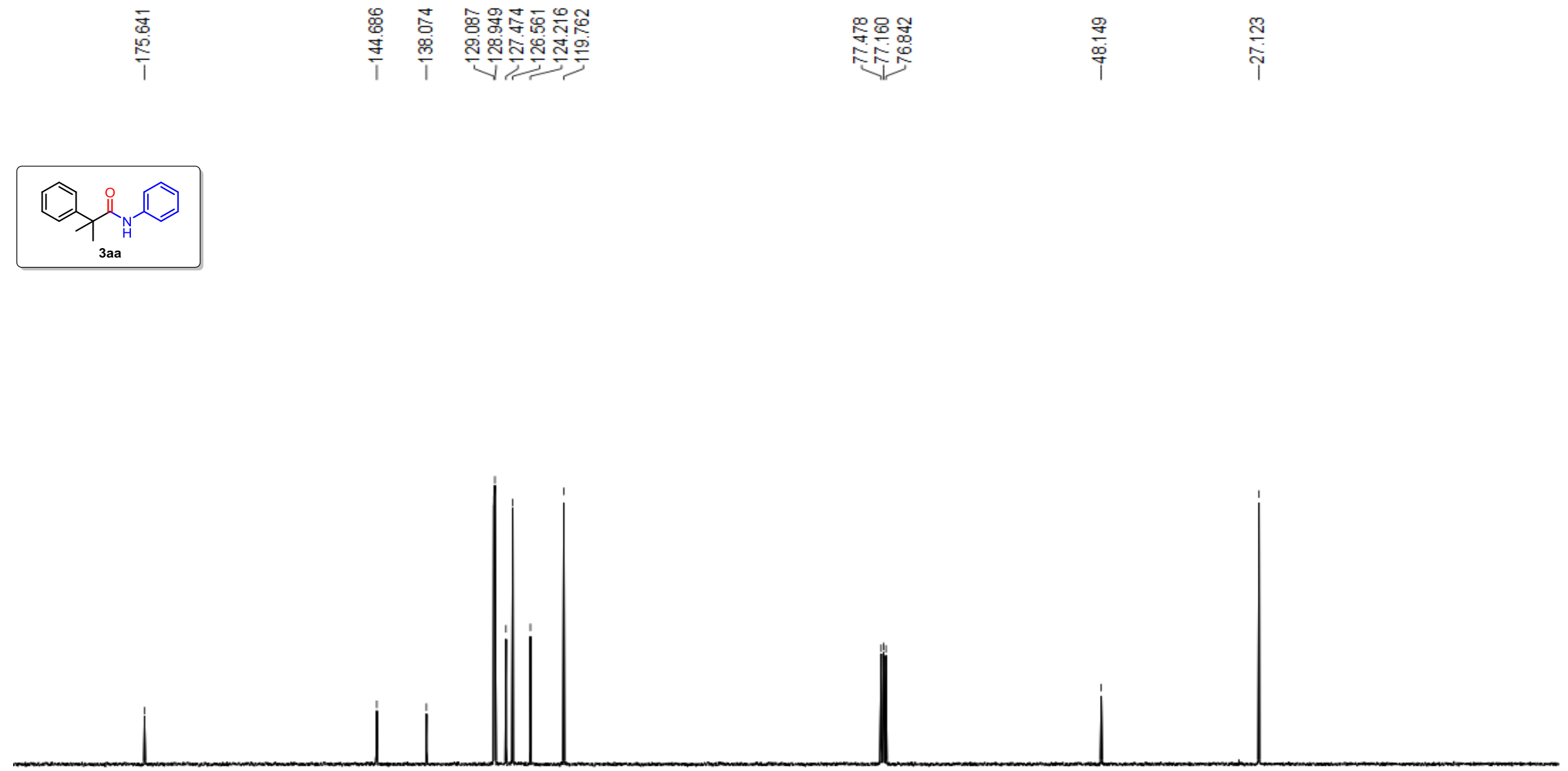
${ }^{1} \mathrm{H}$ NMR Spectra (400 MHz, $\mathrm{CDCl}_{3}$ ) of compound 3ab

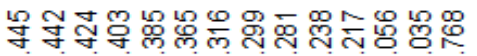

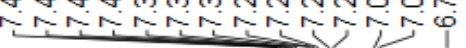
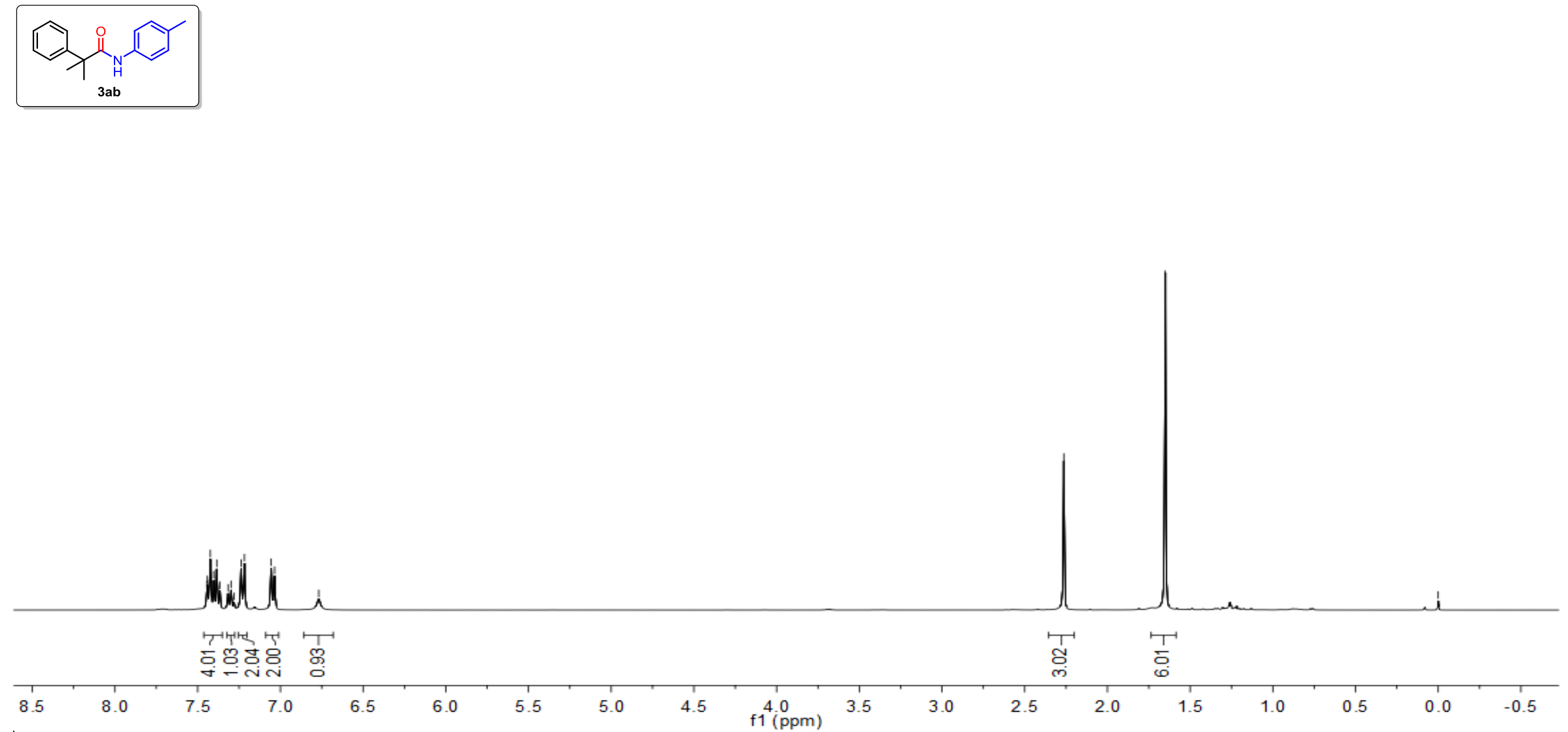
${ }^{13} \mathrm{C}$ NMR Spectra (101 MHz, $\mathrm{CDCl}_{3}$ ) of compound 3ab
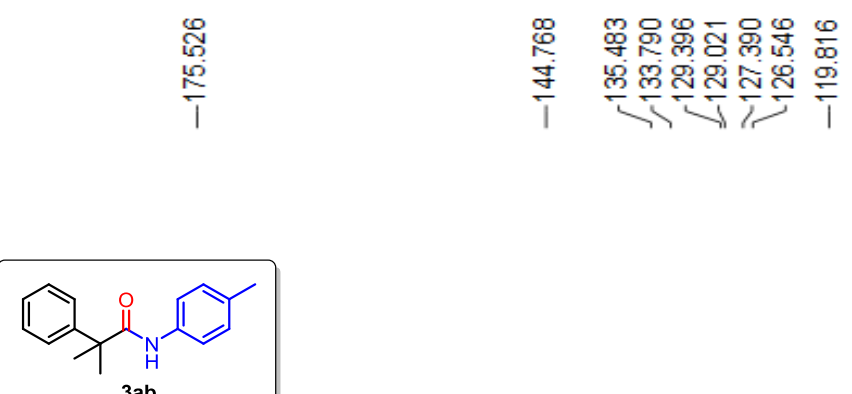

$3 a b$

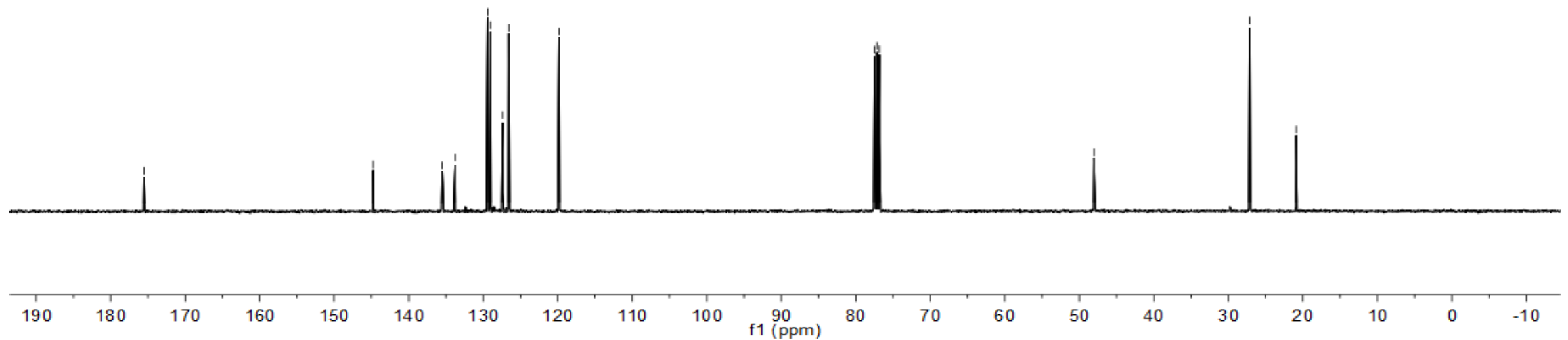


${ }^{1} \mathrm{H}$ NMR Spectra (400 MHz, $\mathrm{CDCl}_{3}$ ) of compound 3ac

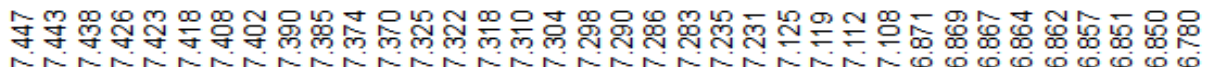

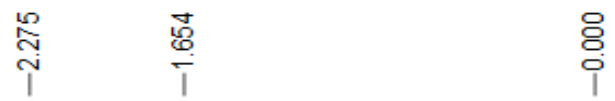

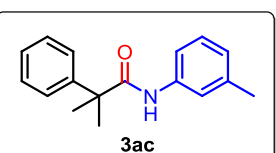

$3 a c$

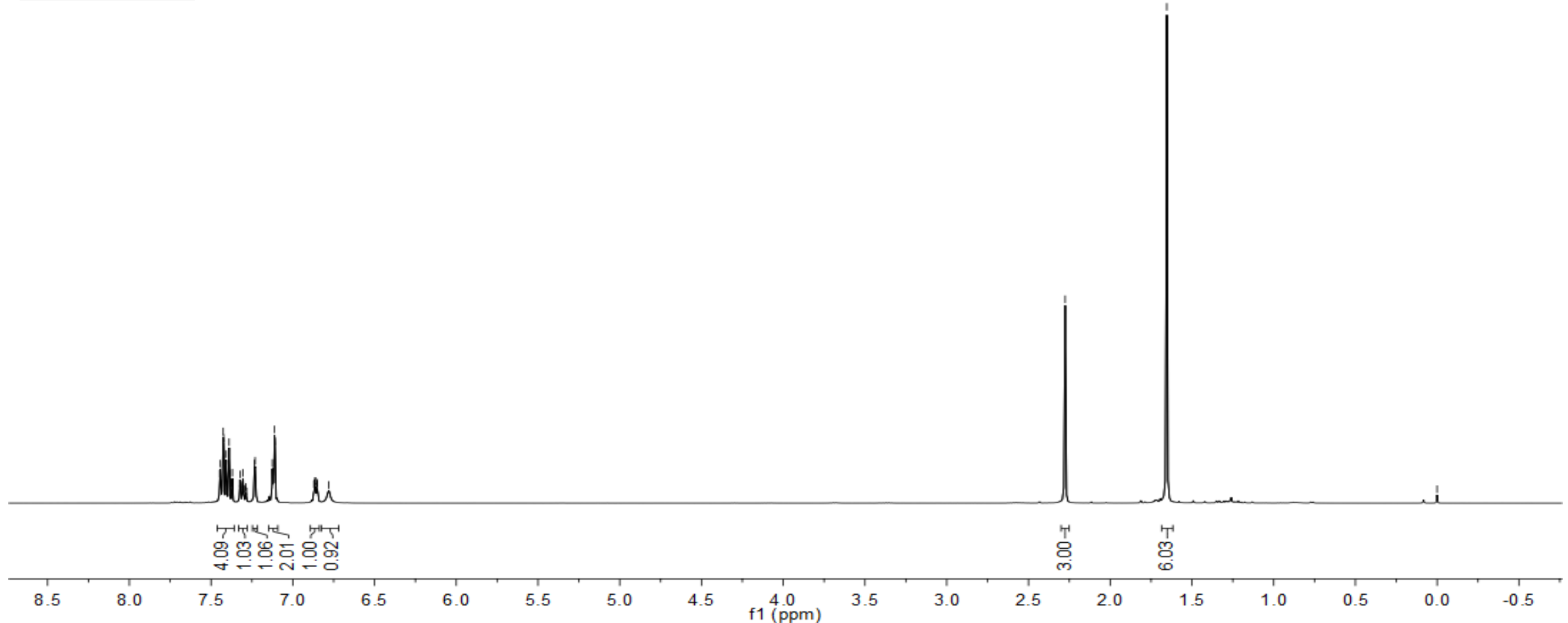


${ }^{13} \mathrm{C}$ NMR Spectra (101 MHz, $\mathrm{CDCl}_{3}$ ) of compound 3a

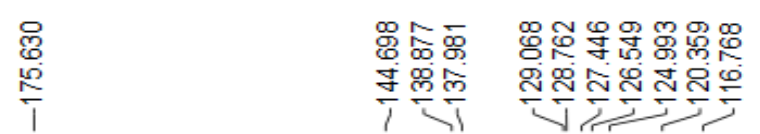

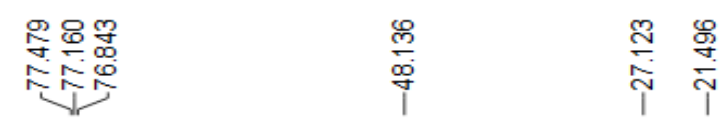
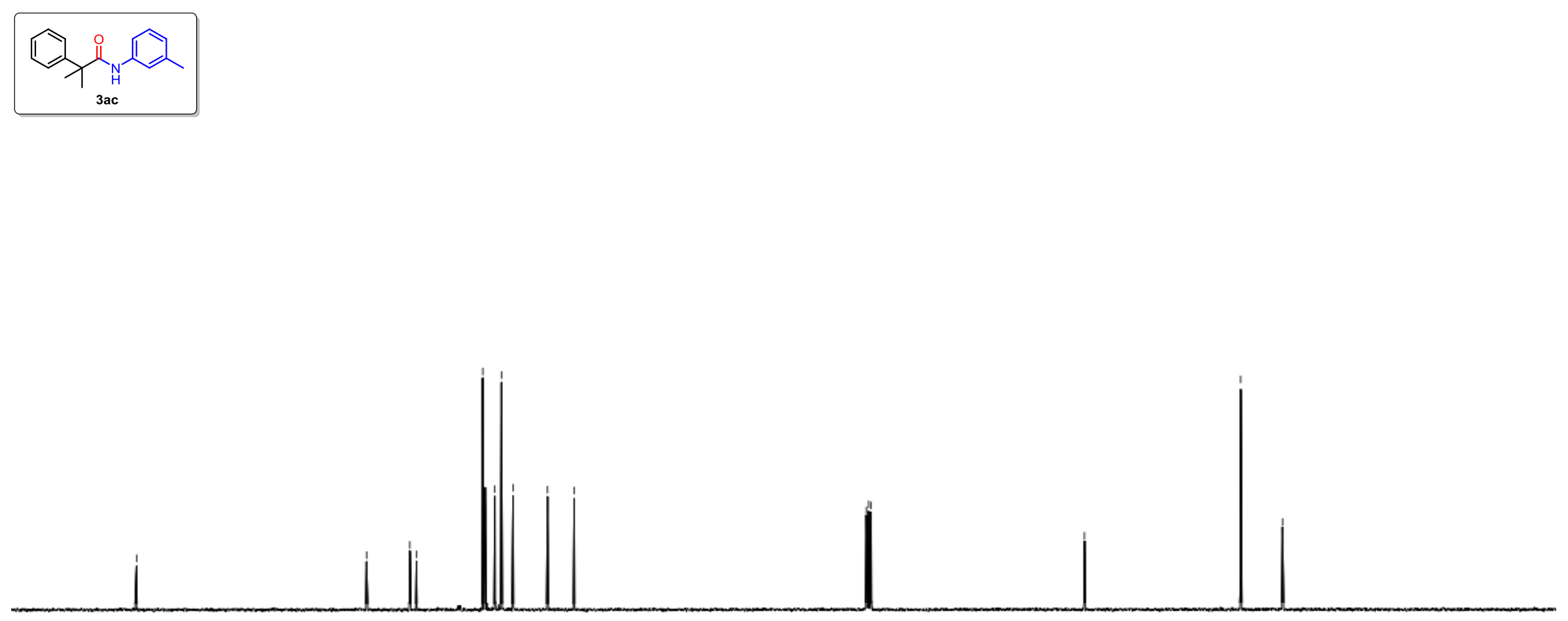

190

180

$170 \quad 160$

150

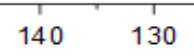

120

$110 \quad 100$

90

70

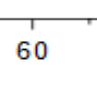

50 


\section{${ }^{1} \mathrm{H}$ NMR Spectra (400 MHz, $\mathrm{CDCl}_{3}$ ) of compound 3ad}

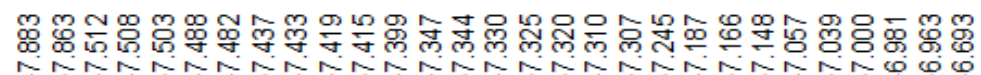

-
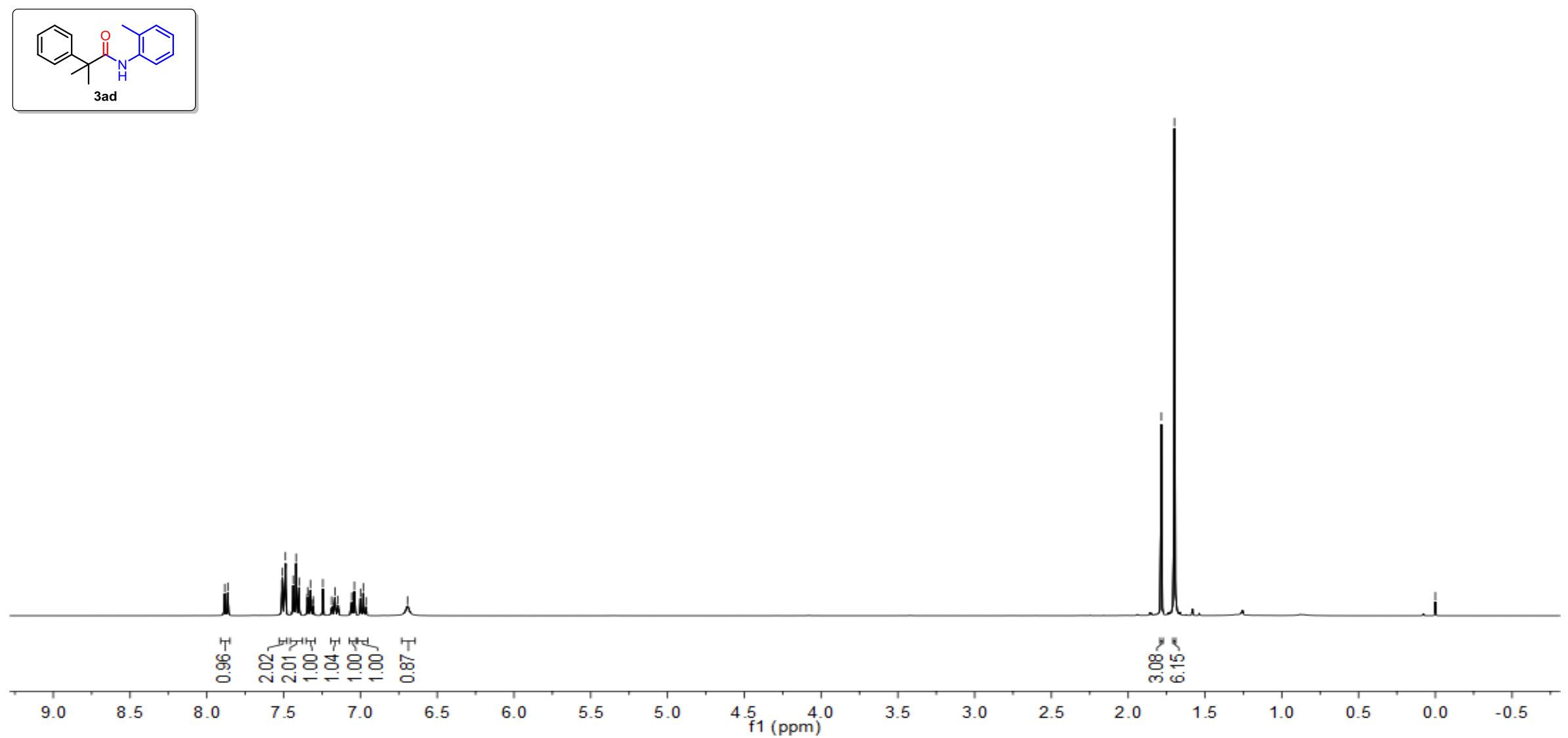
${ }^{13} \mathrm{C}$ NMR Spectra (101 MHz, $\mathrm{CDCl}_{3}$ ) of compound 3ad

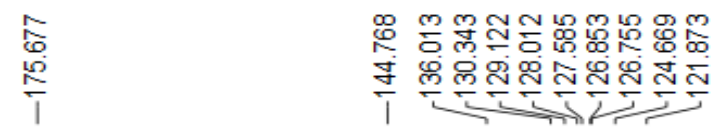

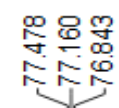

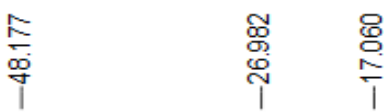

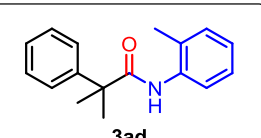

3 ad

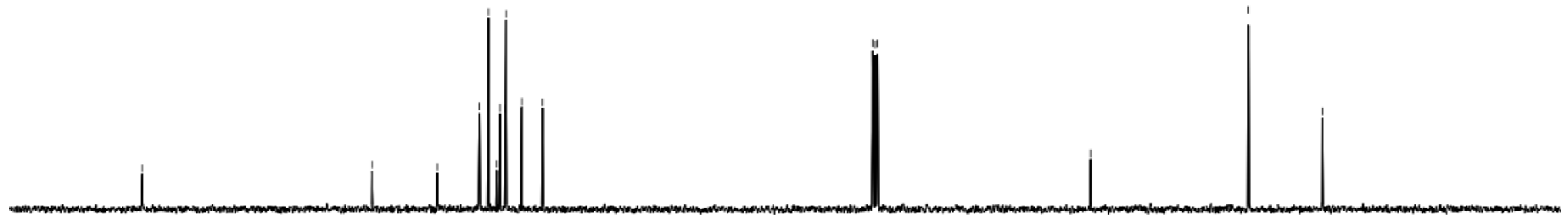

90

70
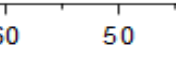
${ }^{1} \mathrm{H}$ NMR Spectra (400 MHz, $\mathrm{CDCl}_{3}$ ) of compound 3ae

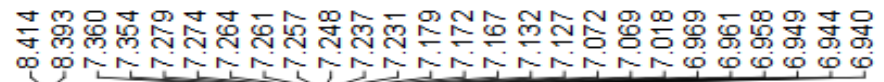
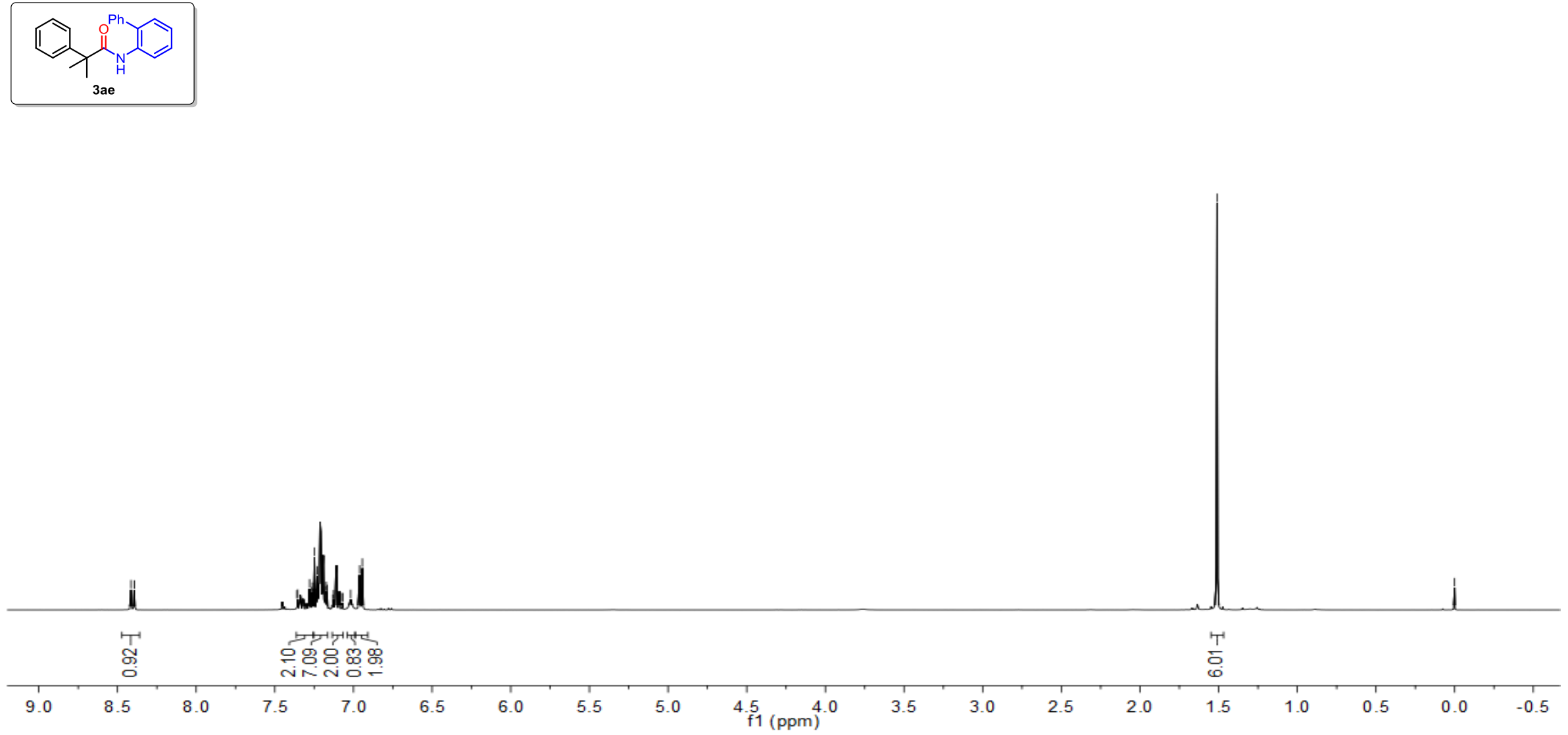
${ }^{13} \mathrm{C}$ NMR Spectra (101 MHz, $\mathrm{CDCl}_{3}$ ) of compound 3ae

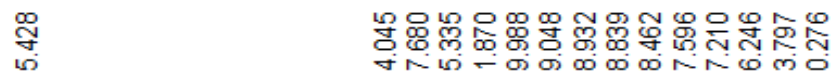

1 $\quad$ 눈

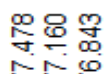

致

$\underset{\substack{8 \\ \text { o } \\ \text { o }}}{0}$

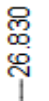
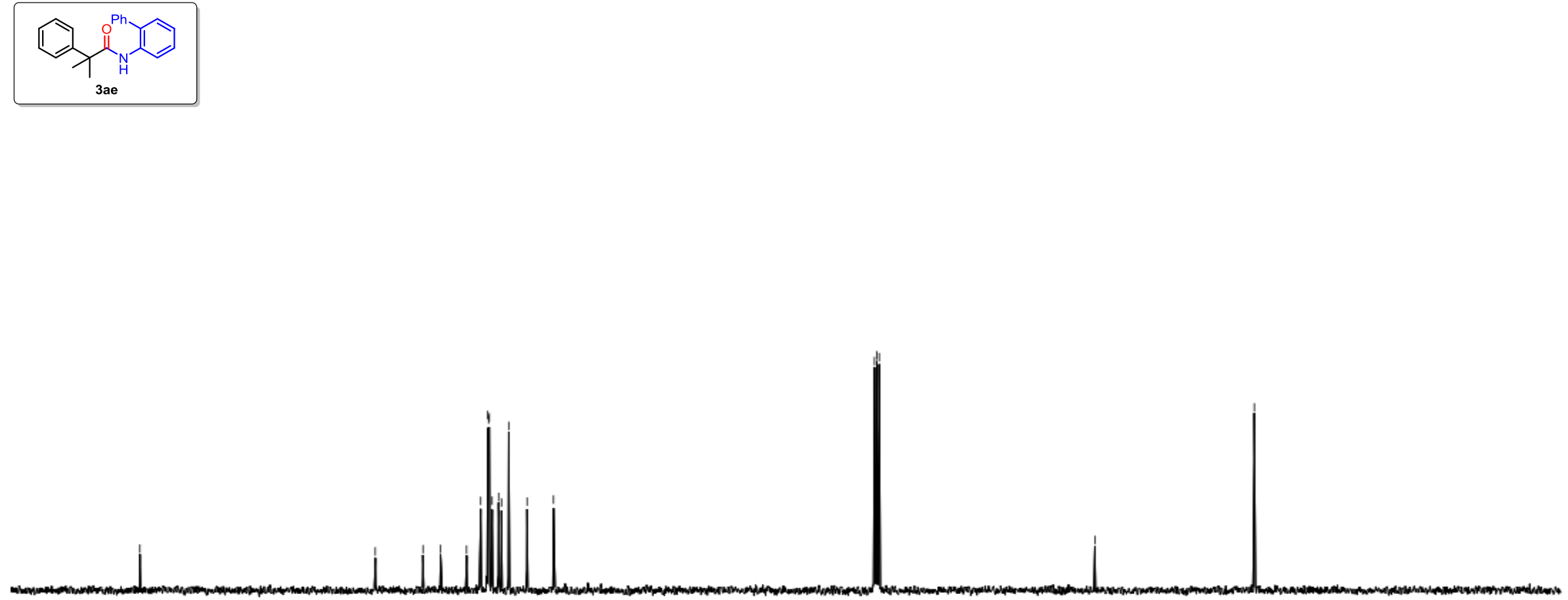

190

180

170

$160 \quad 150$

140

130

120

110

100

$90 \quad 80$

70

60

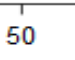

40

30

20 
${ }^{1} \mathrm{H}$ NMR Spectra (400 MHz, $\mathrm{CDCl}_{3}$ ) of compound 3af

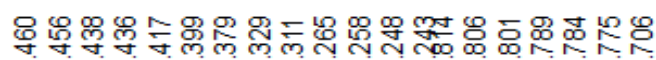

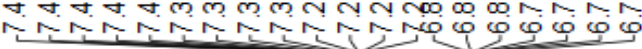
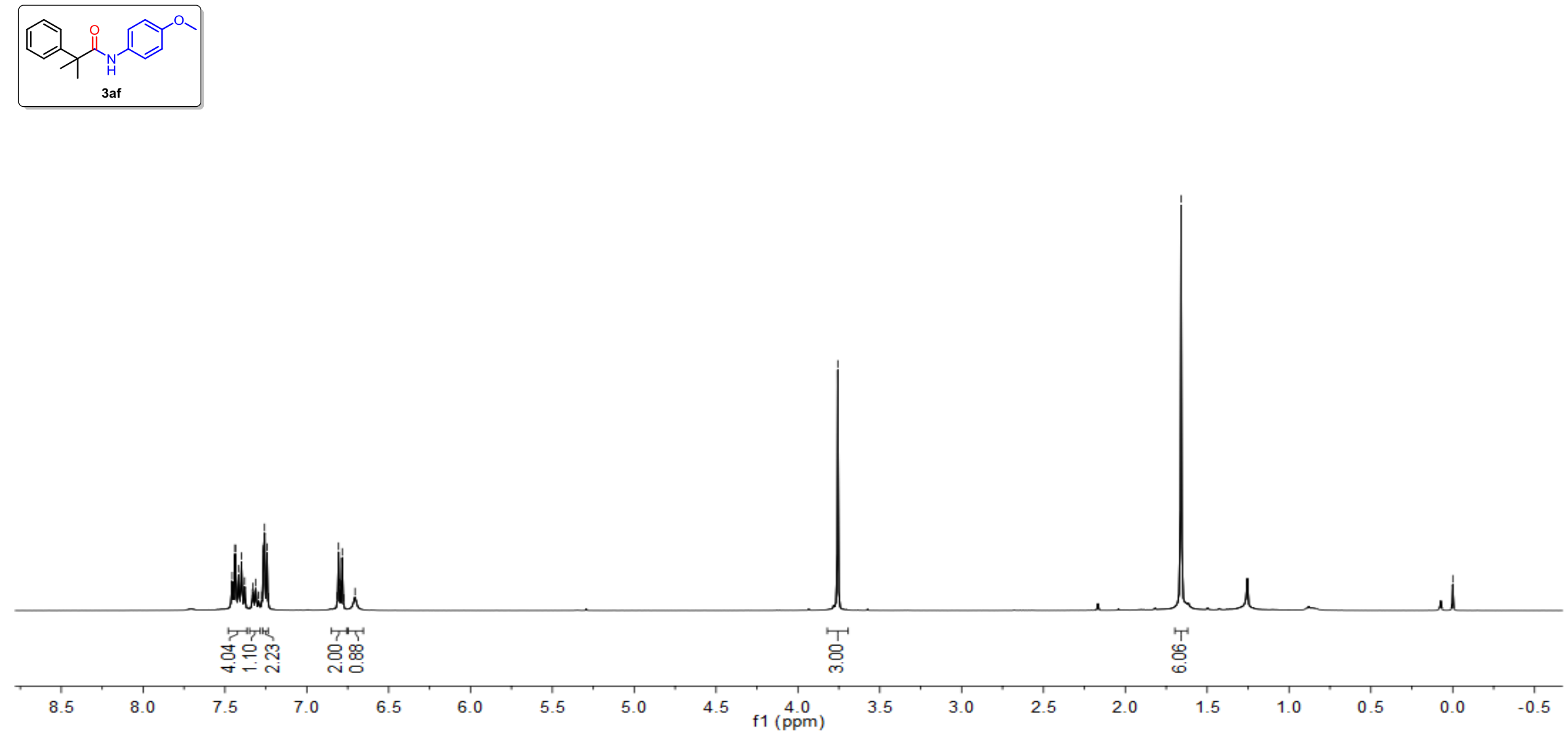
${ }^{13} \mathrm{C}$ NMR Spectra (101 MHz, $\mathrm{CDCl}_{3}$ ) of compound 3af

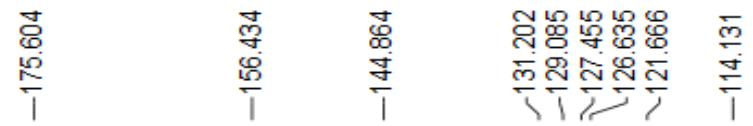

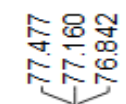

造

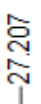
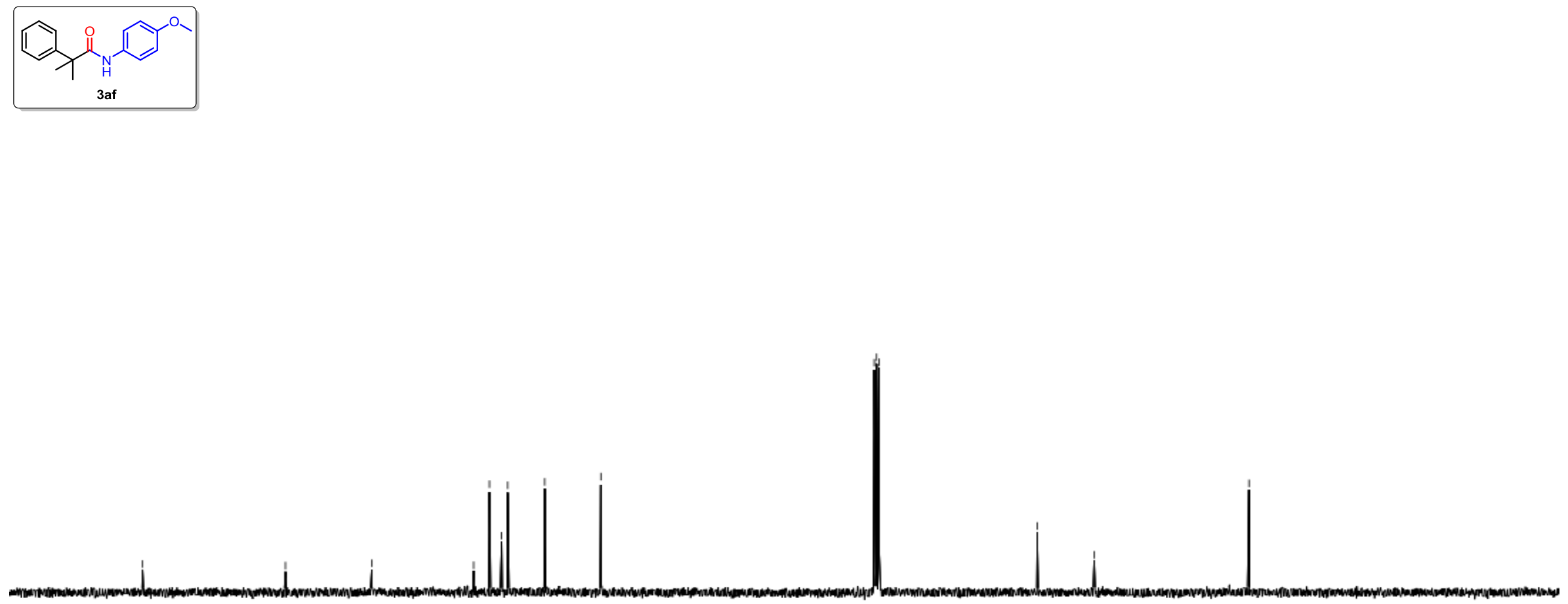

$190 \quad 180$

170

160

150

$140 \quad 130$

120

110

100

f1 $\stackrel{90}{(\mathrm{ppm})}$

80

70

60
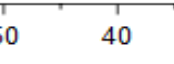

30 
${ }^{1} \mathrm{H}$ NMR Spectra (400 MHz, $\mathrm{CDCl}_{3}$ ) of compound $\mathbf{3 a g}$

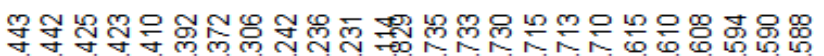

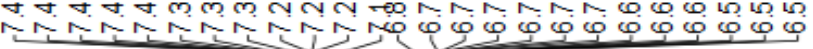

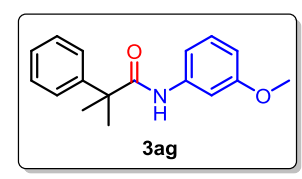

3ag

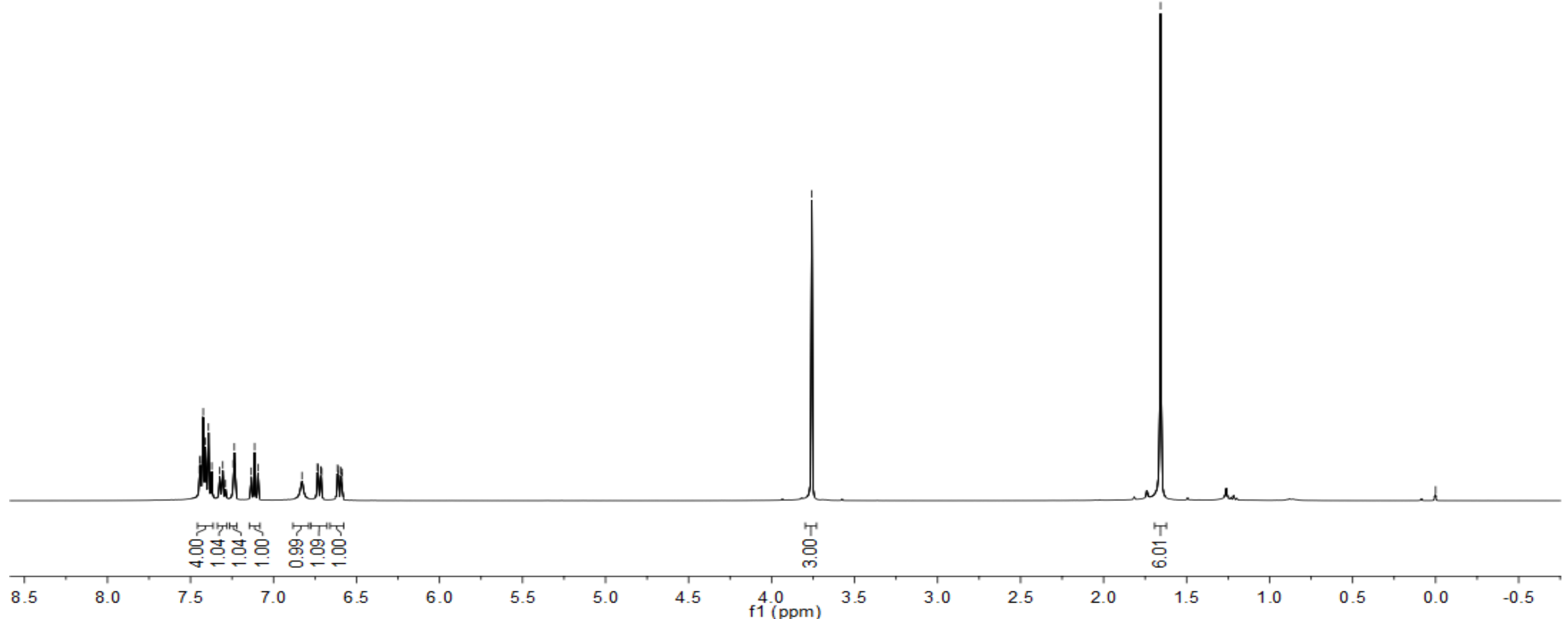


${ }^{13} \mathrm{C}$ NMR Spectra (101 MHz, $\mathrm{CDCl}_{3}$ ) of compound 3ag

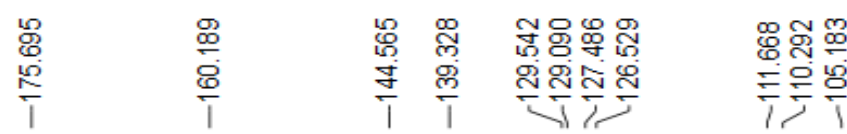

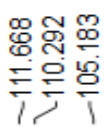
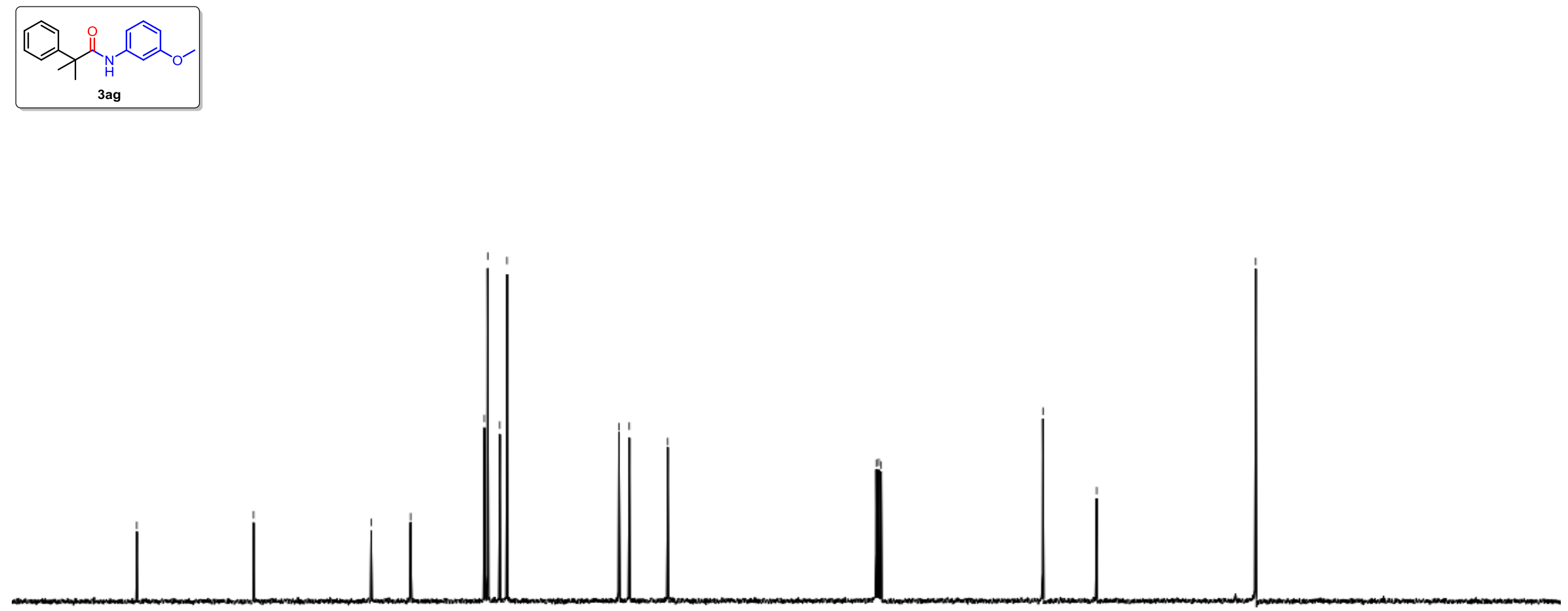
${ }^{1} \mathrm{H}$ NMR Spectra (400 MHz, $\mathrm{CDCl}_{3}$ ) of compound 3ah

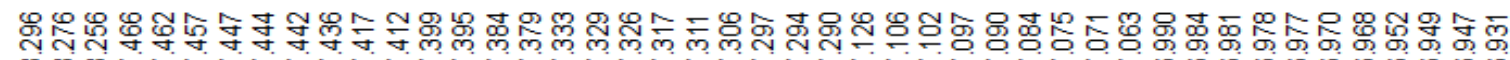

o oo

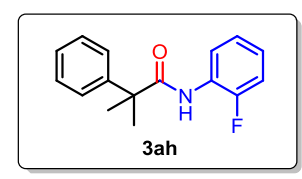

3ah

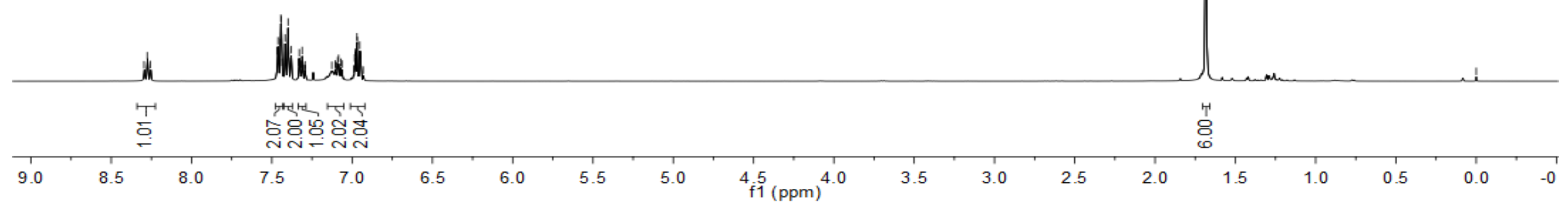


${ }^{13} \mathrm{C}$ NMR Spectra (101 MHz, $\mathrm{CDCl}_{3}$ ) of compound 3ah
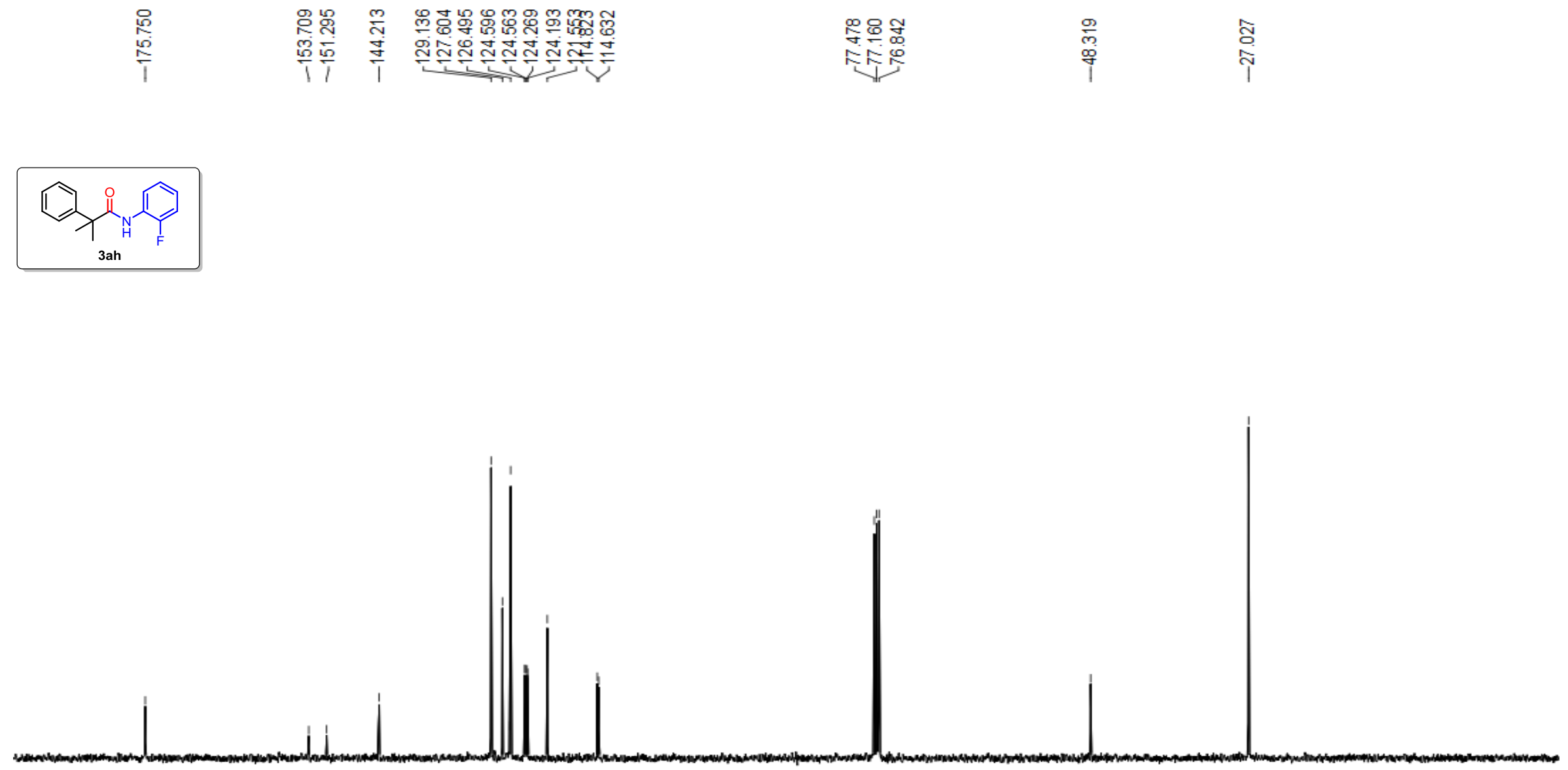
${ }^{19} \mathrm{~F}$ NMR Spectra (375 MHz, $\mathrm{CDCl}_{3}$ ) of compound 3ah

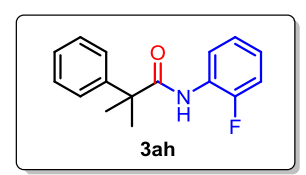

$\begin{array}{lllll} & 1 & 1 & 1 & \\ 80 & 70 & 60 & 50 & 40\end{array}$
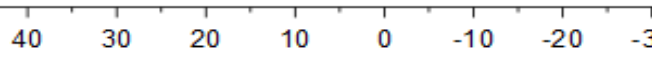

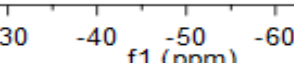

$\begin{array}{lllll}-50 & -60 & -70 & -80 & -90\end{array}$

$-110$

$-130$

$-150$ 
${ }^{1} \mathrm{H}$ NMR Spectra (400 MHz, $\mathrm{CDCl}_{3}$ ) of compound 3ah

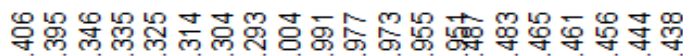

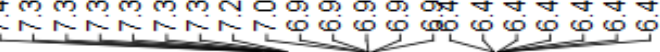
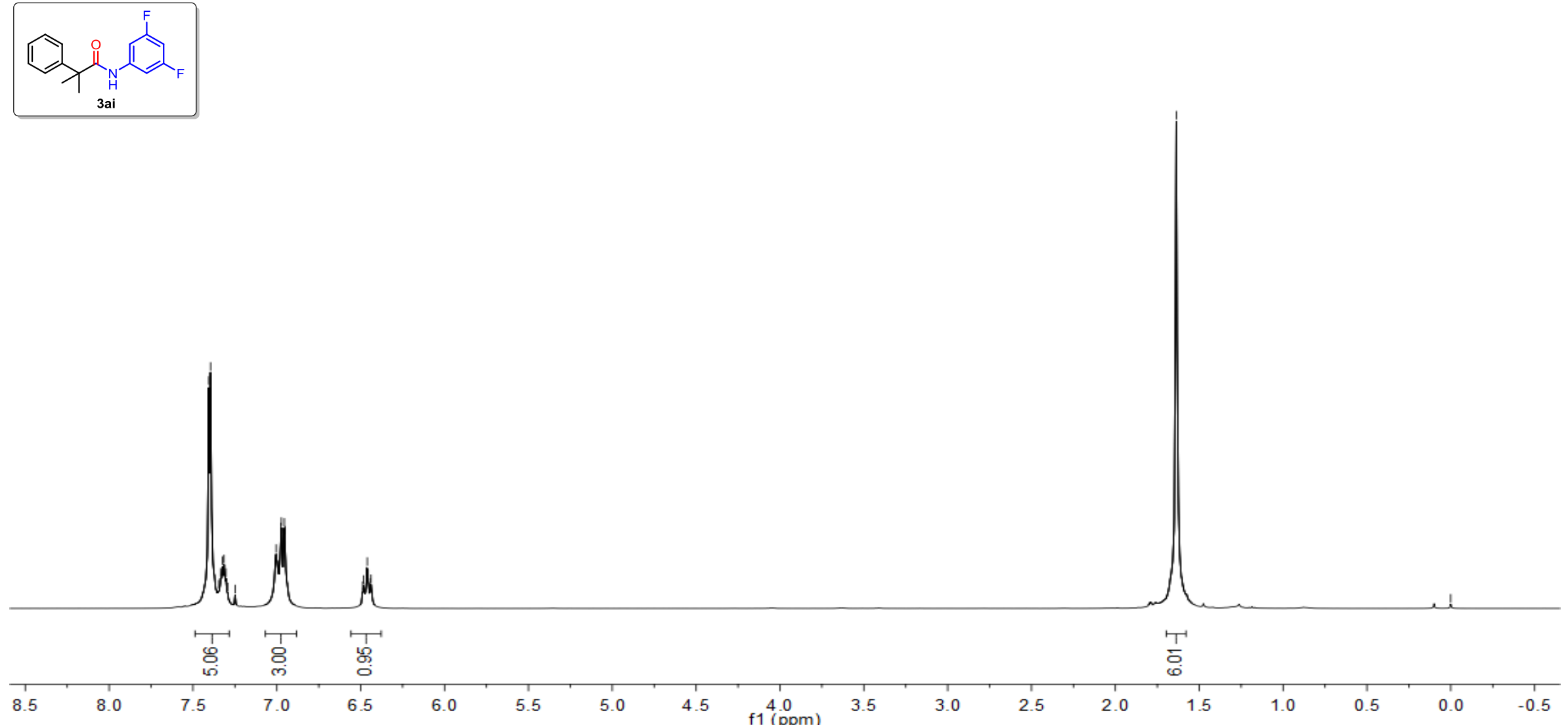
${ }^{13} \mathrm{C}$ NMR Spectra (101 MHz, $\mathrm{CDCl}_{3}$ ) of compound 3ai

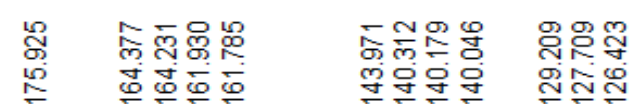

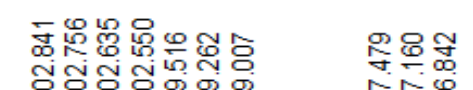

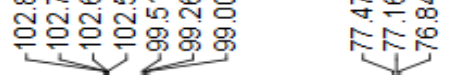

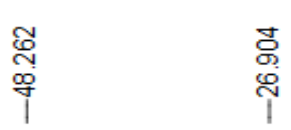
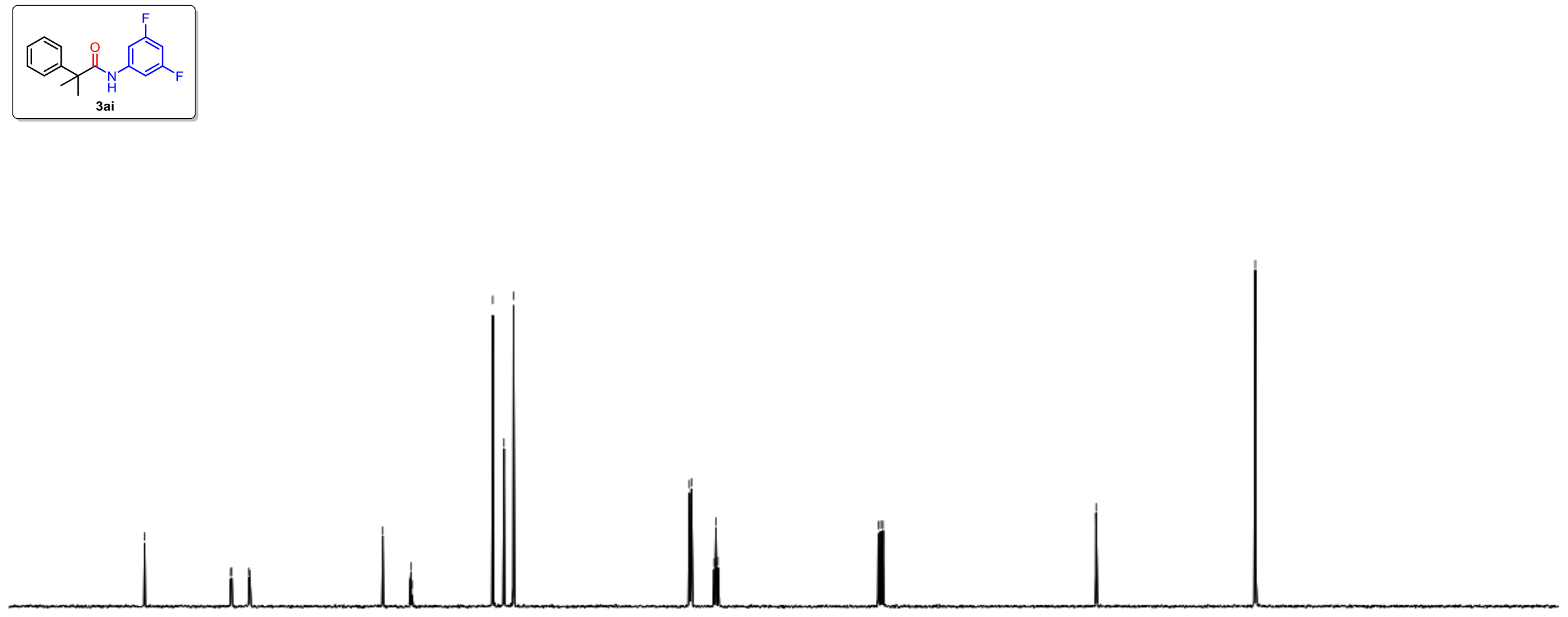

$190 \quad 180-170$

$150 \quad 140$

$130 \quad 120$

110

$100 \quad \begin{array}{ll}90 \\ \mathrm{f} 1(\mathrm{ppm})\end{array}$

$70 \quad 60$

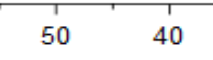


${ }^{19}$ F NMR Spectra $\left(375 \mathrm{MHz}, \mathrm{CDCl}_{3}\right.$ ) of compound 3ai
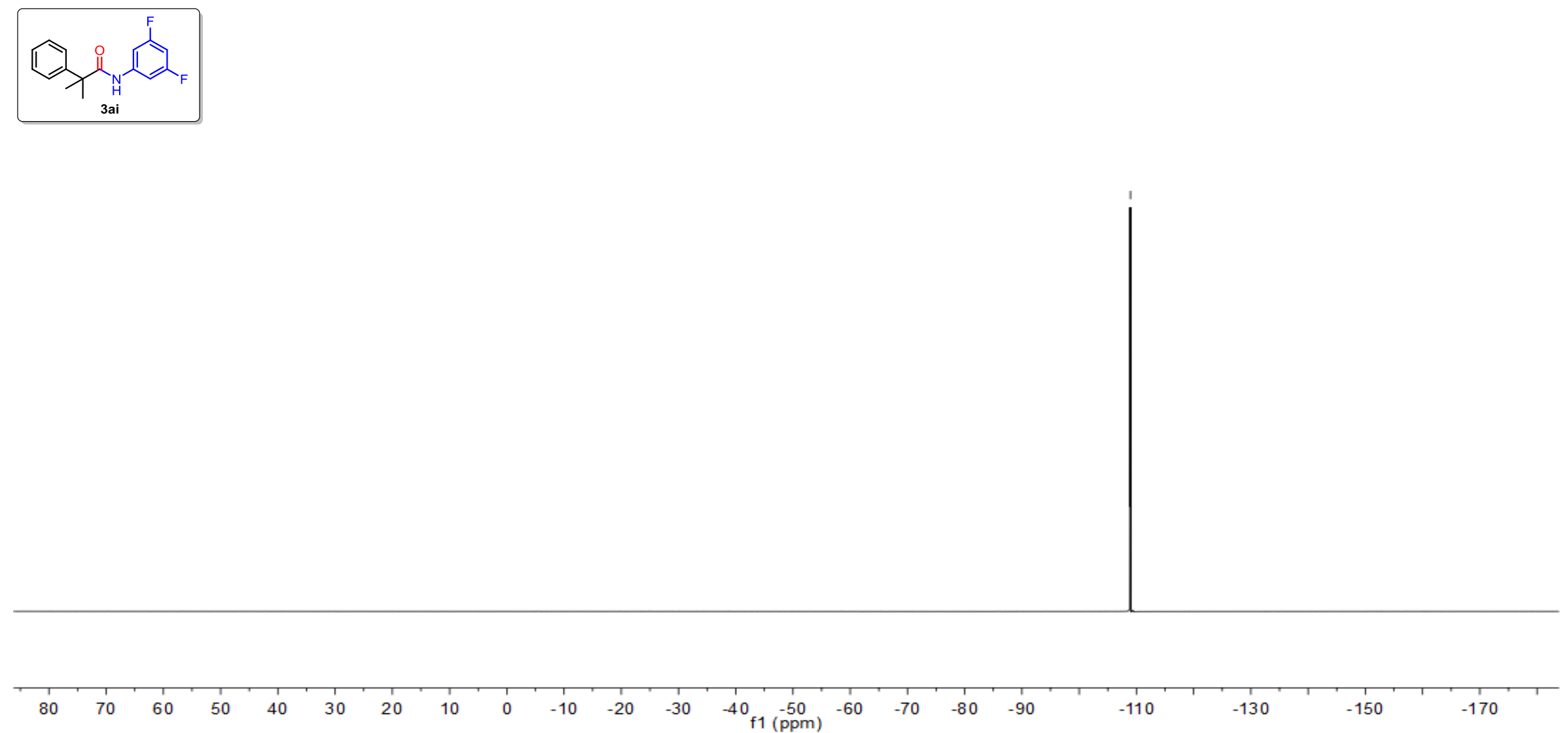
${ }^{1} \mathrm{H}$ NMR Spectra (400 MHz, $\mathrm{CDCl}_{3}$ ) of compound 3aj

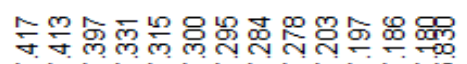

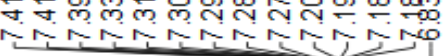
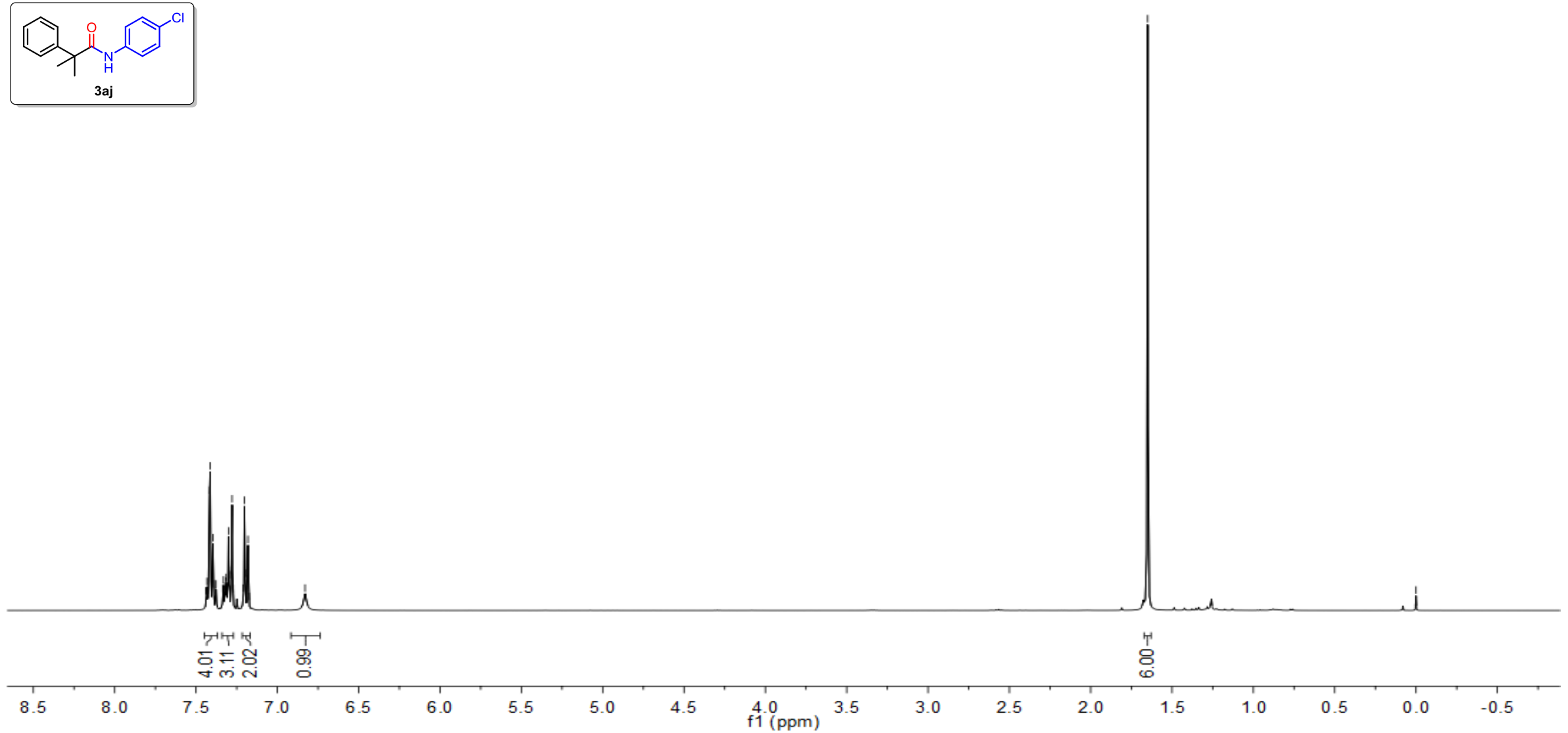
${ }^{13} \mathrm{C}$ NMR Spectra (101 MHz, $\mathrm{CDCl}_{3}$ ) of compound 3aj

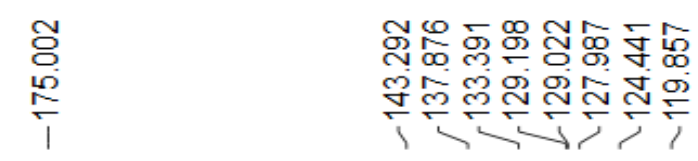

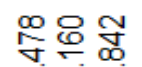

숭

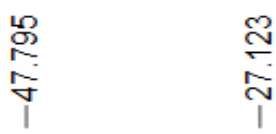
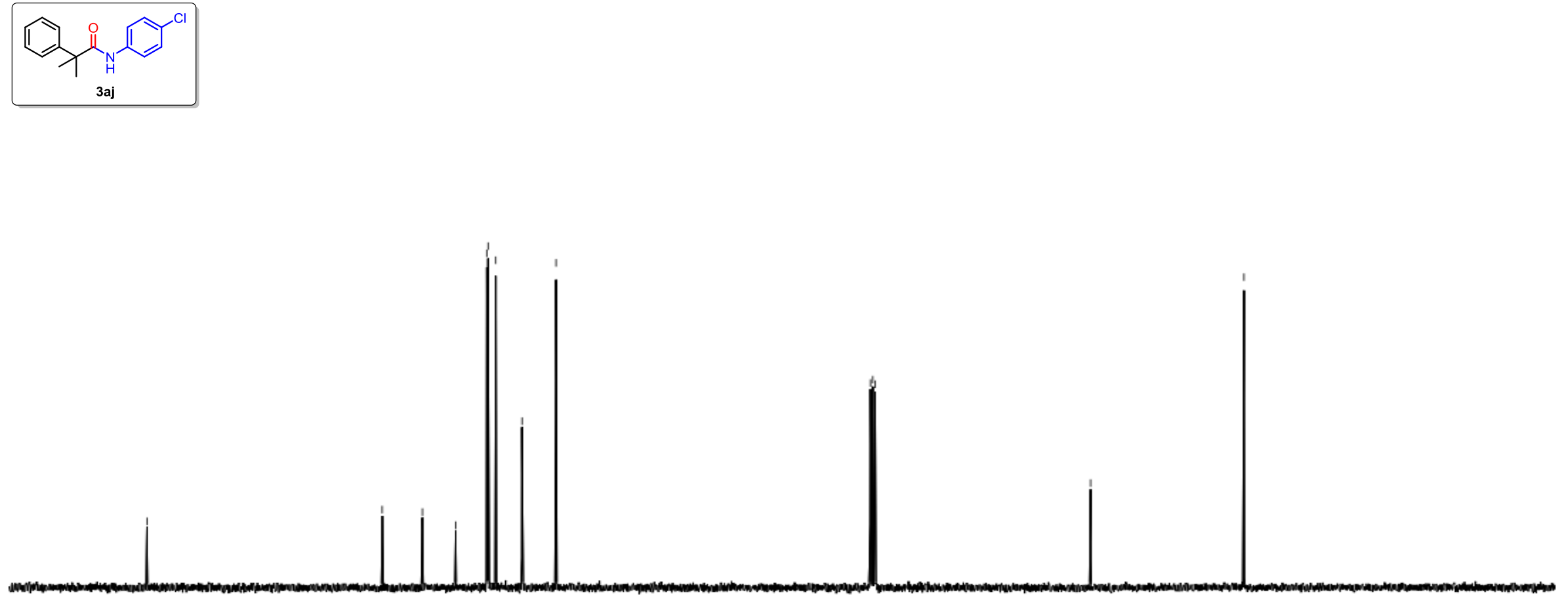

$190 \quad 180$ 
${ }^{1} \mathrm{H}$ NMR Spectra (400 MHz, $\mathrm{CDCl}_{3}$ ) of compound 3ak

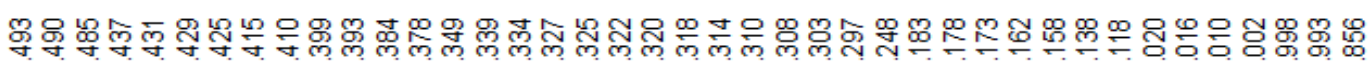

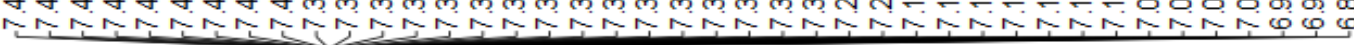
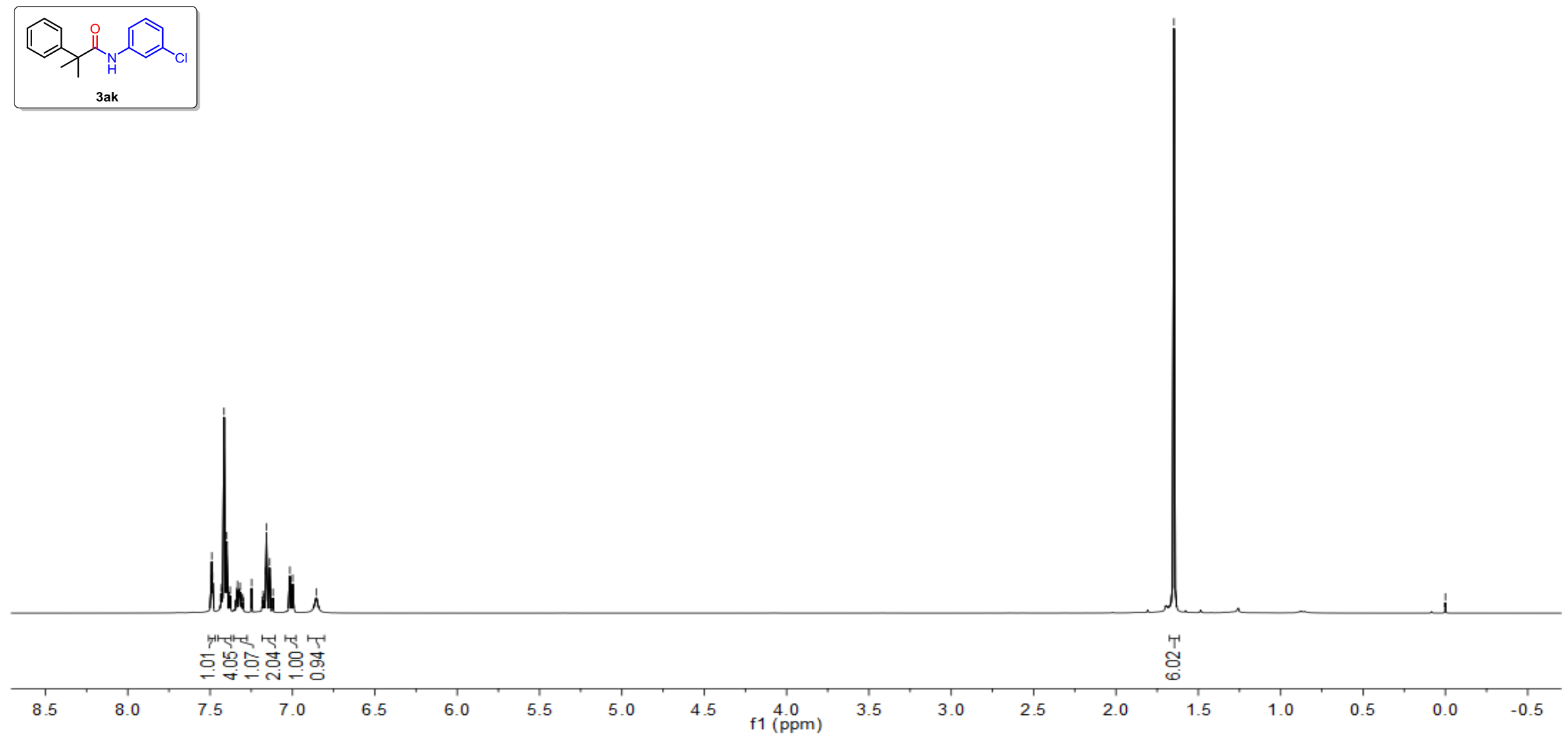
${ }^{13} \mathrm{C}$ NMR Spectra (101 MHz, $\mathrm{CDCl}_{3}$ ) of compound 3ak
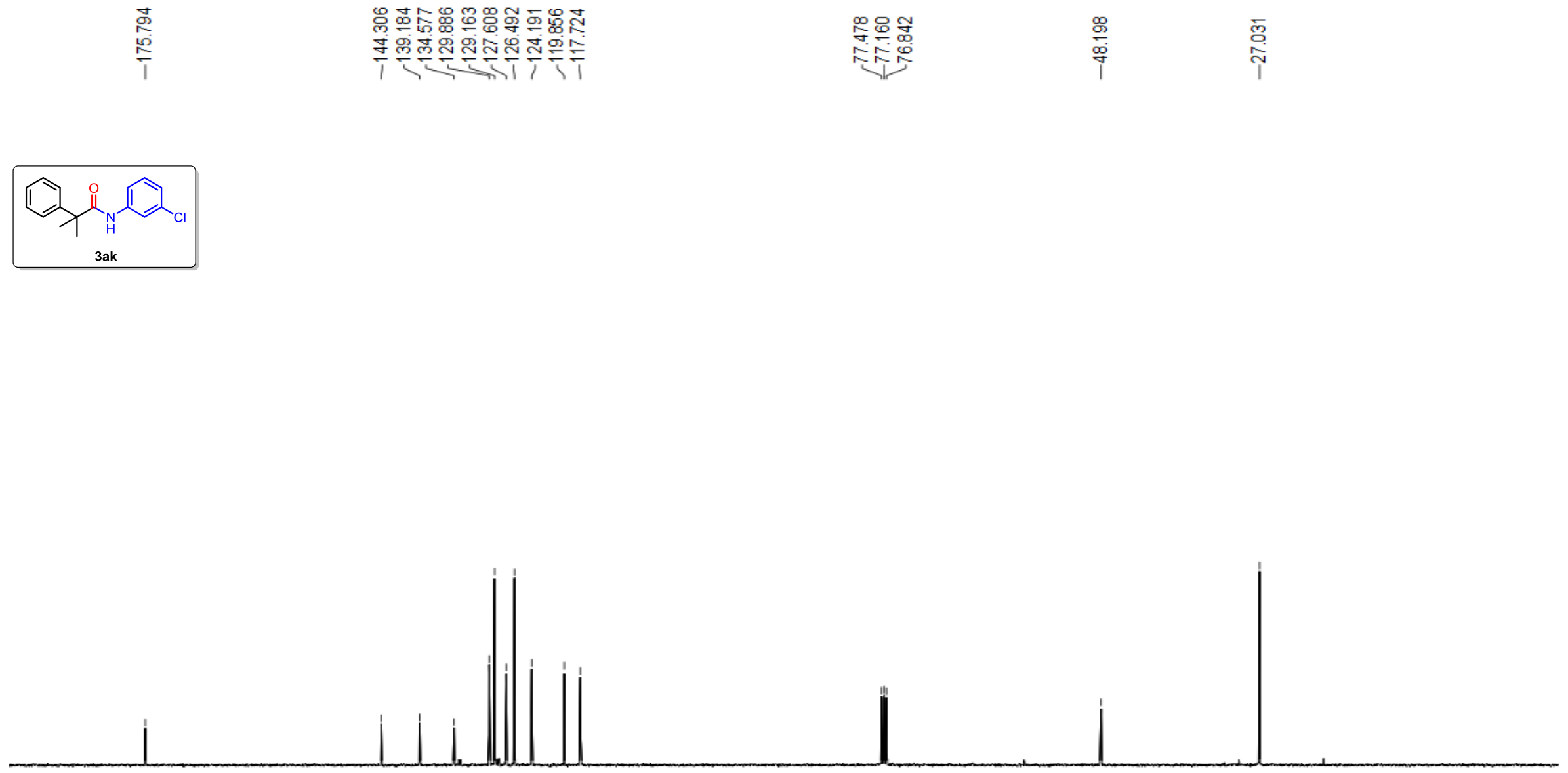

$190 \quad 180$

$130 \quad 120$

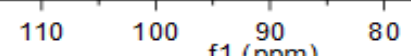

70

$60 \quad 50$

$40 \quad 30$

20

$10 \quad 0$ 
${ }^{1} \mathrm{H}$ NMR Spectra (400 MHz, $\mathrm{CDCl}_{3}$ ) of compound 3al

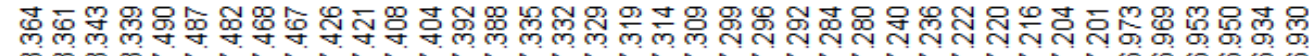

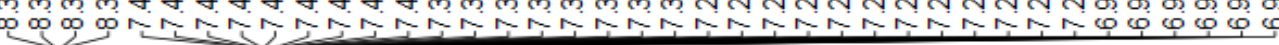
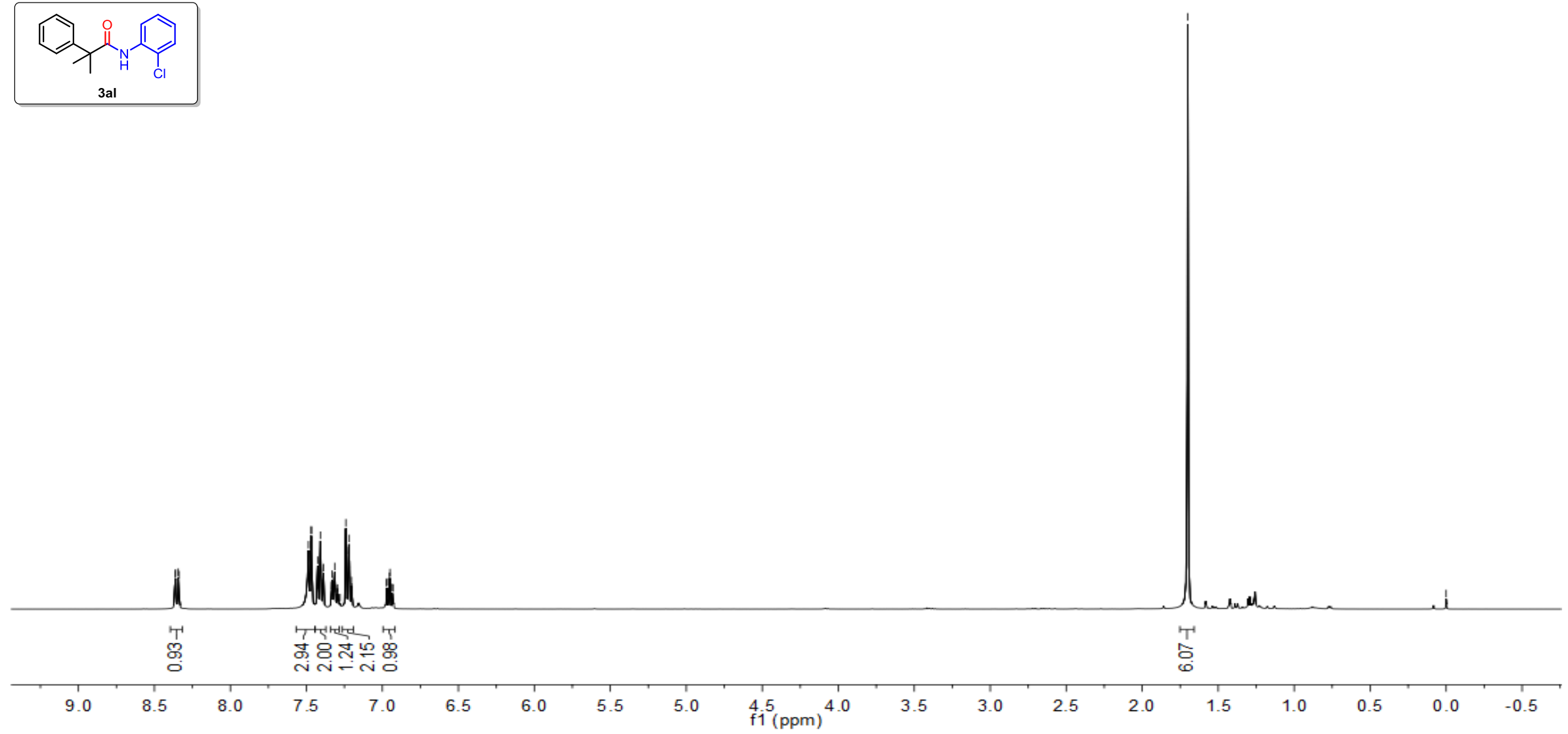
${ }^{13} \mathrm{C}$ NMR Spectra (101 MHz, $\mathrm{CDCl}_{3}$ ) of compound 3al
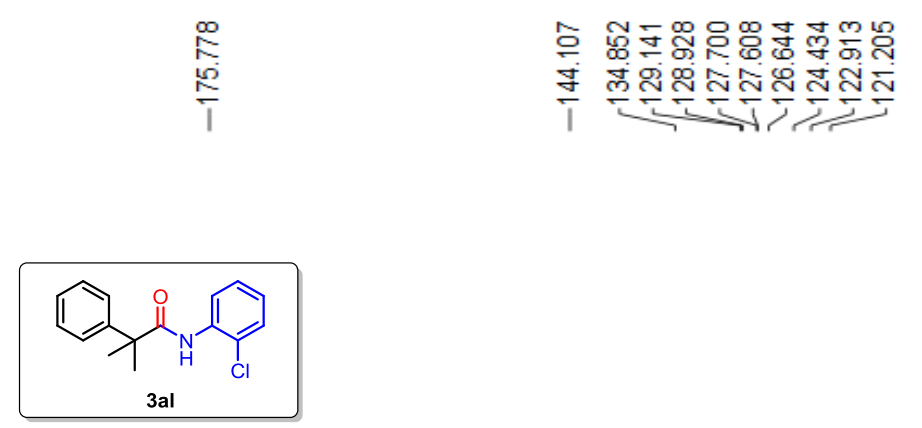

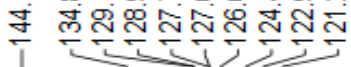

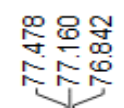

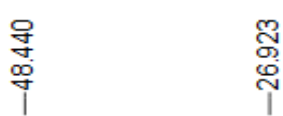

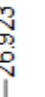

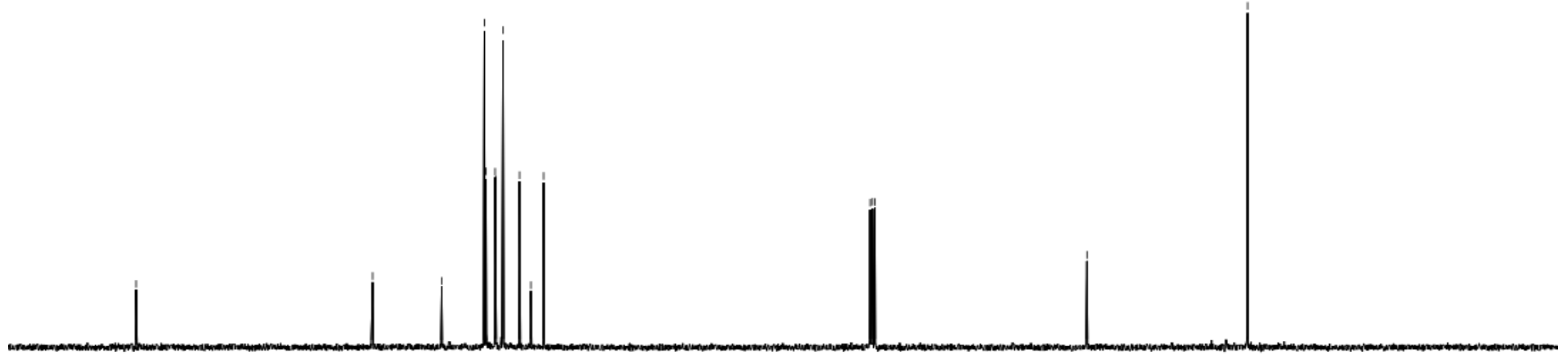

190

$160 \quad 150$

140

130

120

110

100

90080

70

60

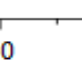

40

30

20 
${ }^{1} \mathrm{H}$ NMR Spectra $\left(400 \mathrm{MHz}, \mathrm{CDCl}_{3}\right)$ of compound 3am

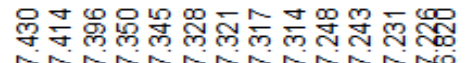

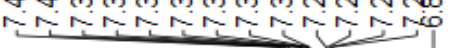
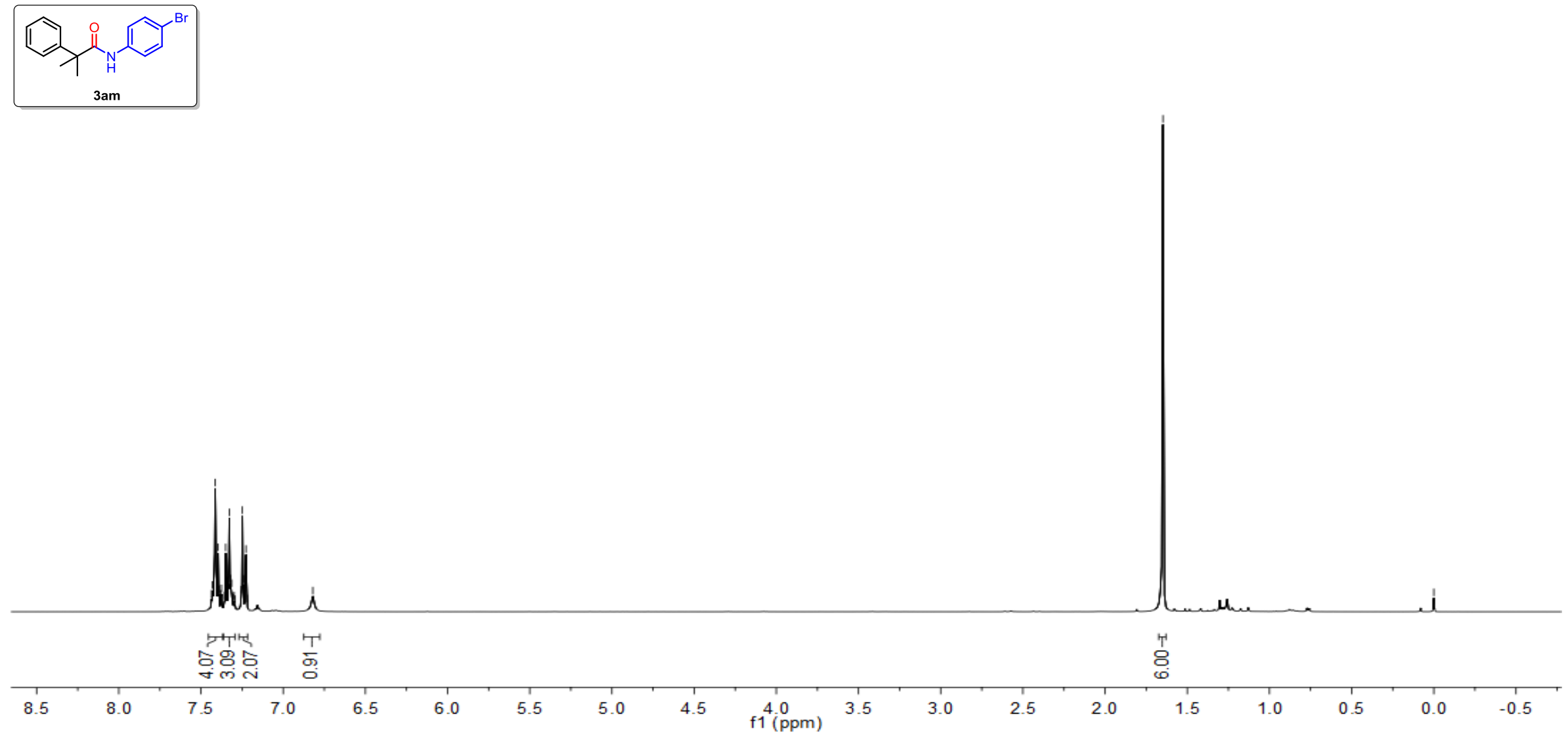
${ }^{13} \mathrm{C}$ NMR Spectra (101 MHz, $\mathrm{CDCl}_{3}$ ) of compound 3am
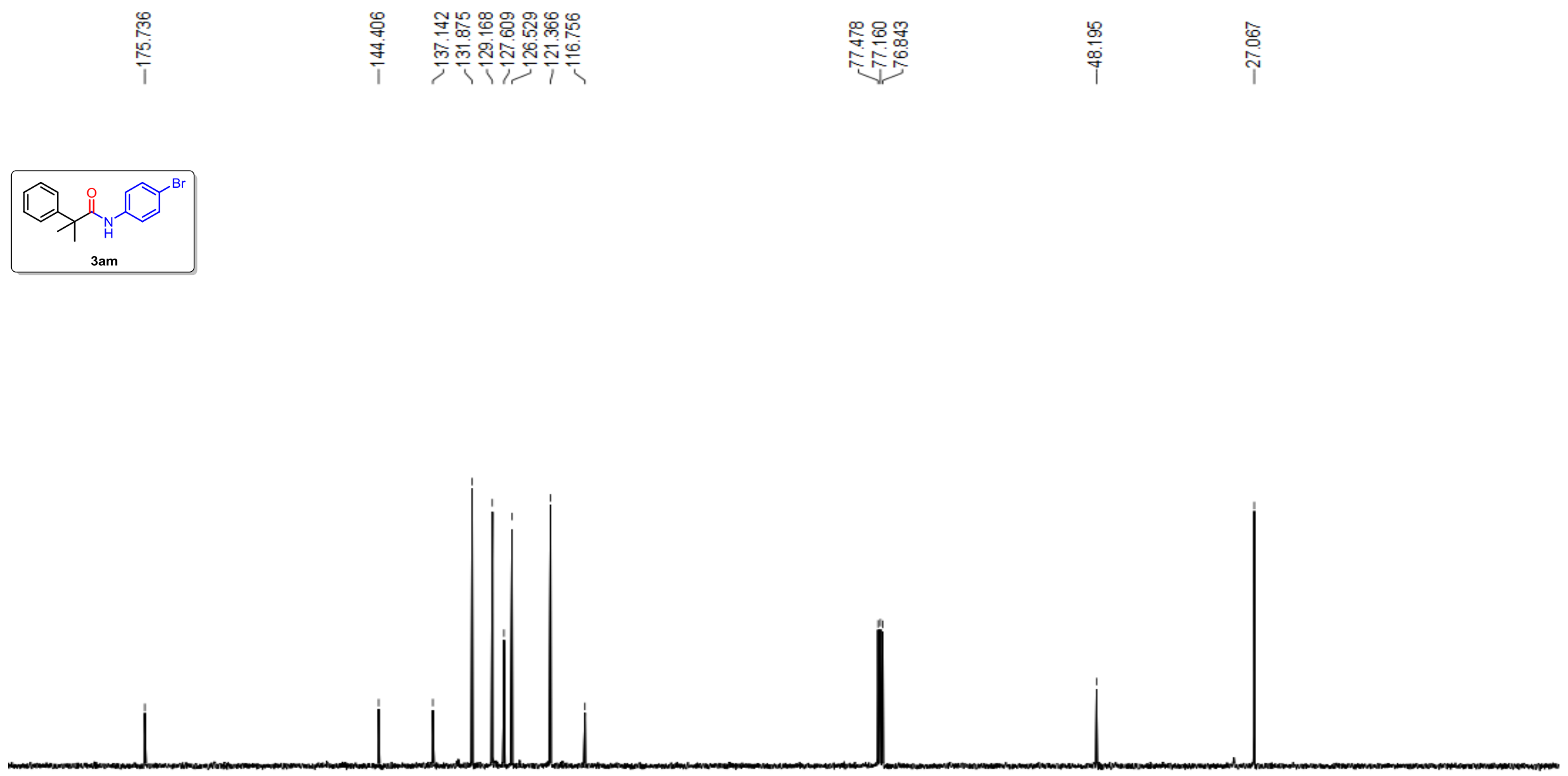

190180

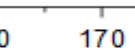

160

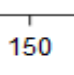

$140 \quad 130$

120

110

$100 \quad 90$

80

70

60 
${ }^{1} \mathrm{H}$ NMR Spectra (400 MHz, $\mathrm{CDCl}_{3}$ ) of compound 3an

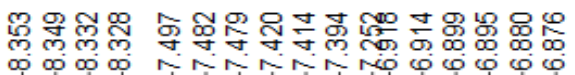
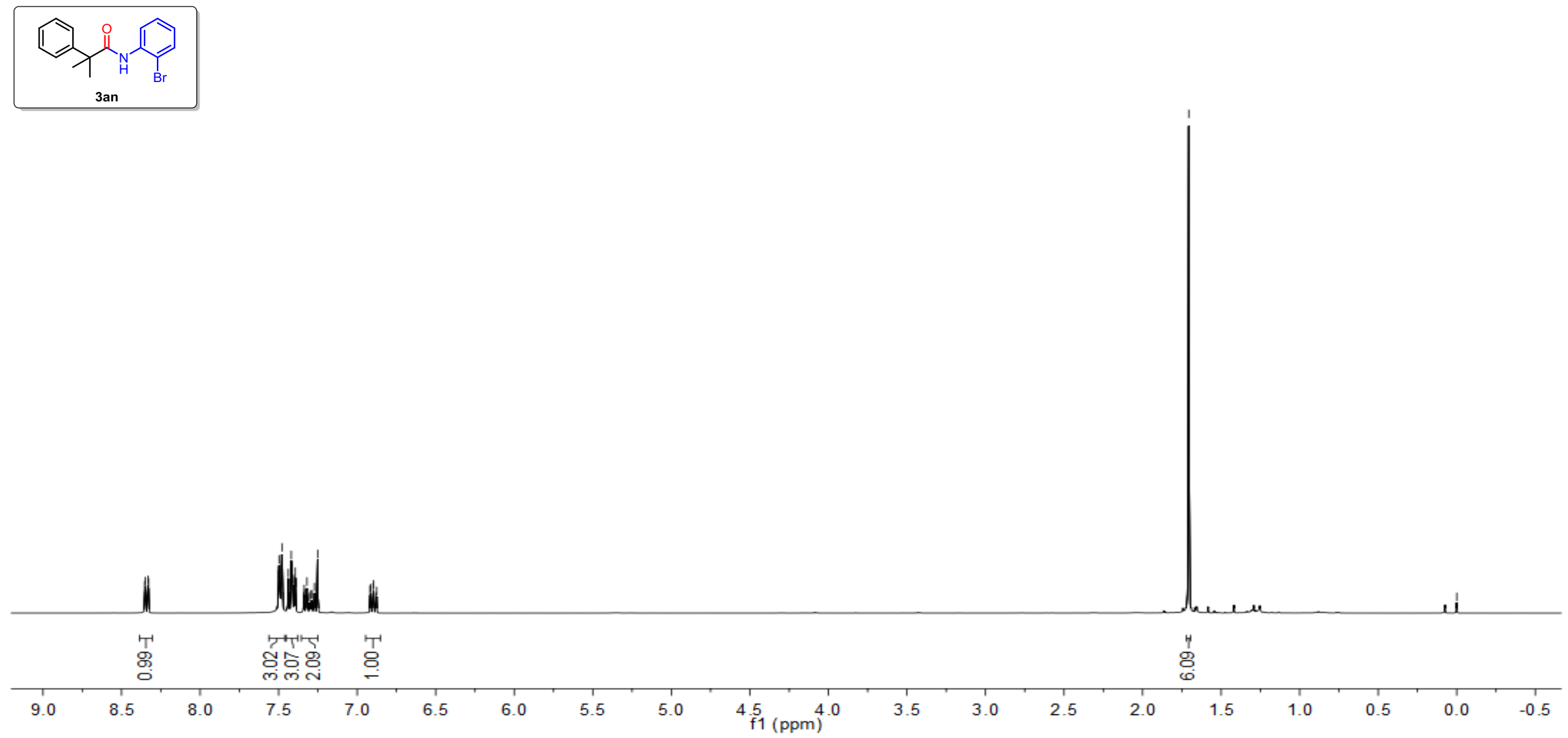
${ }^{13} \mathrm{C}$ NMR Spectra (101 MHz, $\mathrm{CDCl}_{3}$ ) of compound 3an
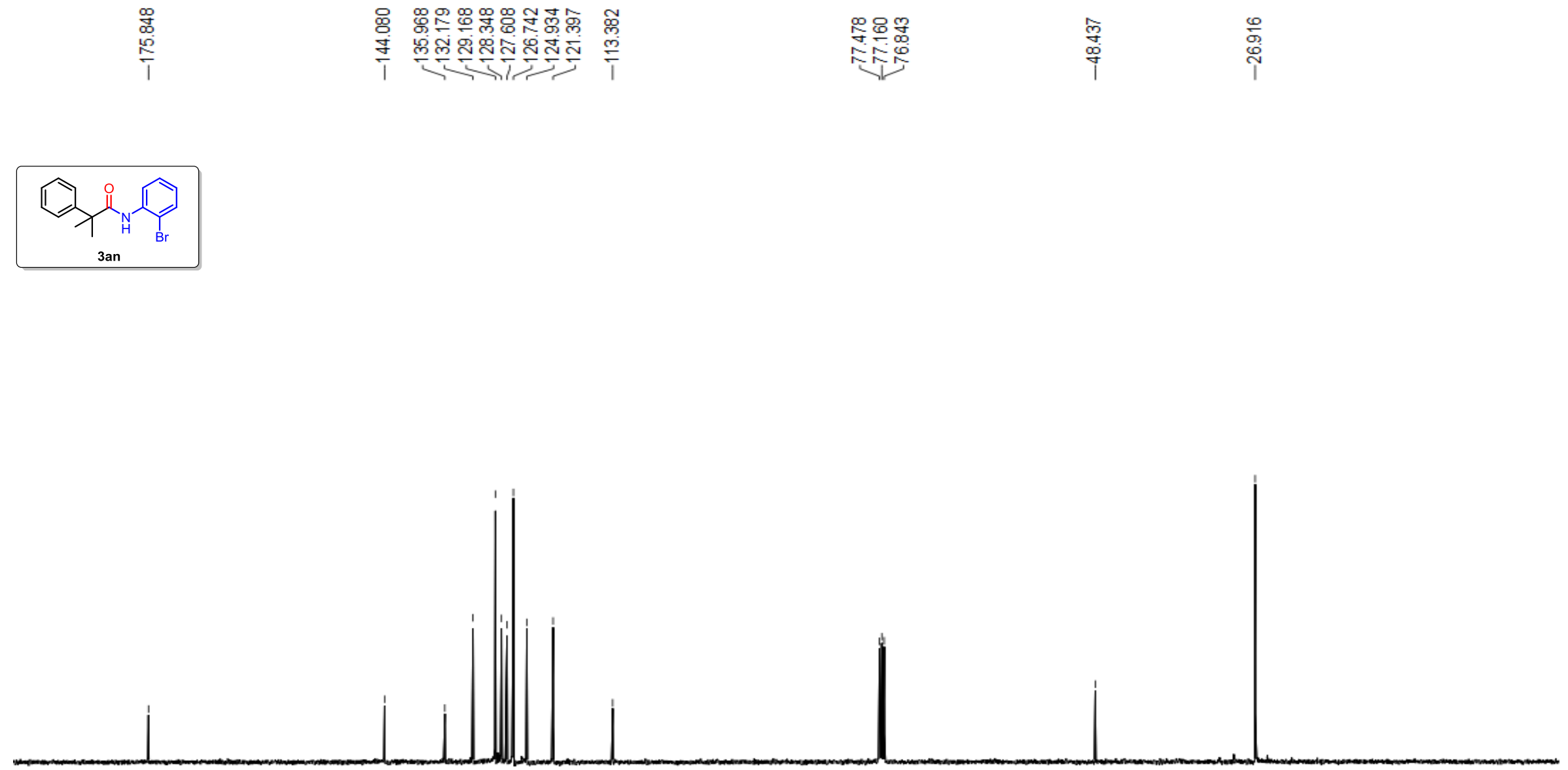
${ }^{1} \mathrm{H}$ NMR Spectra (400 MHz, $\mathrm{CDCl}_{3}$ ) of compound 3ao

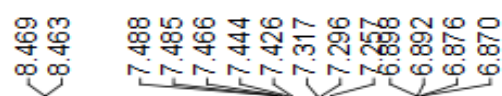

$\stackrel{1}{\frac{1}{2}} \quad$ 웅

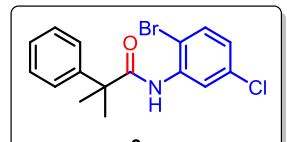

3ao

(1)

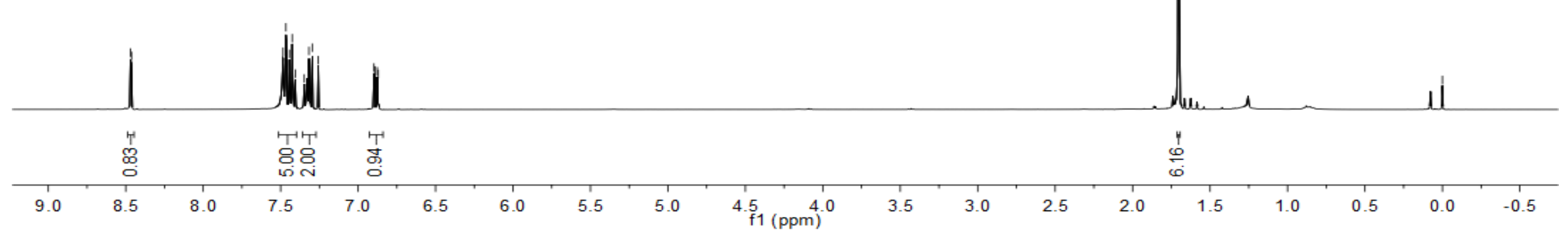


${ }^{13} \mathrm{C}$ NMR Spectra (101 MHz, $\mathrm{CDCl}_{3}$ ) of compound 3ao
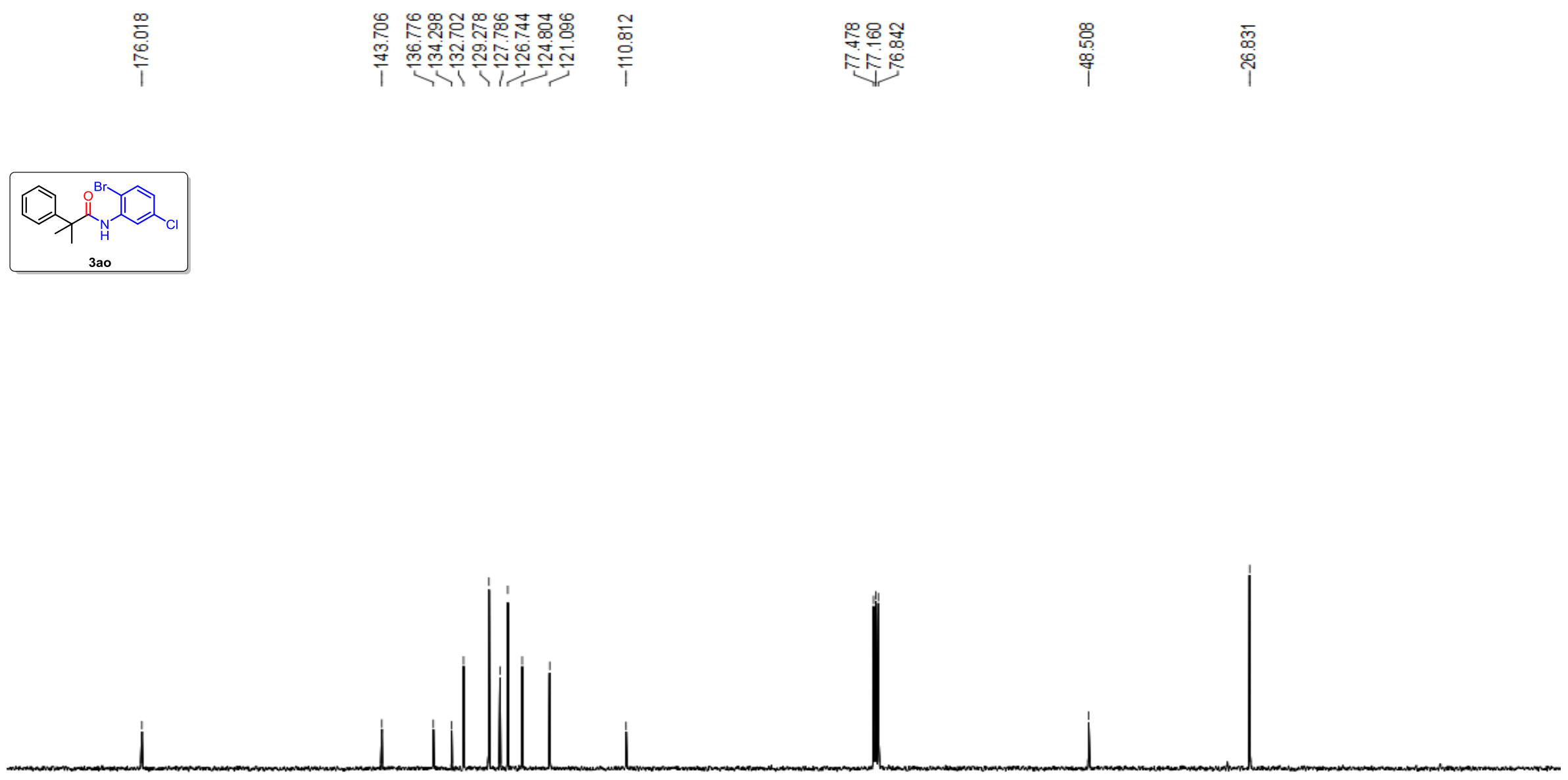

$190 \quad 180$

170 160

150

140

120

110

100

90,80

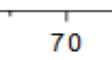

60

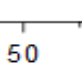

$40 \quad 30$

20 
${ }^{1} \mathrm{H}$ NMR Spectra (400 MHz, $\mathrm{CDCl}_{3}$ ) of compound 3ap

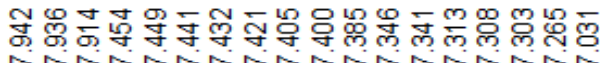

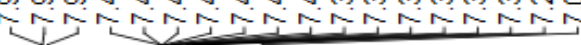
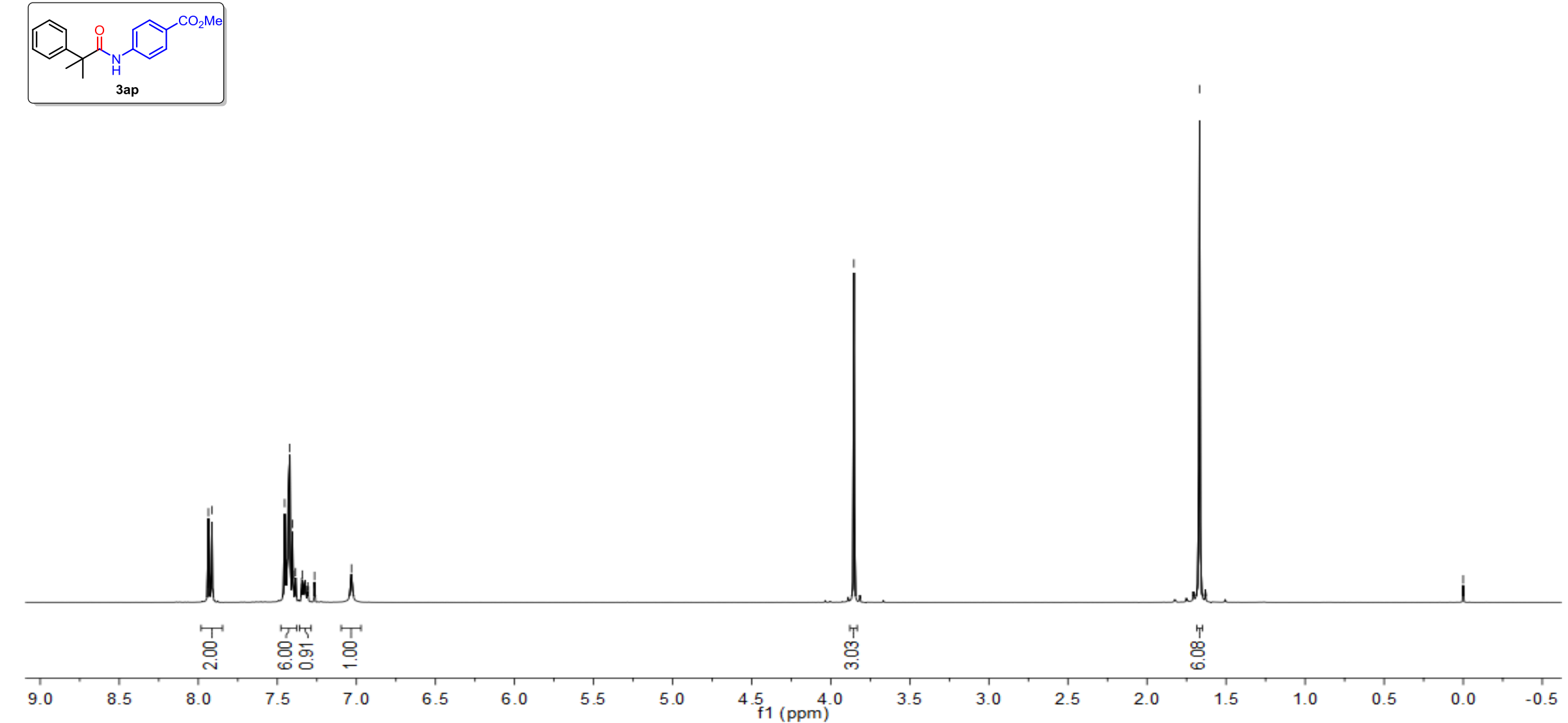
${ }^{13} \mathrm{C}$ NMR Spectra (101 MHz, $\mathrm{CDCl}_{3}$ ) of compound 3ap

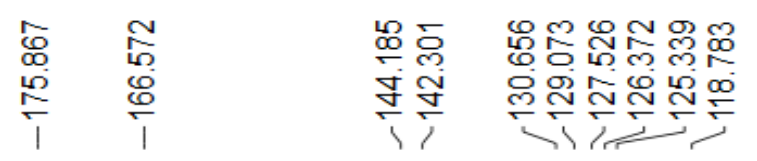

果 음요

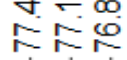

然尊

这

啘

1
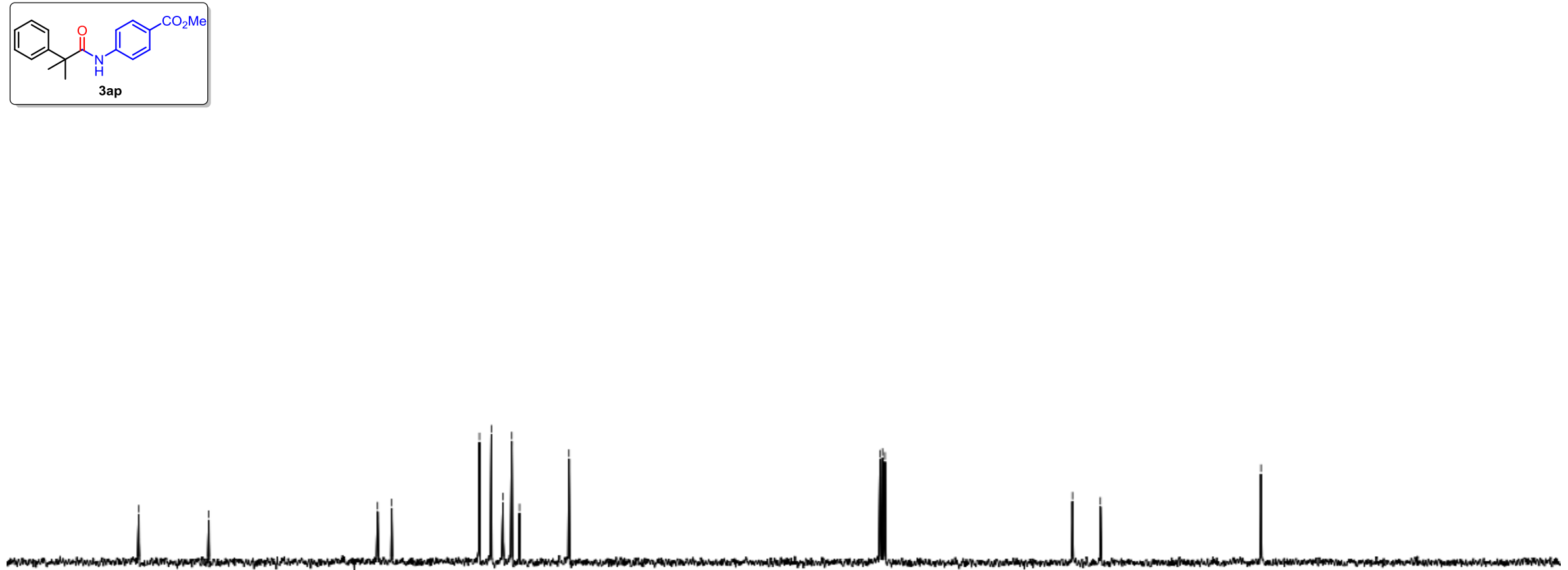
${ }^{1} \mathrm{H}$ NMR Spectra (400 MHz, $\mathrm{CDCl}_{3}$ ) of compound $\mathbf{3 a q}$

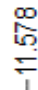

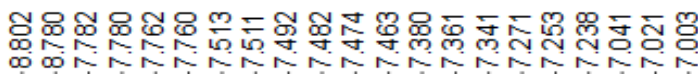

은 옹
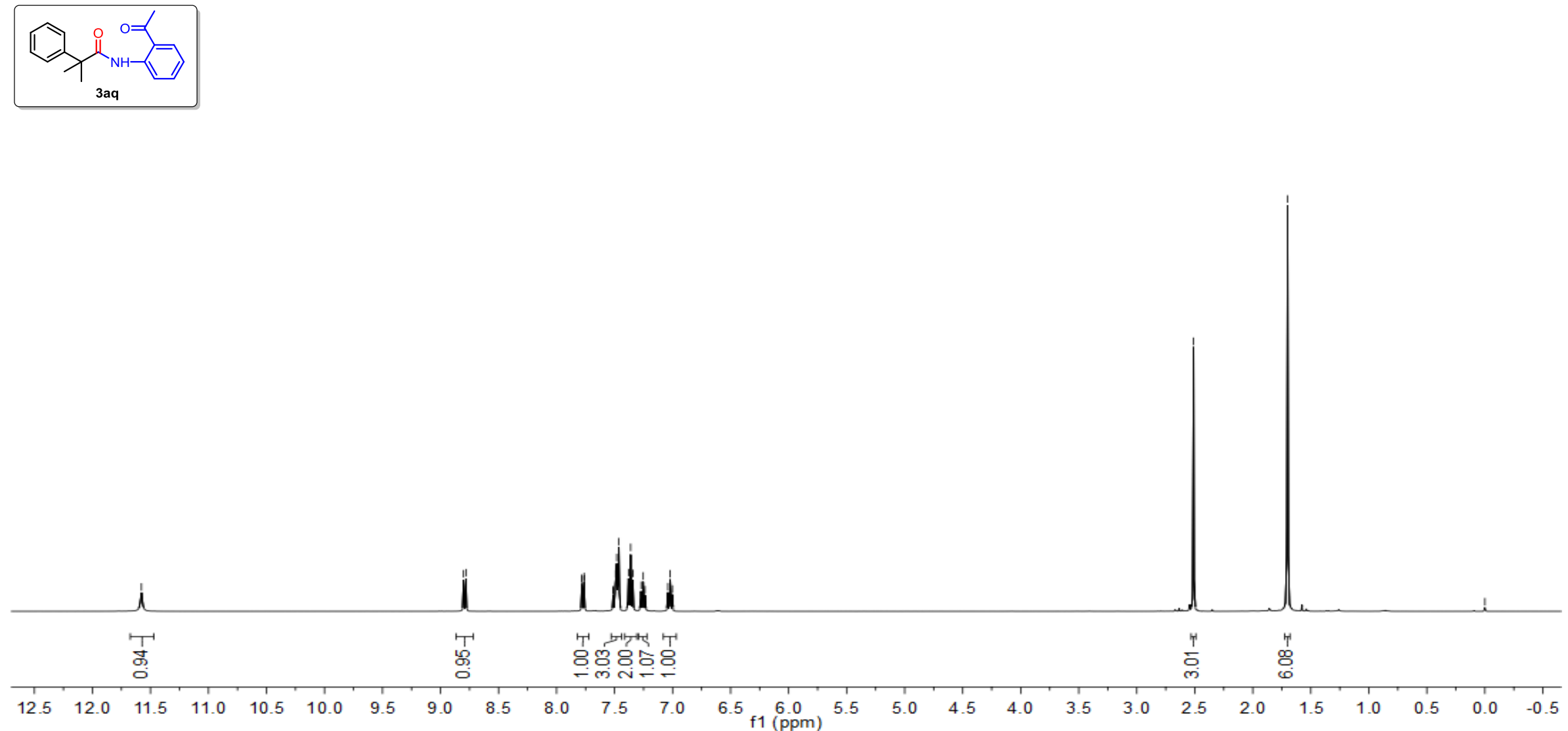
${ }^{13} \mathrm{C}$ NMR Spectra (101 MHz, $\mathrm{CDCl}_{3}$ ) of compound 3aq

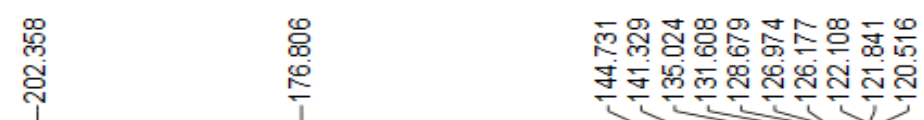

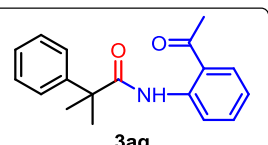

$3 a q$

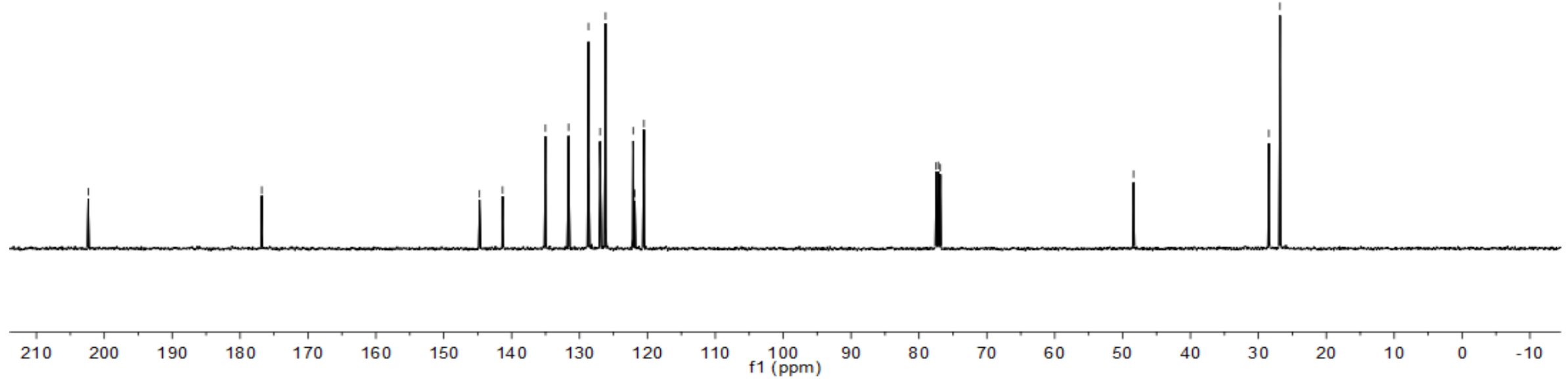


${ }^{1} \mathrm{H}$ NMR Spectra (400 MHz, $\mathrm{CDCl}_{3}$ ) of compound 3ar

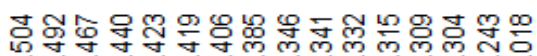

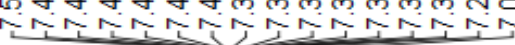
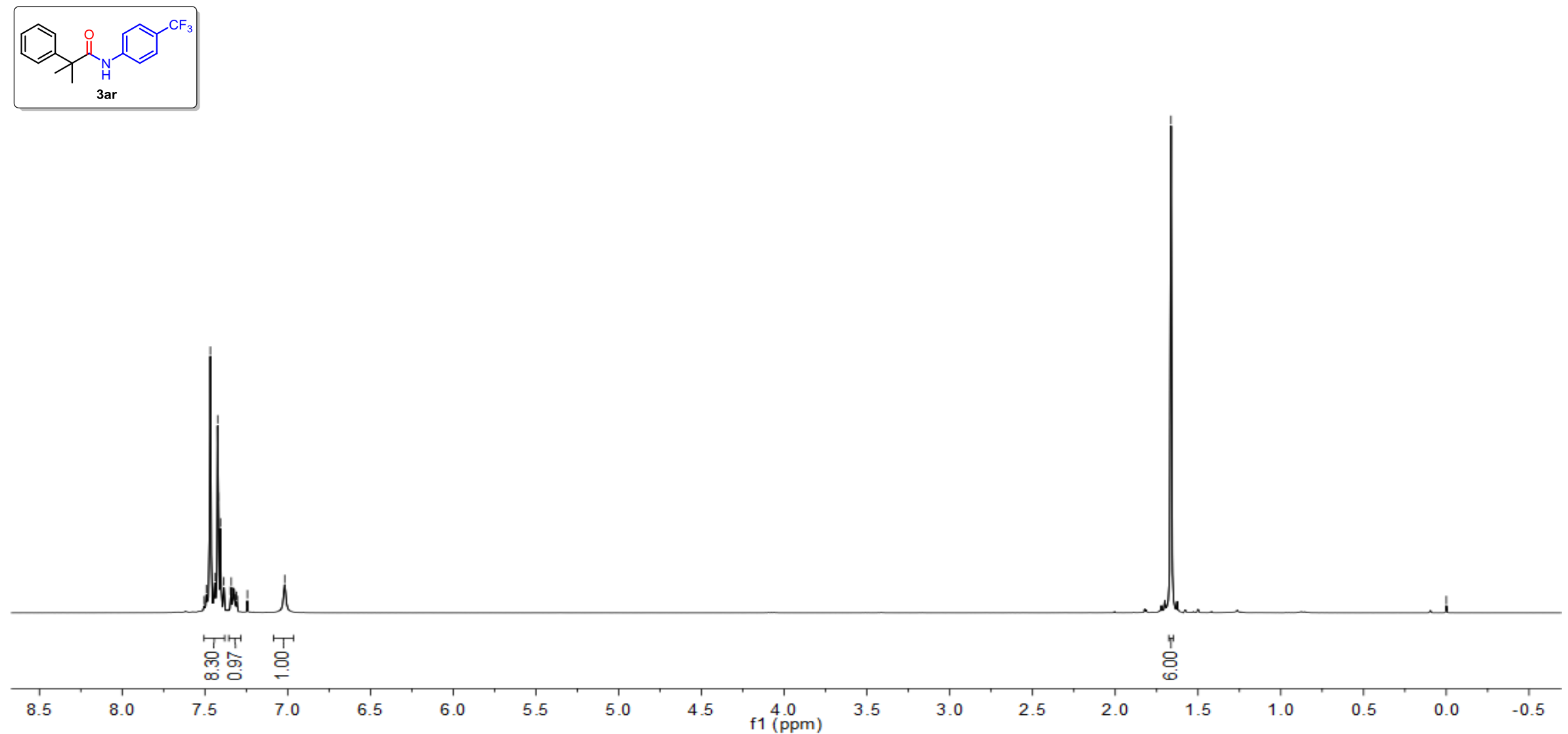
${ }^{13} \mathrm{C}$ NMR Spectra (101 MHz, $\mathrm{CDCl}_{3}$ ) of compound 3ar

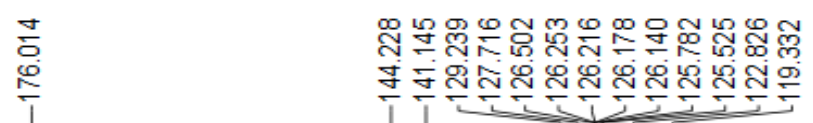

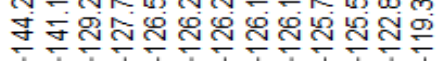

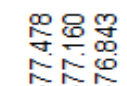

色

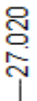
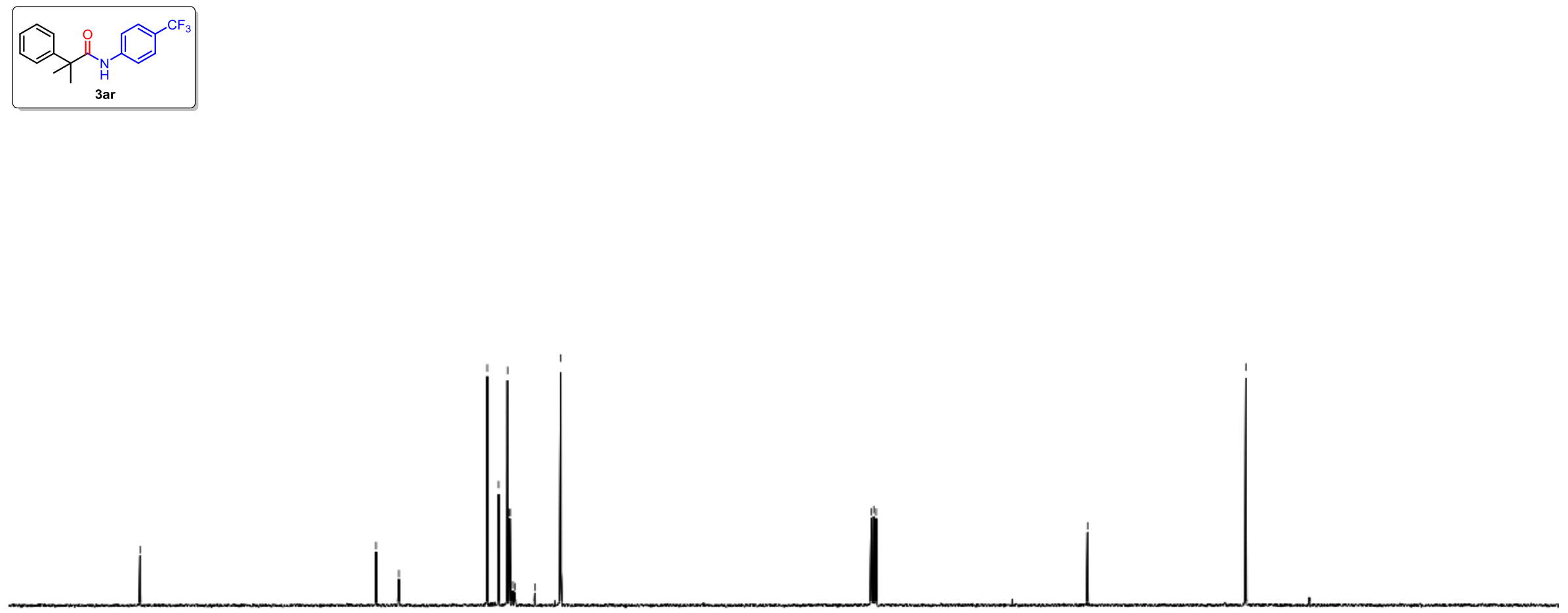

$190 \quad 180$

160

150

$140 \quad 130$

120

110

100

$\stackrel{1}{90} 80$

70

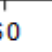

50 
${ }^{19} \mathrm{~F}$ NMR Spectra (375 MHz, $\mathrm{CDCl}_{3}$ ) of compound 3ar

$$
\underset{\substack{i \\ i}}{\stackrel{\infty}{7}}
$$
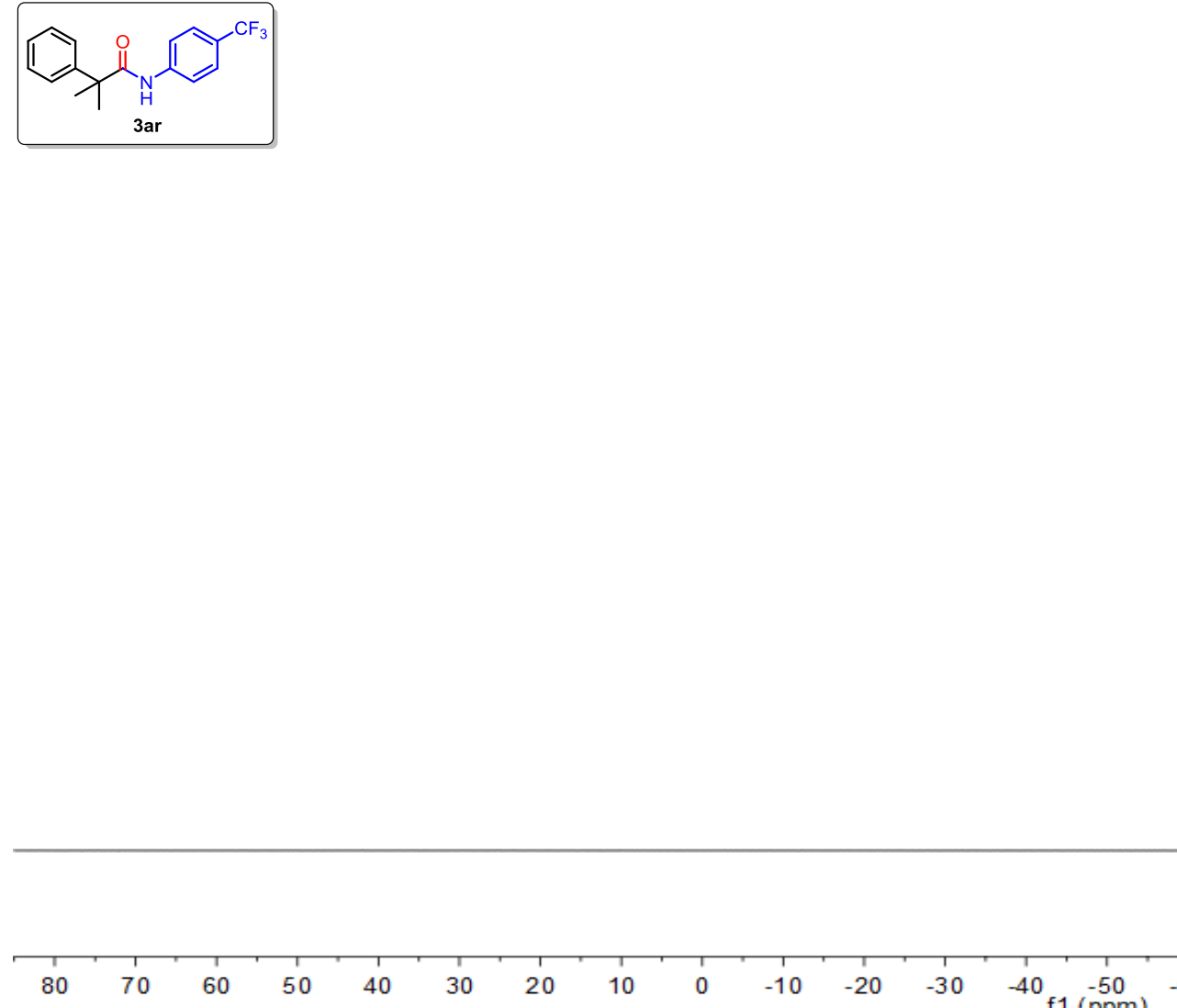

$-40 \begin{gathered}-50 \\ \mathrm{f} 1(\mathrm{ppm})\end{gathered}$

$\begin{array}{ccc}-70 & -80 & -90\end{array}$

$-110$

$-130$

$-150$ 
${ }^{1} \mathrm{H}$ NMR Spectra (400 MHz, $\mathrm{CDCl}_{3}$ ) of compound 3as

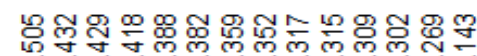

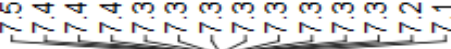
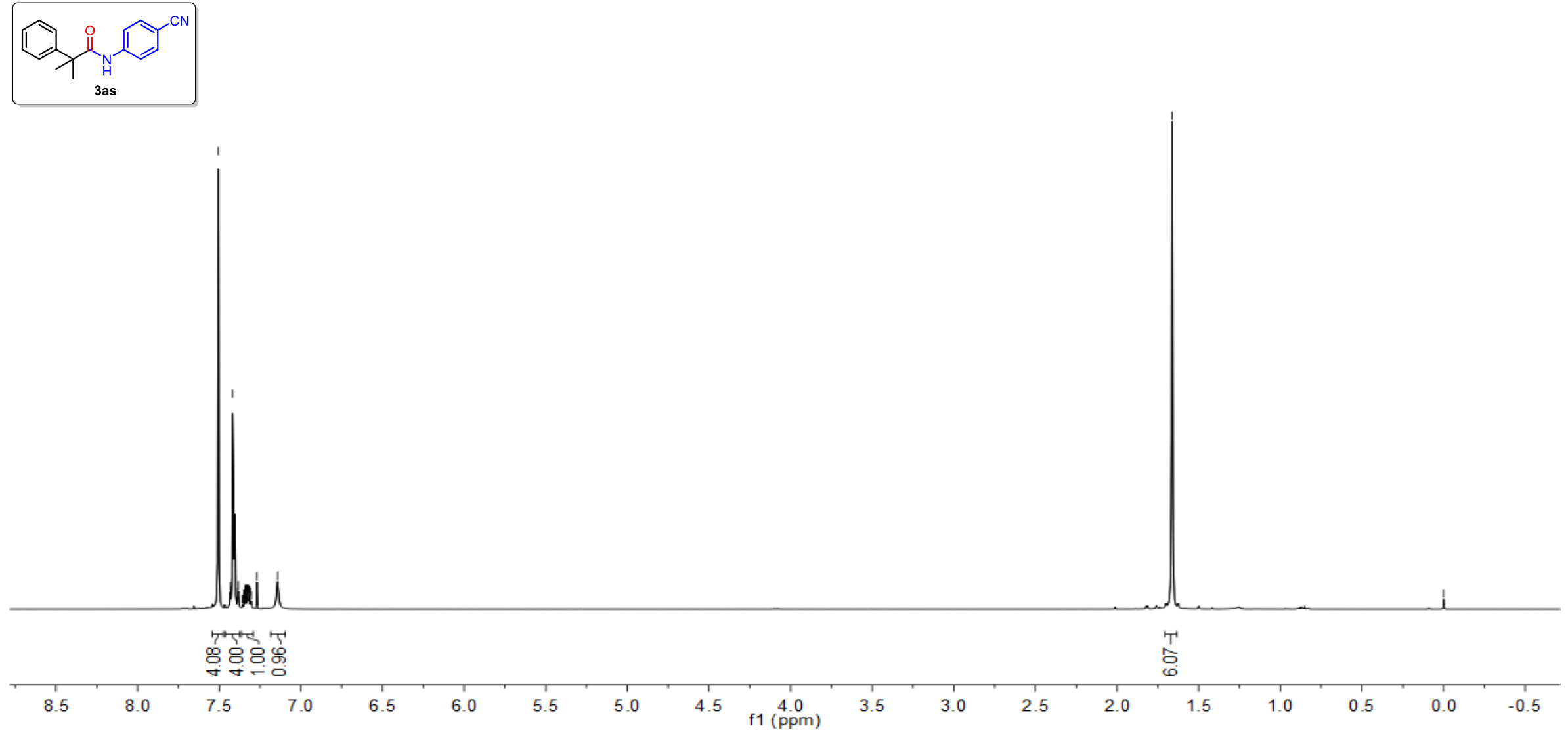
${ }^{13} \mathrm{C}$ NMR Spectra $\left(101 \mathrm{MHz}, \mathrm{CDCl}_{3}\right)$ of compound 3as

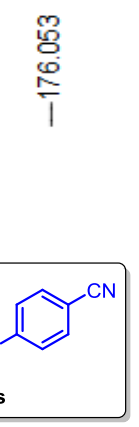

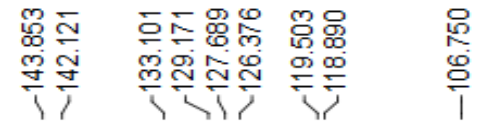
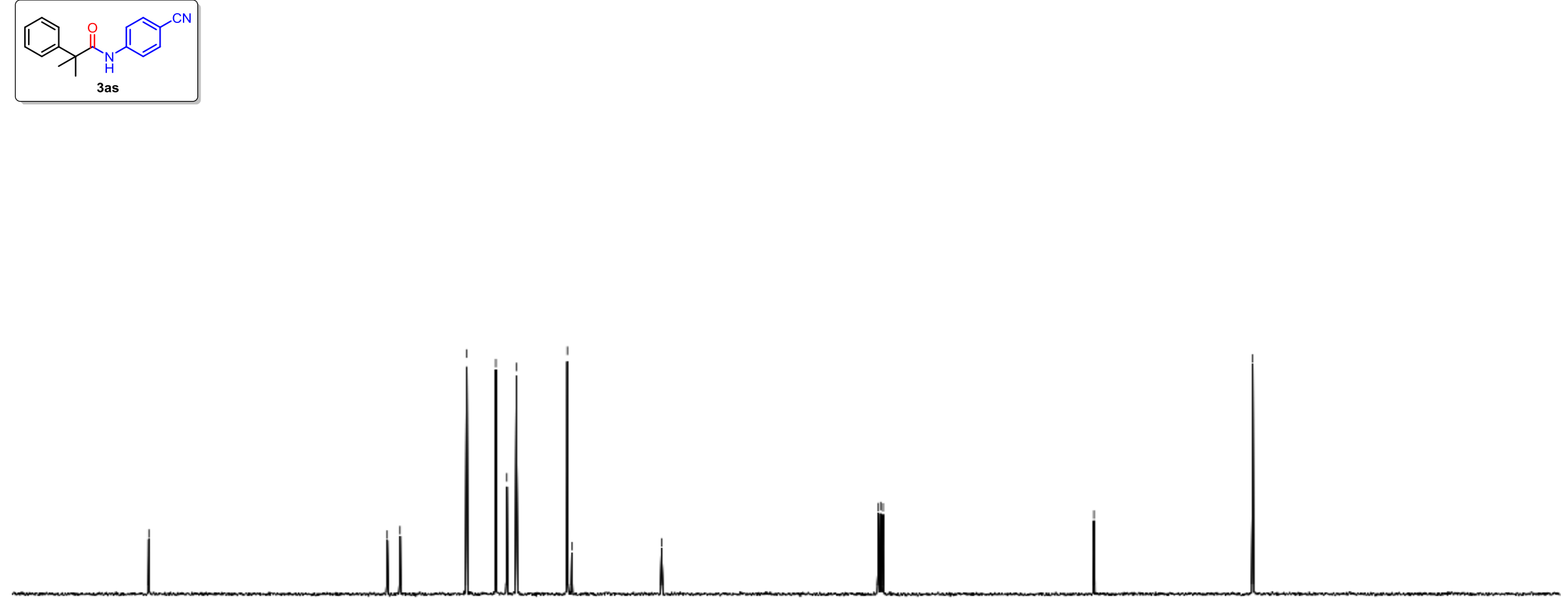

$190 \quad 180$

150

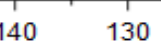

120

110

100990

$80 \quad 70$
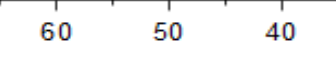

20 


\section{${ }^{1} \mathrm{H}$ NMR Spectra (400 MHz, $\mathrm{CDCl}_{3}$ ) of compound 3at}
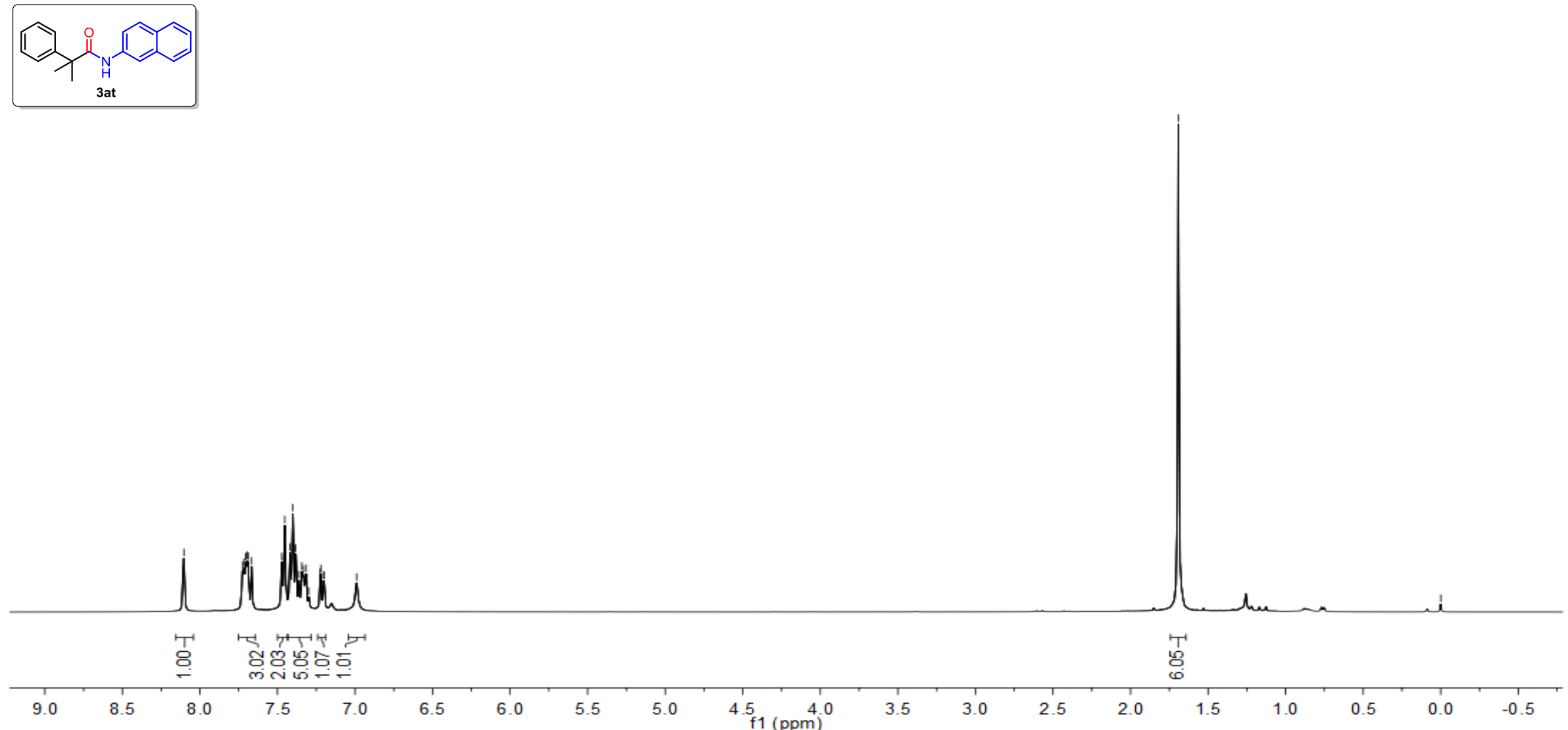
${ }^{13} \mathrm{C}$ NMR Spectra (101 MHz, $\mathrm{CDCl}_{3}$ ) of compound 3at

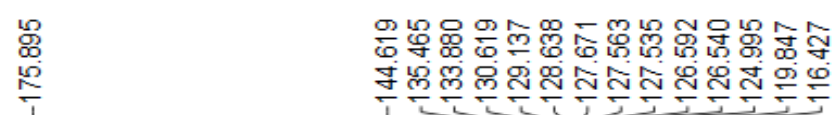

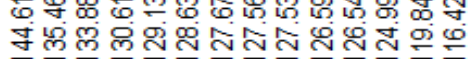

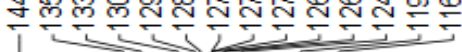
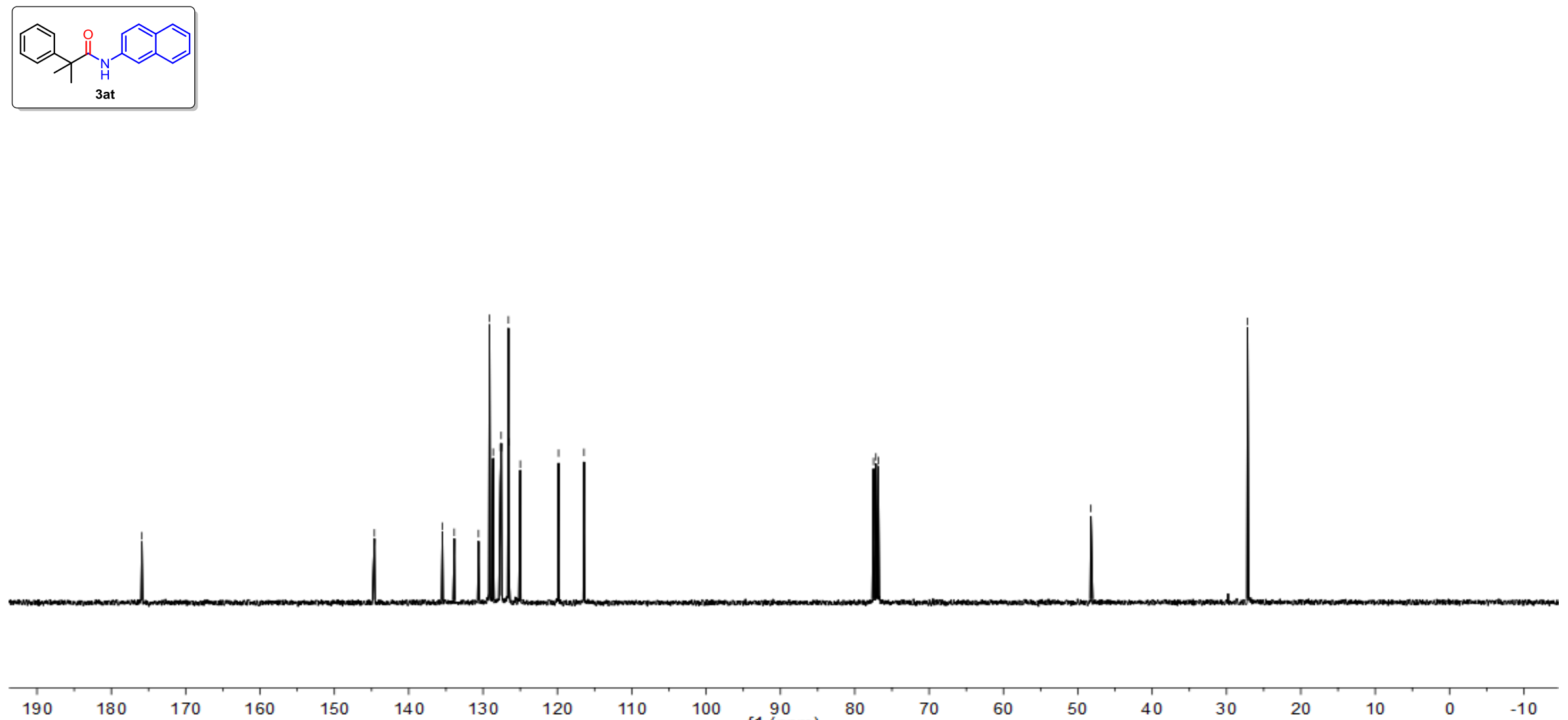

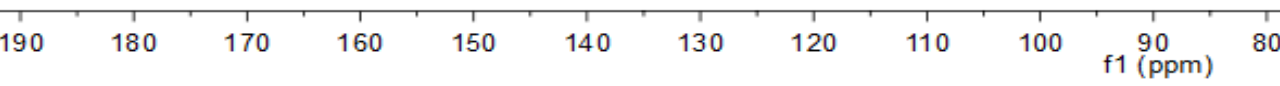

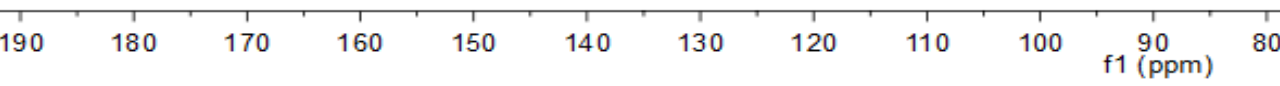

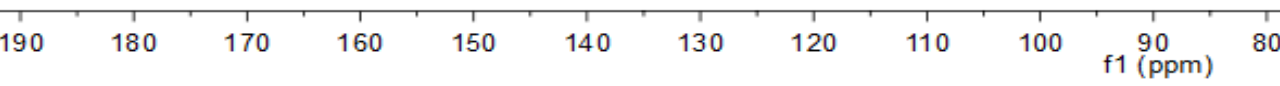

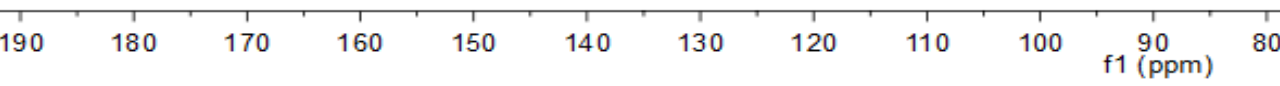


${ }^{1} \mathrm{H}$ NMR Spectra (400 MHz, $\mathrm{CDCl}_{3}$ ) of compound 3au

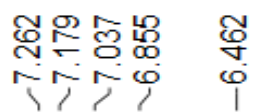

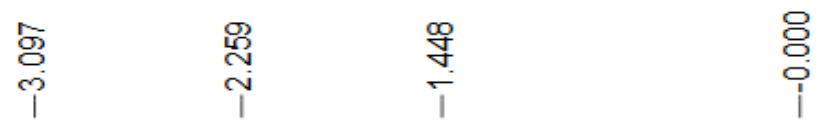
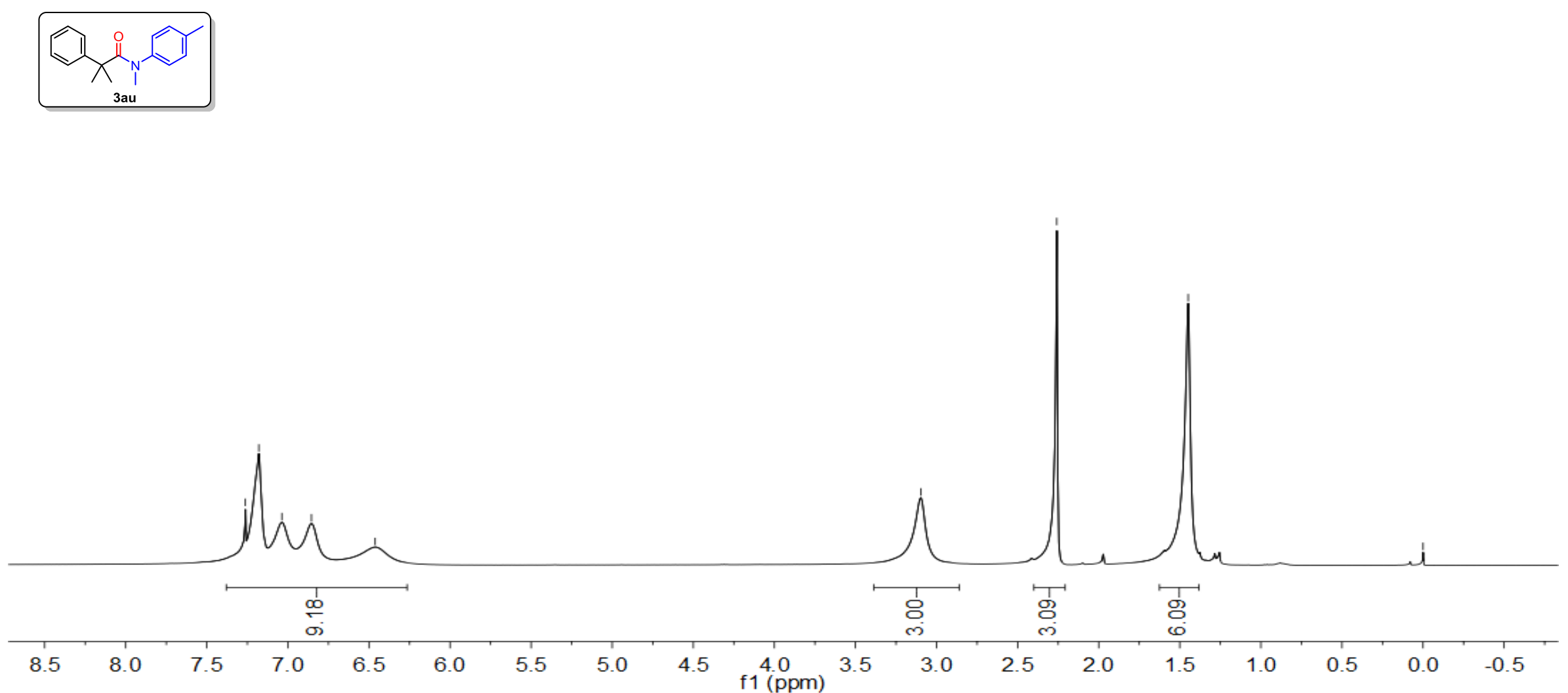
${ }^{13} \mathrm{C}$ NMR Spectra (101 MHz, $\mathrm{CDCl}_{3}$ ) of compound 3au

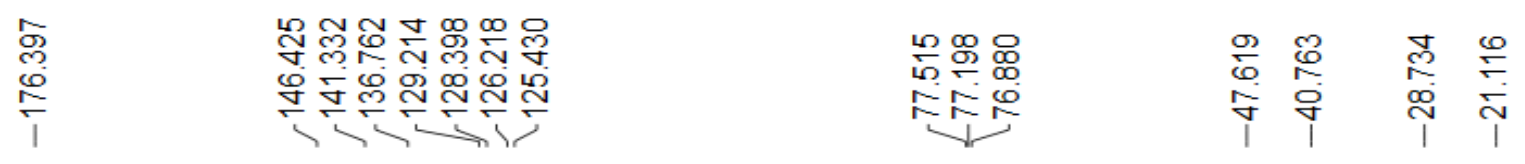
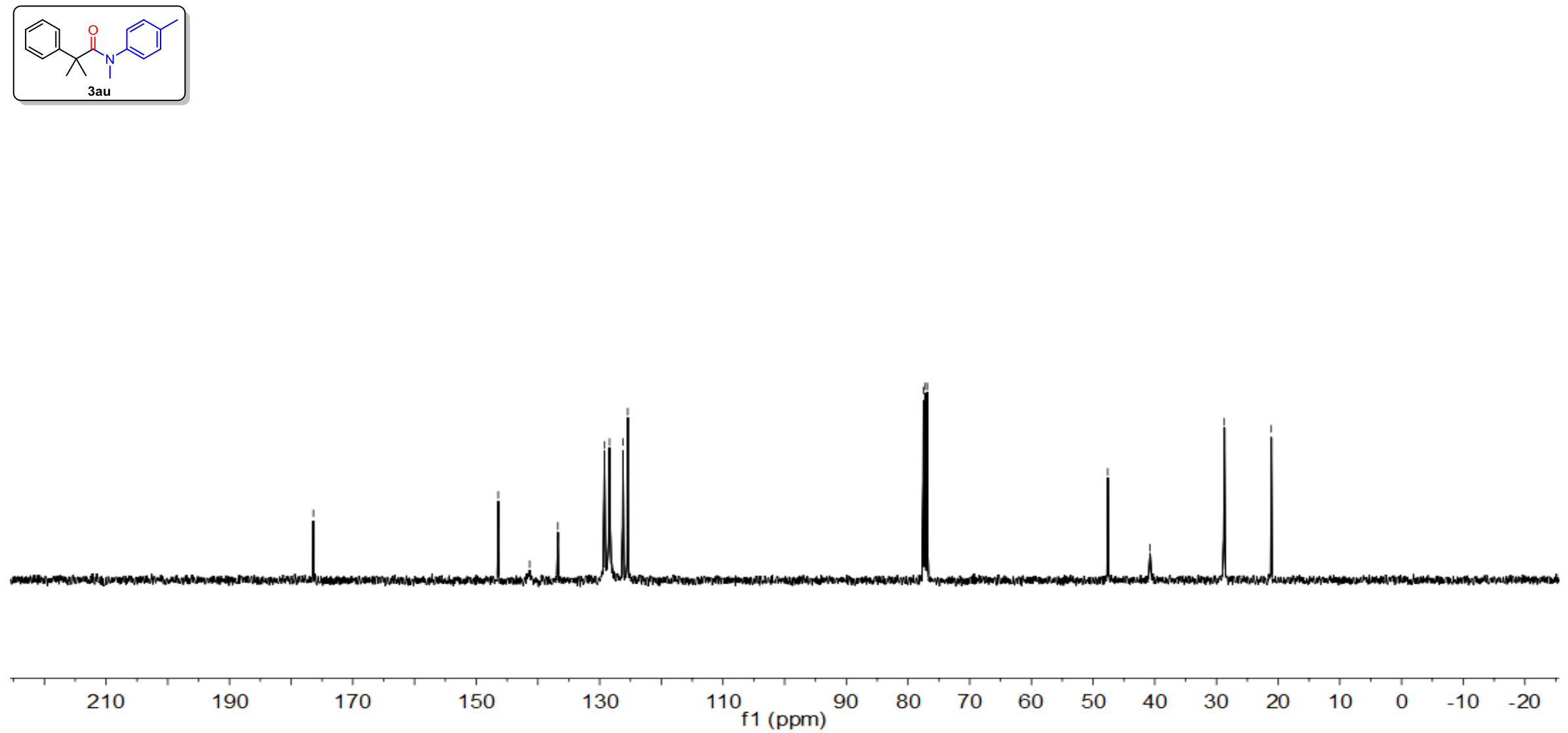
${ }^{1} \mathrm{H}$ NMR Spectra $\left(600 \mathrm{MHz}, \mathrm{CDCl}_{3}\right.$ ) of compound $\mathbf{3 a v}$

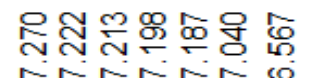

rininis
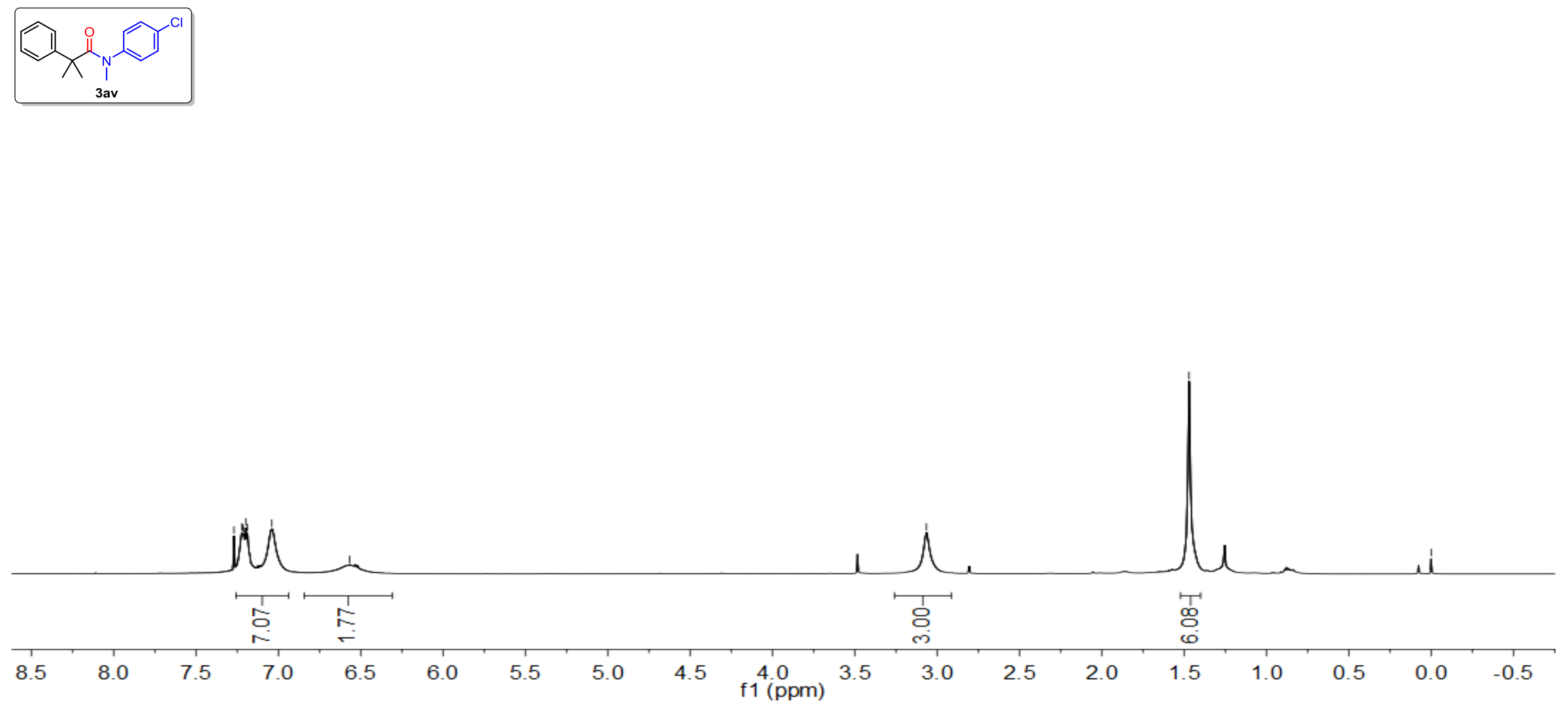
${ }^{13} \mathrm{C}$ NMR Spectra (151 MHz, $\mathrm{CDCl}_{3}$ ) of compound 3av

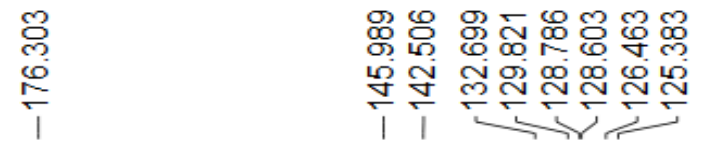

№ㅇํㅇ
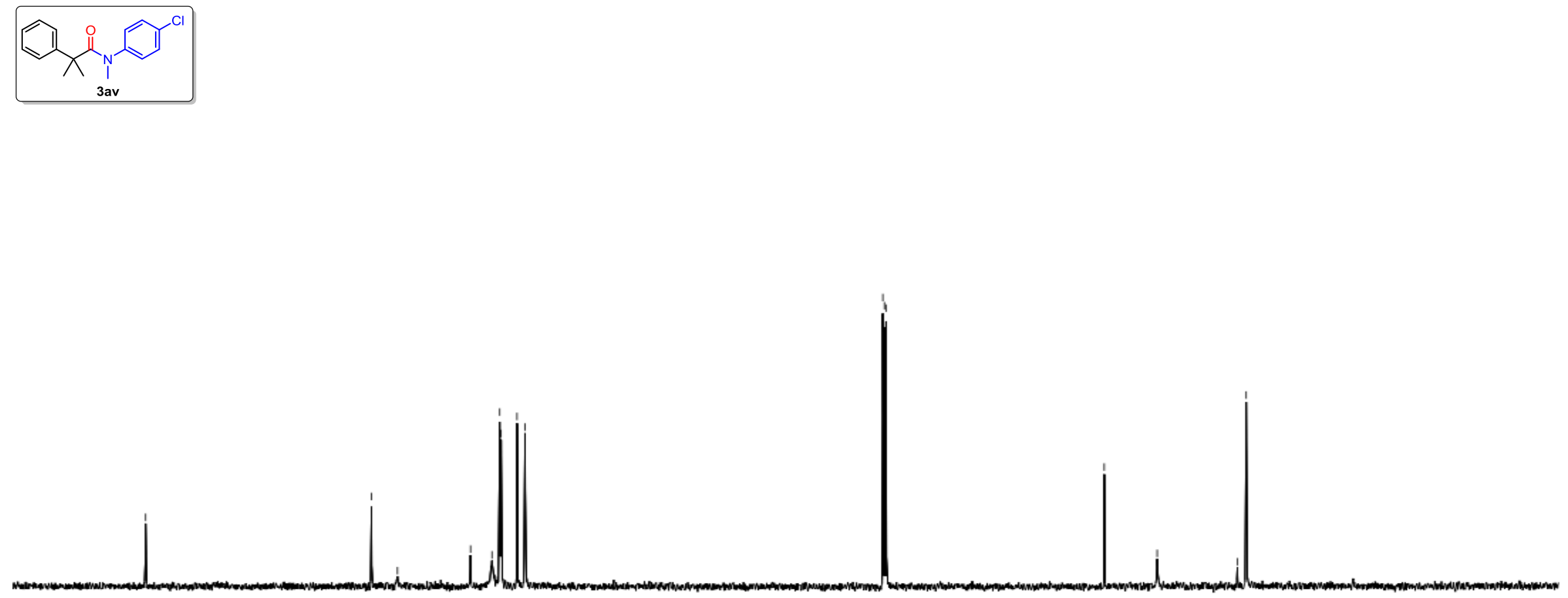

$190 \quad 180$

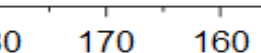

$150 \quad 140$

130

120

110 
${ }^{1} \mathrm{H}$ NMR Spectra (600 MHz, $d^{6}$-DMSO) of compound 3aw

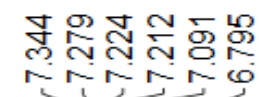

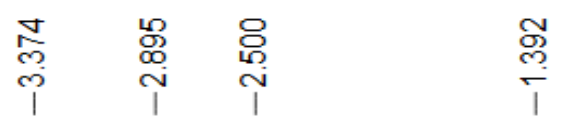
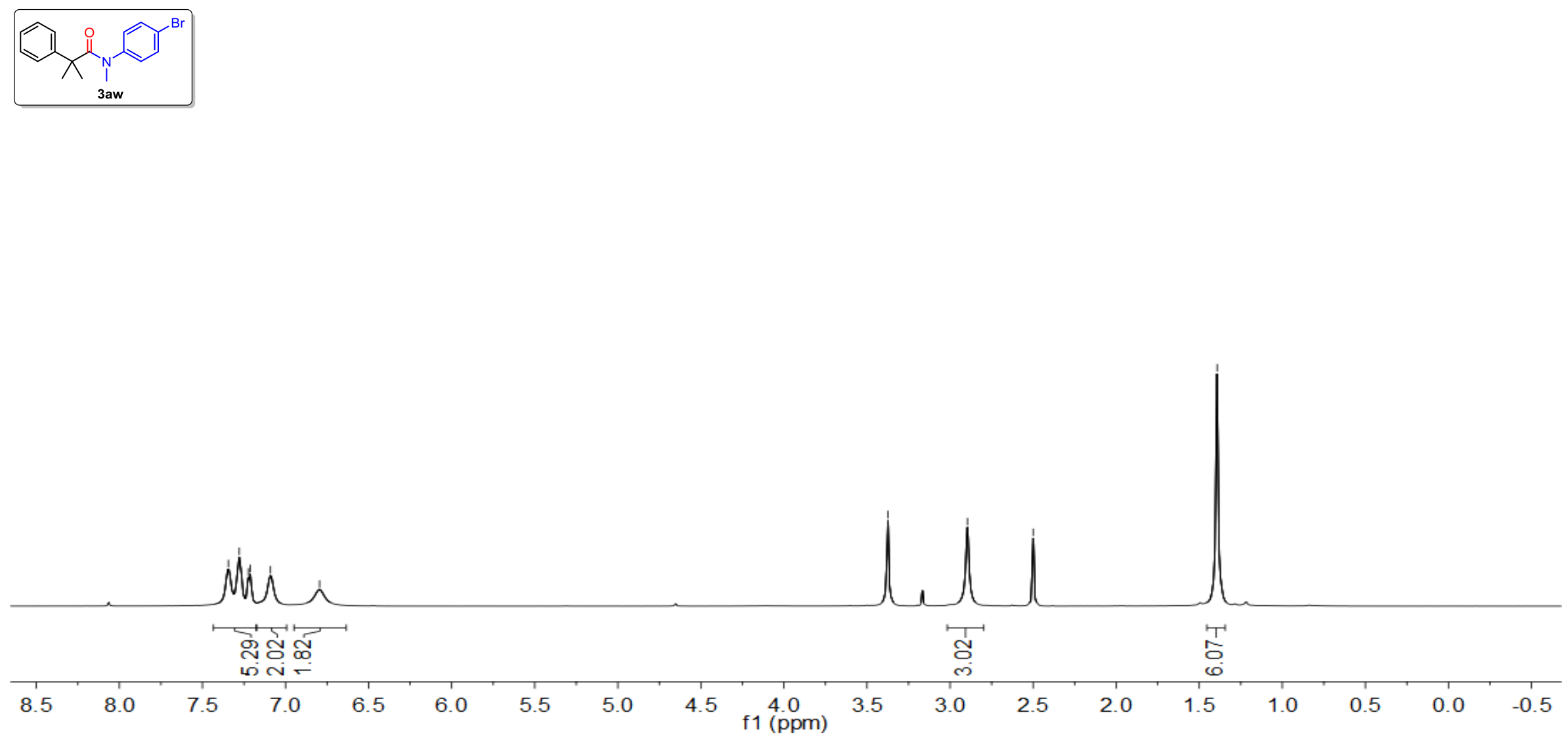
${ }^{13} \mathrm{C}$ NMR Spectra (151 MHz, $d^{6}$-DMSO) of compound 3aw

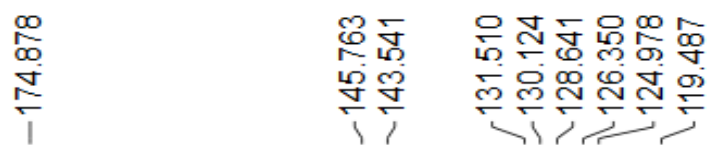

สิ

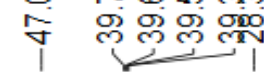
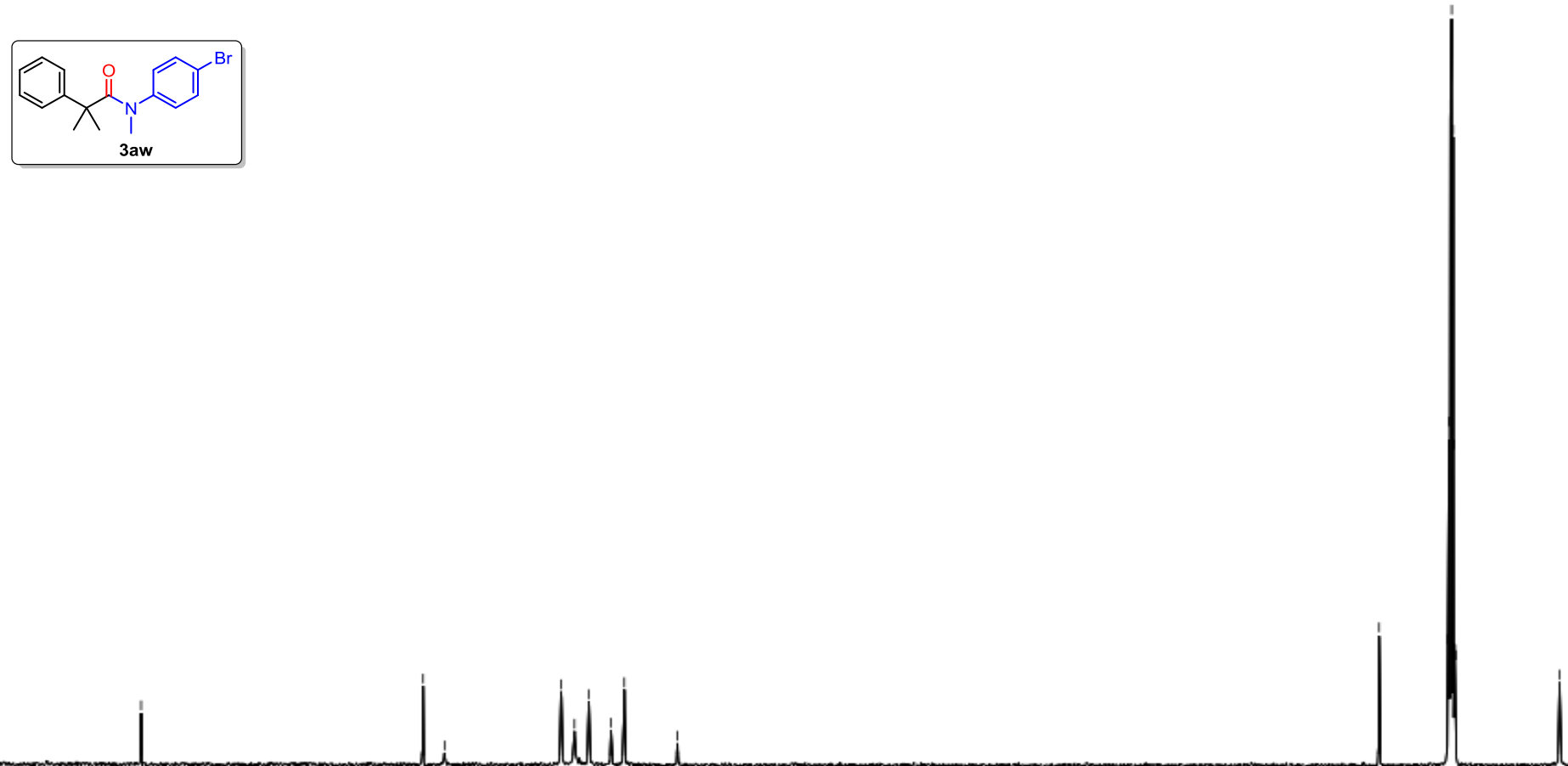

$\begin{array}{lll}190 & 180 & 170\end{array}$

160

$150 \quad 140$

$130 \quad 120$

110

10090

80

70

60

50 40 30

20 
${ }^{1} \mathrm{H}$ NMR Spectra (400 MHz, $\mathrm{CDCl}_{3}$ ) of compound 3ax

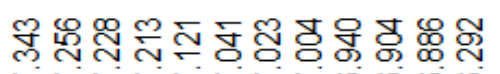

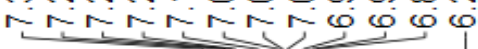
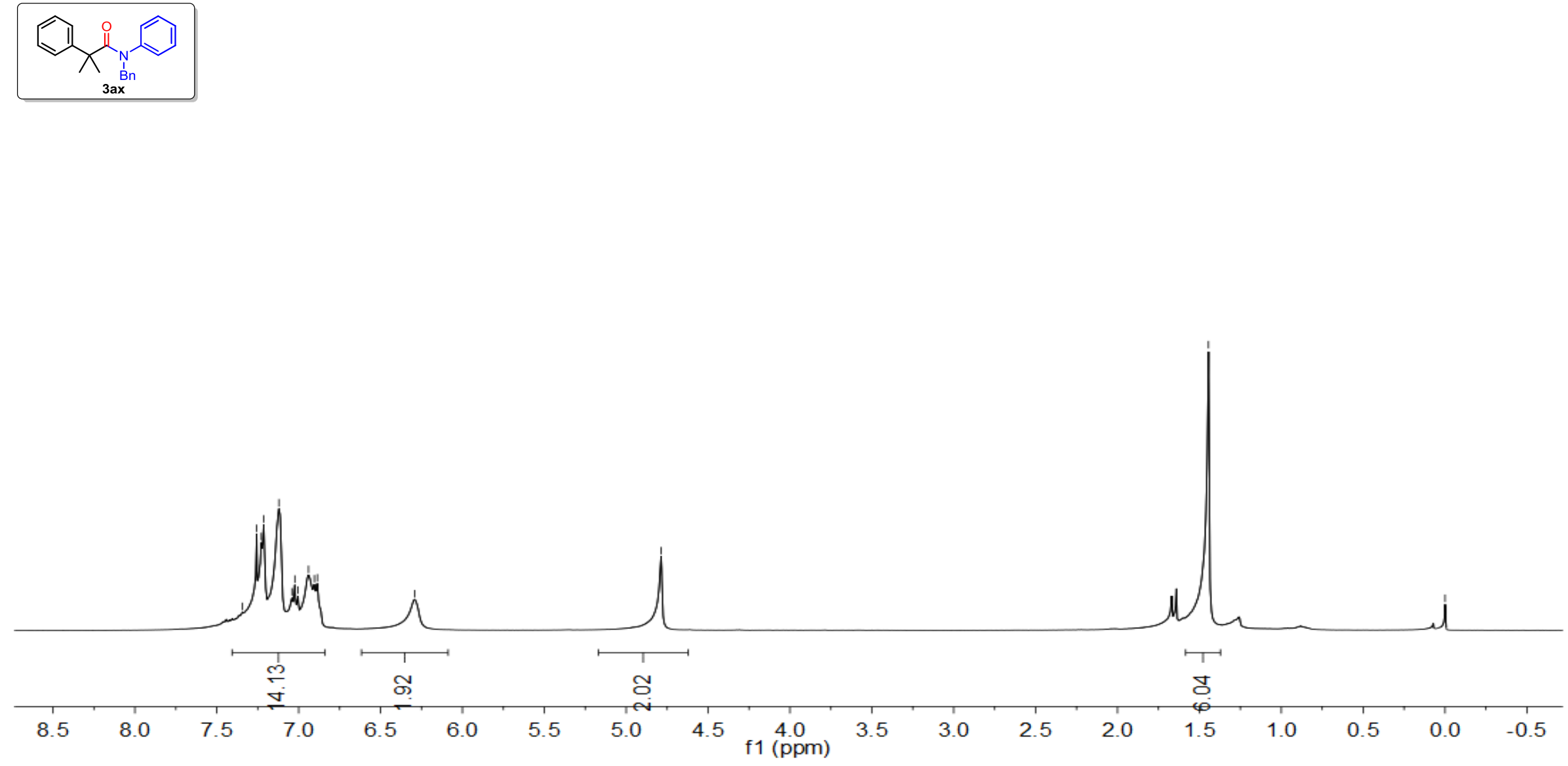
${ }^{13} \mathrm{C}$ NMR Spectra (101 MHz, $\mathrm{CDCl}_{3}$ ) of compound 3ax

옹

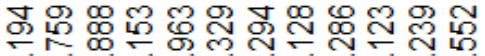

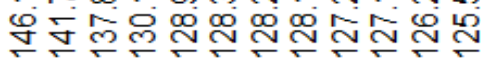

요용

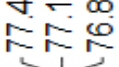

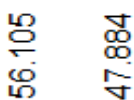

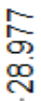
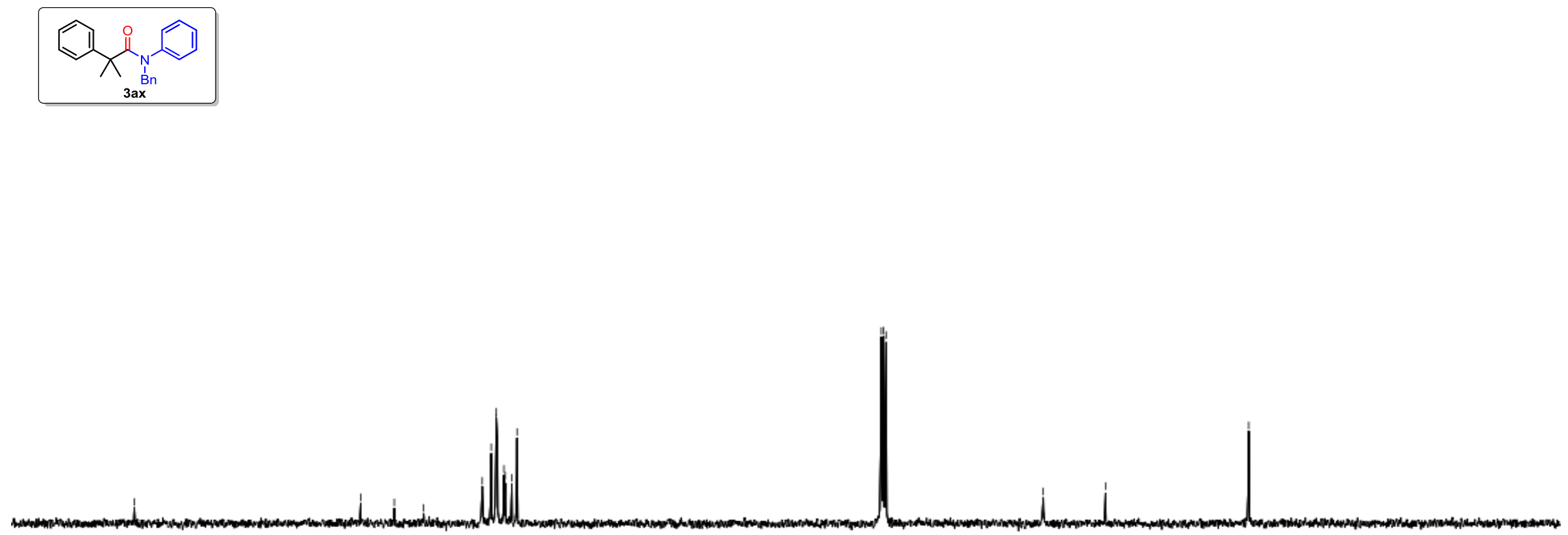

$190 \quad 180$

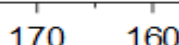

$160 \quad 150$

140 
${ }^{1} \mathrm{H}$ NMR Spectra (400 MHz, $\mathrm{CDCl}_{3}$ ) of compound 3ay

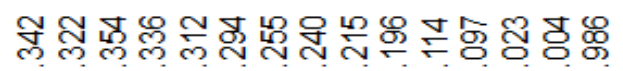

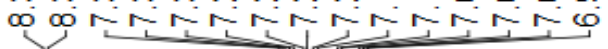

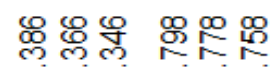

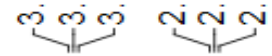
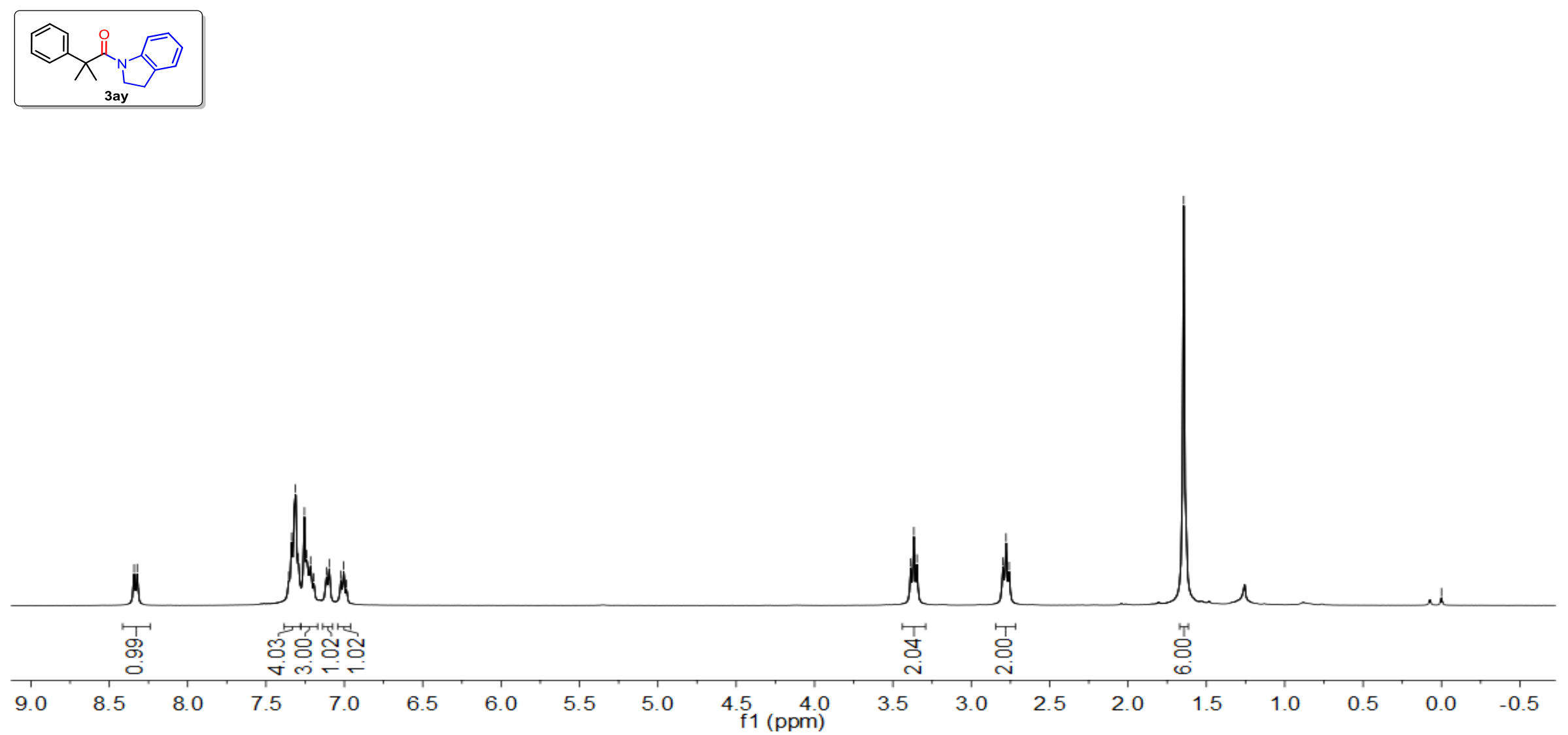
${ }^{13} \mathrm{C}$ NMR Spectra (101 MHz, $\mathrm{CDCl}_{3}$ ) of compound 3ay
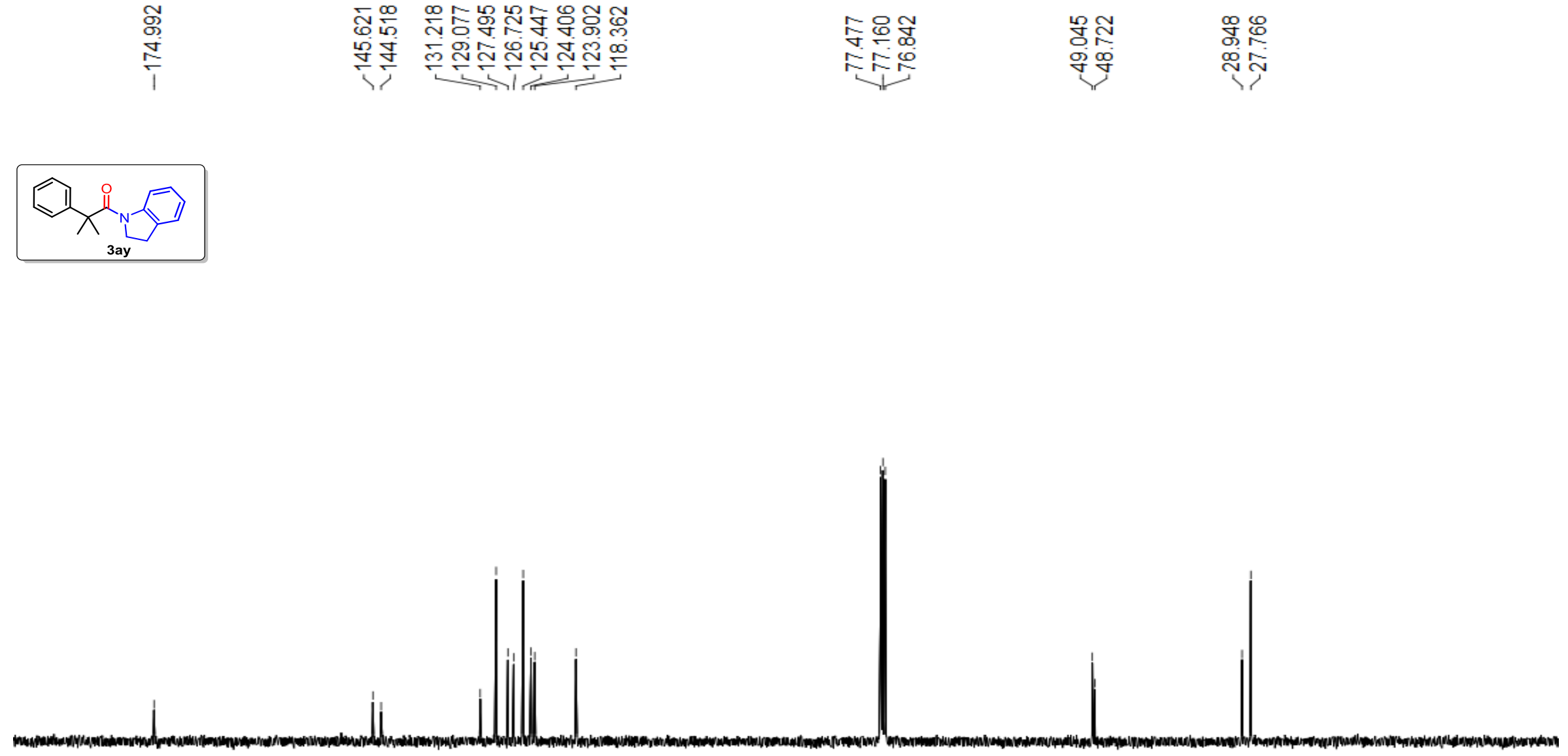

$190 \quad 180 \quad 170 \quad 160$

150

140

130

120

$100 \quad 90$

80

70

60

50

40

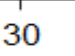

20

$0 \quad-10$ 
${ }^{1} \mathrm{H}$ NMR Spectra (400 MHz, $\mathrm{CDCl}_{3}$ ) of compound $\mathbf{3 b a}$

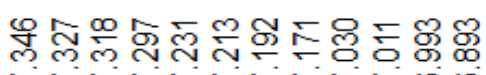

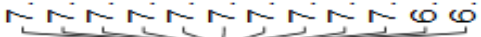

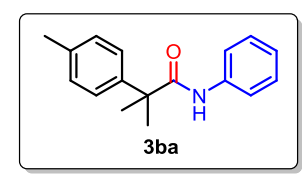

$\stackrel{\text { i }}{\stackrel{1}{*}}$

吕

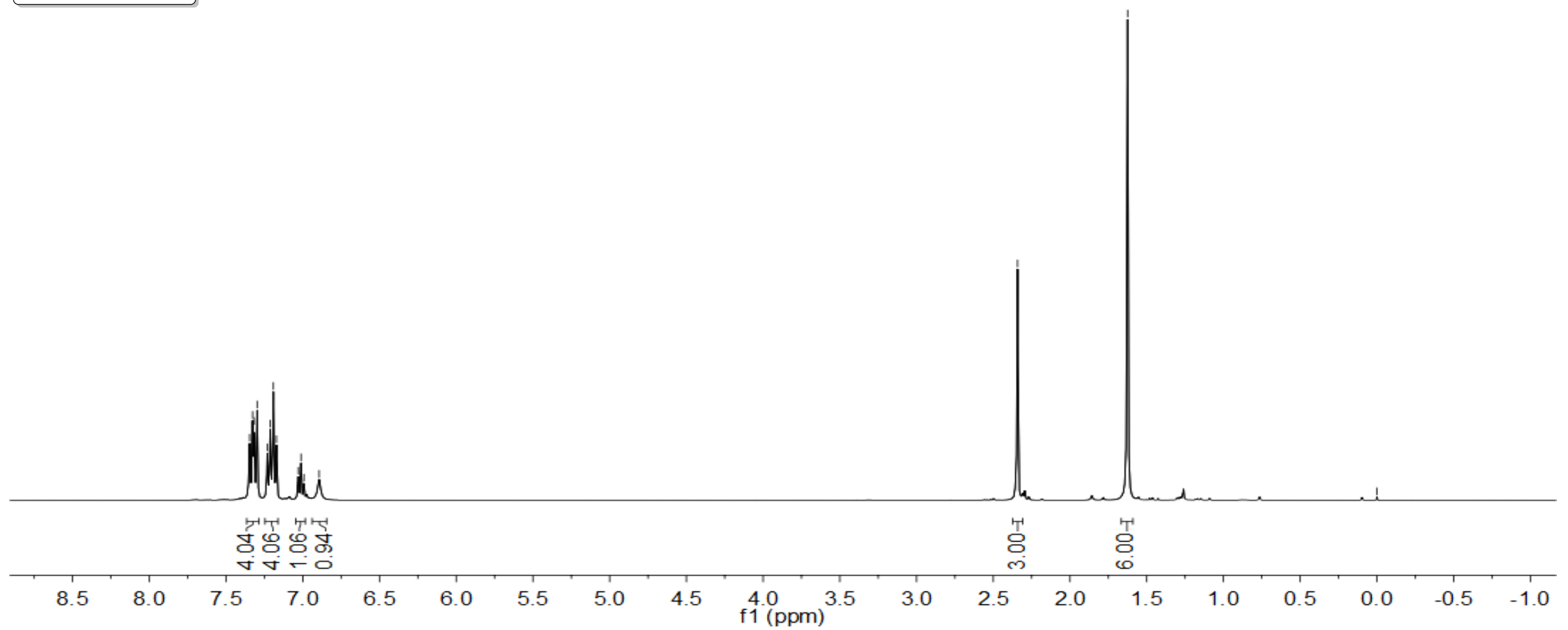


${ }^{13} \mathrm{C}$ NMR Spectra (101 MHz, $\mathrm{CDCl}_{3}$ ) of compound 3ba

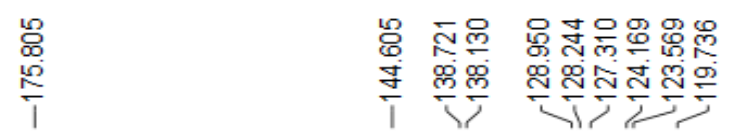

寺

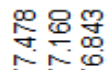

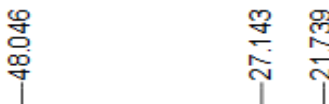
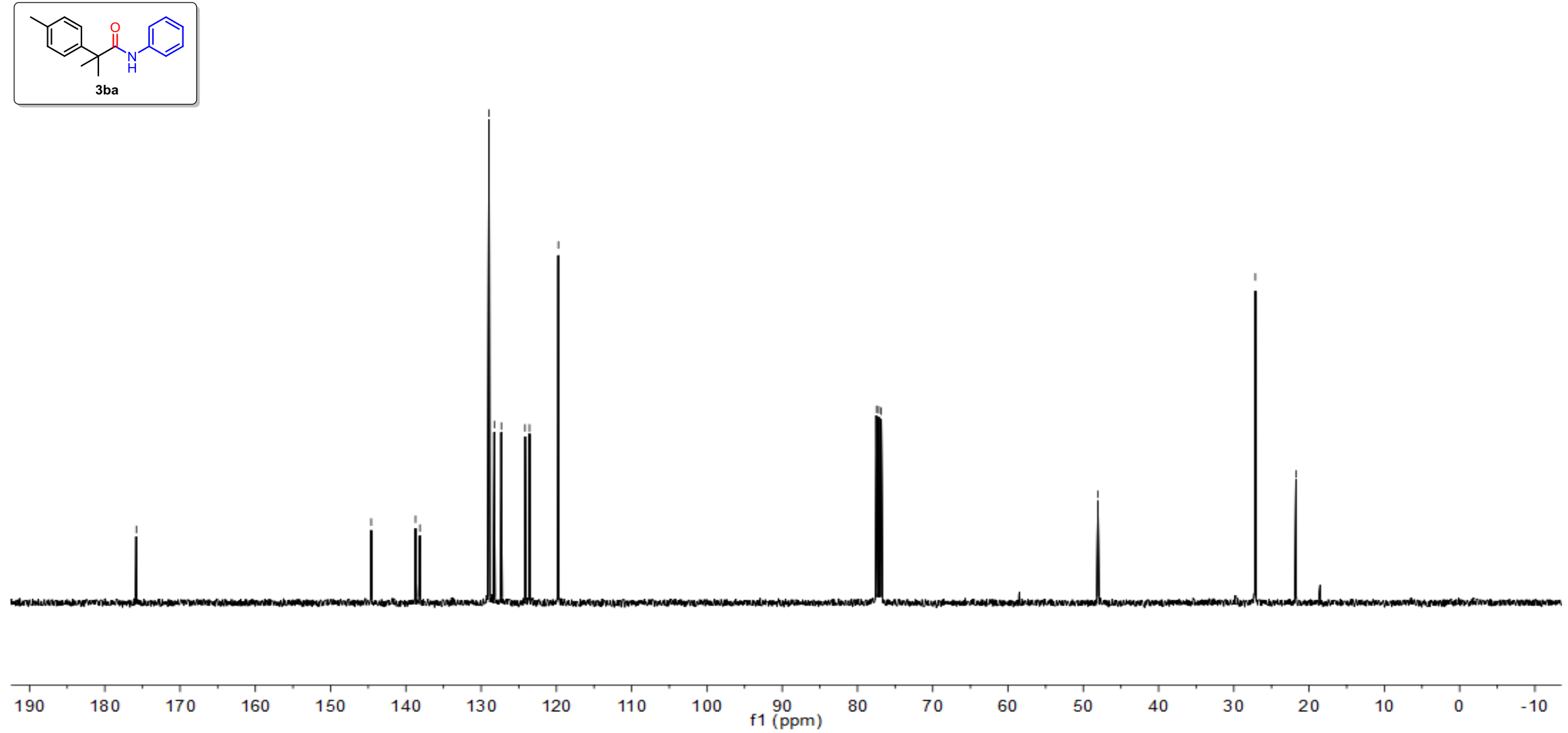
${ }^{1} \mathrm{H}$ NMR Spectra (400 MHz, $\mathrm{CDCl}_{3}$ ) of compound 3ca

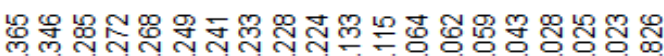

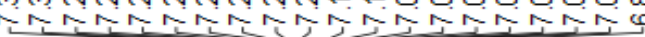

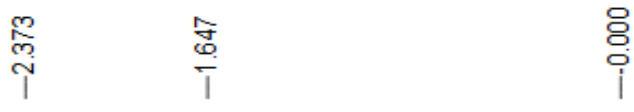
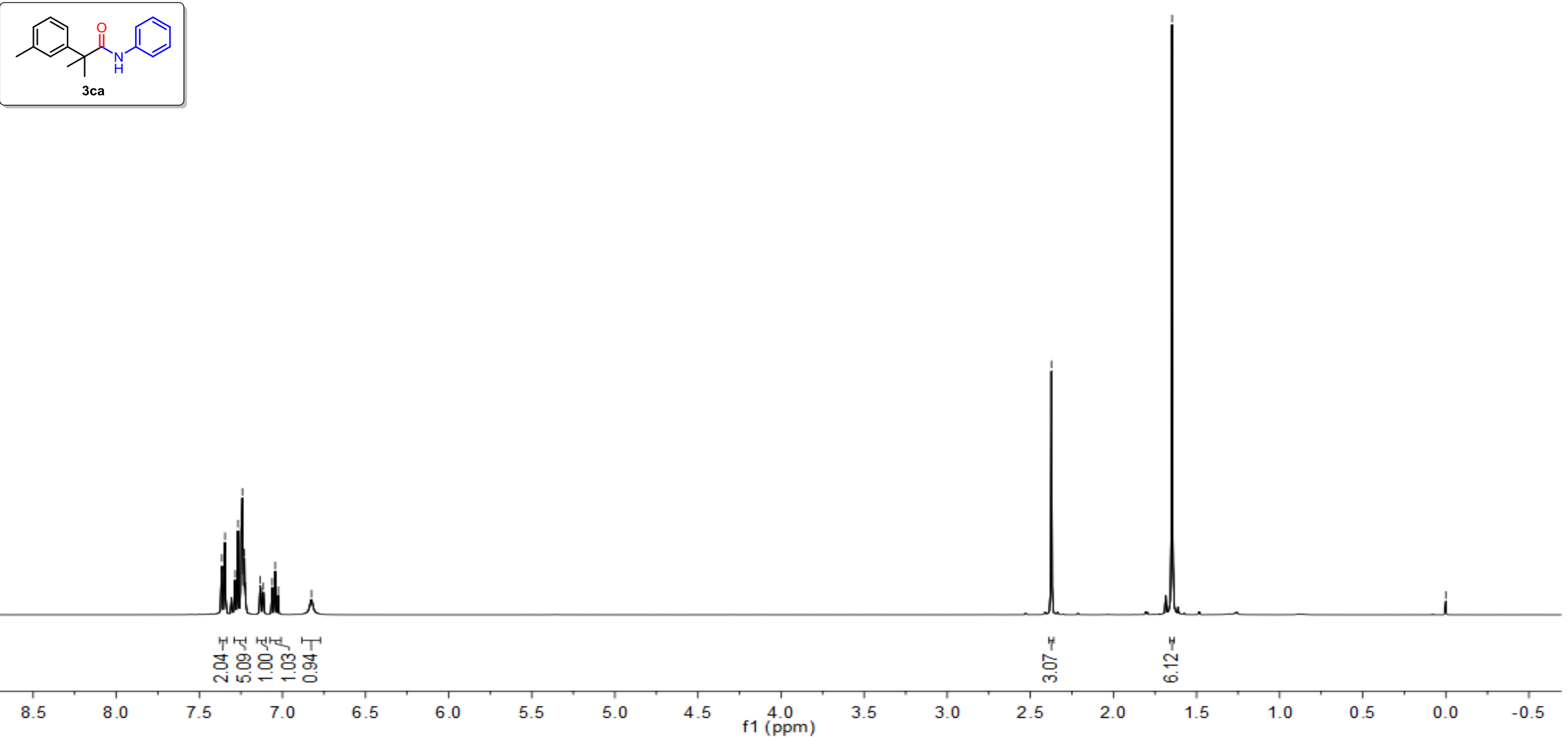
${ }^{13} \mathrm{C}$ NMR Spectra (101 MHz, $\mathrm{CDCl}_{3}$ ) of compound 3ca

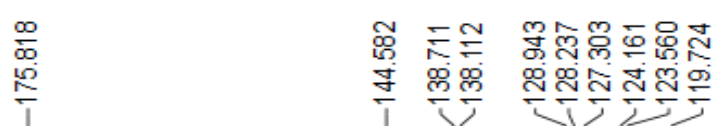

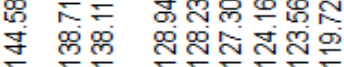

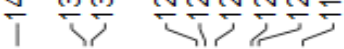

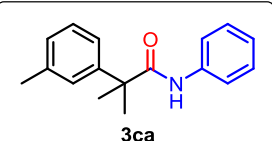

3ca

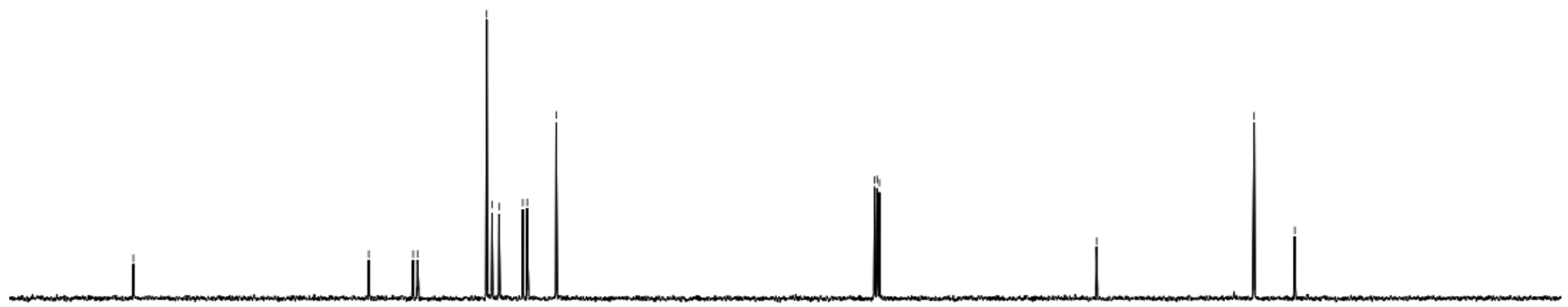


${ }^{1} \mathrm{H}$ NMR Spectra (400 MHz, $\mathrm{CDCl}_{3}$ ) of compound 3ca

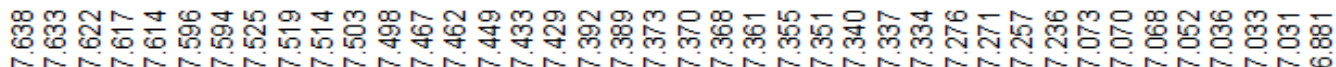
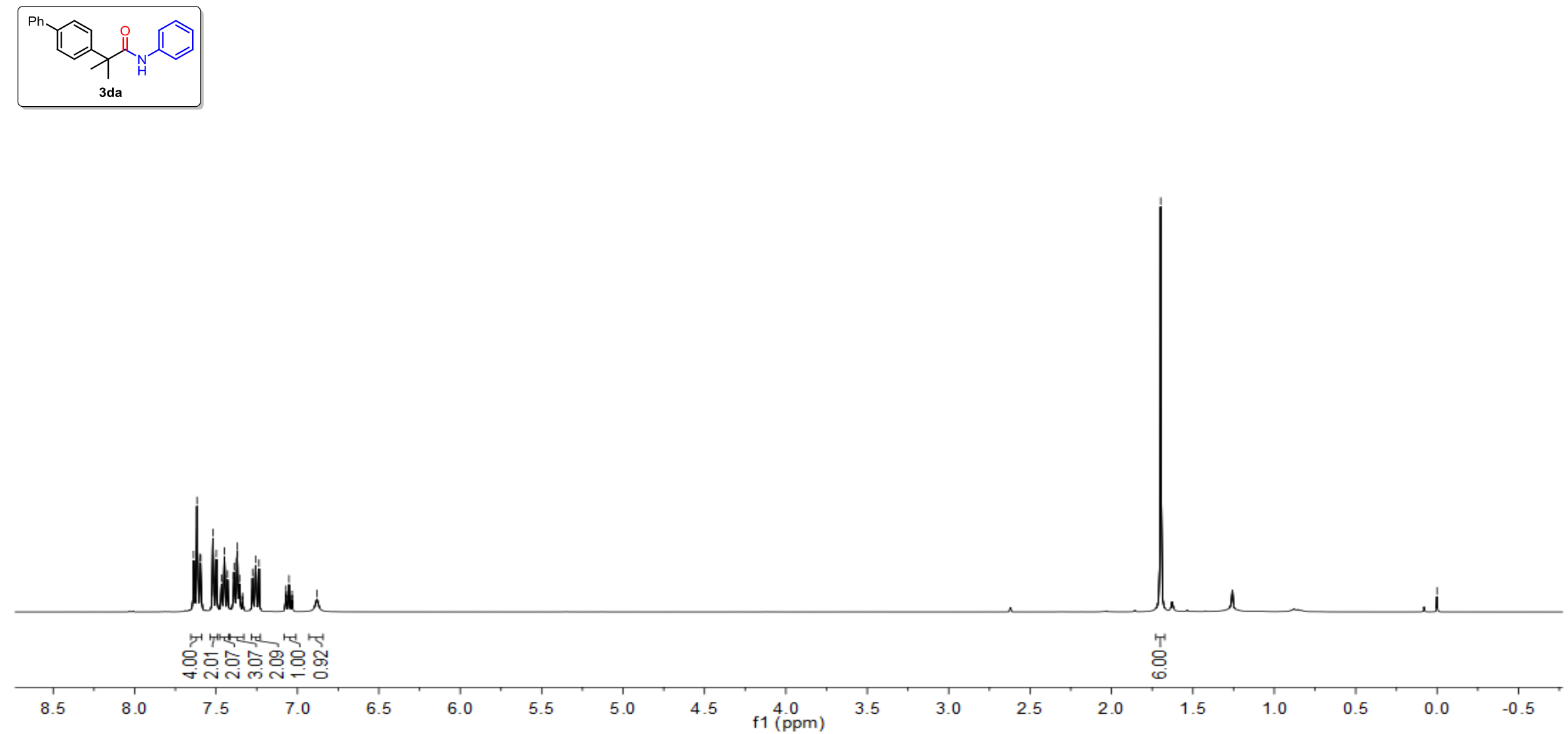
${ }^{13} \mathrm{C}$ NMR Spectra (101 MHz, $\mathrm{CDCl}_{3}$ ) of compound 3da

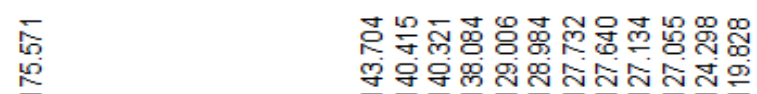

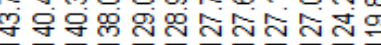
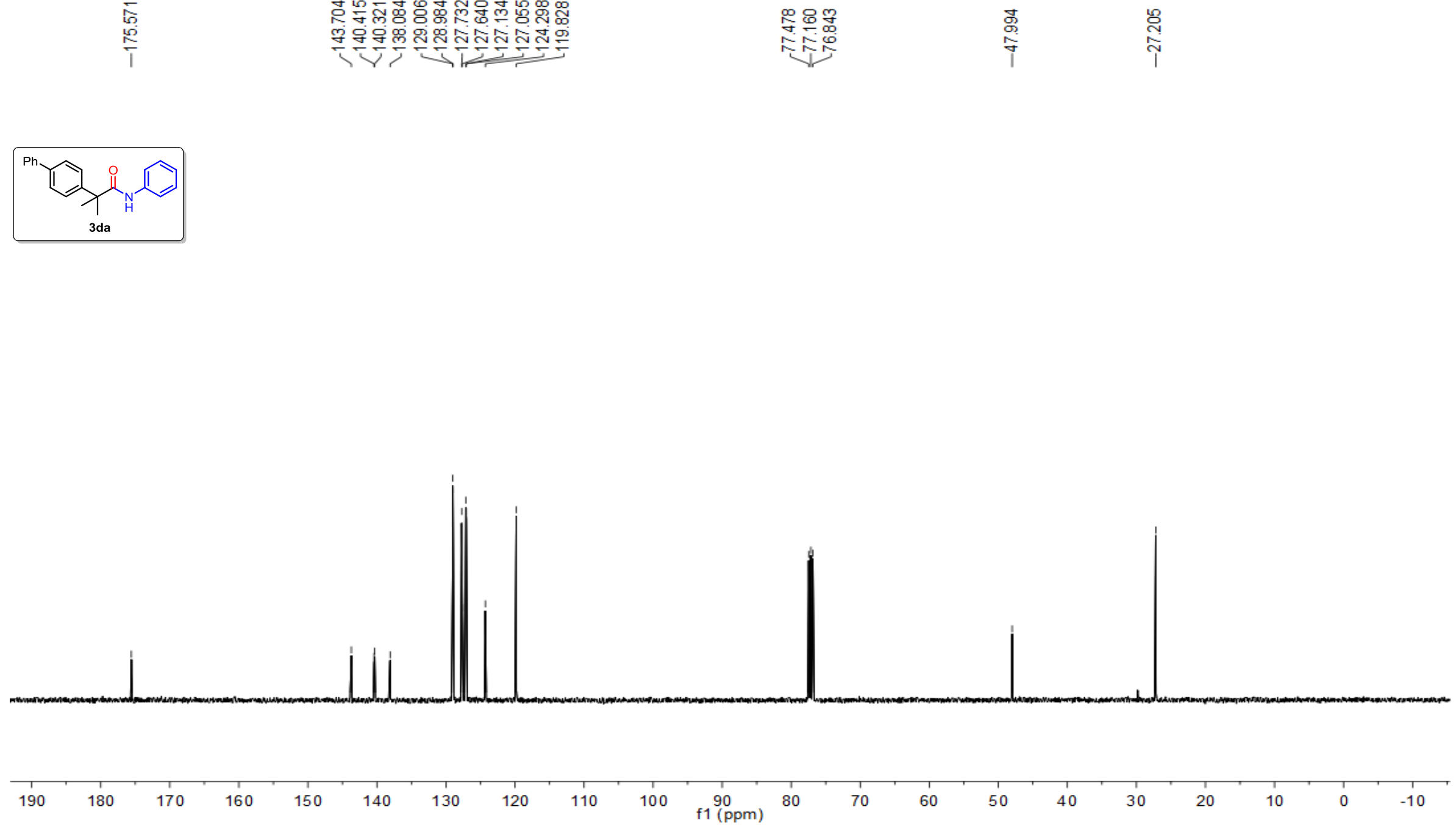
${ }^{1} \mathrm{H}$ NMR Spectra (400 MHz, $\mathrm{CDCl}_{3}$ ) of compound 3ea

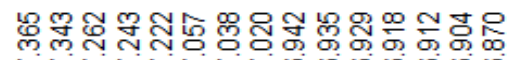

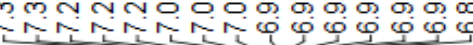
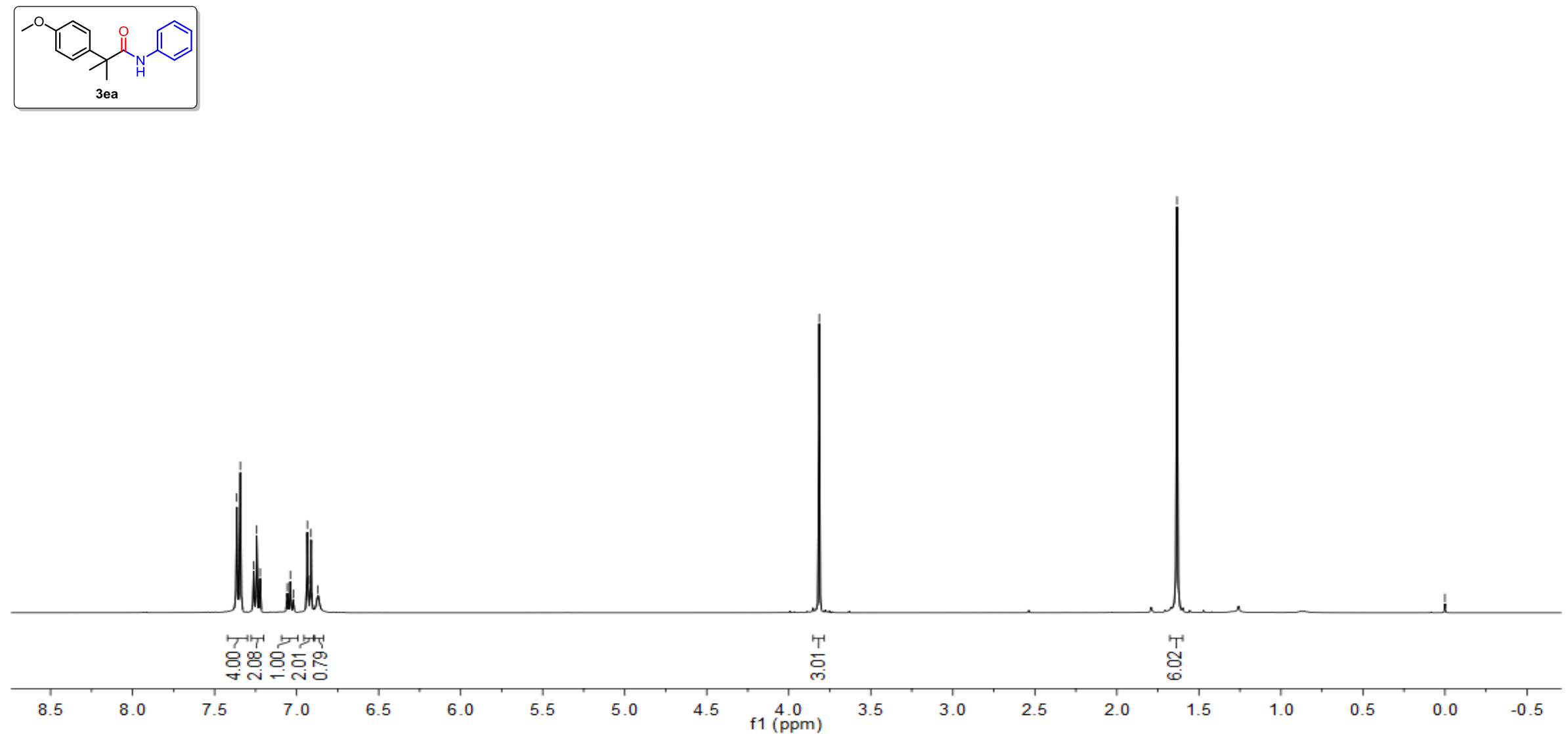
${ }^{13} \mathrm{C}$ NMR Spectra (101 MHz, $\mathrm{CDCl}_{3}$ ) of compound 3ea

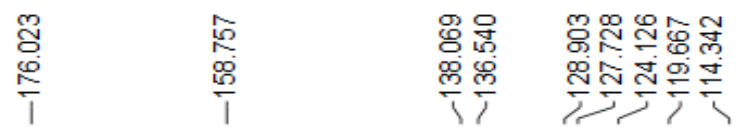

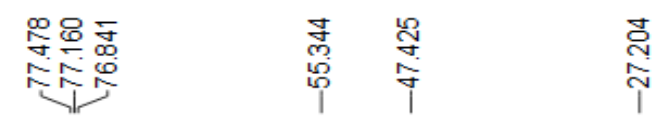
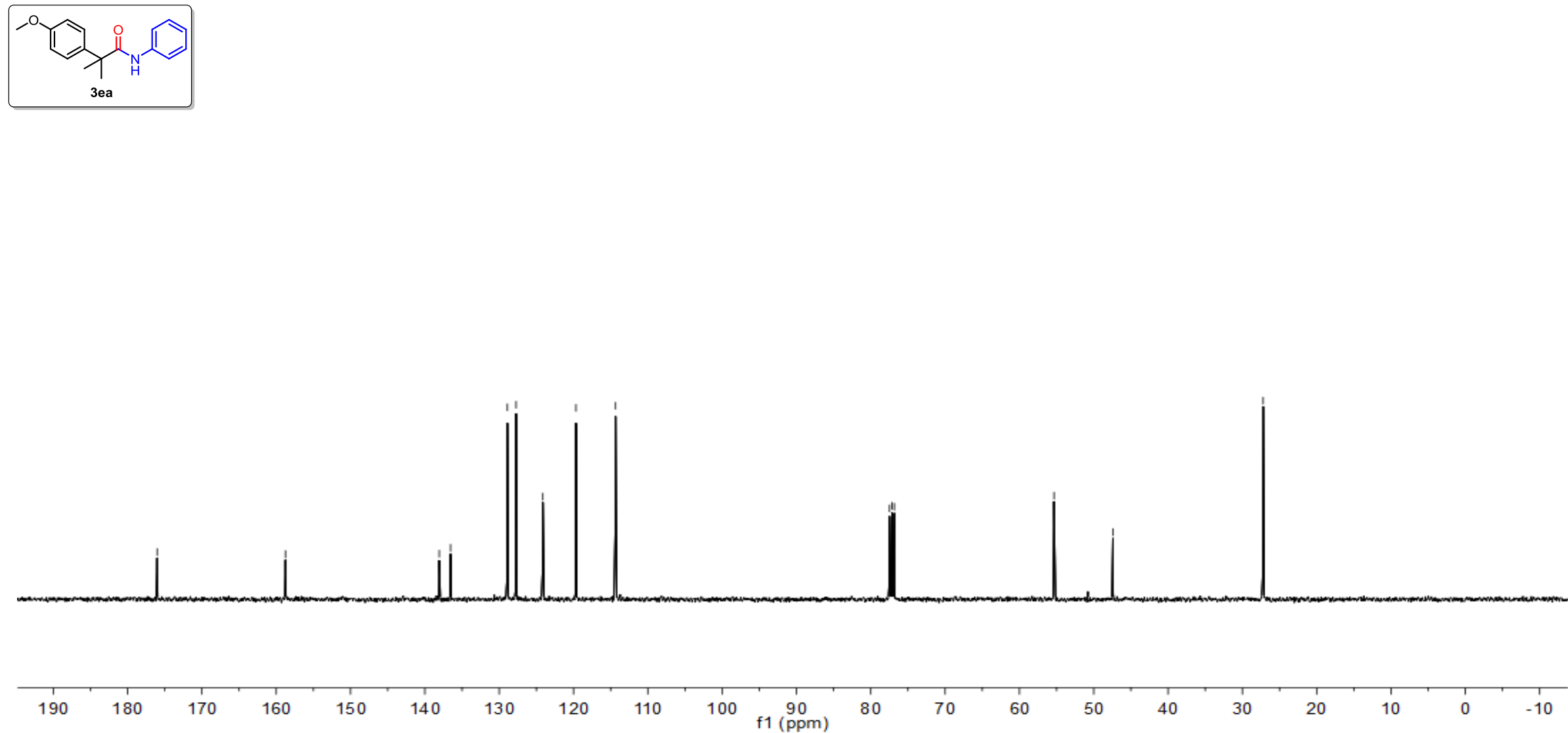
${ }^{1} \mathrm{H}$ NMR Spectra (400 MHz, $\mathrm{CDCl}_{3}$ ) of compound 3fa

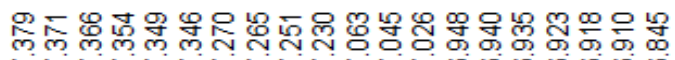

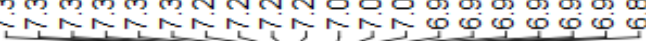
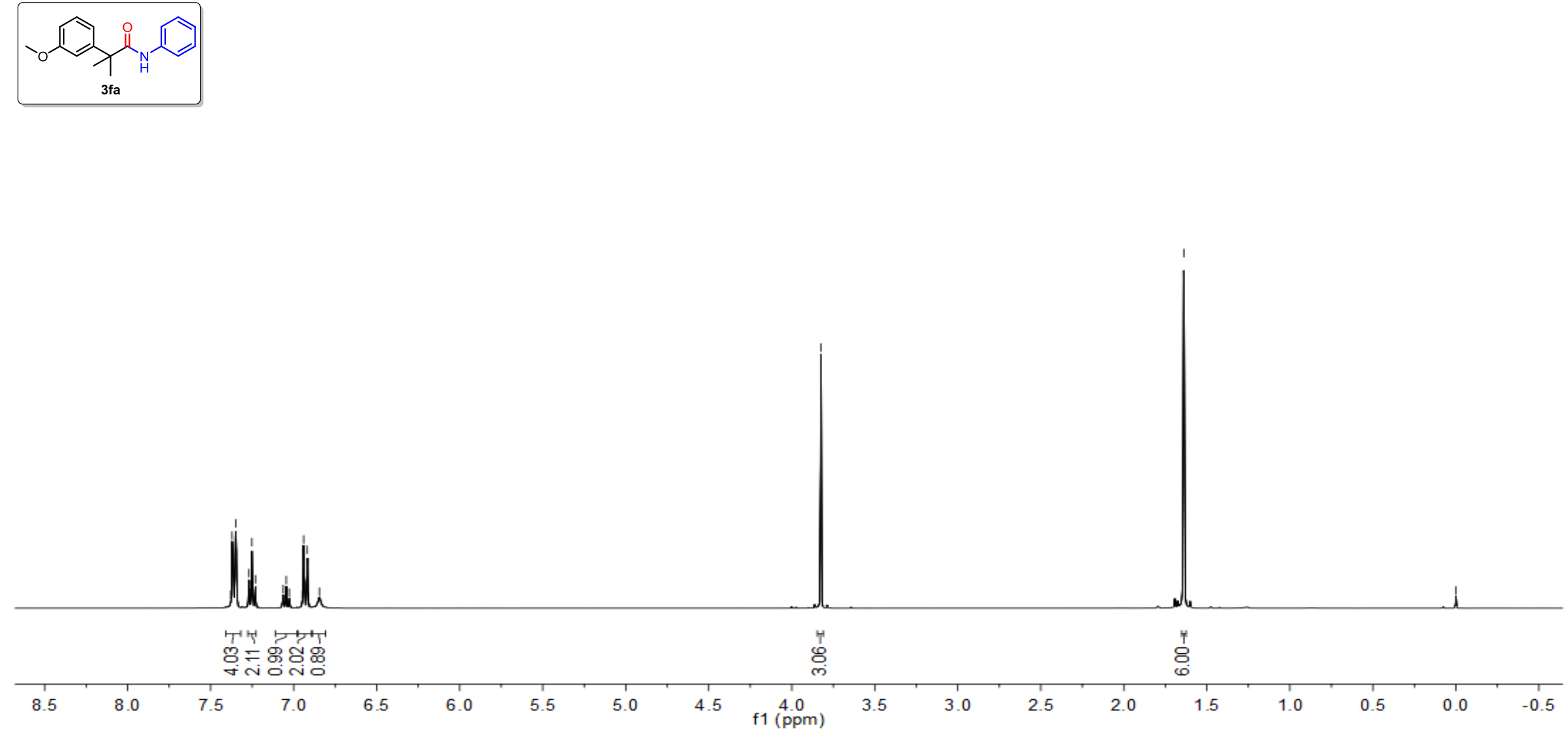
${ }^{13} \mathrm{C}$ NMR Spectra (101 MHz, $\mathrm{CDCl}_{3}$ ) of compound $\mathbf{3 f a}$

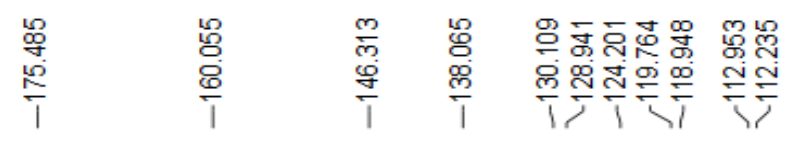

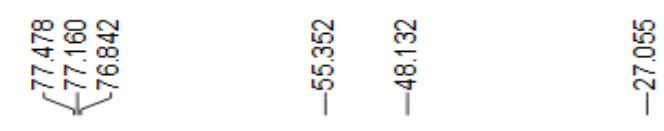
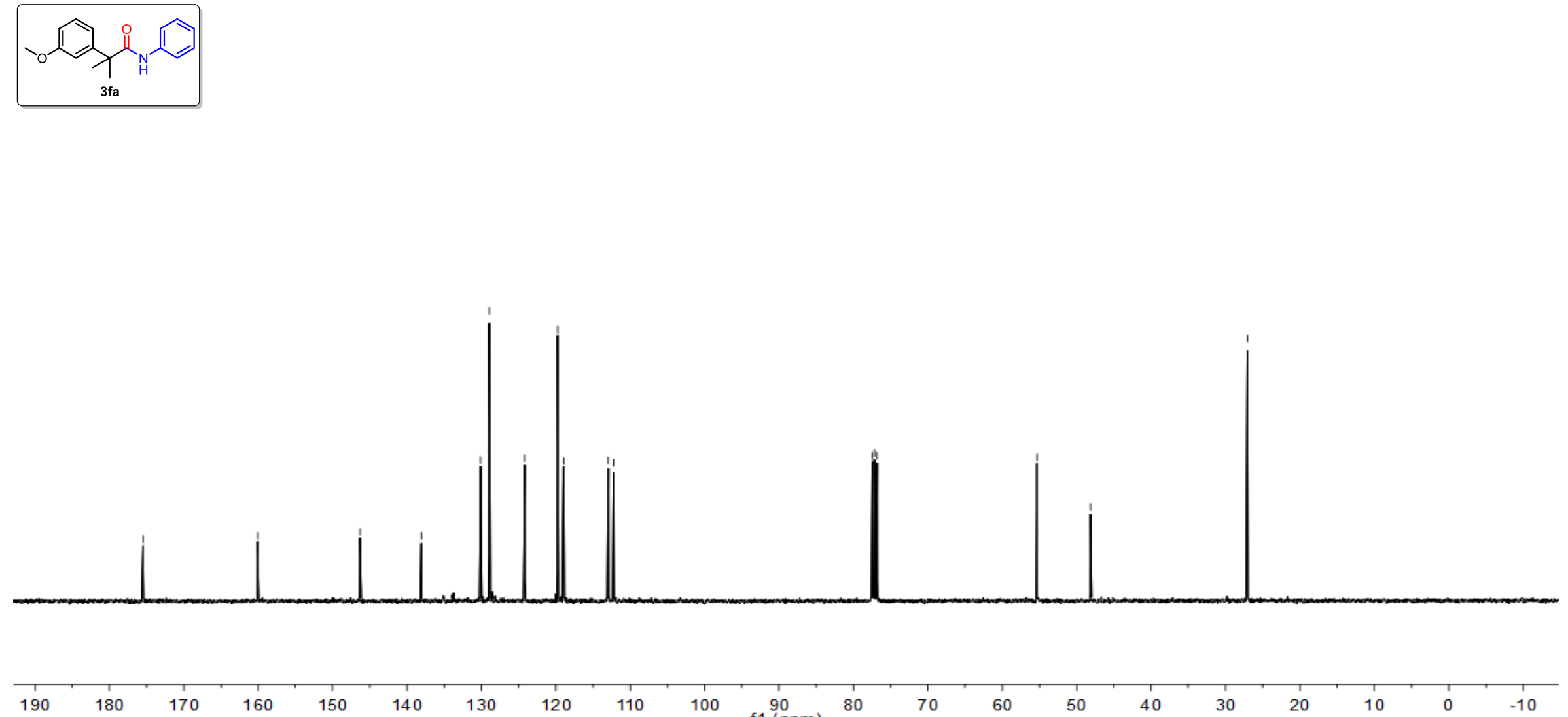

160

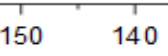

130

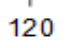

110

100

f1 $\stackrel{90}{(\mathrm{ppm})}$
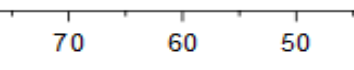
${ }^{1} \mathrm{H}$ NMR Spectra (400 MHz, $\mathrm{CDCl}_{3}$ ) of compound 3ga

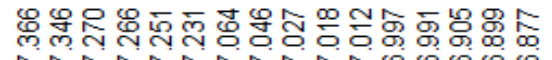

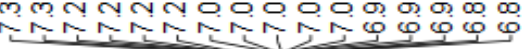
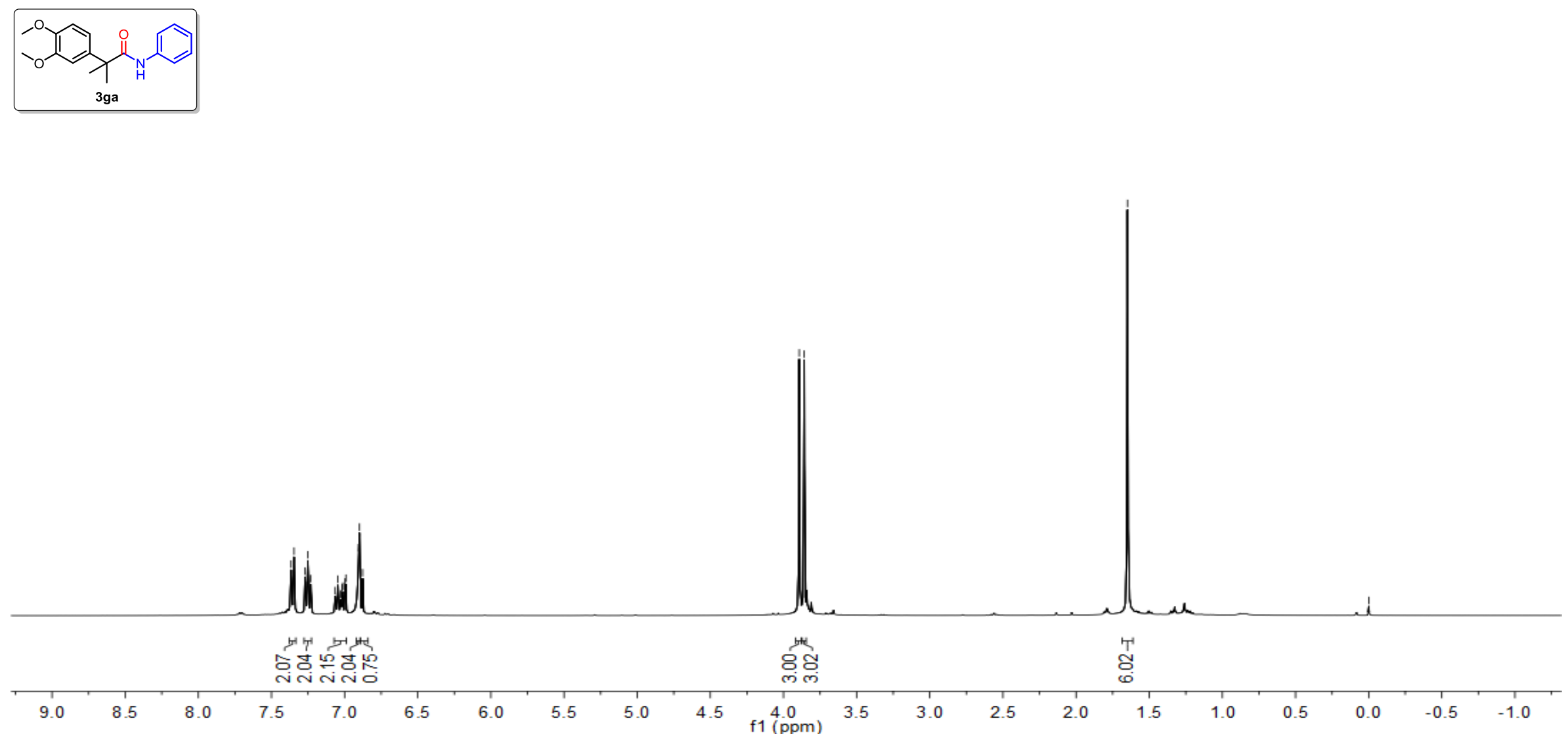
${ }^{13} \mathrm{C}$ NMR Spectra (101 MHz, $\mathrm{CDCl}_{3}$ ) of compound 3ga
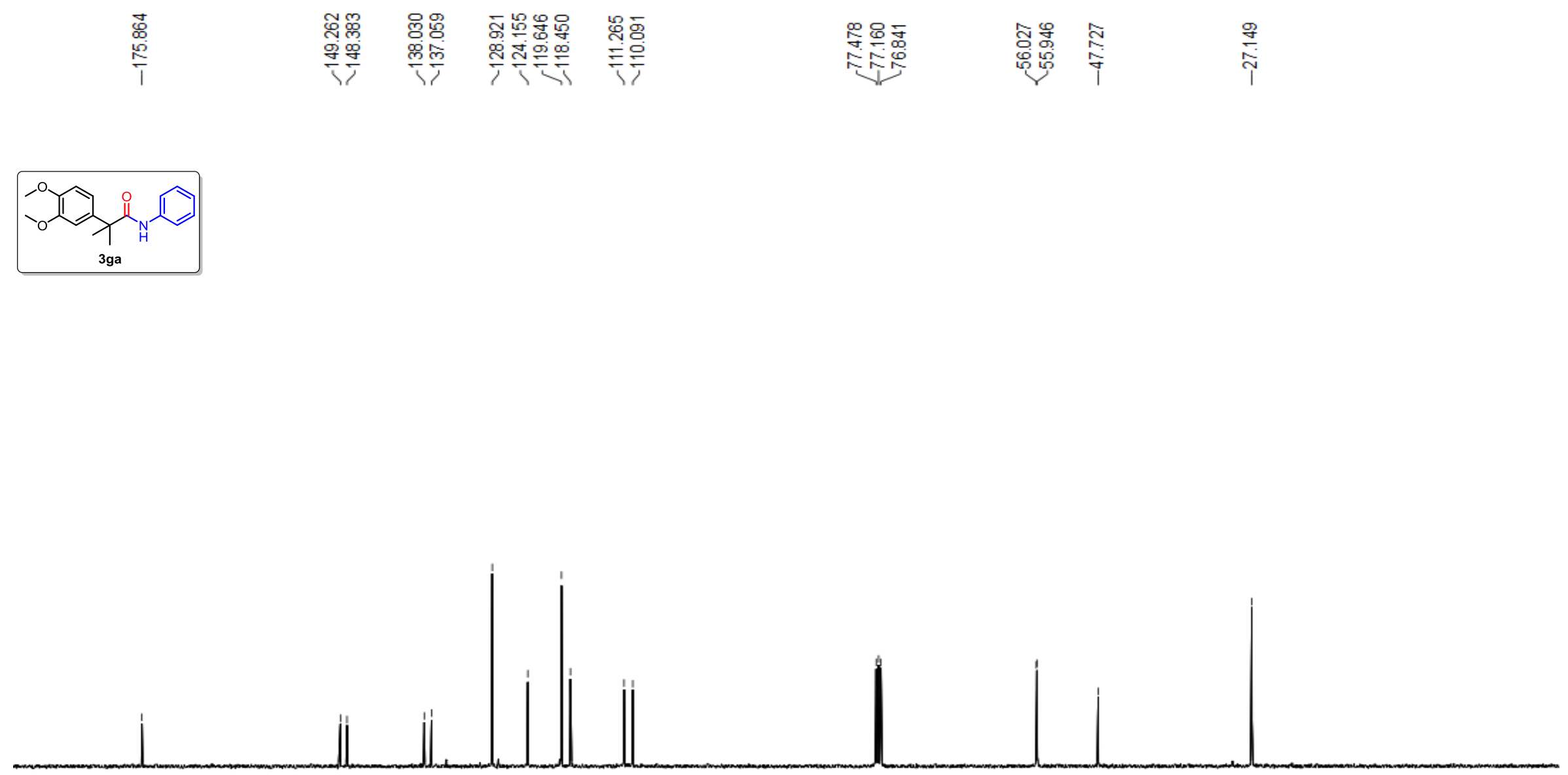
${ }^{1} \mathrm{H}$ NMR Spectra (400 MHz, $\mathrm{CDCl}_{3}$ ) of compound 3ha

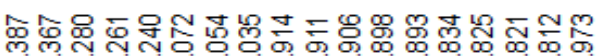

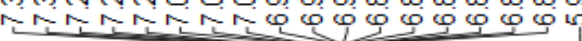

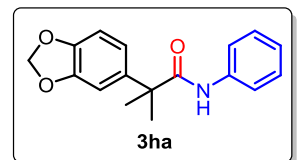

3ha

i⿱

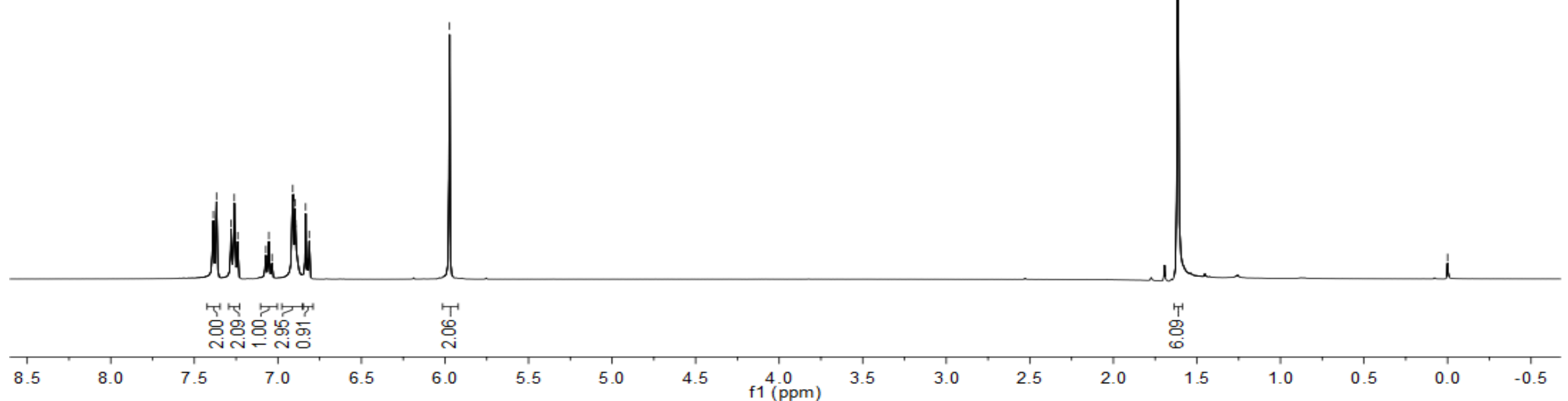


${ }^{13} \mathrm{C}$ NMR Spectra (101 MHz, $\mathrm{CDCl}_{3}$ ) of compound 3ha

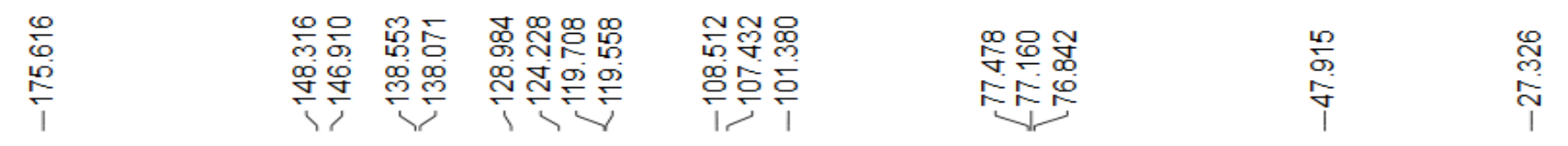
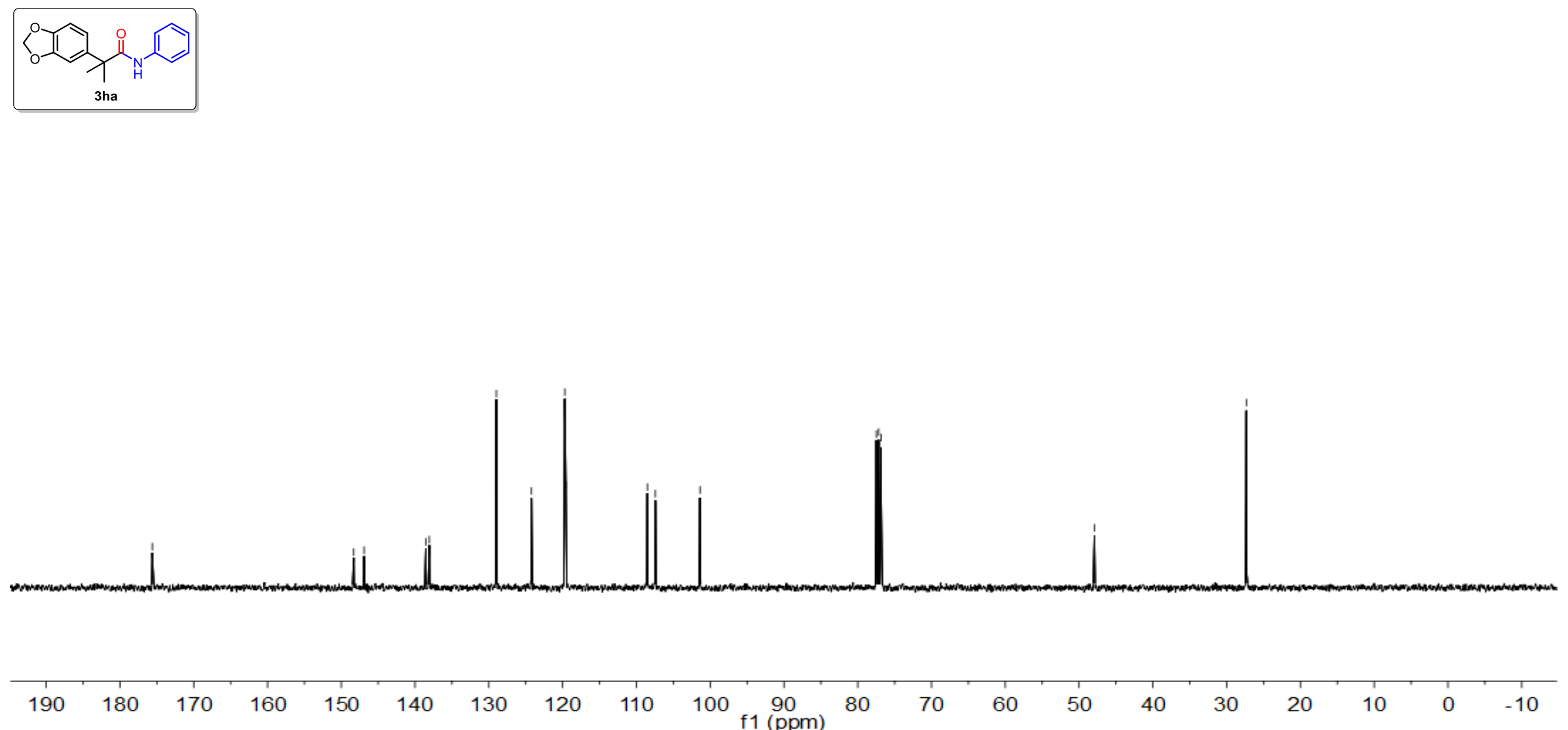
${ }^{1} \mathrm{H}$ NMR Spectra (400 MHz, $\mathrm{CDCl}_{3}$ ) of compound 3ia

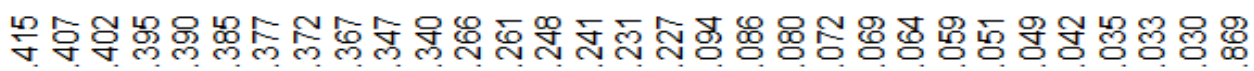

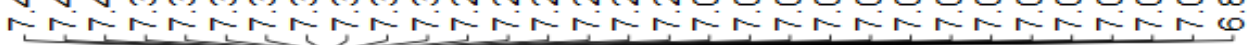
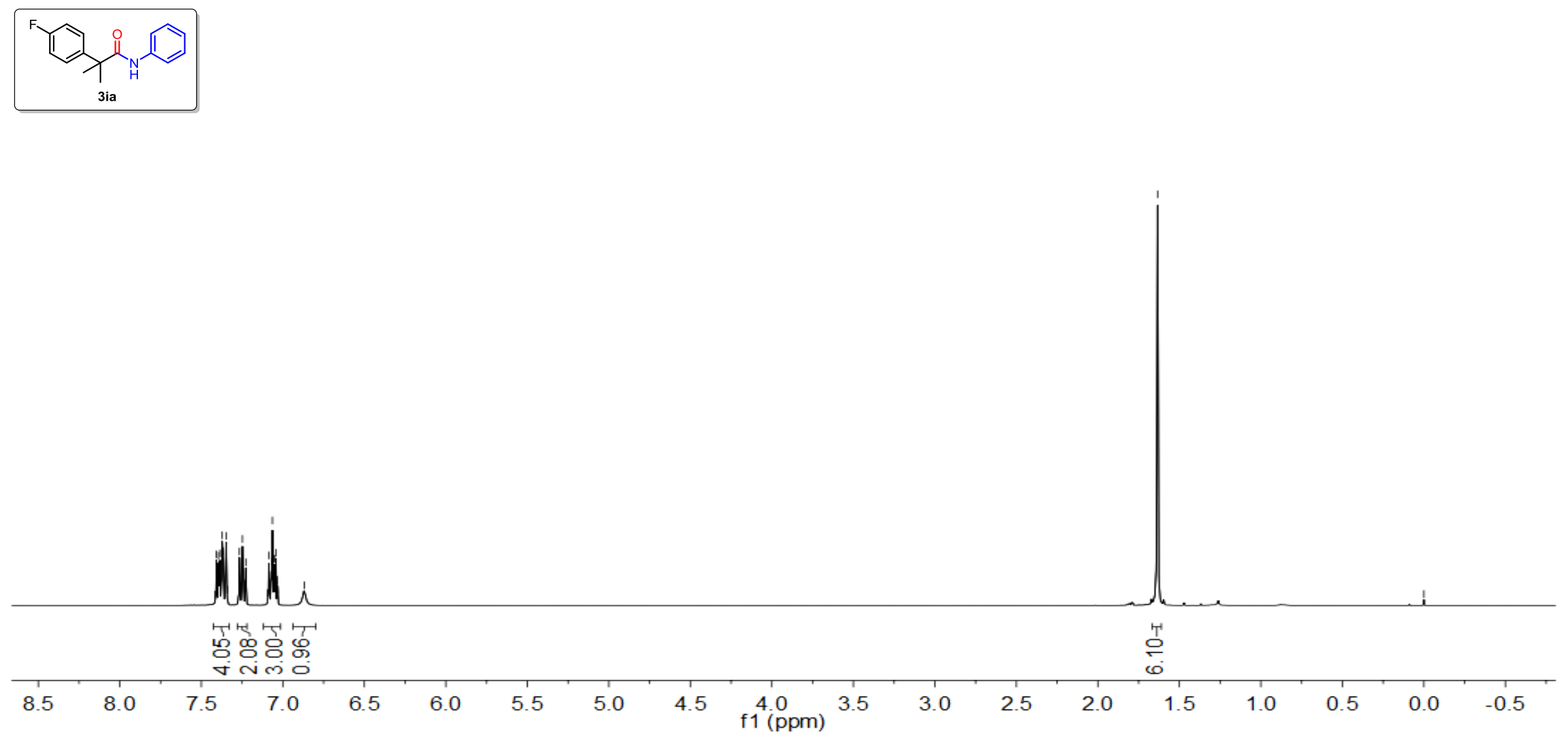
${ }^{13} \mathrm{C}$ NMR Spectra (101 MHz, $\mathrm{CDCl}_{3}$ ) of compound 3ia

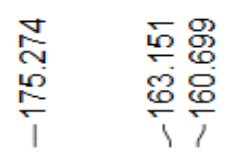

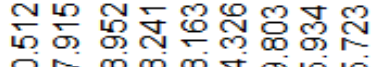

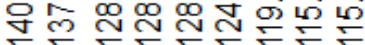

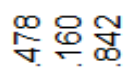

숭

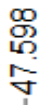

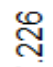

V

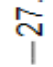
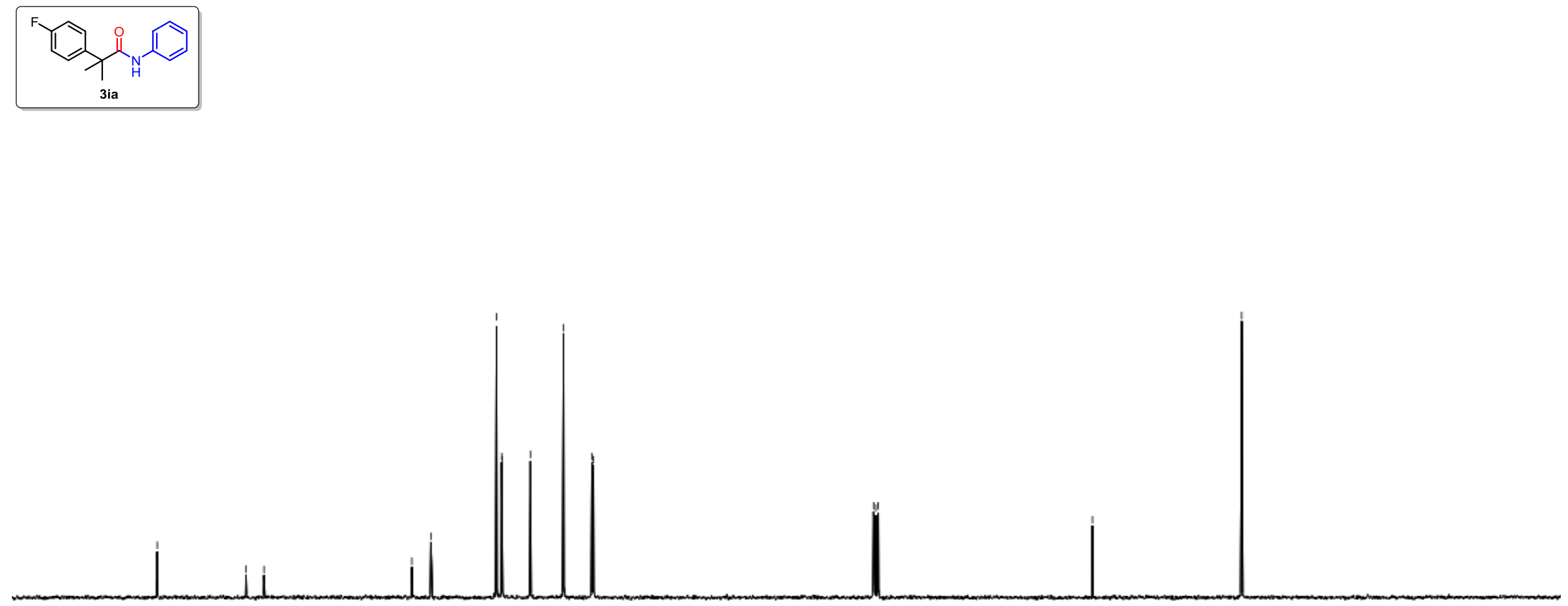

$190 \quad 180$

$170 \quad 160$

150140

130

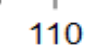

$100 \quad 90$

90

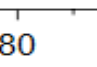

70

60

50

40

$30 \quad 20$ 
${ }^{19} \mathrm{~F}$ NMR Spectra $\left(375 \mathrm{MHz}, \mathrm{CDCl}_{3}\right.$ ) of compound 3ia

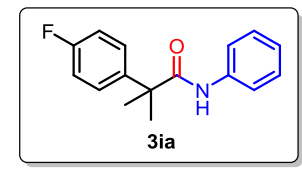

$80 \quad 70 \quad 60$

40

$20 \quad 10$

$10 \quad 0$

$-10 \quad-20$

$-20 \quad-30$

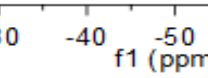

$-\frac{1}{-50}-60$

S108 
${ }^{1} \mathrm{H}$ NMR Spectra $\left(400 \mathrm{MHz}, \mathrm{CDCl}_{3}\right)$ of compound $\mathbf{3 j a}$

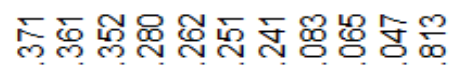

rrrñN-N
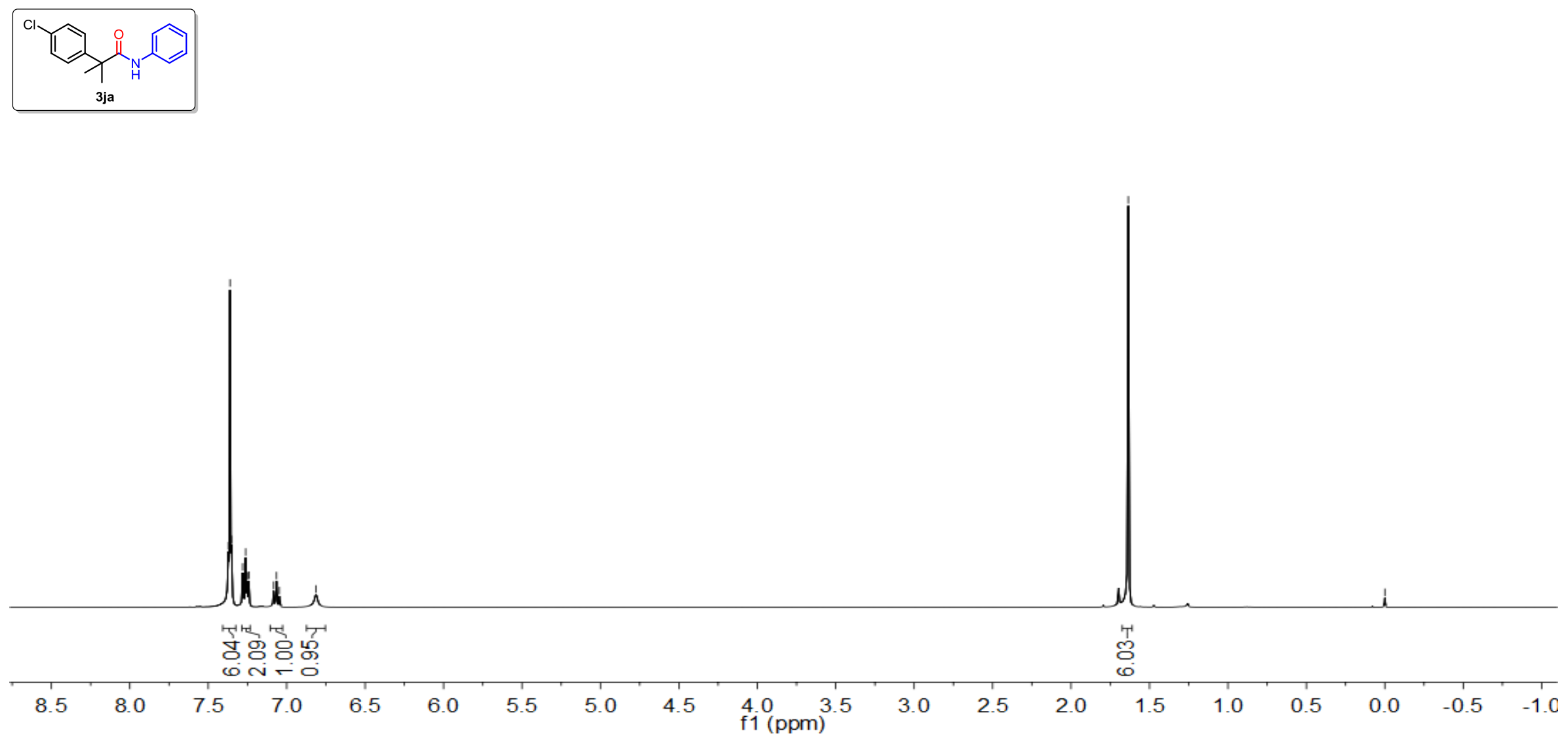
${ }^{13} \mathrm{C}$ NMR Spectra (101 MHz, $\mathrm{CDCl}_{3}$ ) of compound $\mathbf{3 j a}$

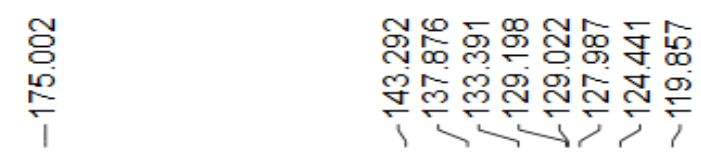

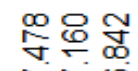

숭

告
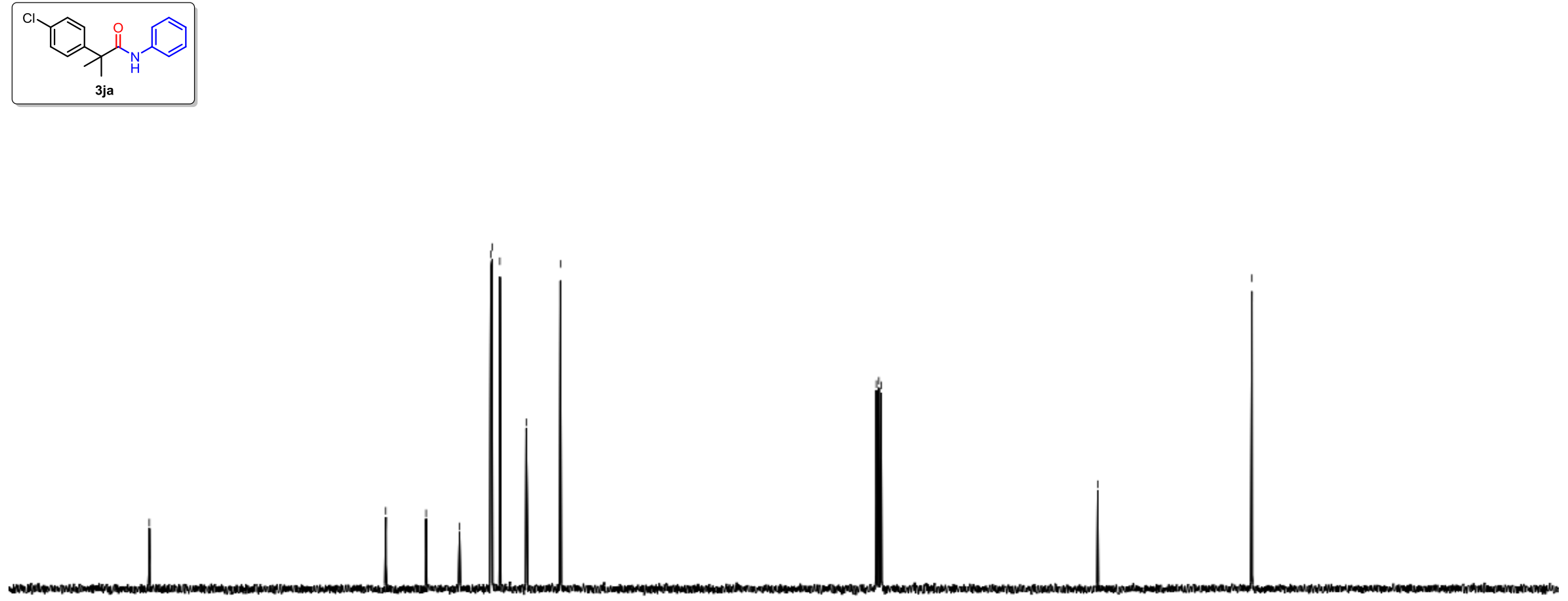

$190 \quad 180$

160

$150 \quad 140$

130

1009

90

80

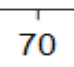

60

50

40

30

20

10

- -10 
${ }^{1} \mathrm{H}$ NMR Spectra (400 MHz, $\mathrm{CDCl}_{3}$ ) of compound 3ka

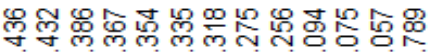

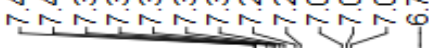
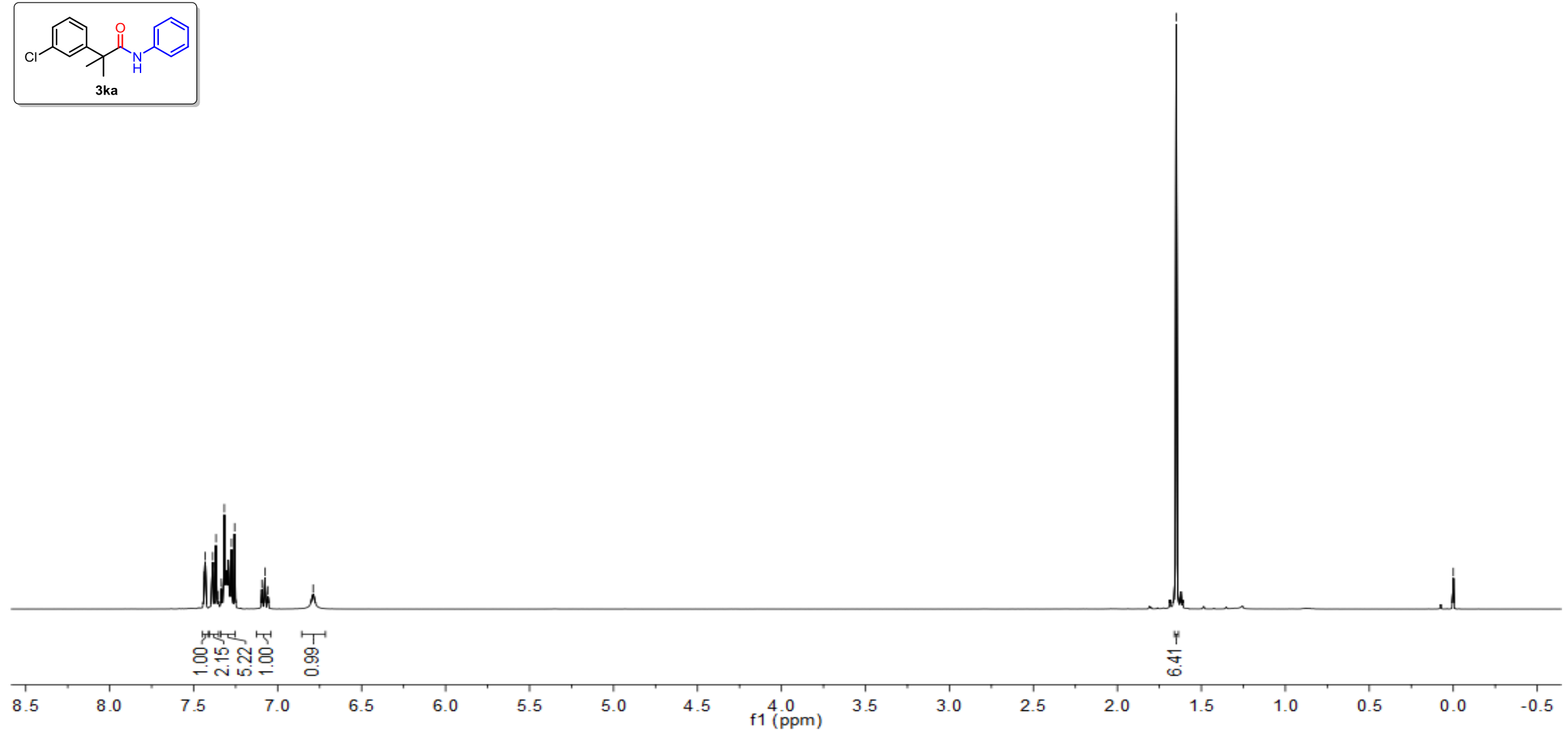
${ }^{13} \mathrm{C}$ NMR Spectra (101 MHz, $\mathrm{CDCl}_{3}$ ) of compound 3ka

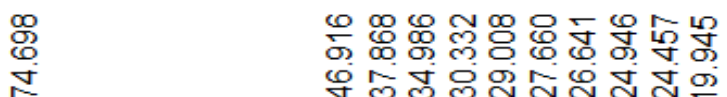

守

$\stackrel{\text { I }}{\stackrel{2}{1}}$

ㅋํำ

숭

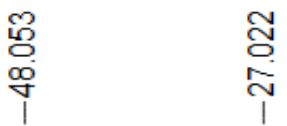
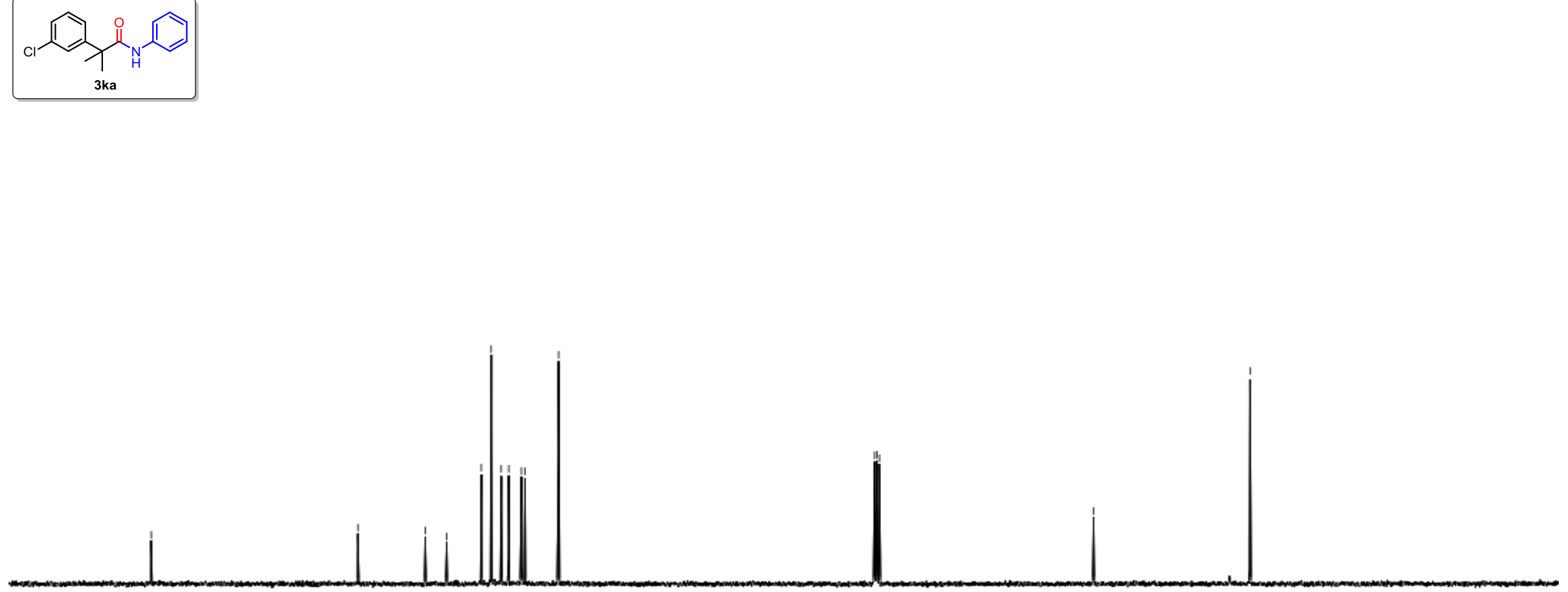

$190 \quad 180$

$\begin{array}{lll}150 & 140 \quad 130\end{array}$

110

10090

90

$80 \quad 70$

60

$50 \quad 40$

$30 \quad 20$

10

$-10$ 
${ }^{1} \mathrm{H}$ NMR Spectra (400 MHz, $\mathrm{CDCl}_{3}$ ) of compound 3la

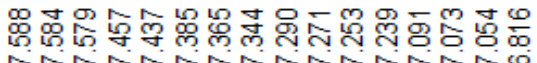

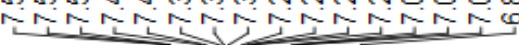
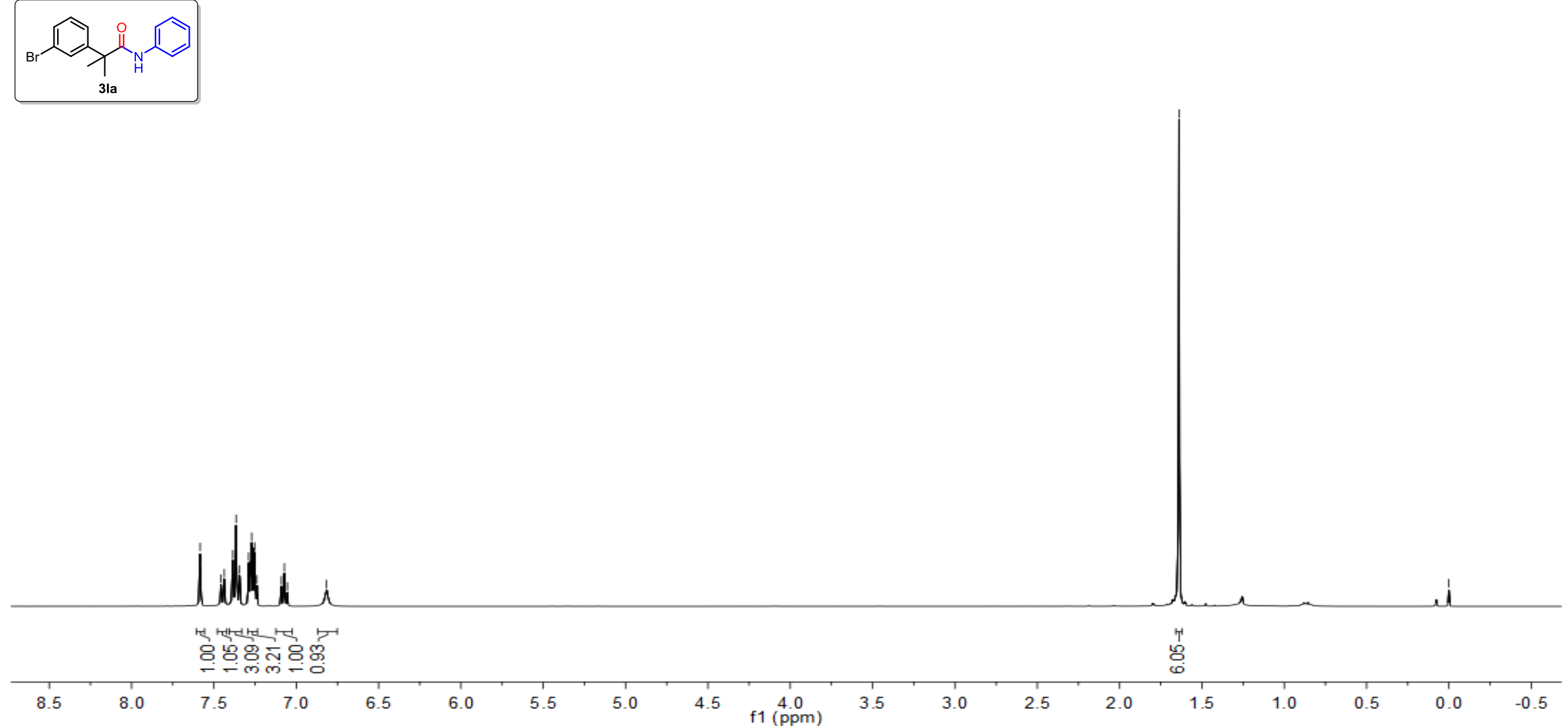
${ }^{13} \mathrm{C}$ NMR Spectra (101 MHz, $\mathrm{CDCl}_{3}$ ) of compound 3la
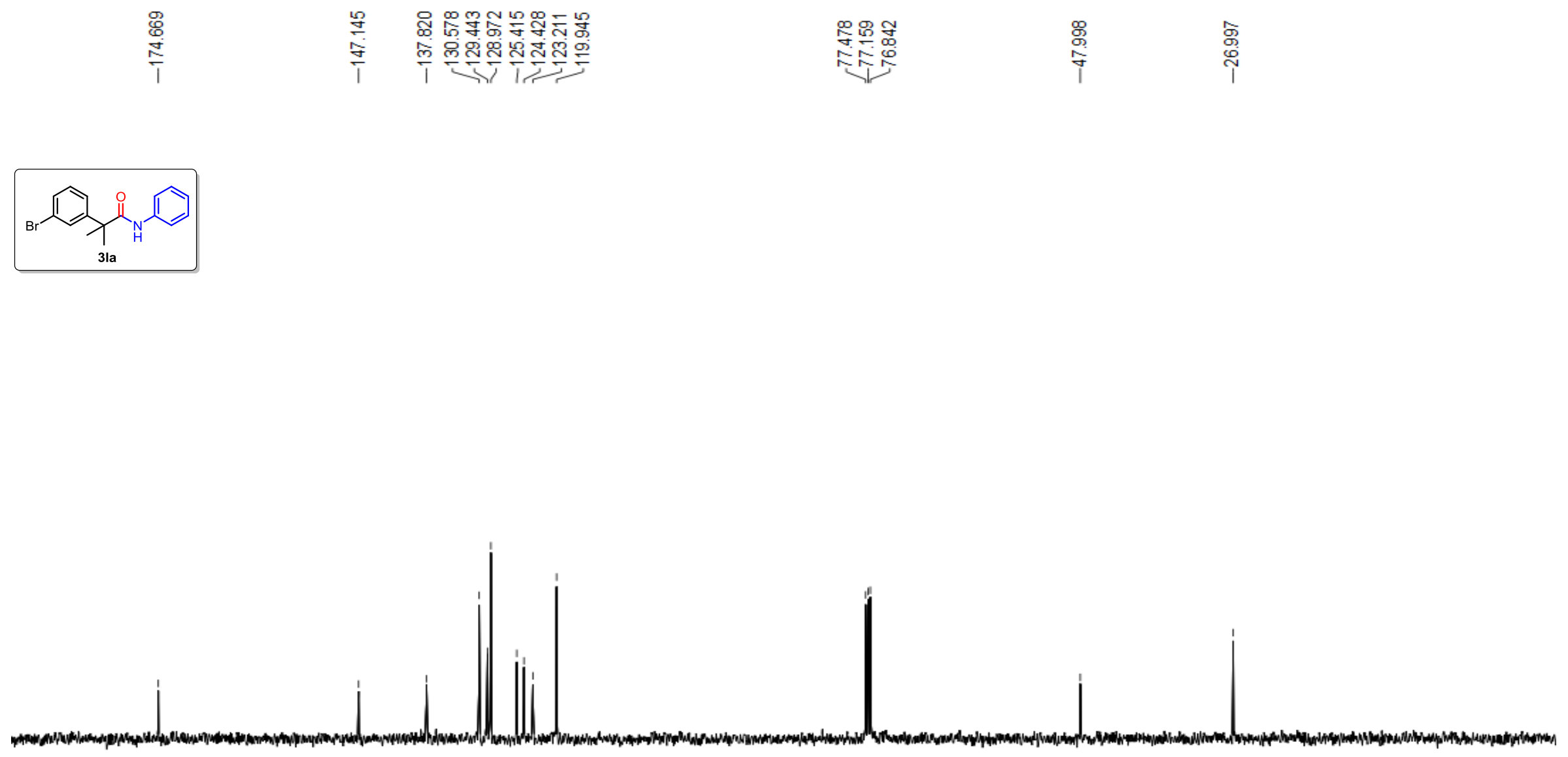
${ }^{1} \mathrm{H}$ NMR Spectra (400 MHz, $\mathrm{CDCl}_{3}$ ) of compound 3ma

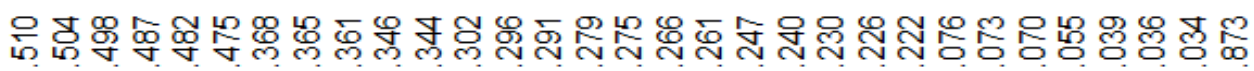

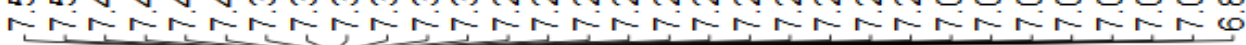
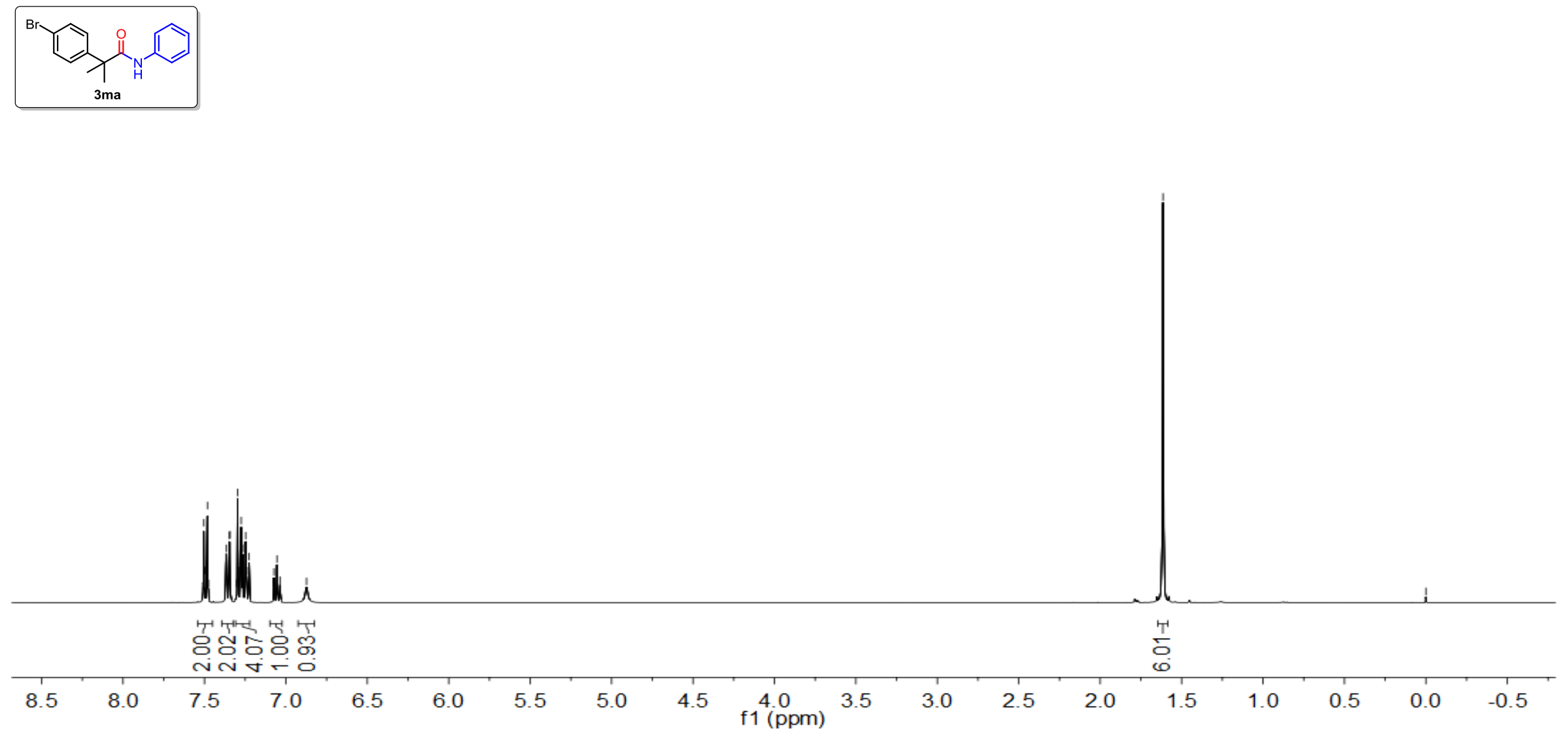
${ }^{13} \mathrm{C}$ NMR Spectra (101 MHz, $\mathrm{CDCl}_{3}$ ) of compound 3ma
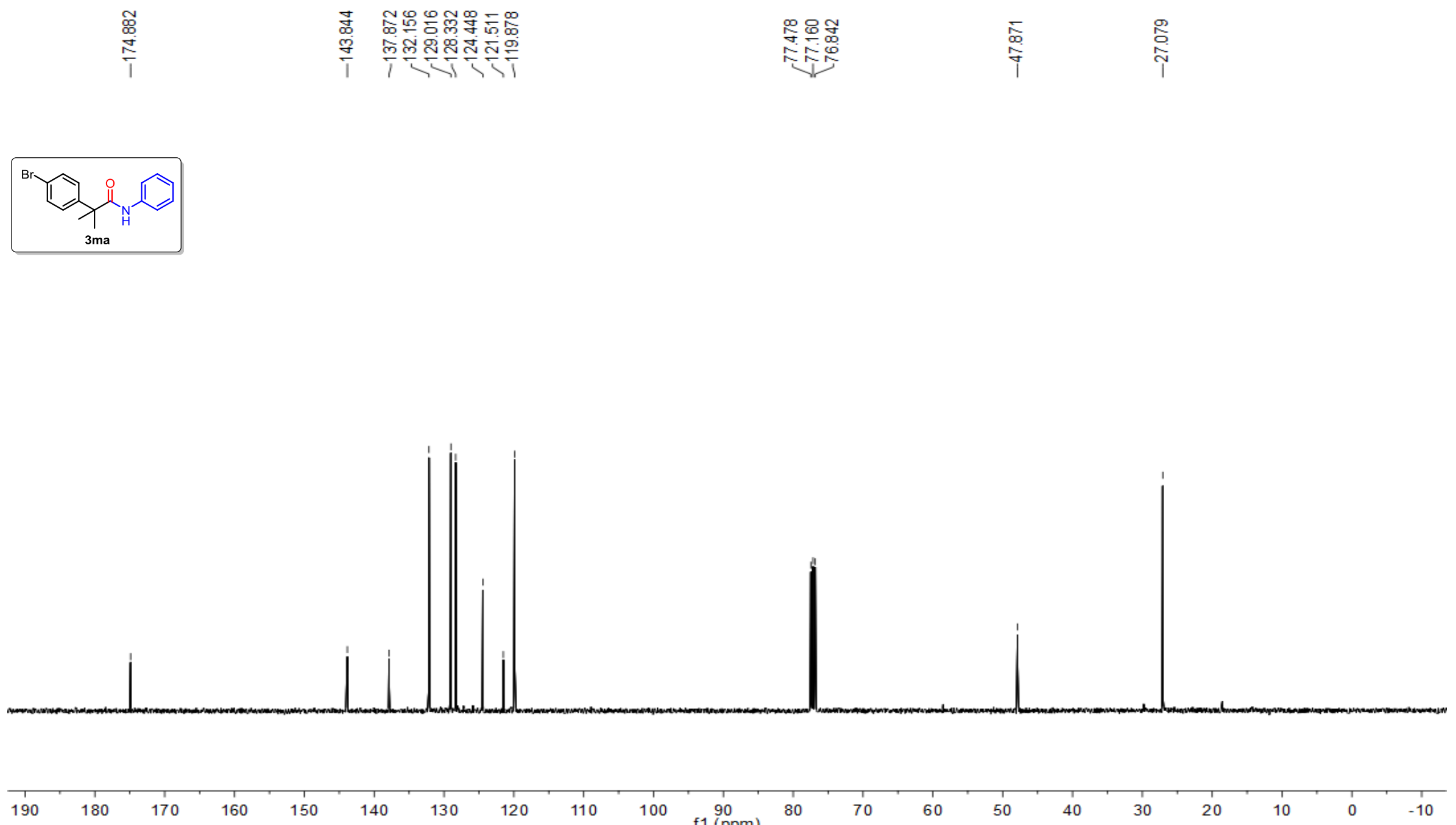

110

100

f1 900

S116


${ }^{1} \mathrm{H}$ NMR Spectra (400 MHz, $\mathrm{CDCl}_{3}$ ) of compound 3na

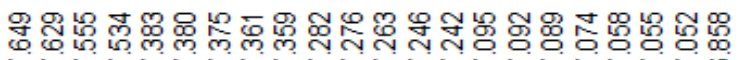

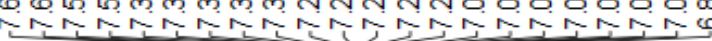
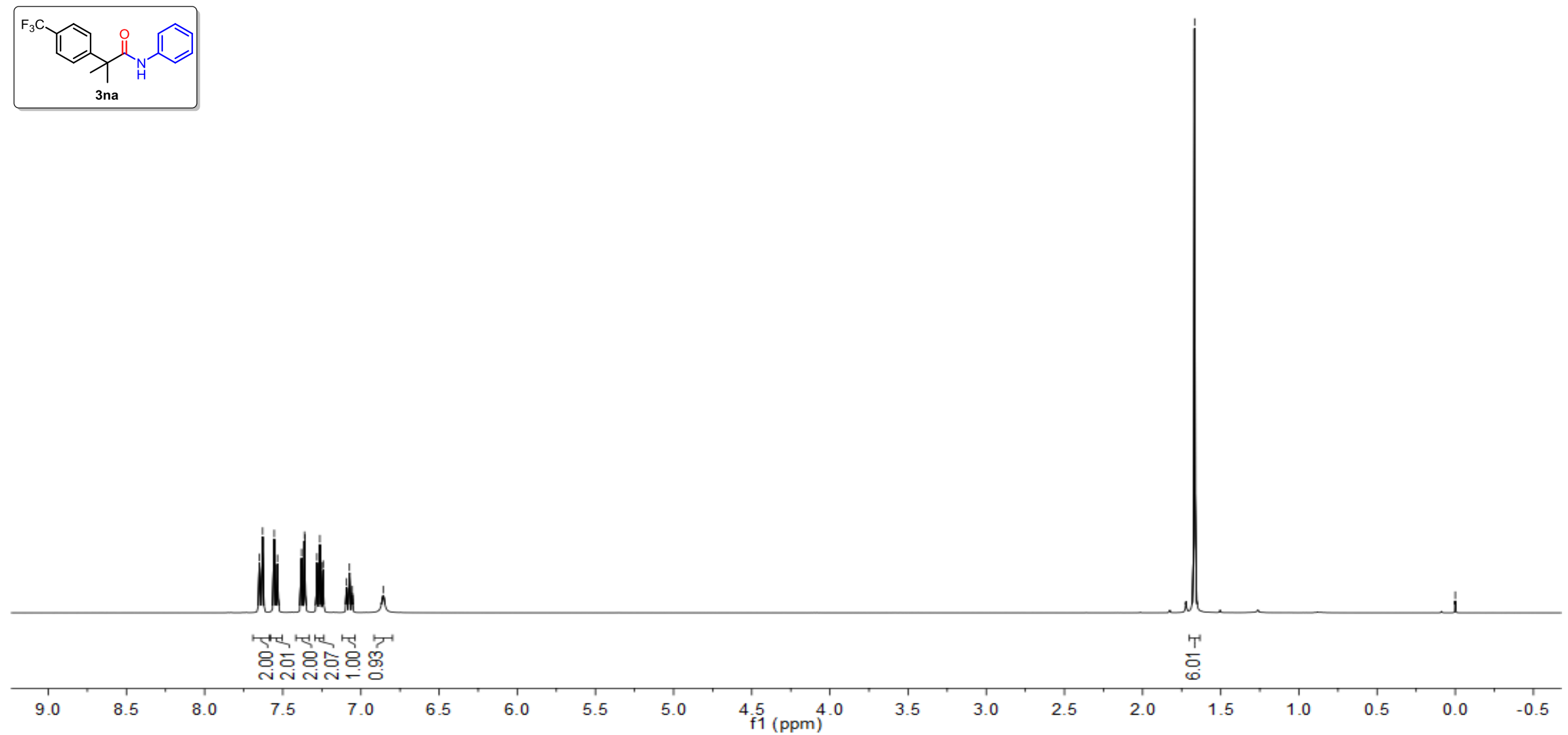
${ }^{13} \mathrm{C}$ NMR Spectra $\left(101 \mathrm{MHz}, \mathrm{CDCl}_{3}\right)$ of compound 3na

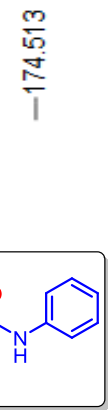

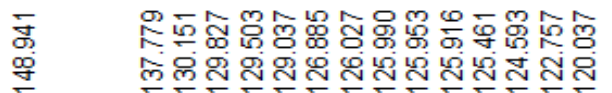

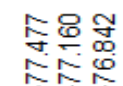

$\underset{\substack{0 \\ \text { ơ }}}{\stackrel{9}{0}}$
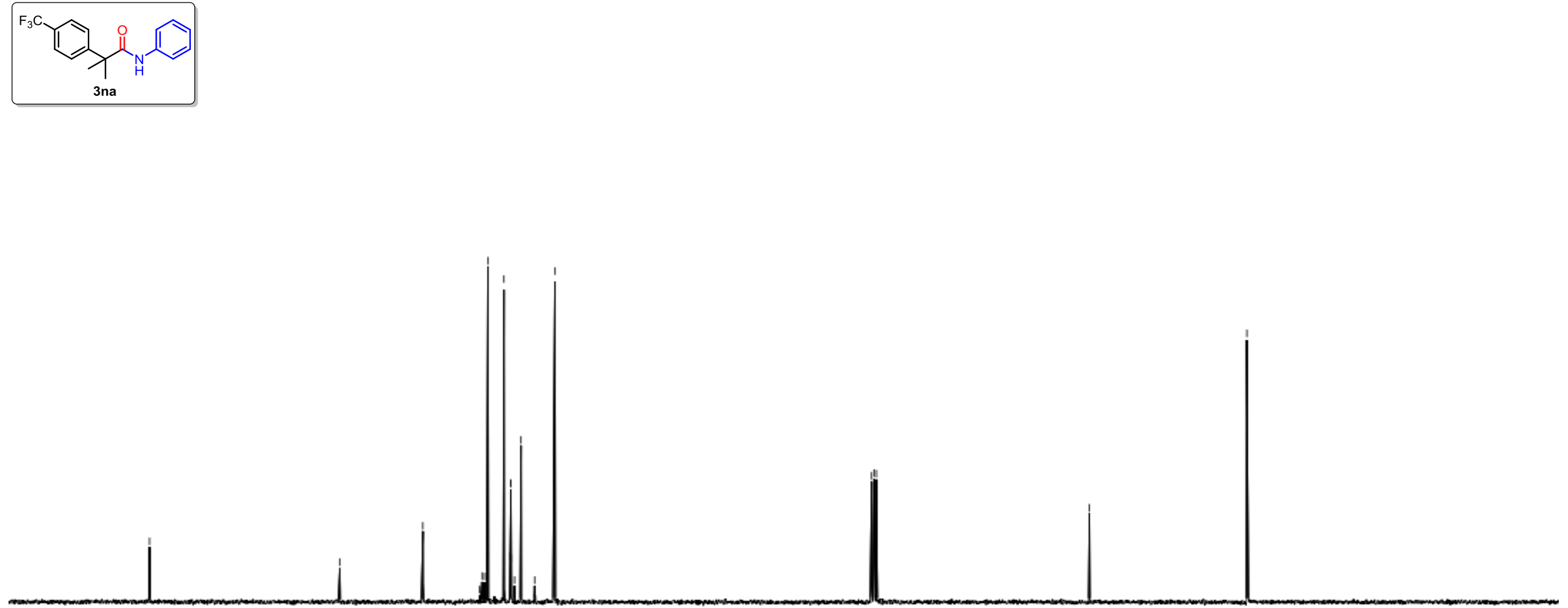
${ }^{19} \mathrm{~F}$ NMR Spectra (375 MHz, $\mathrm{CDCl}_{3}$ ) of compound 3na

$$
\underset{\substack{i \\ i}}{\stackrel{\infty}{7}}
$$
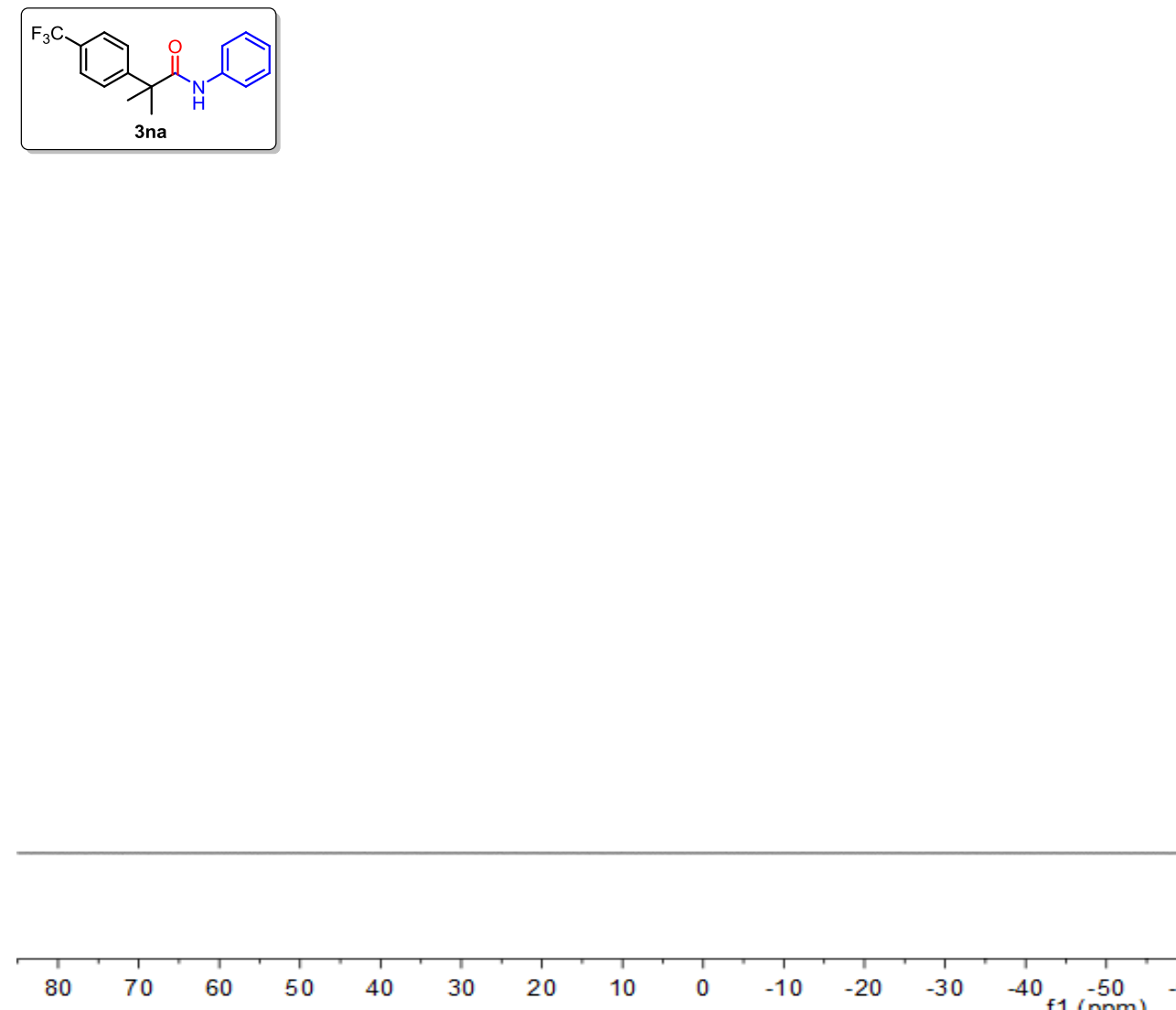

S119 
${ }^{1} \mathrm{H}$ NMR Spectra (400 MHz, $\mathrm{CDCl}_{3}$ ) of compound 3oa

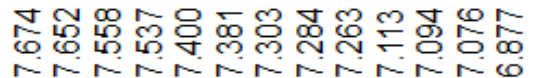

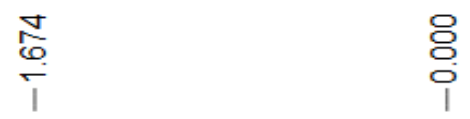
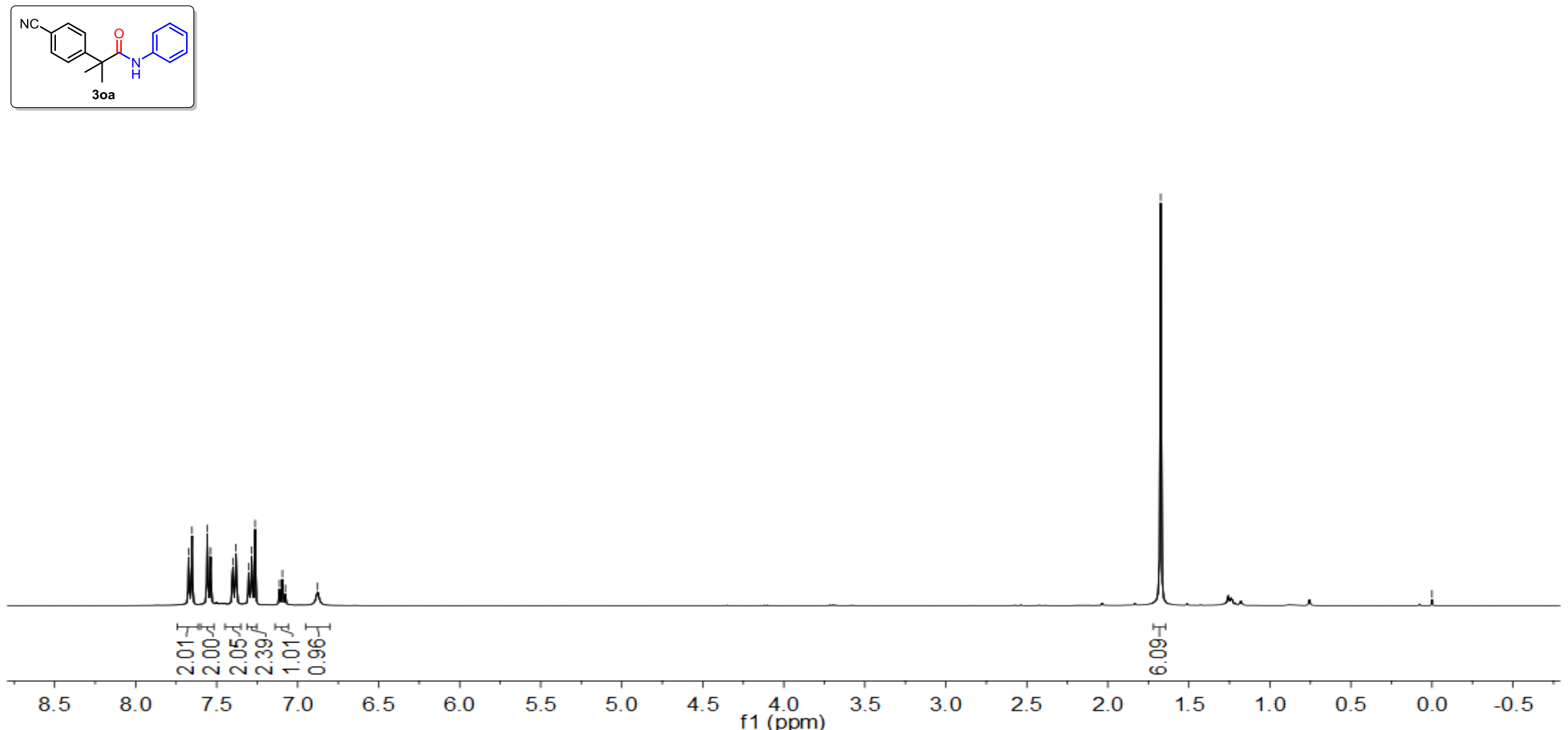
${ }^{13} \mathrm{C}$ NMR Spectra (101 MHz, $\mathrm{CDCl}_{3}$ ) of compound 3oa
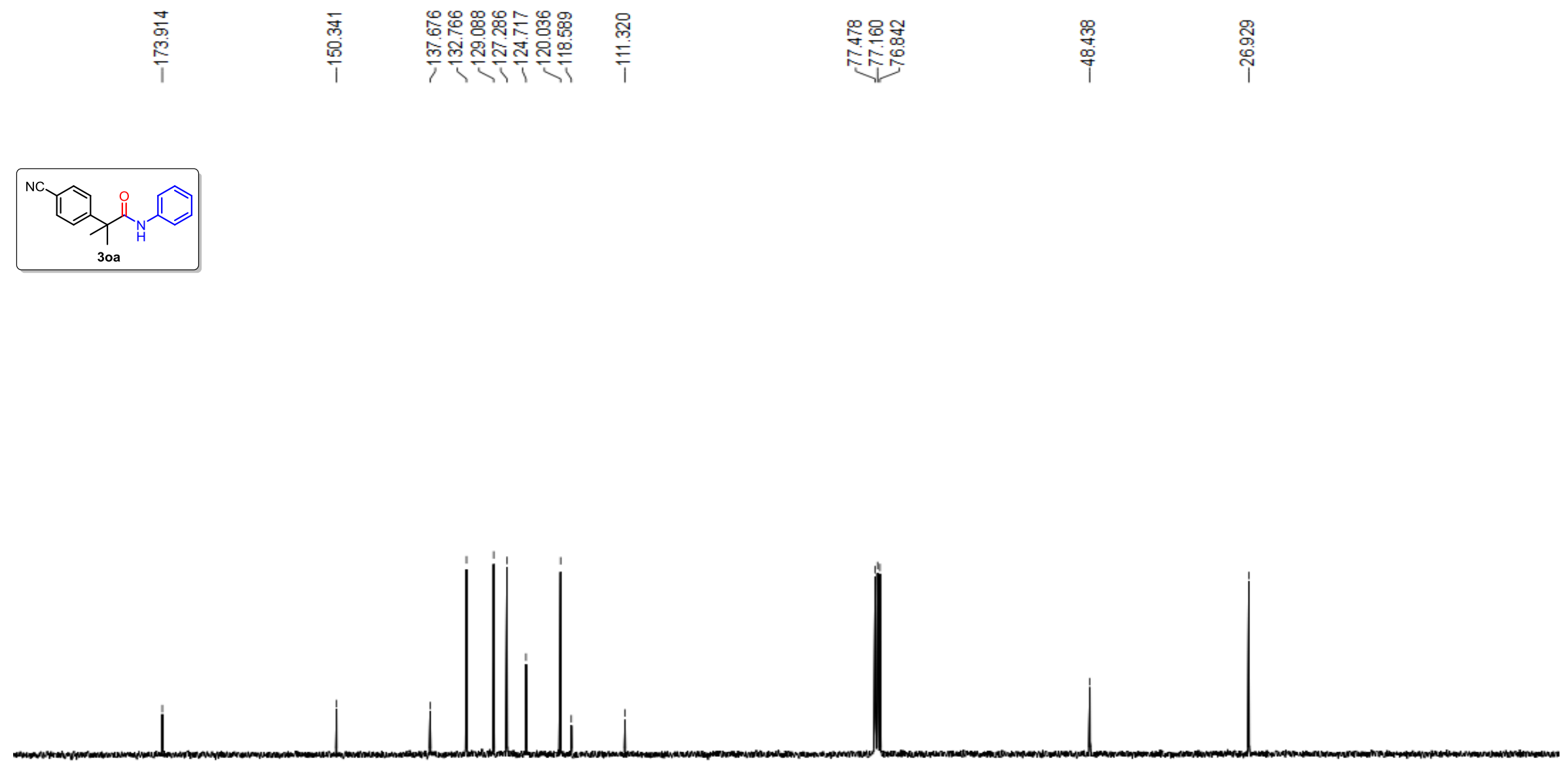
${ }^{1} \mathrm{H}$ NMR Spectra (400 MHz, $d^{6}$-DMSO) of compound 3pa

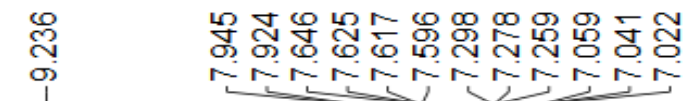

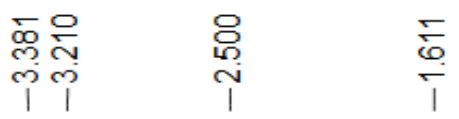
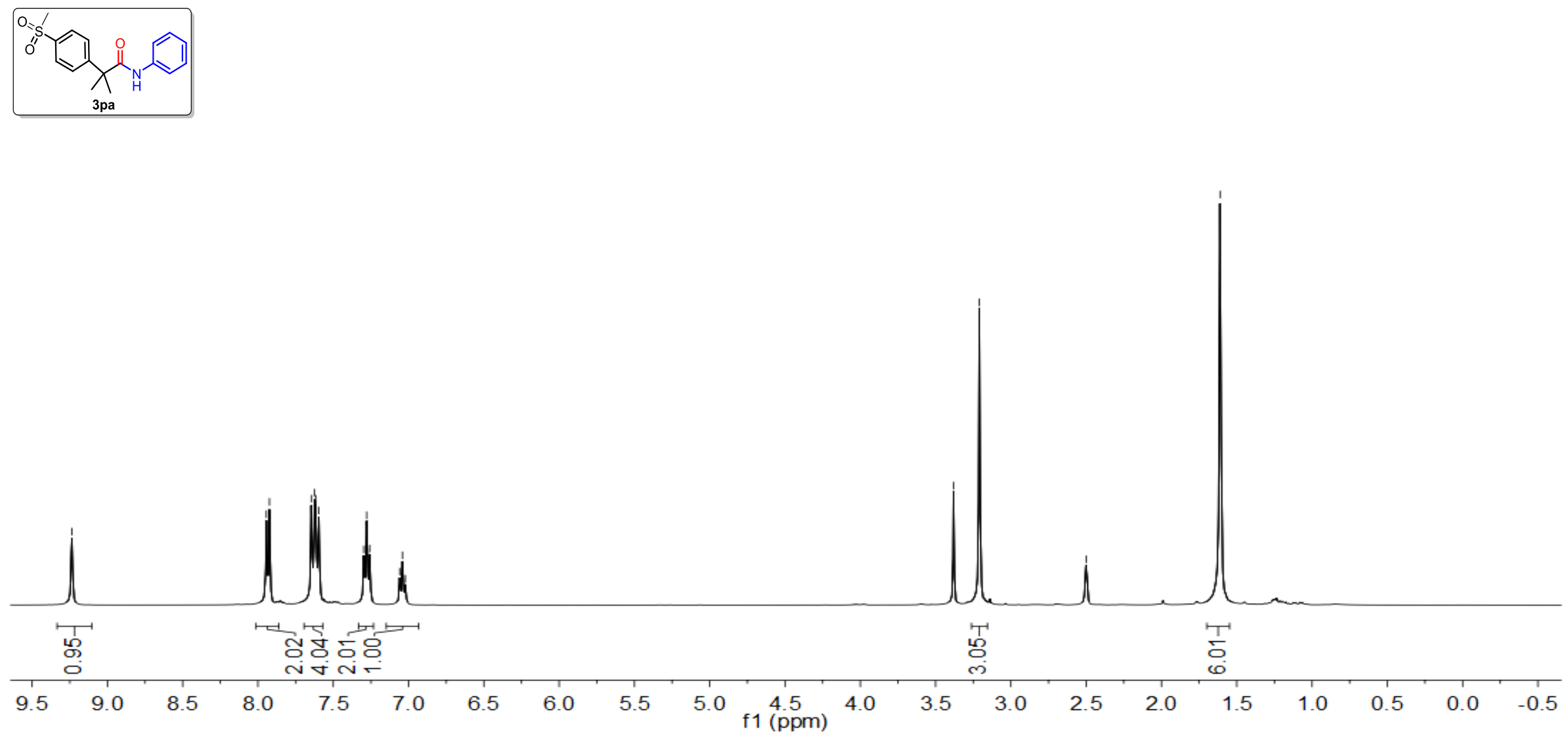
${ }^{13} \mathrm{C}$ NMR Spectra (101 MHz, C $d^{6}$-DMSO) of compound 3pa

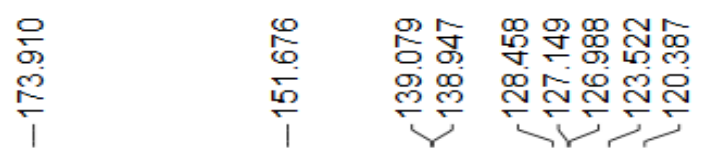

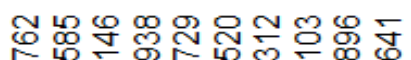

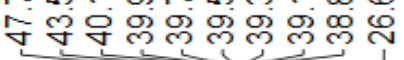
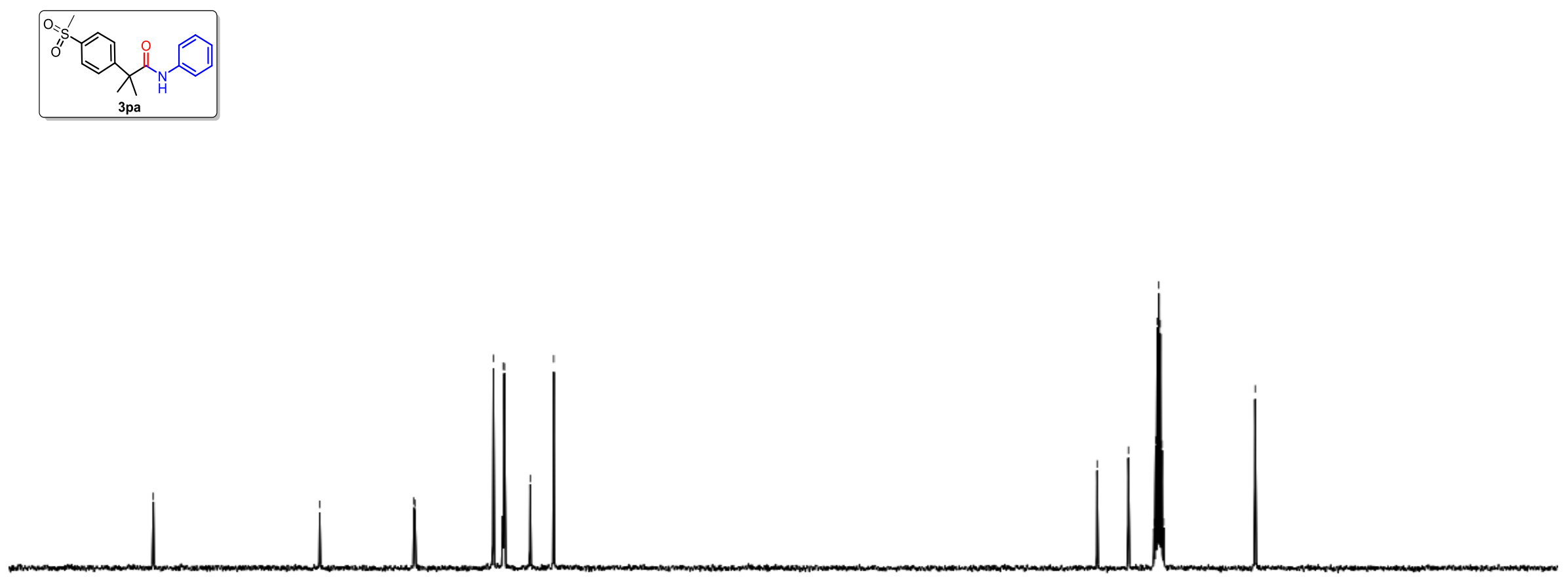

$190 \quad 180$

$170 \quad 160$

$150 \quad 140$

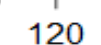

110

1009

90

80

70

60

50

40

30

20

10

$-10$ 
${ }^{1} \mathrm{H}$ NMR Spectra (400 MHz, $\mathrm{CDCl}_{3}$ ) of compound 3qa

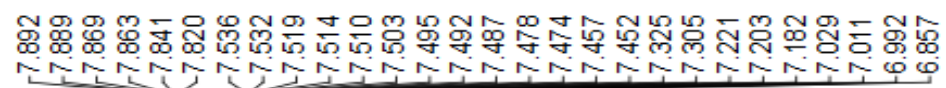
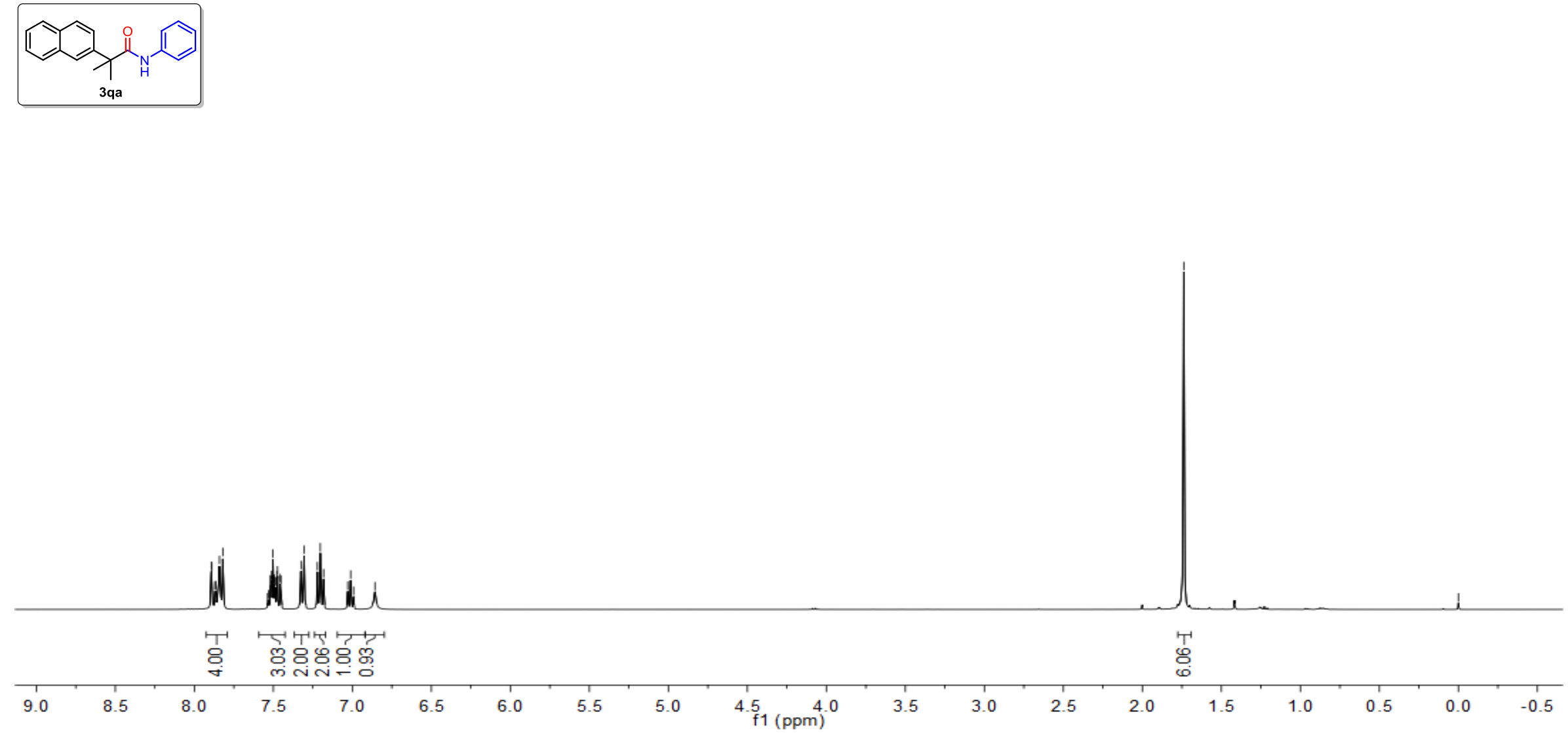
${ }^{13} \mathrm{C}$ NMR Spectra (101 MHz, $\mathrm{CDCl}_{3}$ ) of compound $3 \mathbf{q a}$

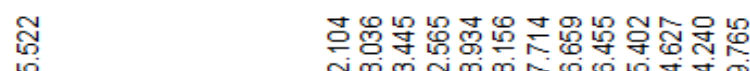

每
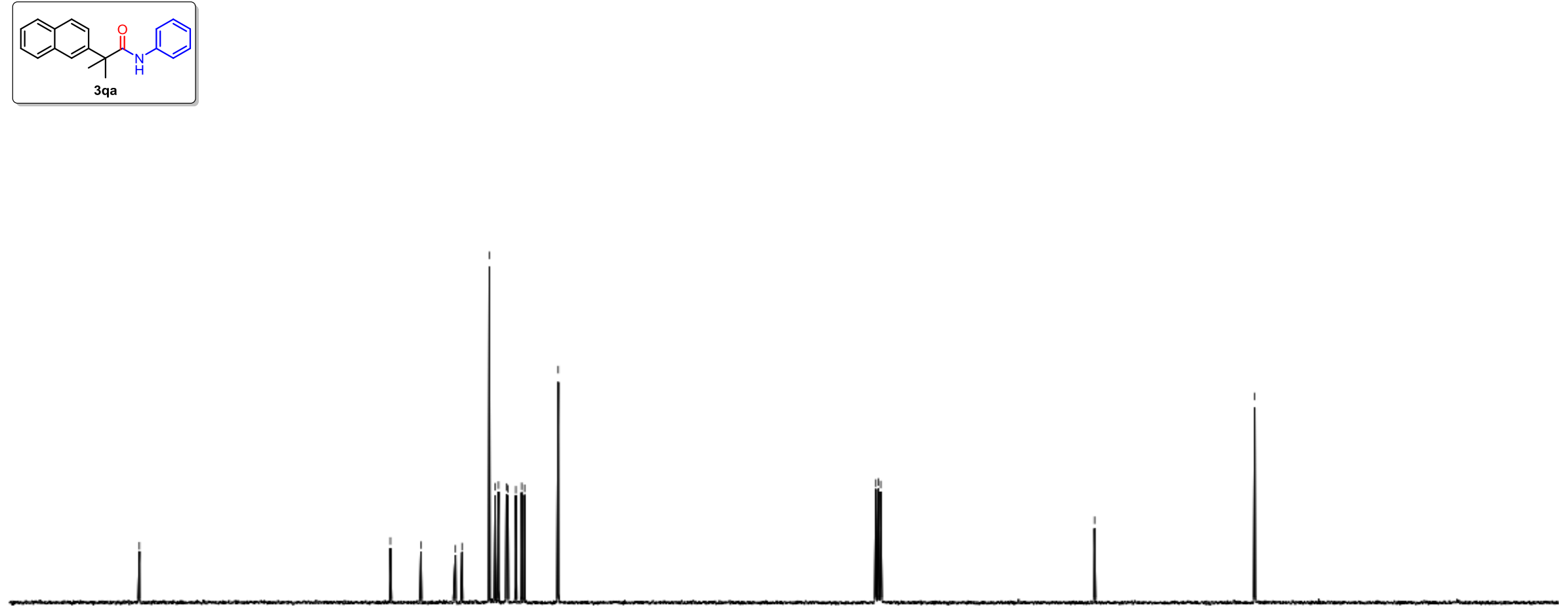

190180

$160 \quad 150$

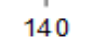

130

120

110

100

$\stackrel{90}{90}$

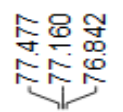

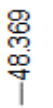

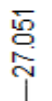

广 
${ }^{1} \mathrm{H}$ NMR Spectra (400 MHz, $\mathrm{CDCl}_{3}$ ) of compound 3ra

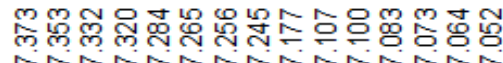

Nincising
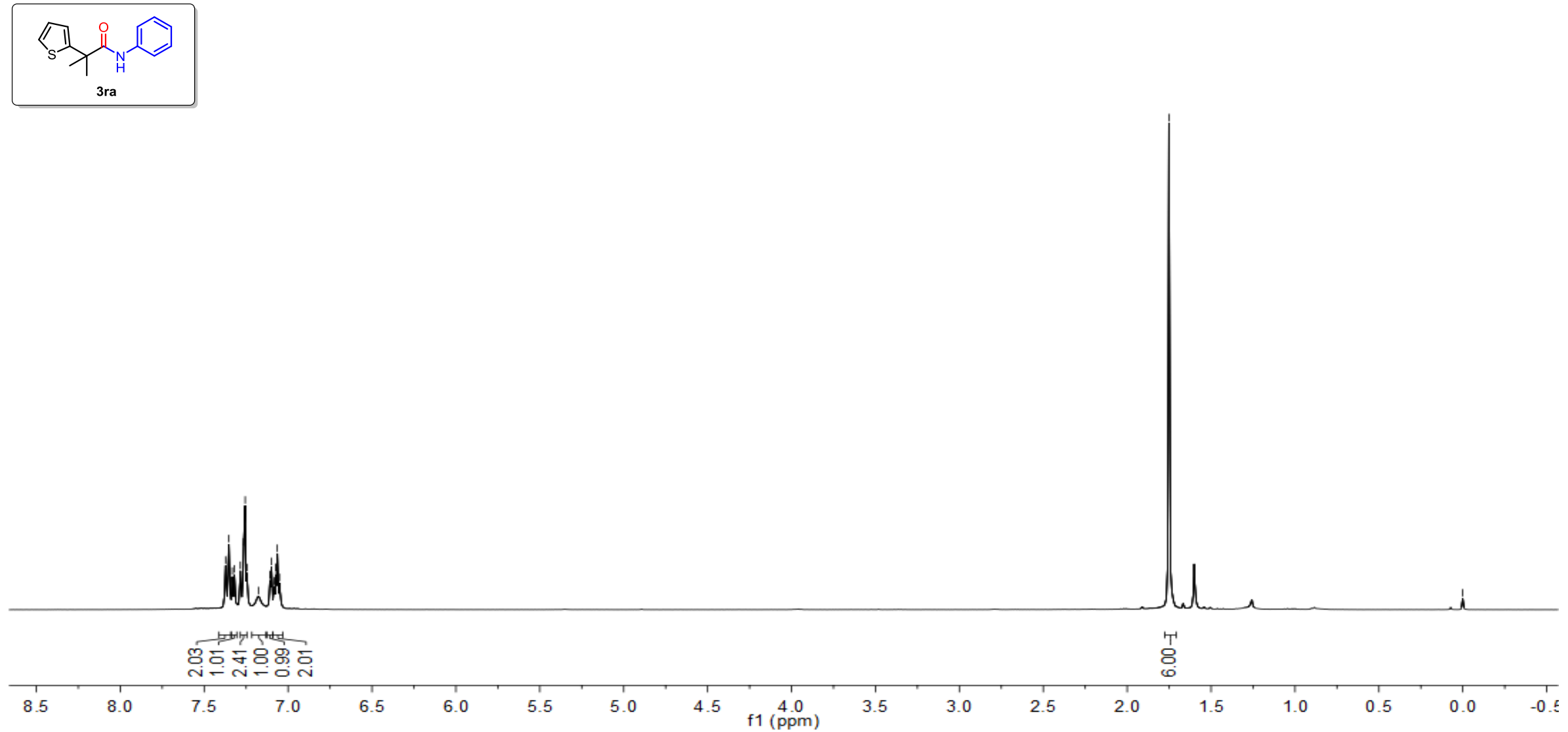
${ }^{13} \mathrm{C}$ NMR Spectra (101 MHz, $\mathrm{CDCl}_{3}$ ) of compound 3ra
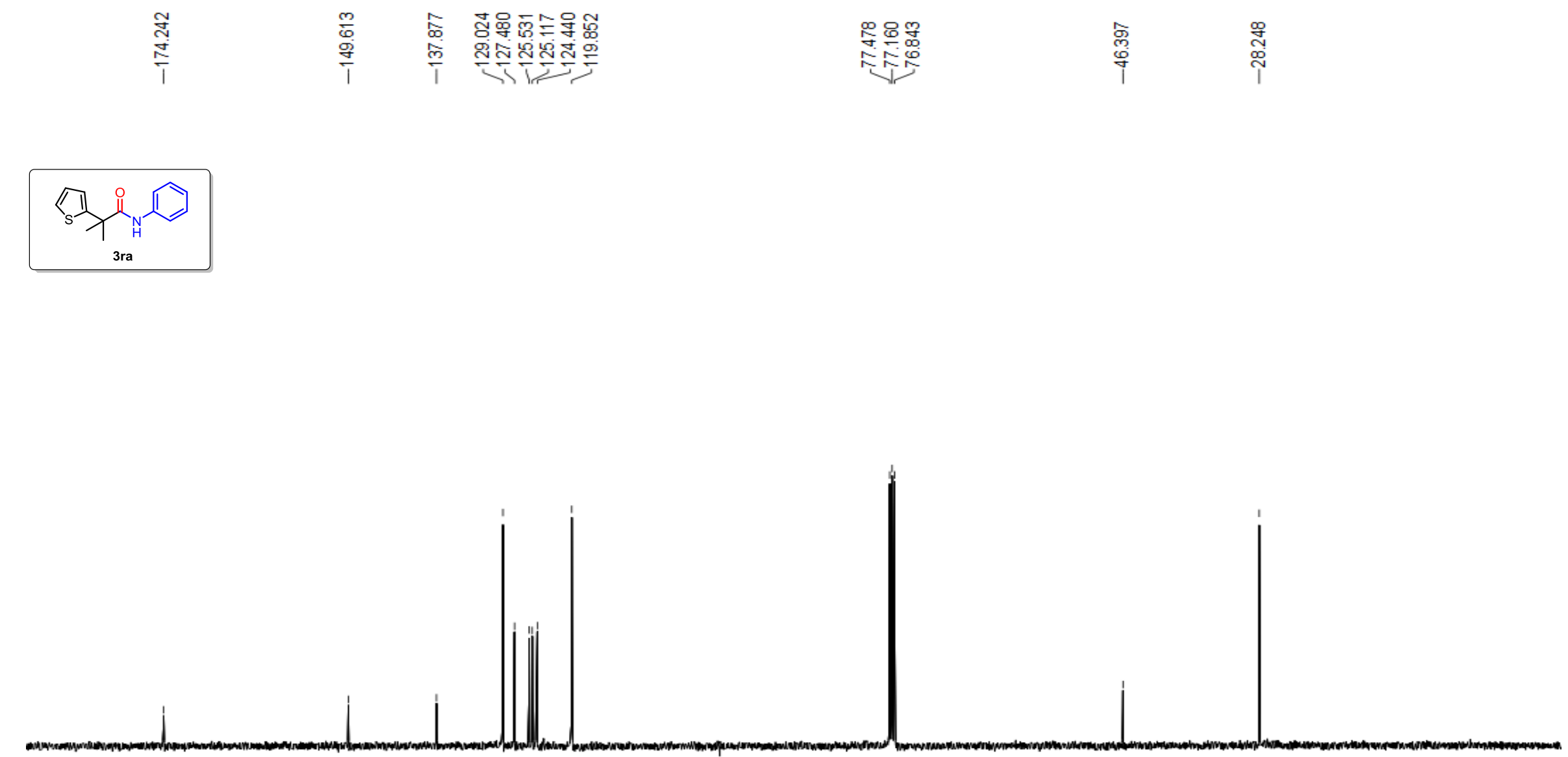

190

180

170

$160 \quad 150$

$140 \quad 130$

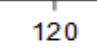

$100 \quad 90 \quad 80$
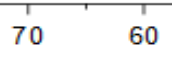

50

40

10 
${ }^{1} \mathrm{H}$ NMR Spectra $\left(600 \mathrm{MHz}, \mathrm{CDCl}_{3}\right.$ ) of compound 3sa

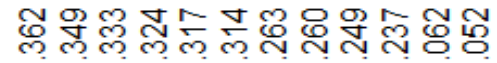

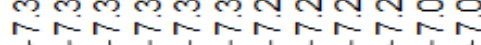

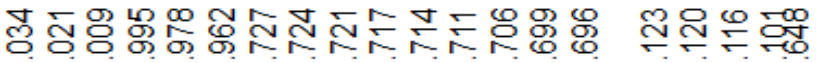

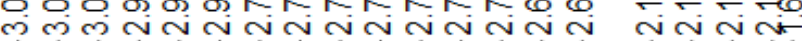
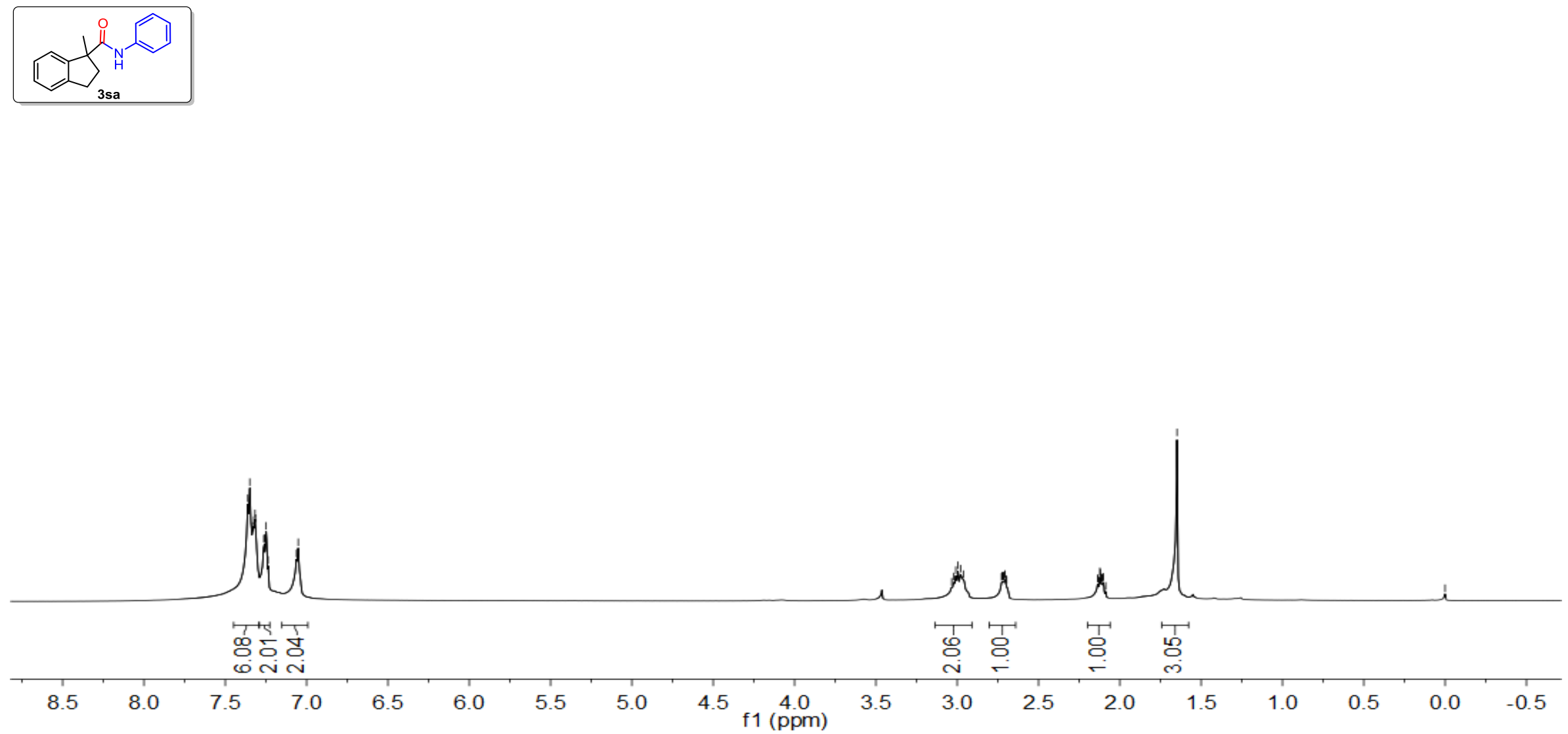
${ }^{13} \mathrm{C}$ NMR Spectra (151 MHz, $\mathrm{CDCl}_{3}$ ) of compound 3sa
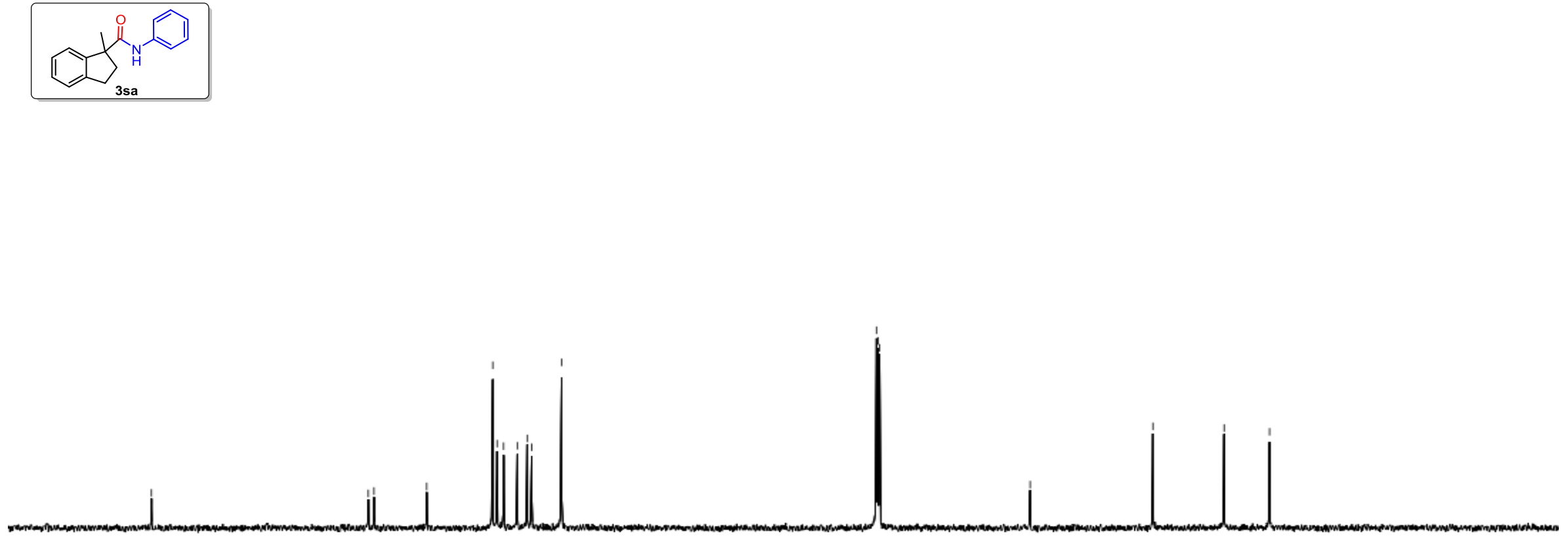

$190 \quad 180 \quad 170$ 
${ }^{1} \mathrm{H}$ NMR Spectra (400 MHz, $\mathrm{CDCl}_{3}$ ) of compound 3ta

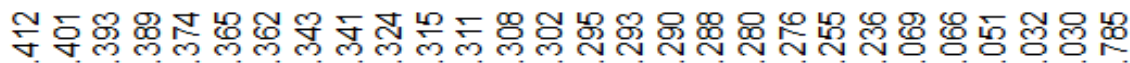

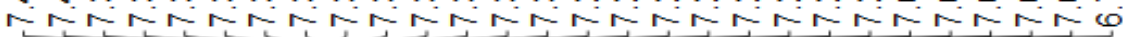

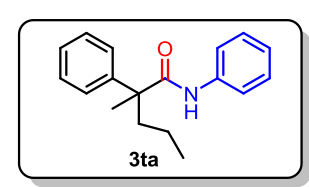

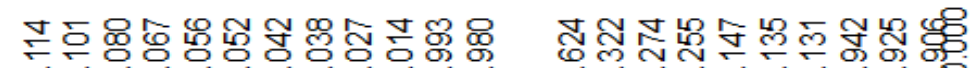

NunnNminj;

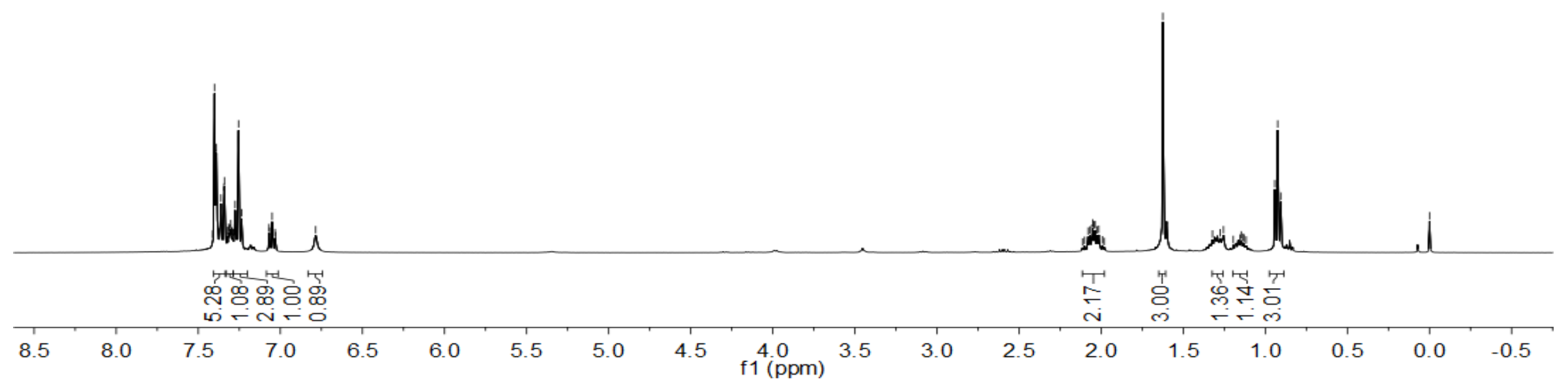


${ }^{13} \mathrm{C}$ NMR Spectra (101 MHz, $\mathrm{CDCl}_{3}$ ) of compound 3ta

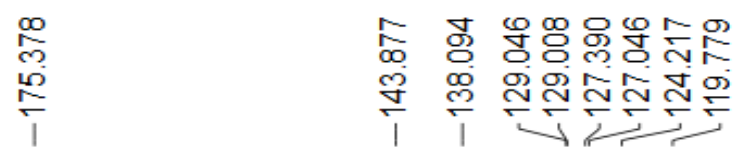

F⿻응
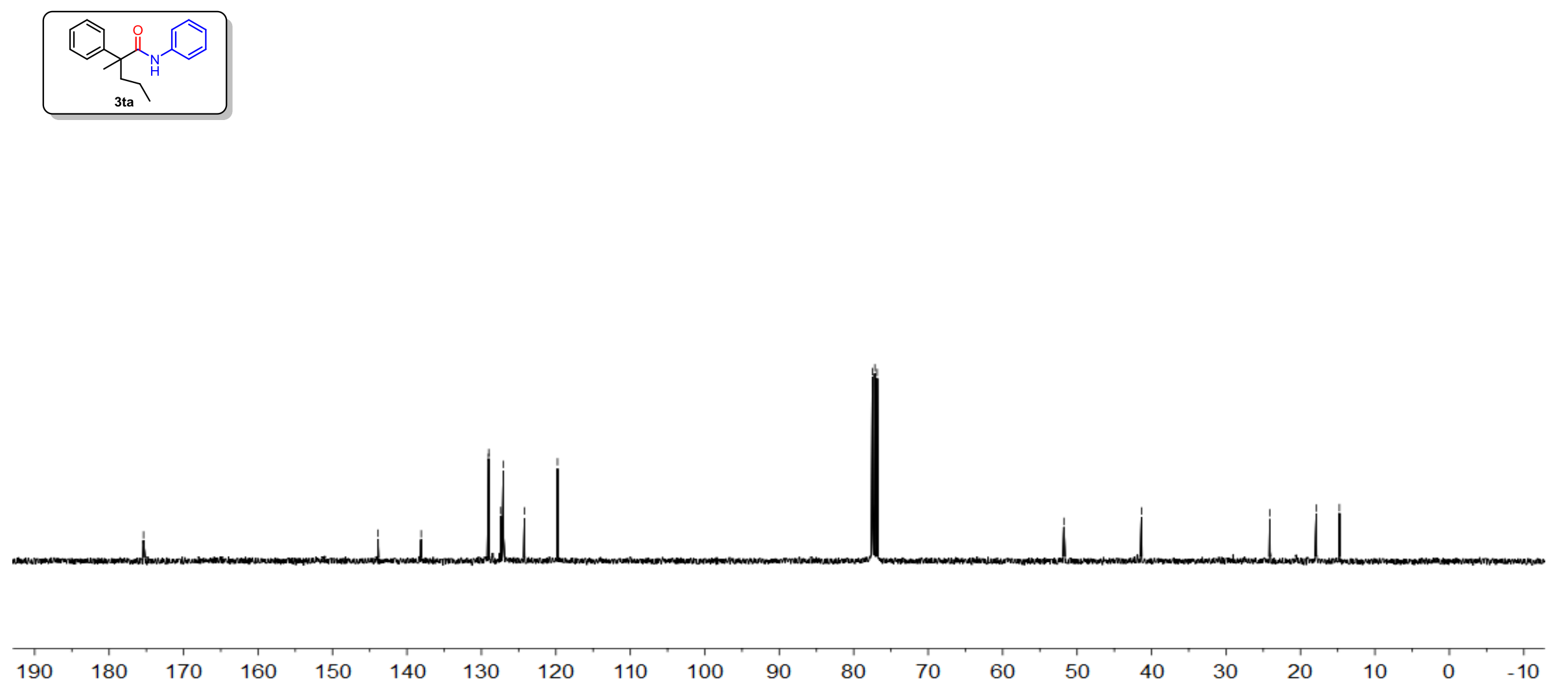
${ }^{1} \mathrm{H}$ NMR Spectra (400 MHz, $\mathrm{CDCl}_{3}$ ) of compound 3ua

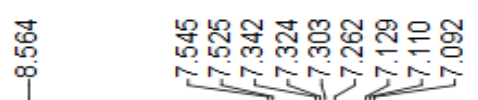

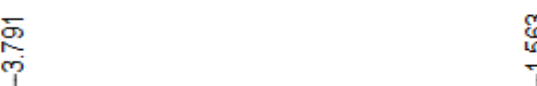

@:
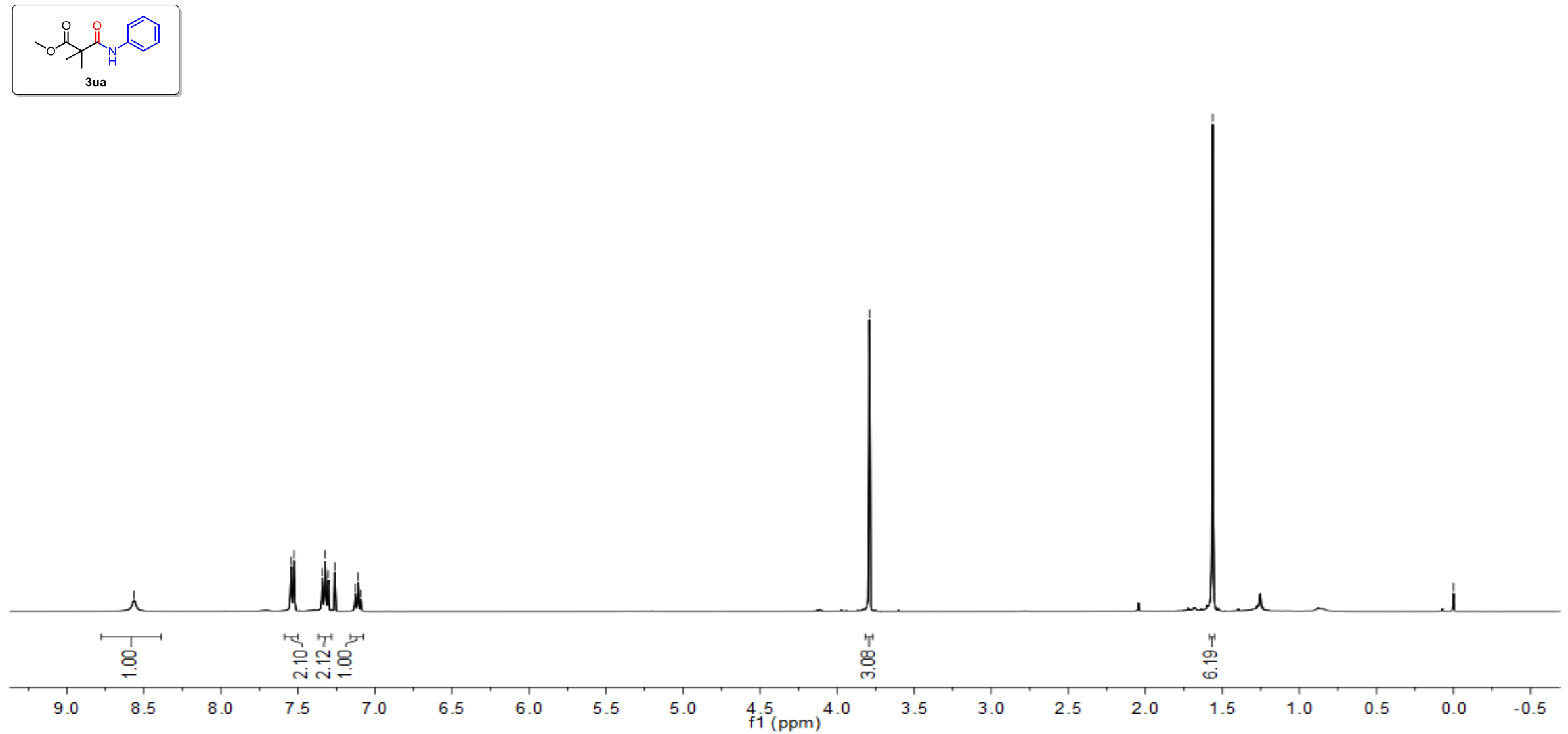
${ }^{13} \mathrm{C}$ NMR Spectra (101 MHz, $\mathrm{CDCl}_{3}$ ) of compound 3ua

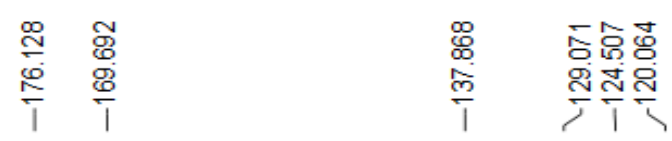

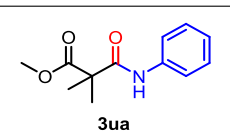

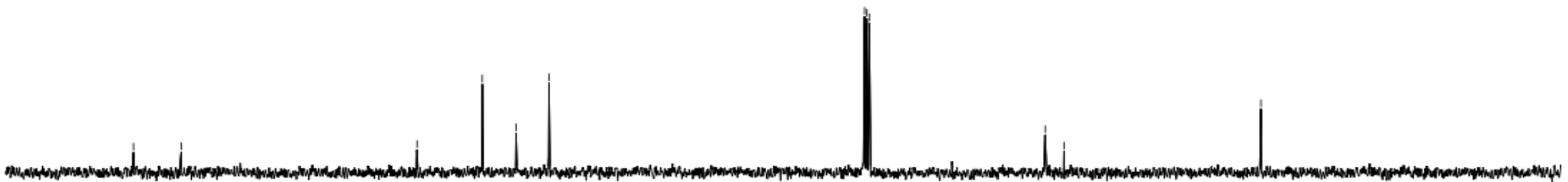


${ }^{1} \mathrm{H}$ NMR Spectra $\left(600 \mathrm{MHz}, \mathrm{CDCl}_{3}\right)$ of compound 3ve

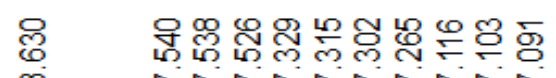

$1 \quad \underbrace{\infty}$

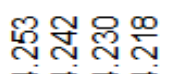

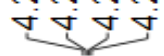

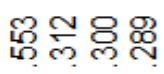

正

응
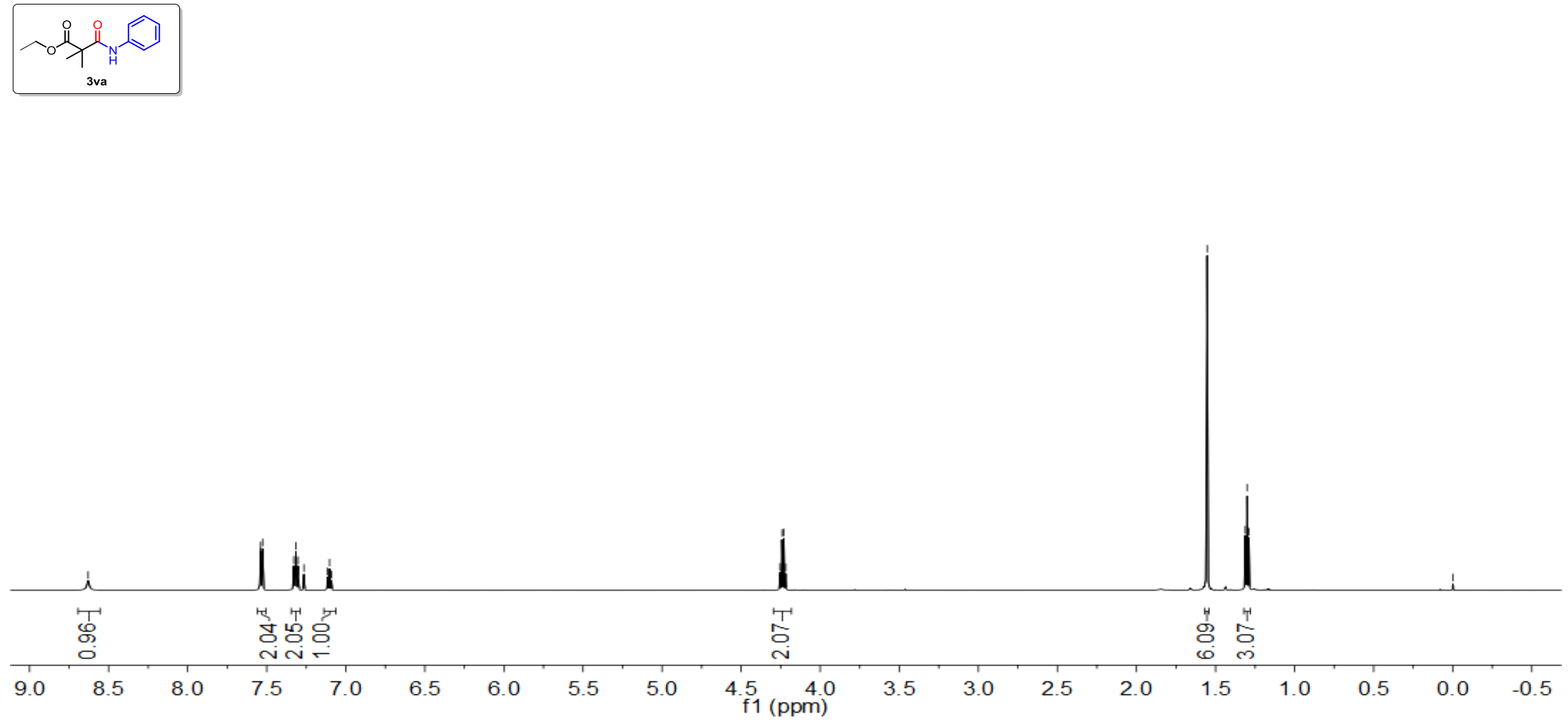

S134 
${ }^{13} \mathrm{C}$ NMR Spectra (151 MHz, $\mathrm{CDCl}_{3}$ ) of compound 3va

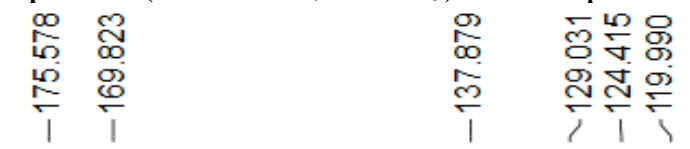

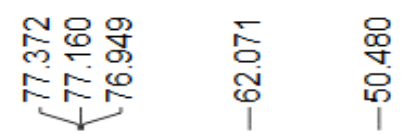

$\underset{\substack{\text { D } \\ \text { i }}}{\stackrel{\text { g }}{+}}$
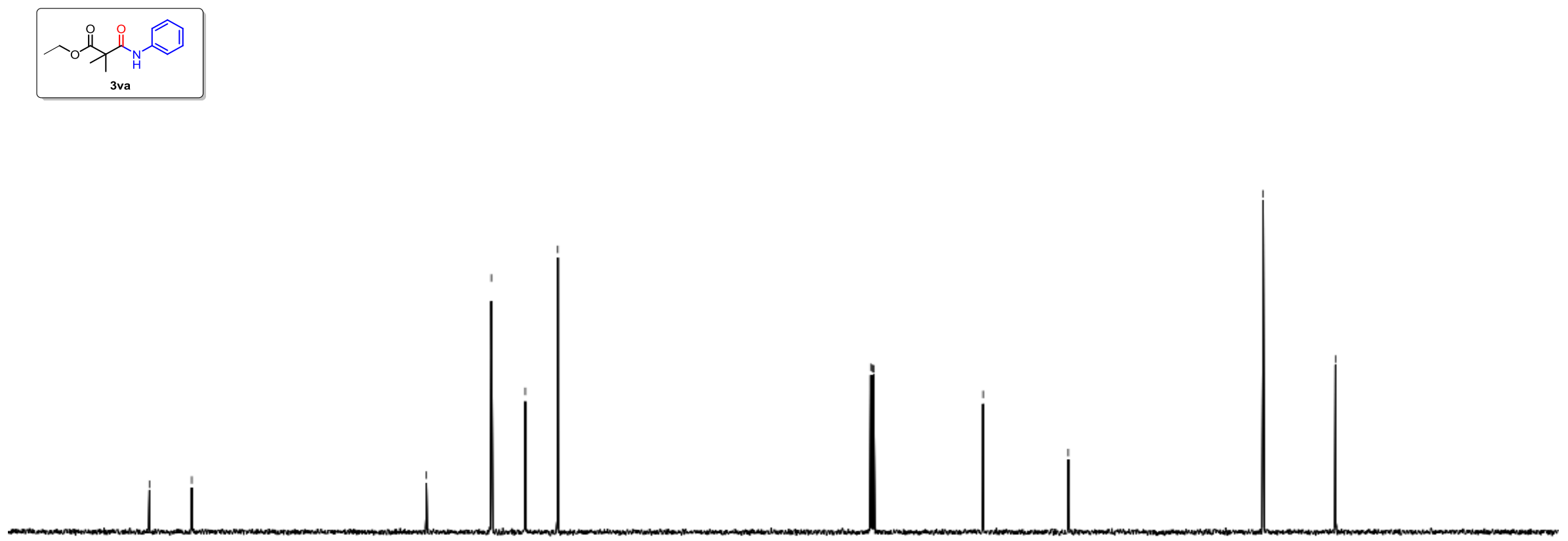

190

$180 \quad 170 \quad 160$

150

$140 \quad 130 \quad 120$

$110 \quad 100 \underset{\mathrm{f} 1}{\stackrel{90}{(\mathrm{ppm})}} 80$

$70 \quad 60$

$50 \quad 40 \quad 30$

20

S135 
${ }^{1} \mathrm{H}$ NMR Spectra (400 MHz, $\mathrm{CDCl}_{3}$ ) of compound 3wa

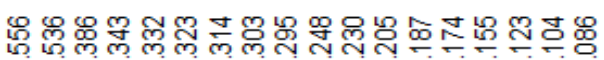

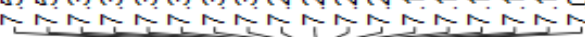
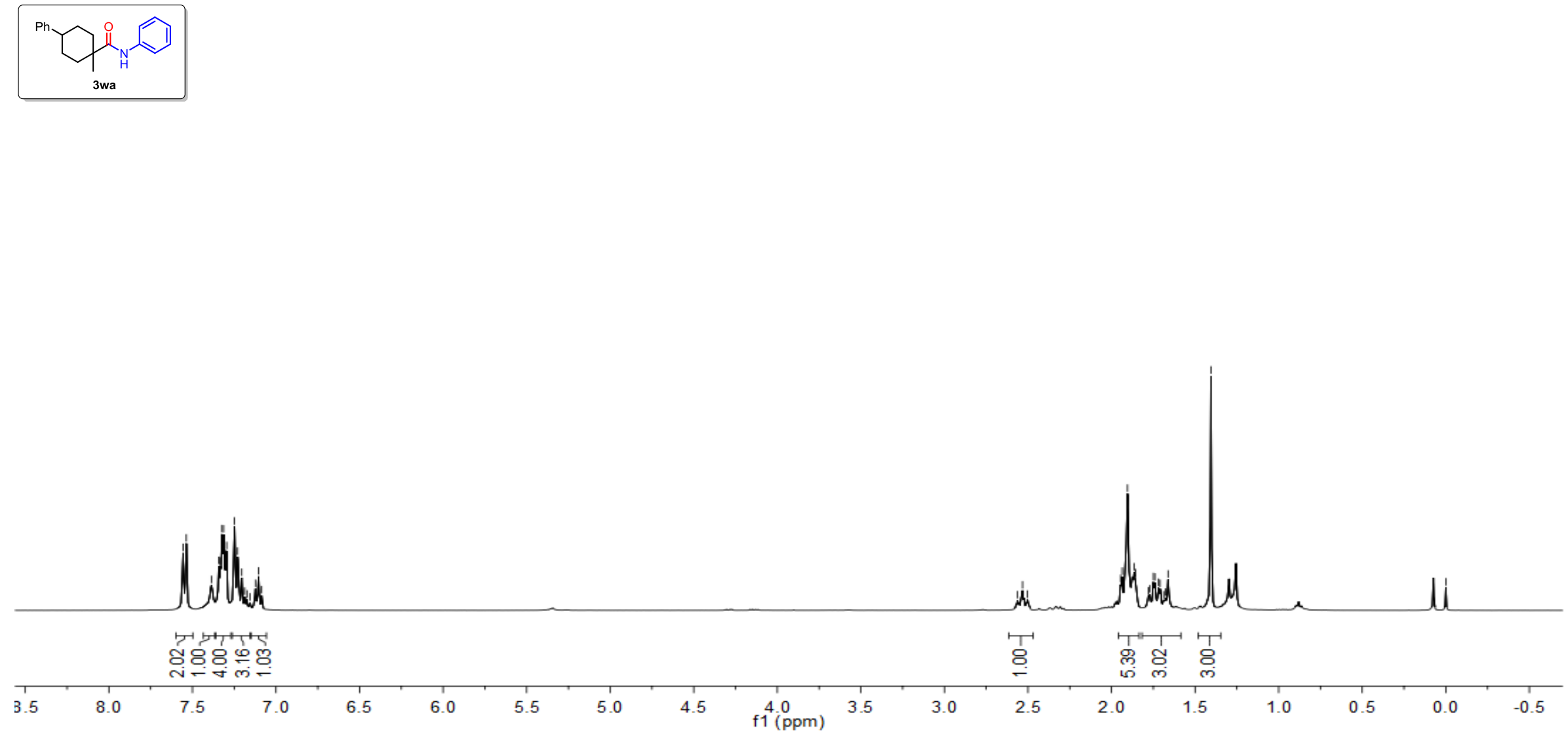
${ }^{13} \mathrm{C}$ NMR Spectra $\left(101 \mathrm{MHz}, \mathrm{CDCl}_{3}\right.$ ) of compound 3wa
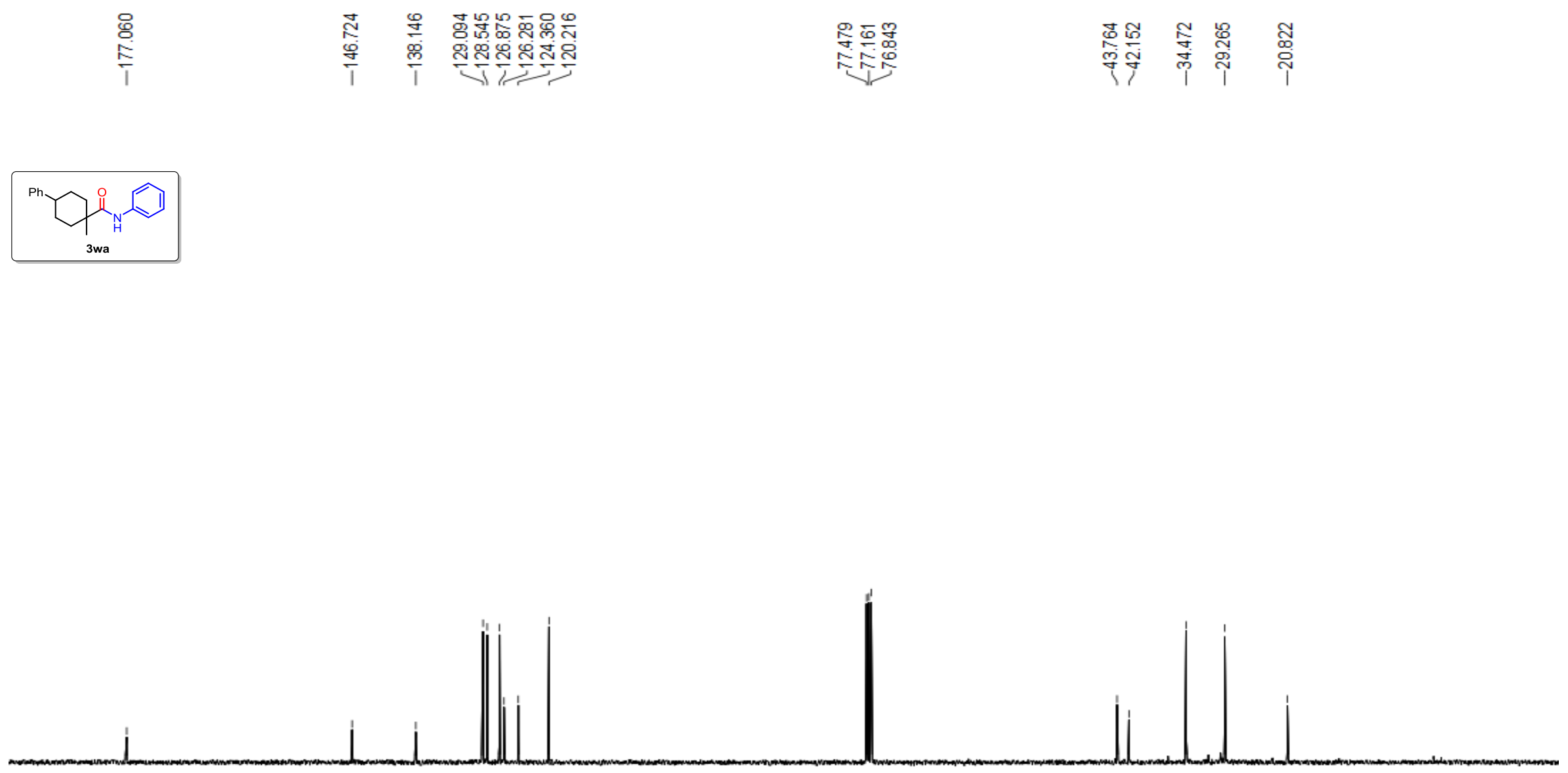

$190 \quad 18$

170

$160 \quad 150$

140

120

$110 \quad 100$

$90 \quad 80$

70

$60 \quad 50$

40

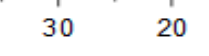


${ }^{1} \mathrm{H}$ NMR Spectra (400 MHz, $\mathrm{CDCl}_{3}$ ) of compound 3xa

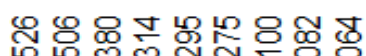

ririnis

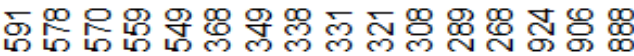

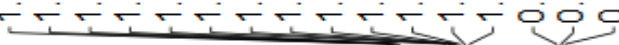
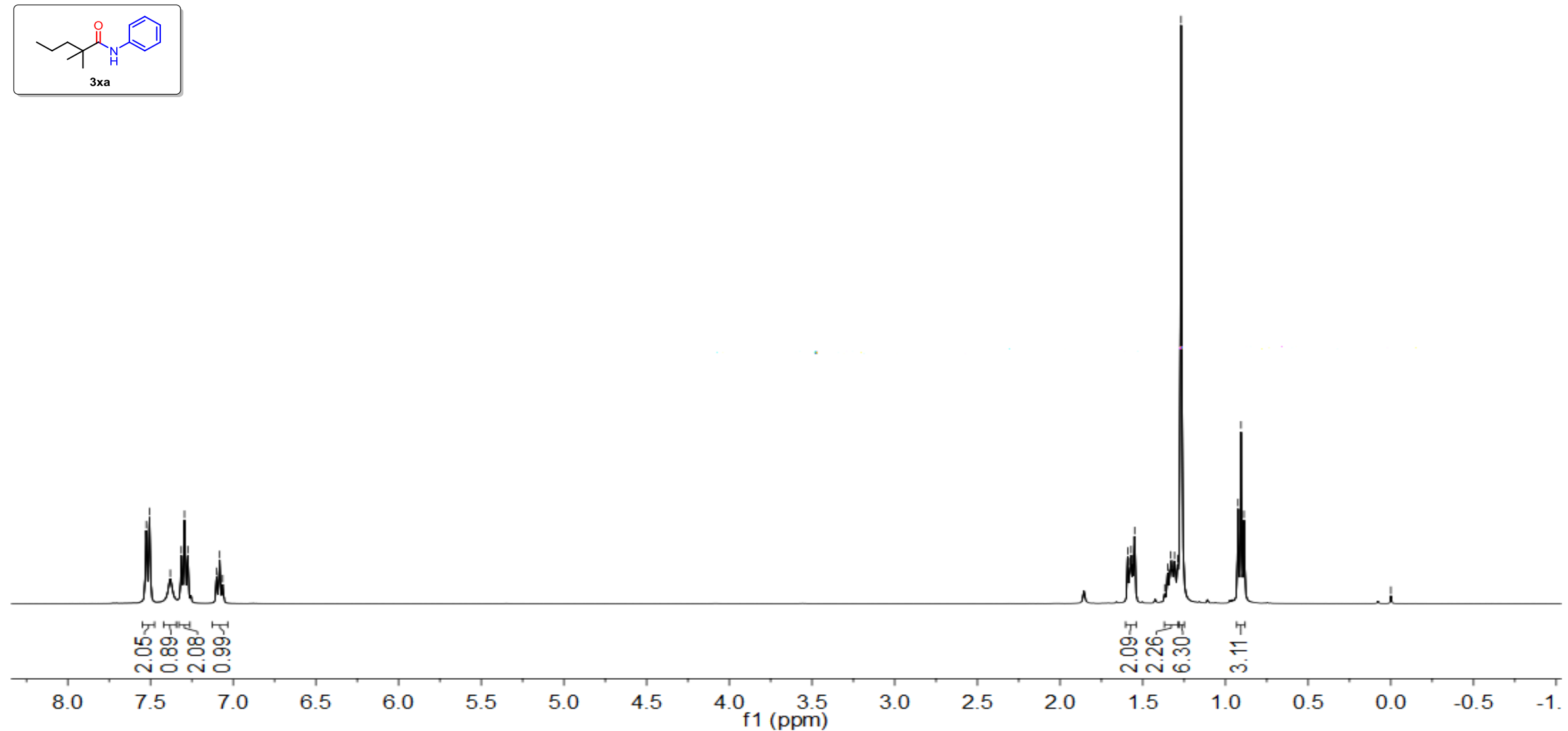
${ }^{13} \mathrm{C}$ NMR Spectra (101 MHz, $\mathrm{CDCl}_{3}$ ) of compound 3xa

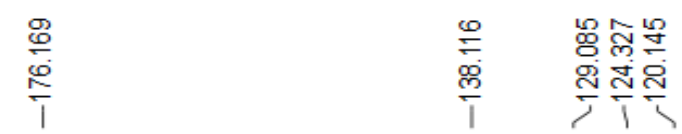

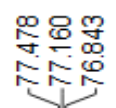

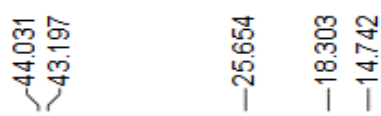
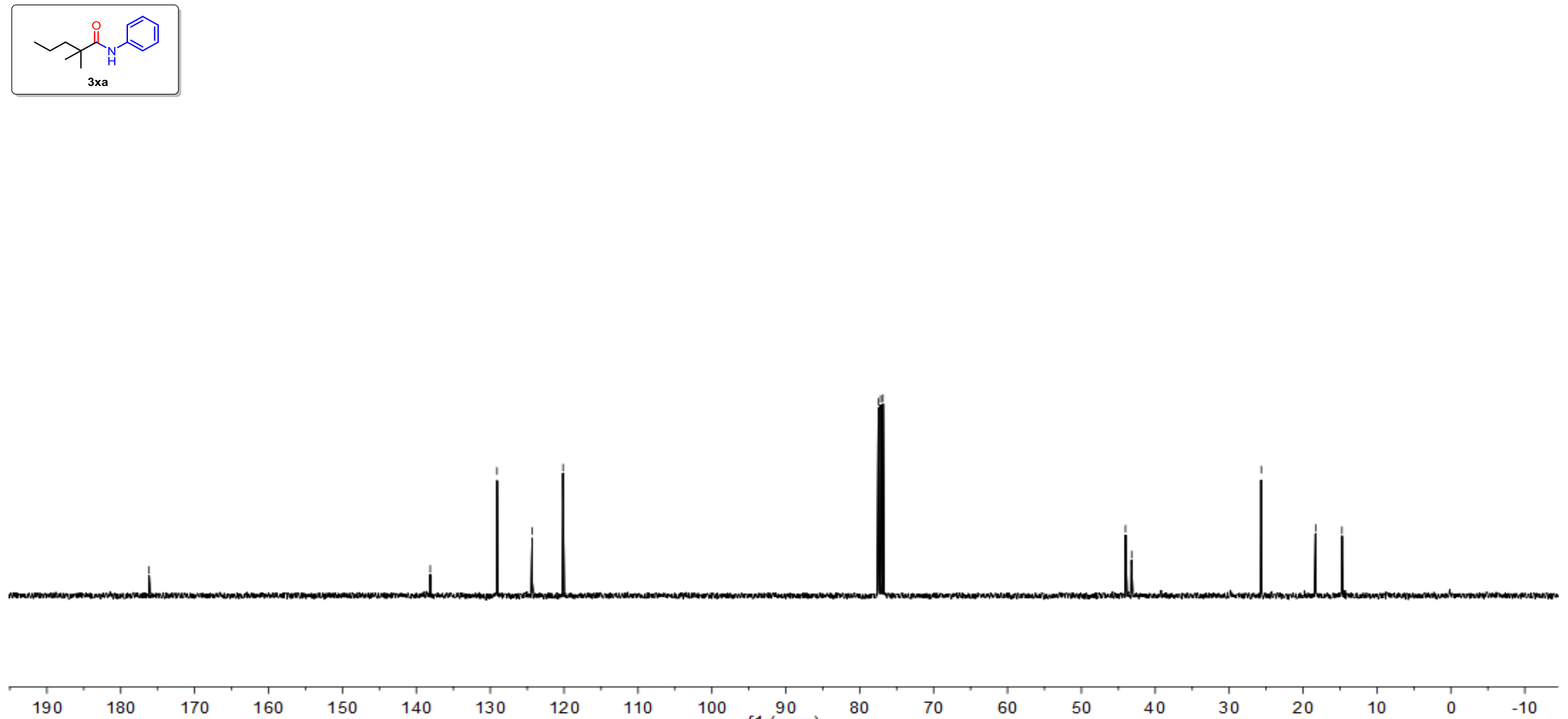

170

160

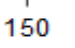

140

130

120

110

$100 \quad 900$

80

70

60

$50 \quad 40$

$30 \quad 20$ 
${ }^{1} \mathrm{H}$ NMR Spectra (400 MHz, $\mathrm{CDCl}_{3}$ ) of compound $\mathbf{4 a}$

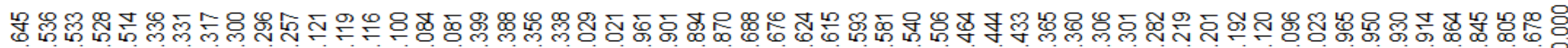

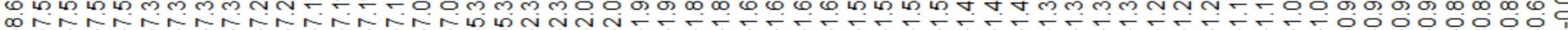
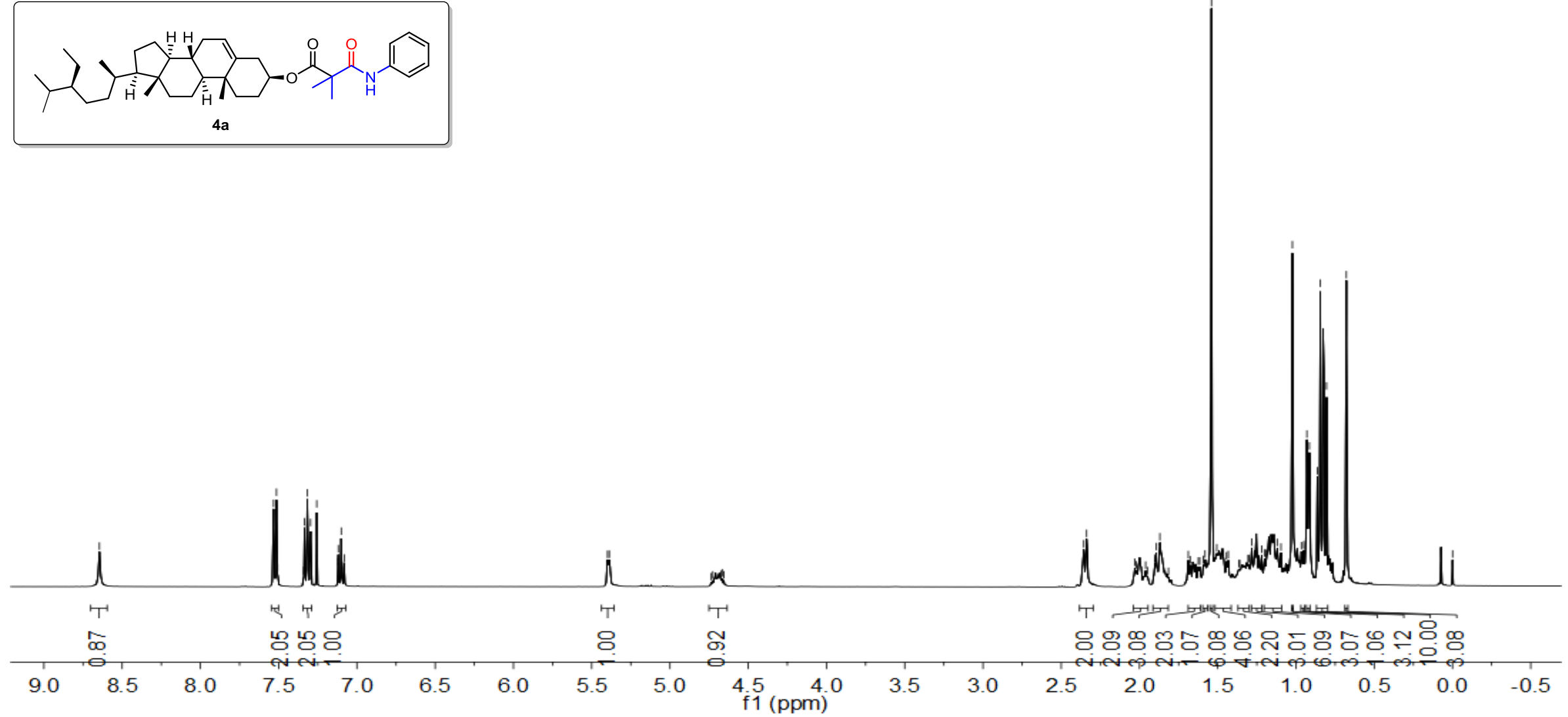
${ }^{13} \mathrm{C}$ NMR Spectra (101 MHz, $\mathrm{CDCl}_{3}$ ) of compound $4 \mathbf{a}$

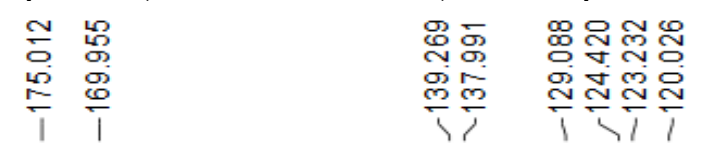

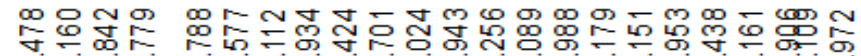

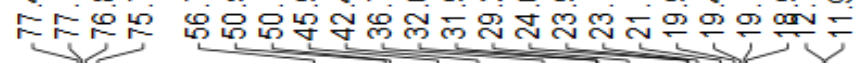
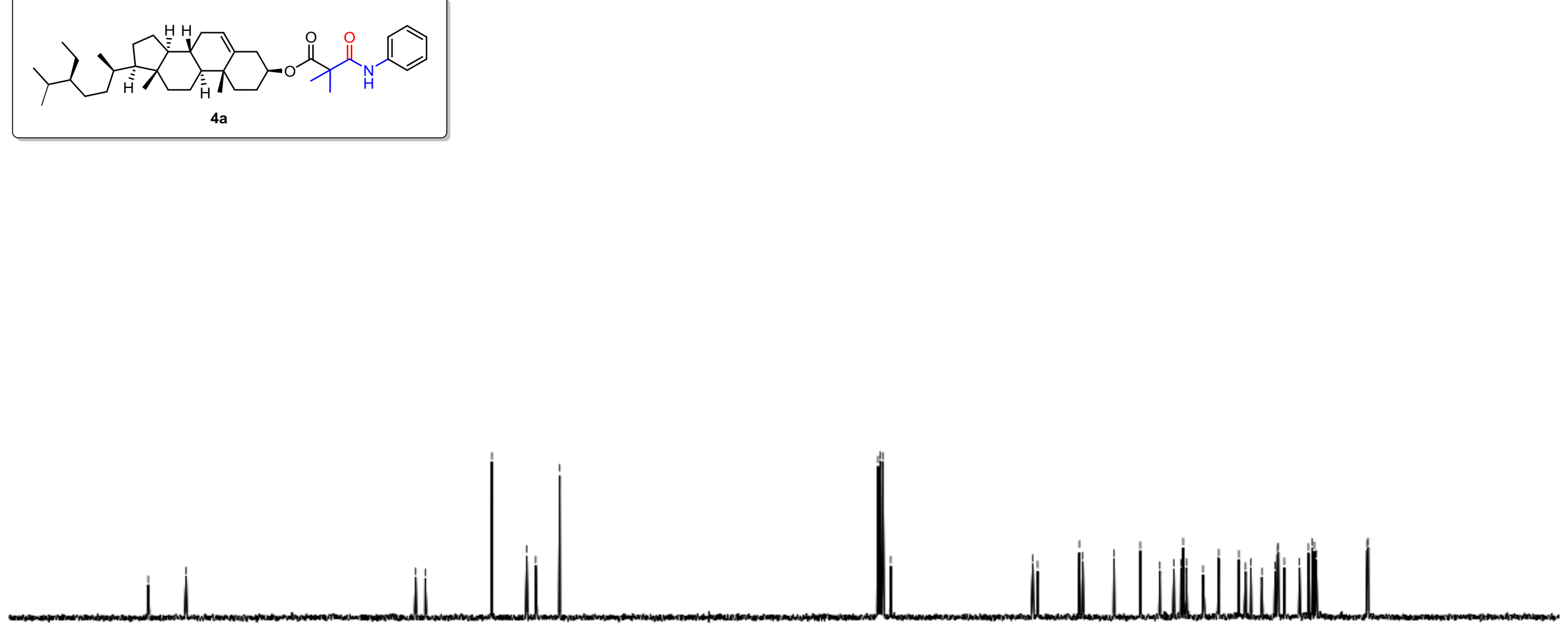

$190 \quad 180$

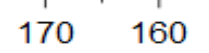

$\begin{array}{llll}150 & 140 & 130 & 120\end{array}$

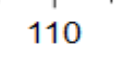

$100 \underset{\mathrm{f} 1(\mathrm{ppm})}{90} 80$ 


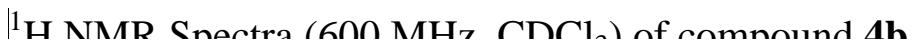

要

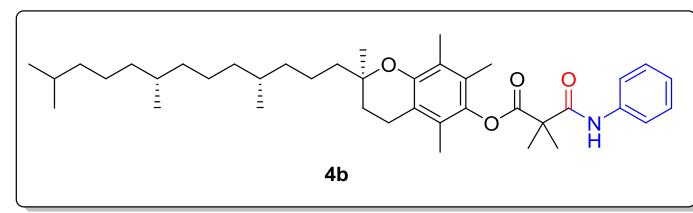

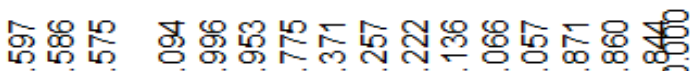 \\ 舟舟}

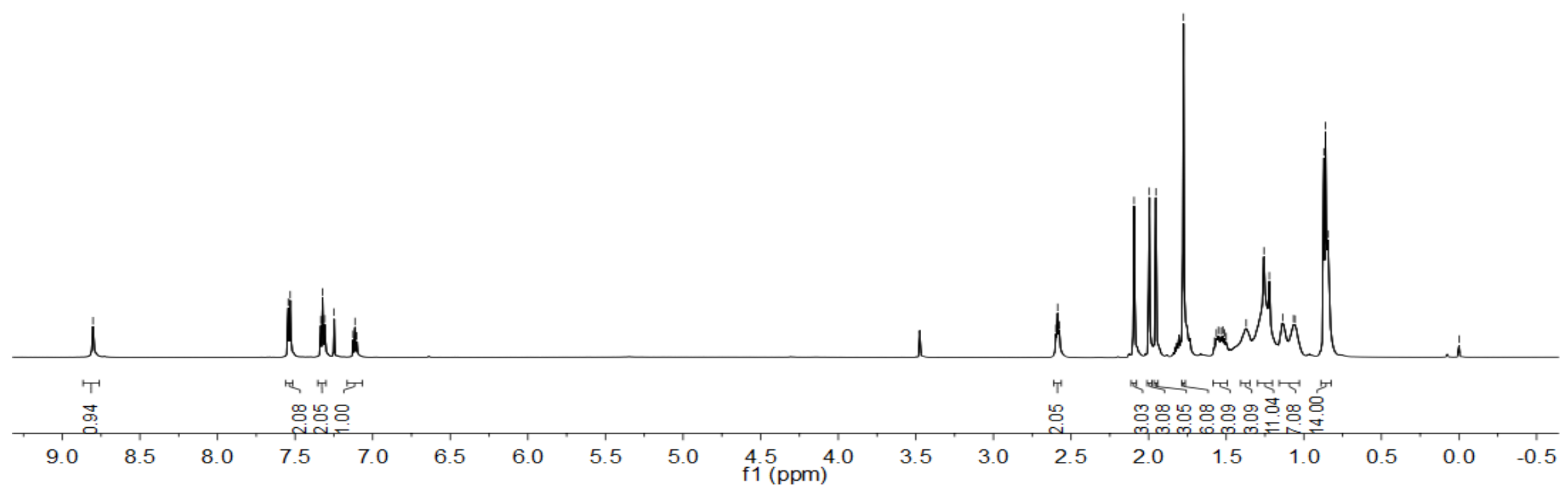


${ }^{13} \mathrm{C}$ NMR Spectra $\left(151 \mathrm{MHz}, \mathrm{CDCl}_{3}\right.$ ) of compound $\mathbf{4 b}$

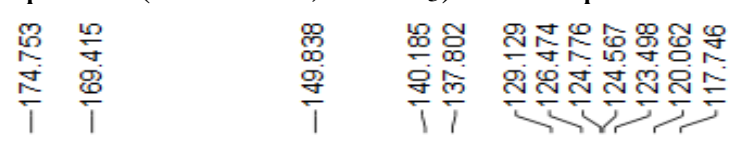

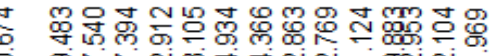

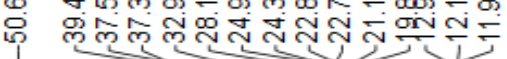
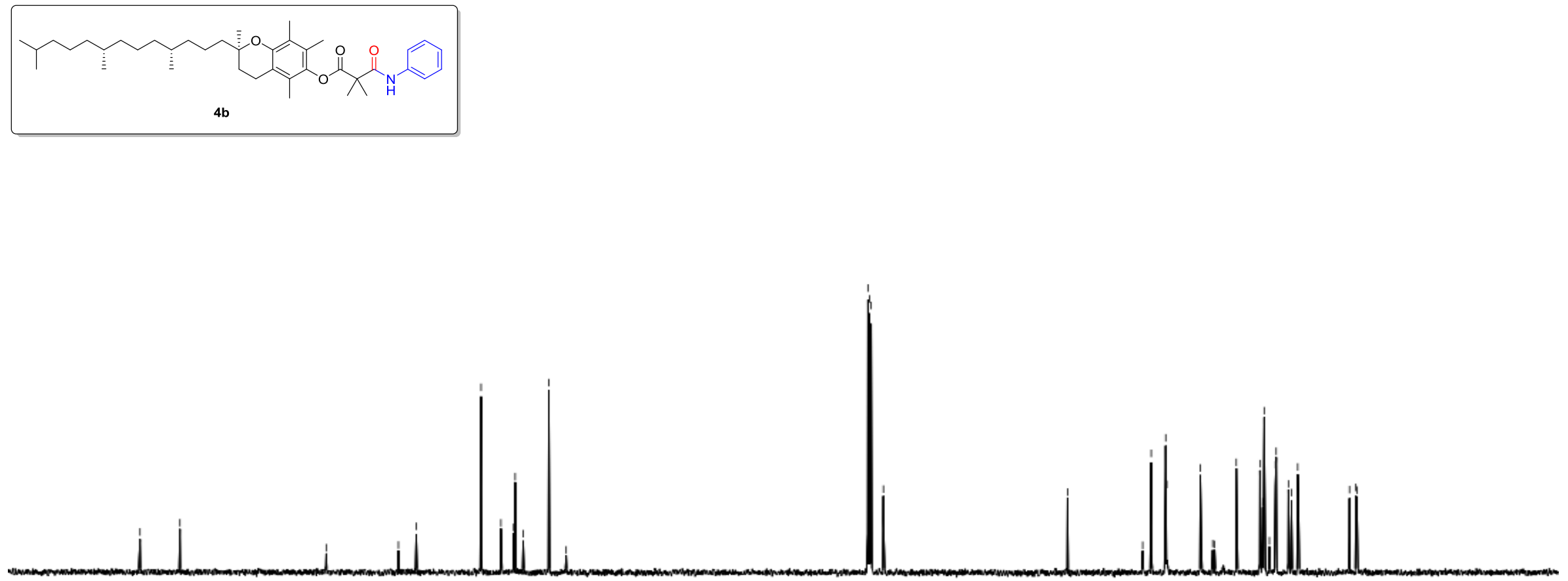

190 
${ }^{1} \mathrm{H}$ NMR Spectra (400 MHz, $\mathrm{CDCl}_{3}$ ) of compound $\mathbf{4 c}$

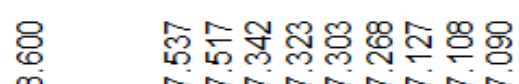

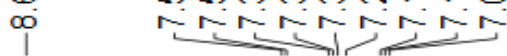

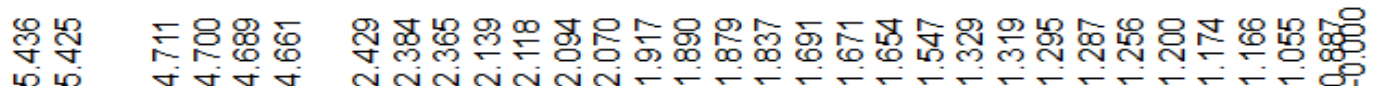

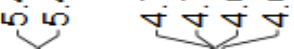

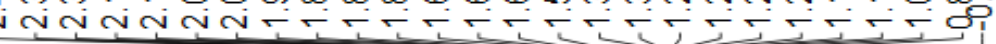
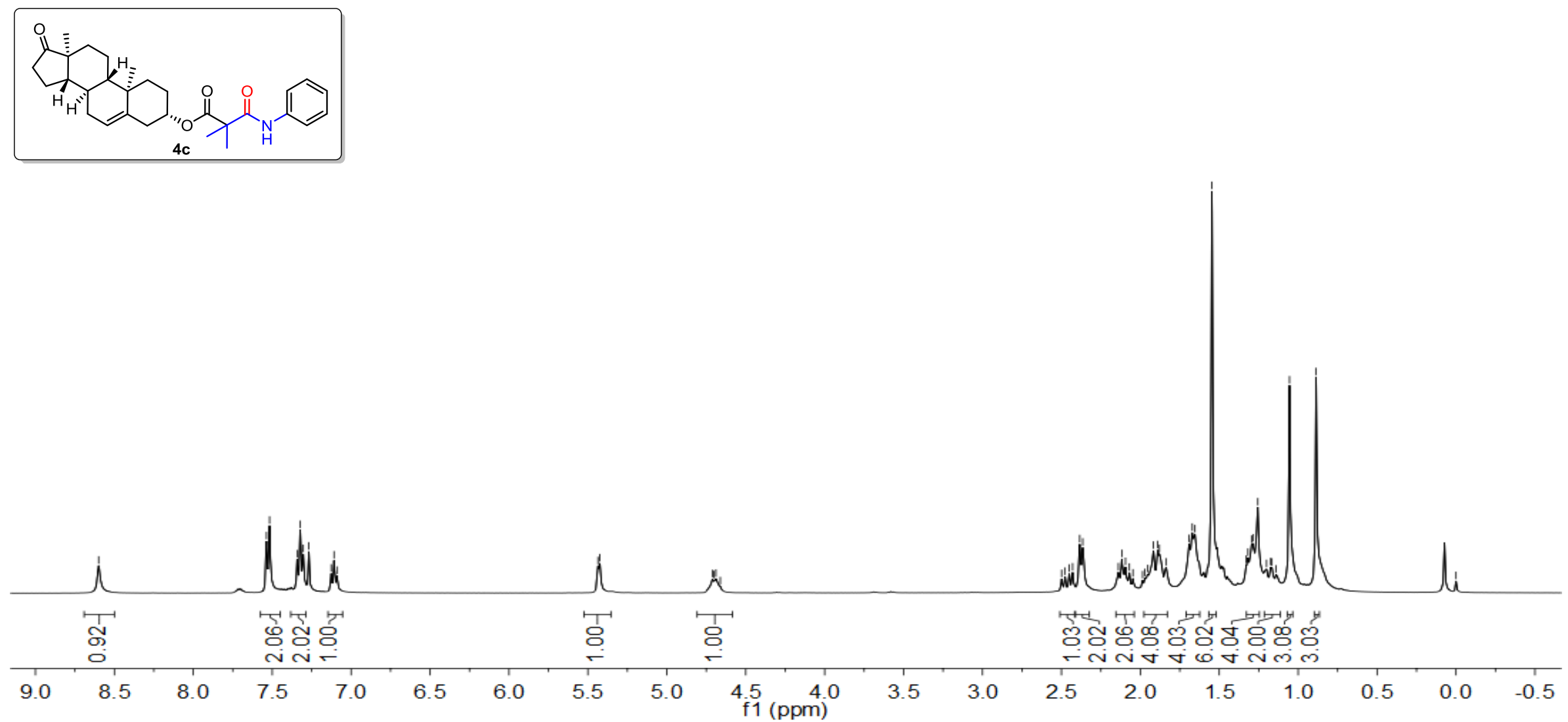
${ }^{13} \mathrm{C}$ NMR Spectra (101 MHz, $\mathrm{CDCl}_{3}$ ) of compound $\mathbf{4 c}$
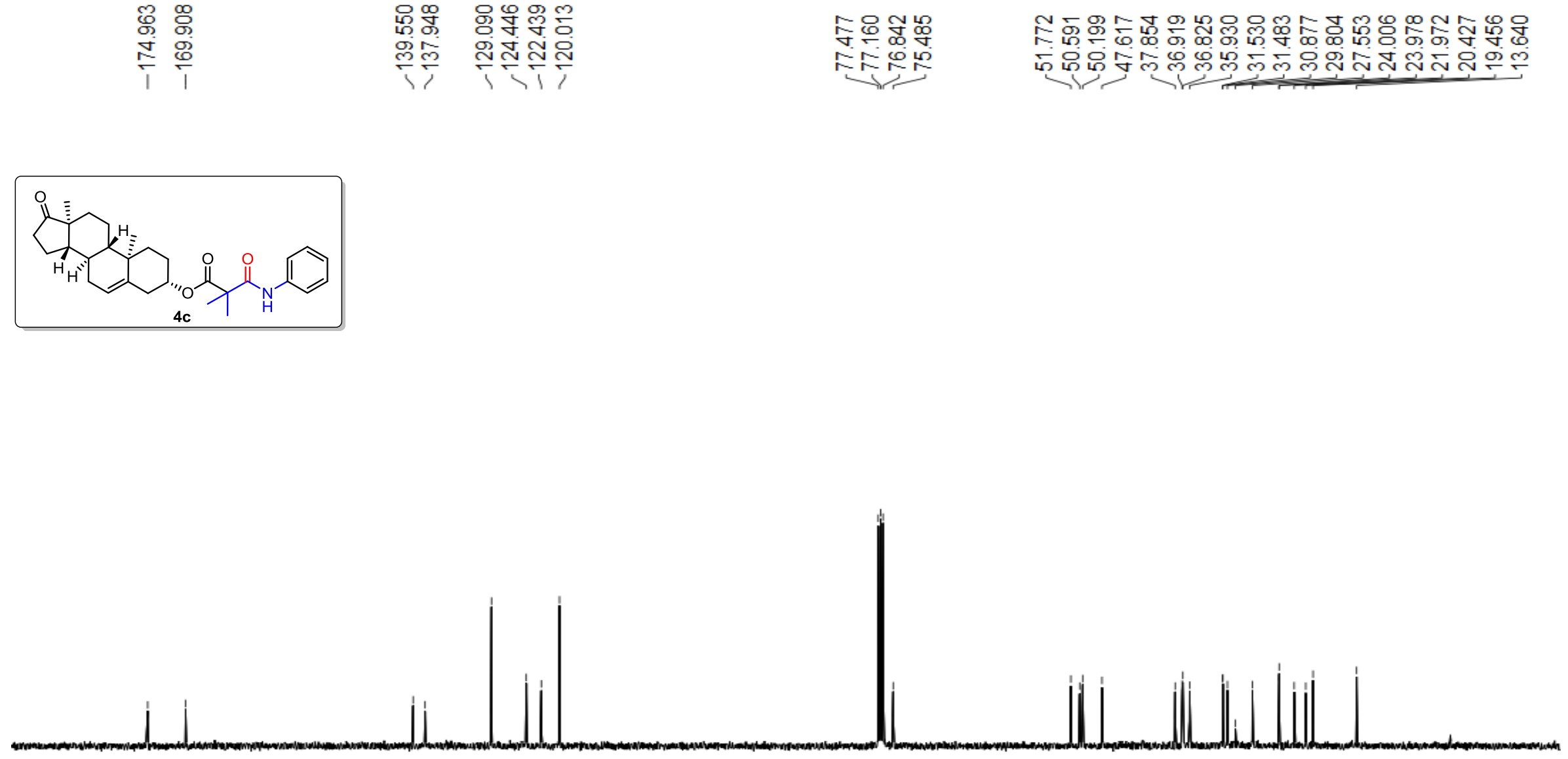
${ }^{1} \mathrm{H}$ NMR Spectra (400 MHz, $\mathrm{CDCl}_{3}$ ) of compound $\mathbf{4 d}$

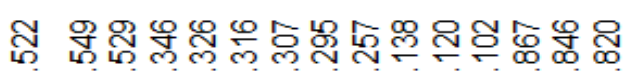

1 intratringes
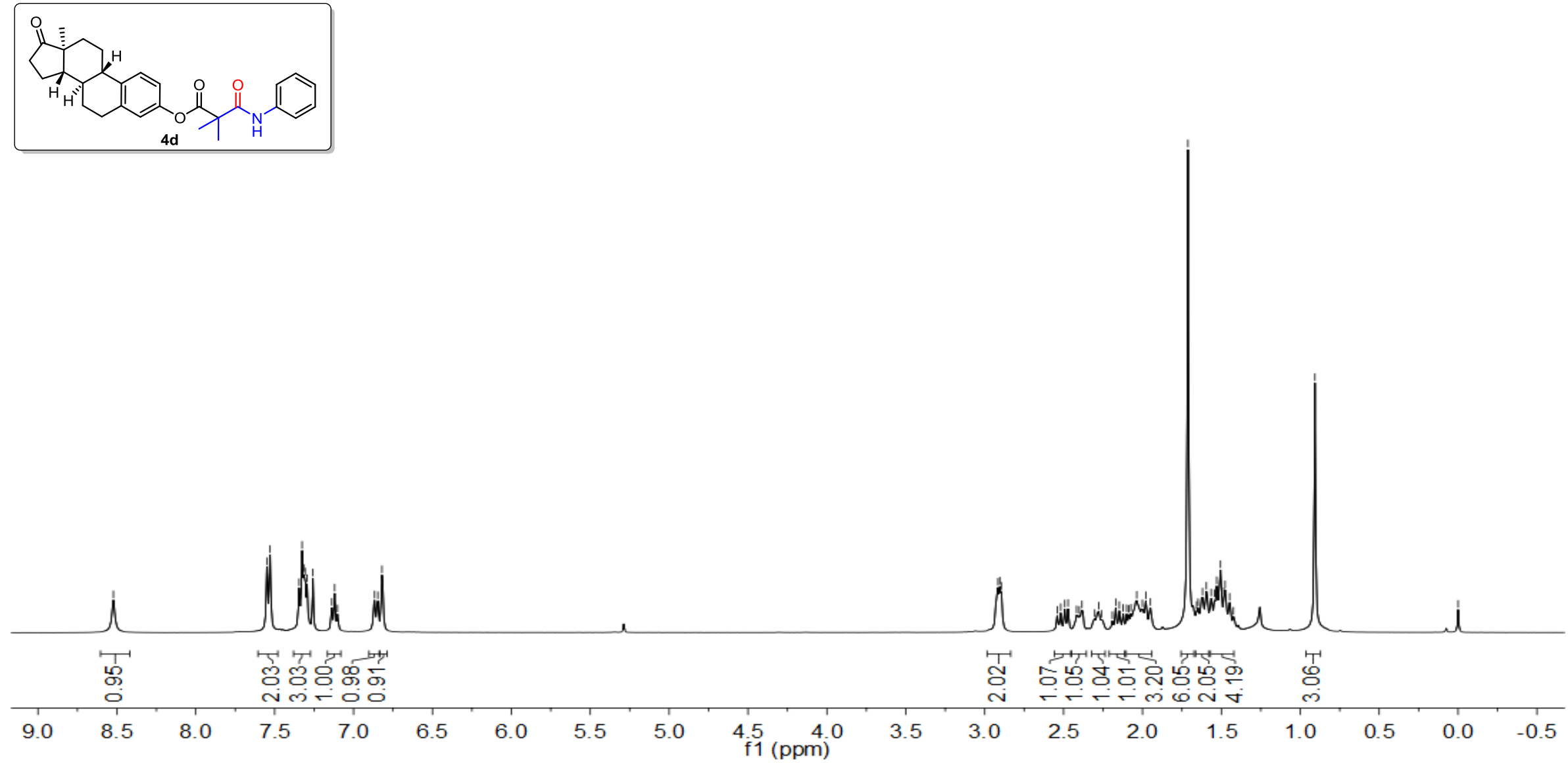
${ }^{13} \mathrm{C}$ NMR Spectra (151 $\mathrm{MHz}, \mathrm{CDCl}_{3}$ ) of compound $\mathbf{4 d}$
$\stackrel{\infty}{\infty}$
ำ

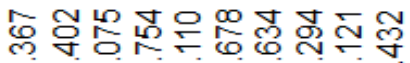
品
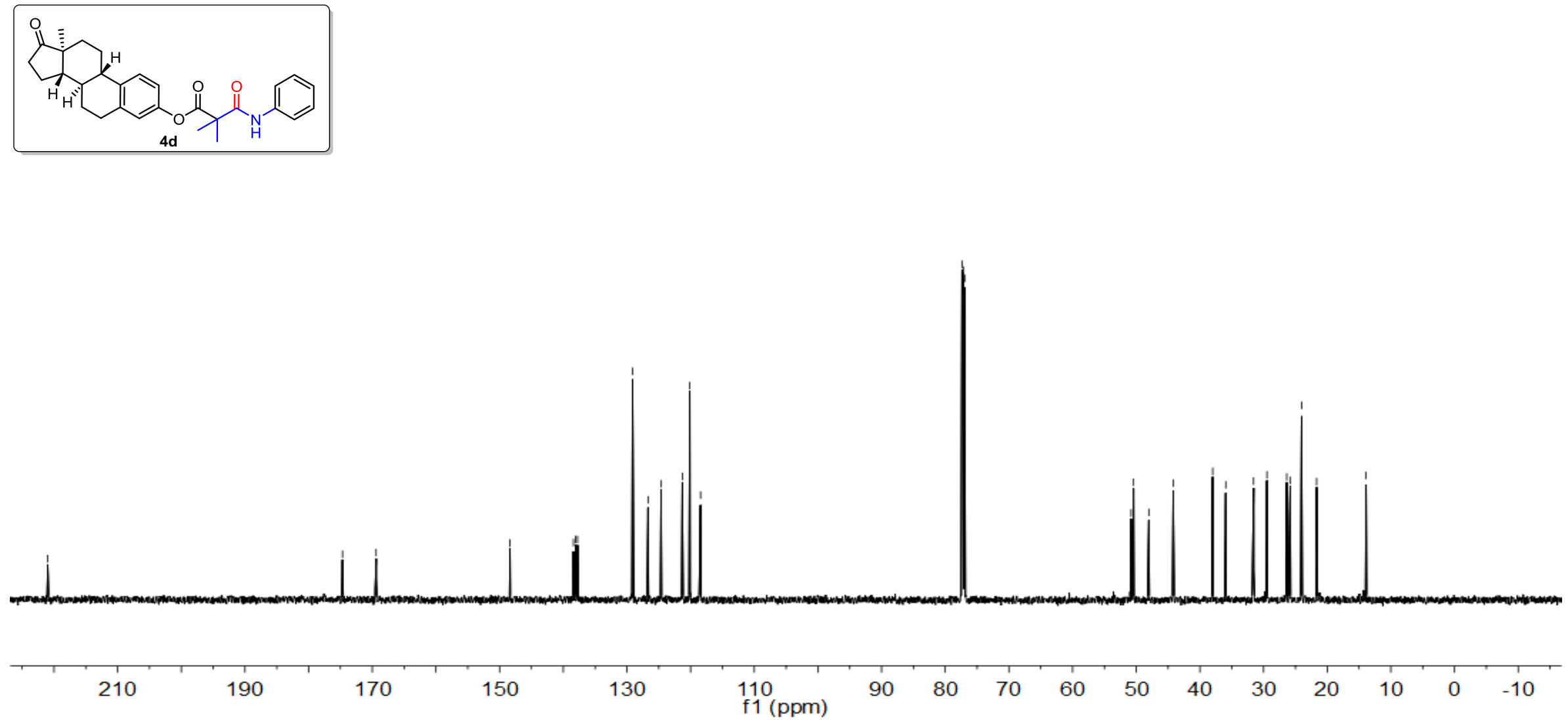
${ }^{1}$ H NMR Spectra (600 MHz, $d^{6}$-DMSO) of compound 5a

兽

事
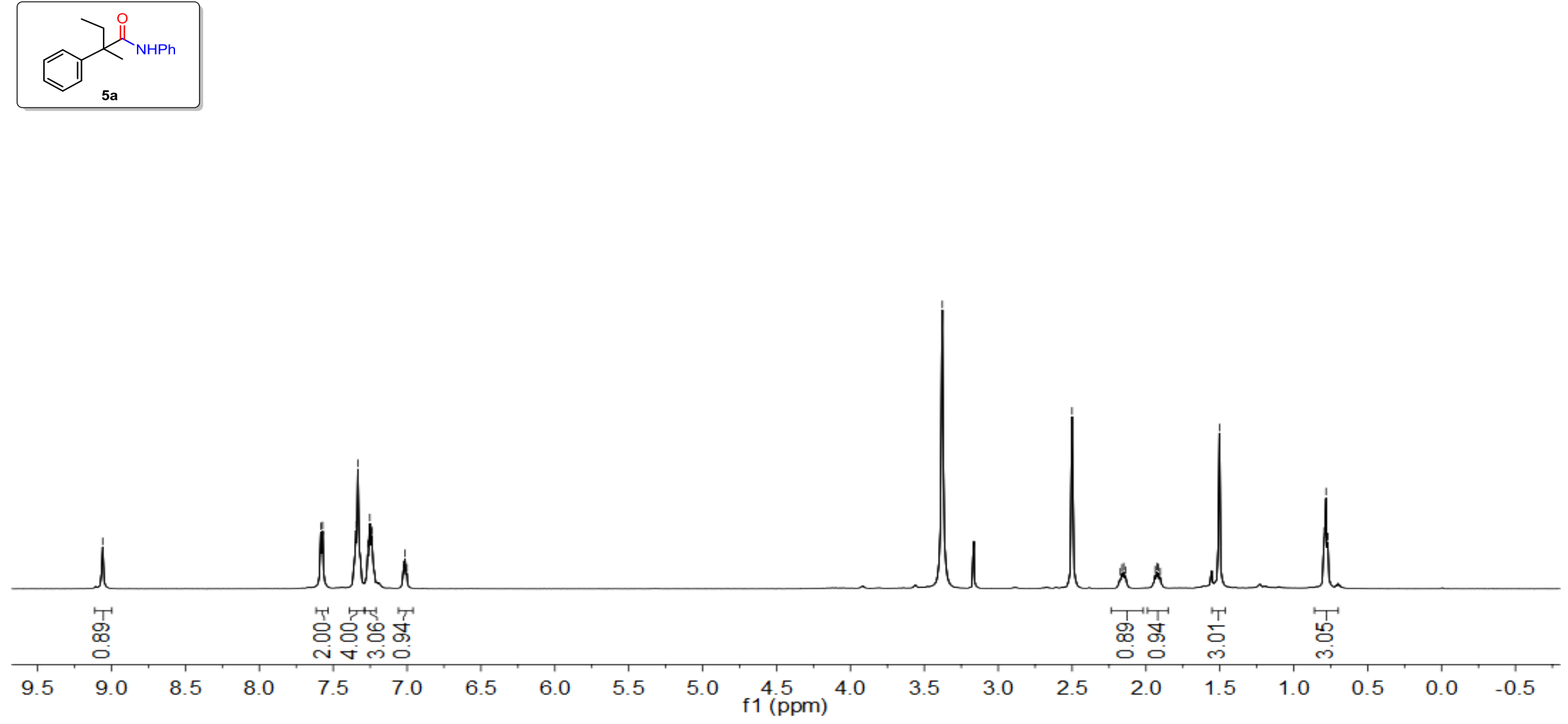
${ }^{13} \mathrm{C}$ NMR Spectra (101 MHz, $\mathrm{CDCl}_{3}$ ) of compound 5a

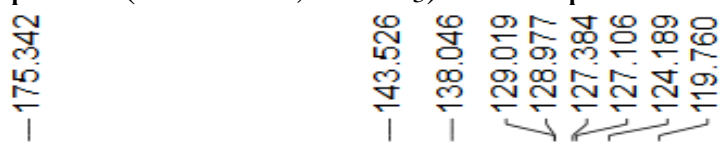

\section{ํํำ}
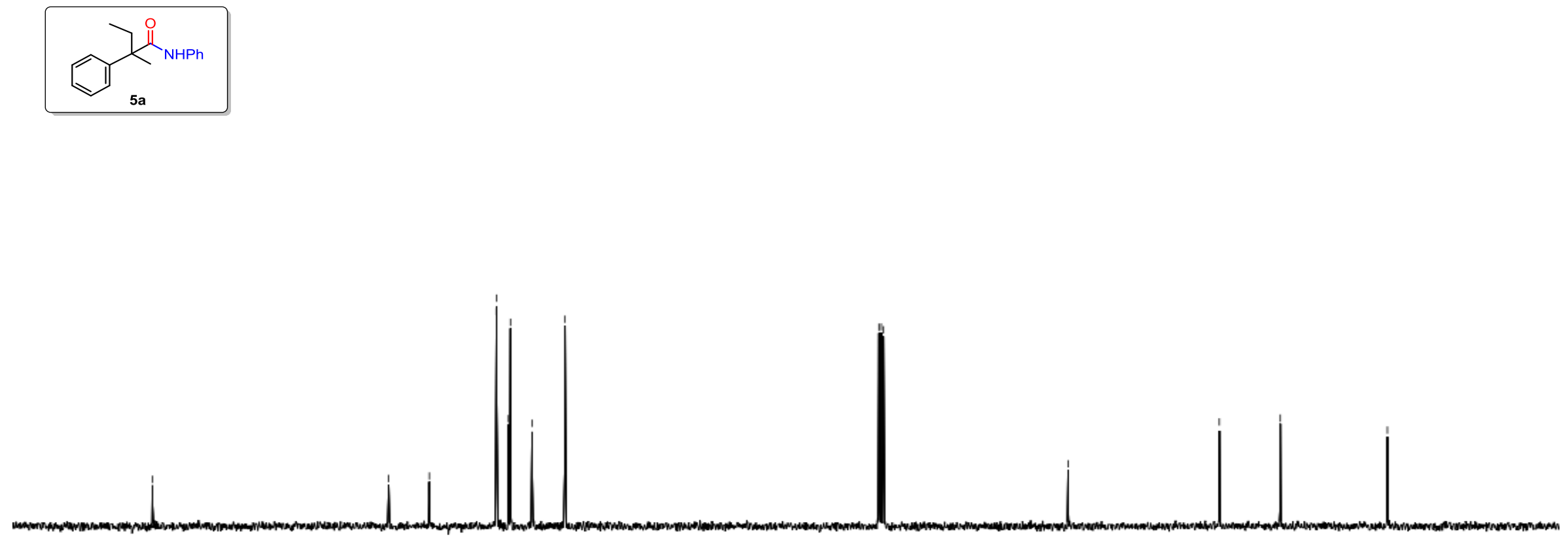

$190 \quad 180 \quad 170-160$

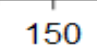

140

130

120

110

$100 \quad 90$

80

70

60

50

40

$30 \quad 20$

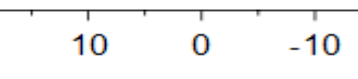


${ }^{1} \mathrm{H}$ NMR Spectra (600 MHz, DMSO- $d^{6}$ ) of compound $\mathbf{5 b}$

O.

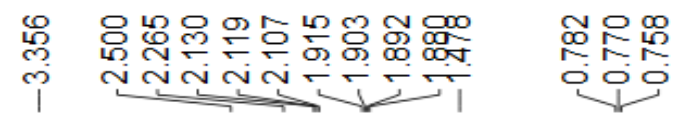
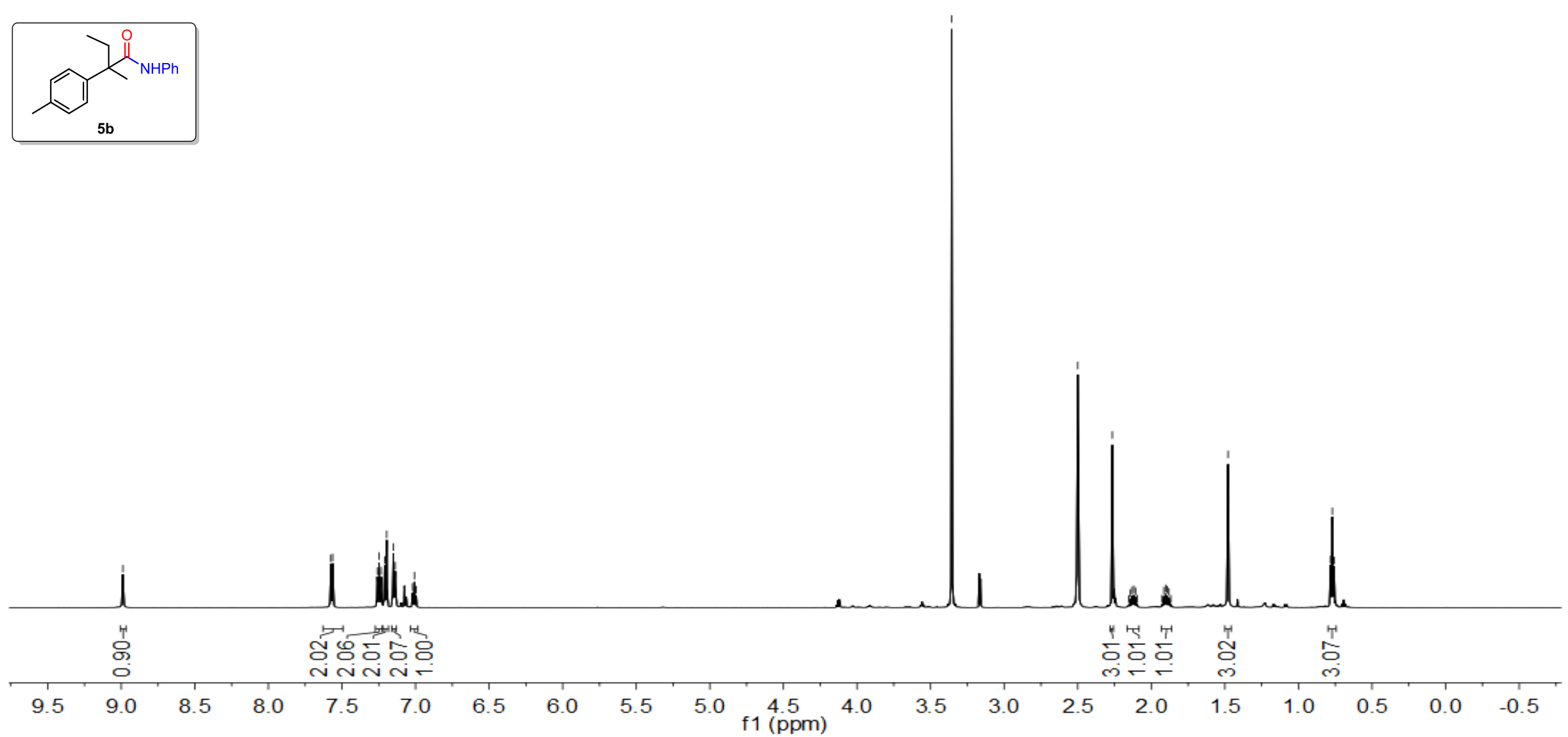
${ }^{13} \mathrm{C}$ NMR Spectra (151 MHz, DMSO- $d^{6}$ ) of compound $\mathbf{5 b}$

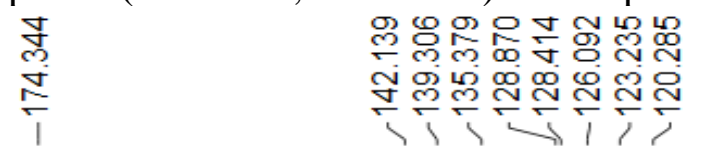

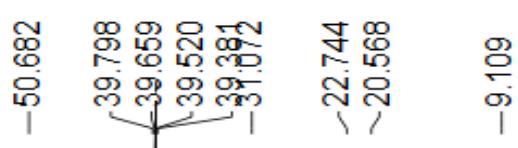

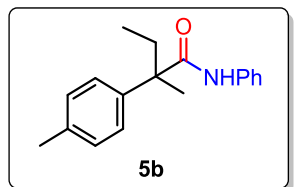

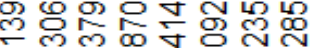

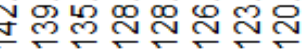




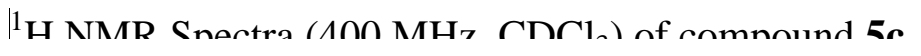

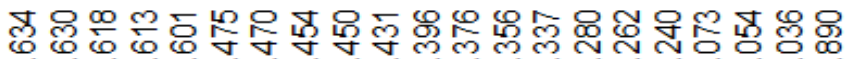

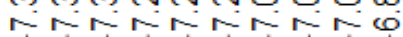

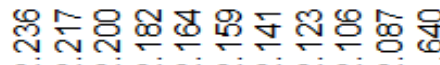

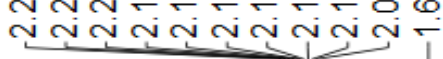

\section{適离}

io

응
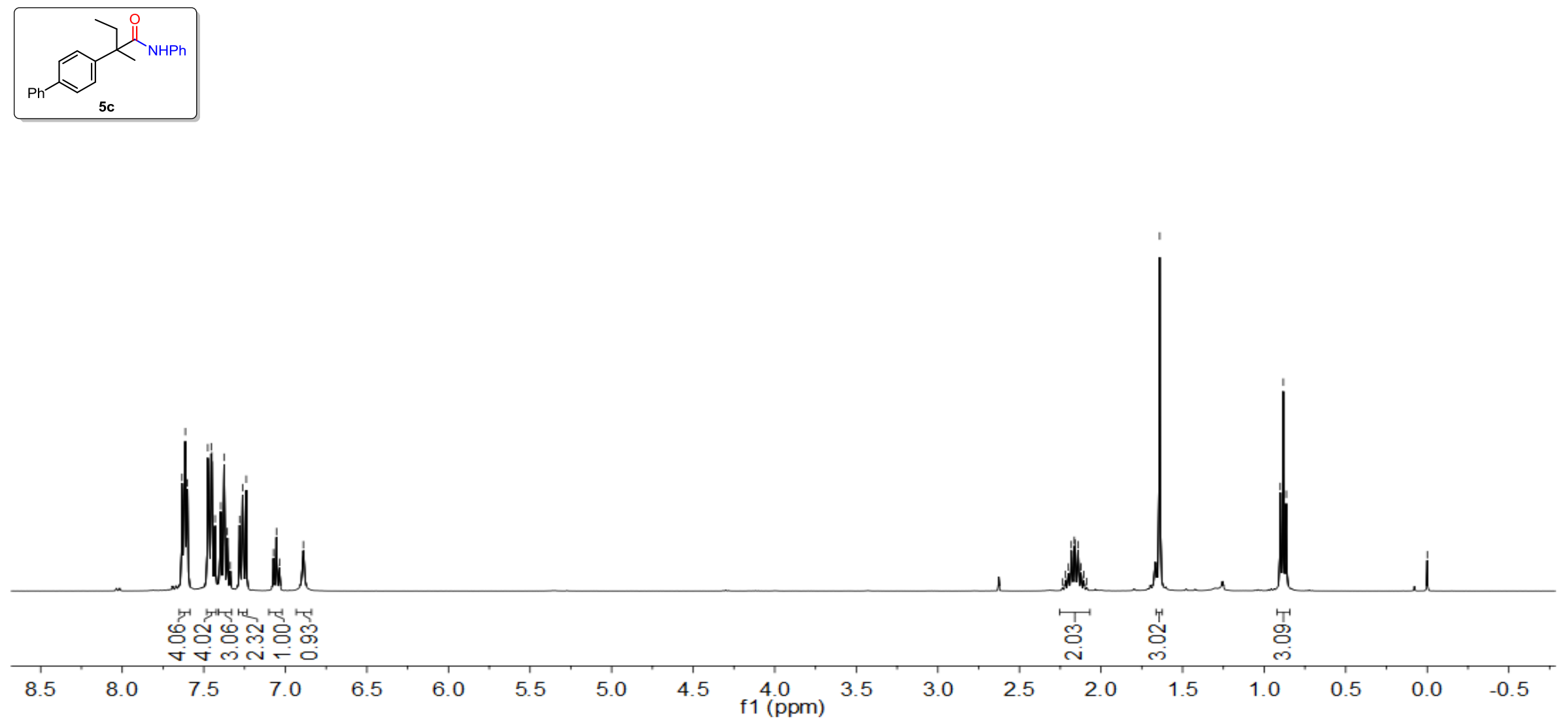
${ }^{13} \mathrm{C}$ NMR Spectra $\left(101 \mathrm{MHz}, \mathrm{CDCl}_{3}\right.$ ) of compound $\mathbf{5 c}$

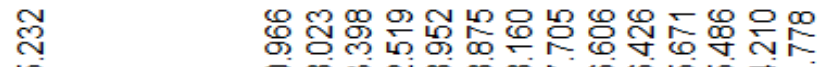

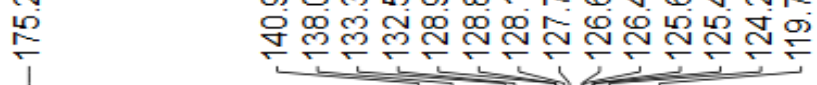

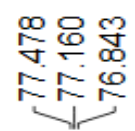

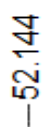

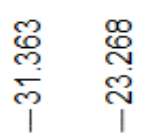

$\underset{\substack{\infty \\ \infty}}{\infty}$
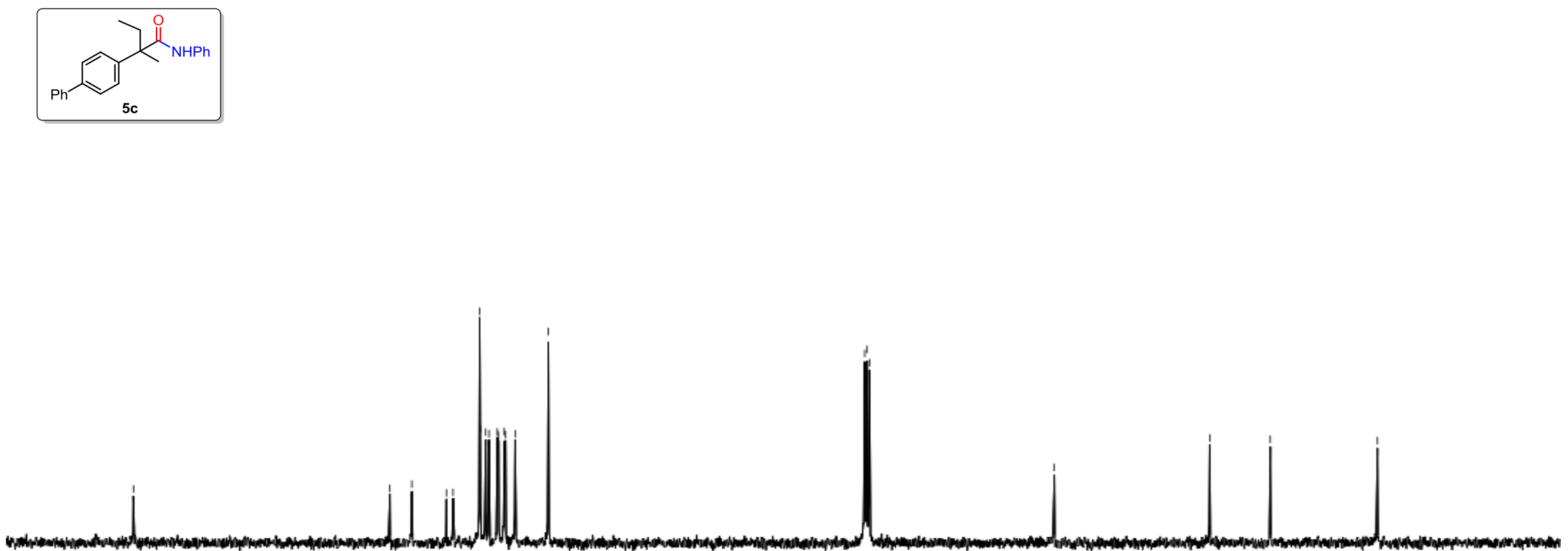
${ }^{1} \mathrm{H}$ NMR Spectra (400 MHz, $\mathrm{CDCl}_{3}$ ) of compound 5d

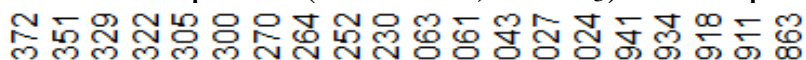

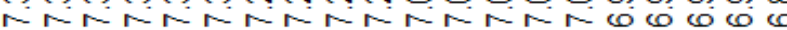
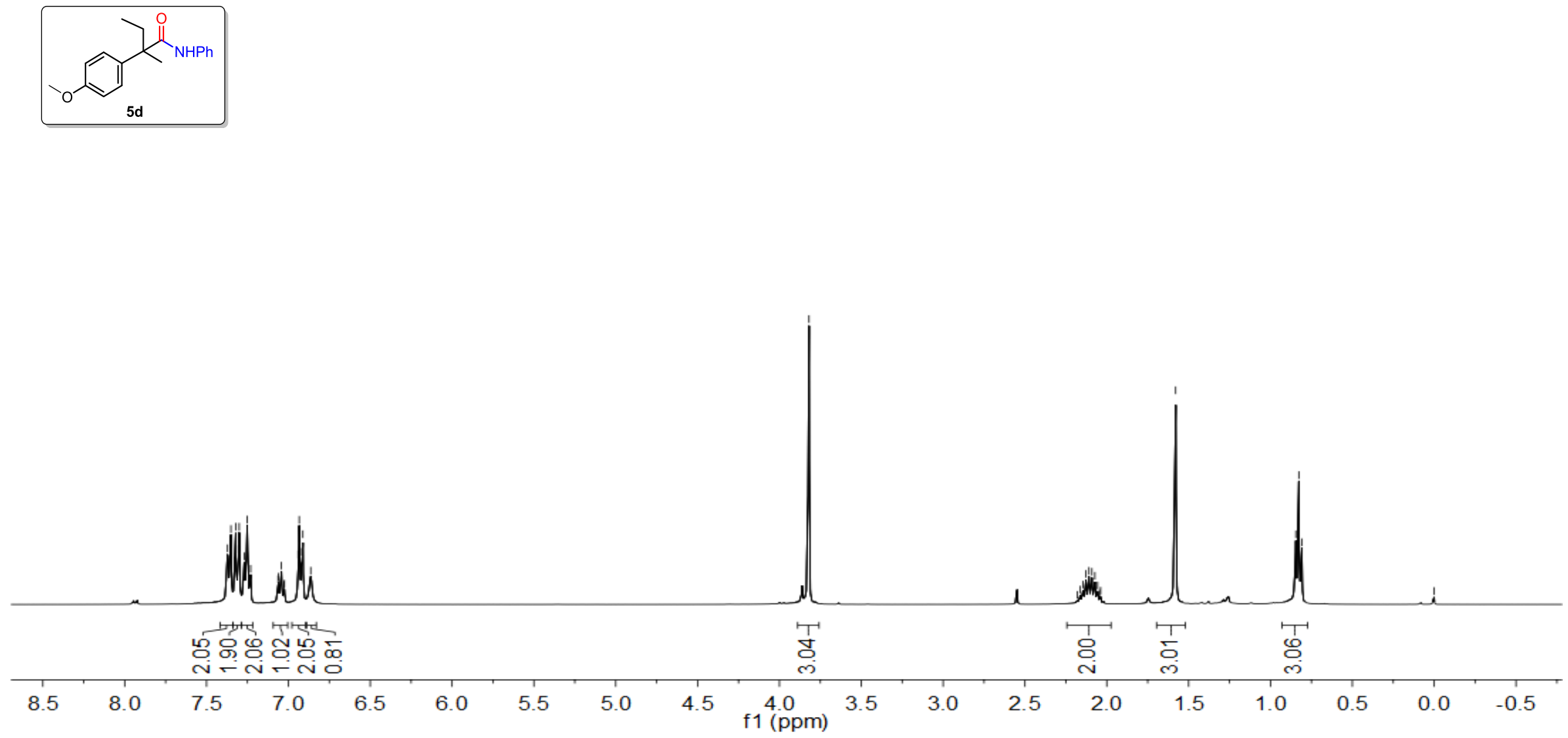
${ }^{13} \mathrm{C}$ NMR Spectra $\left(101 \mathrm{MHz}, \mathrm{CDCl}_{3}\right)$ of compound $\mathbf{5 d}$

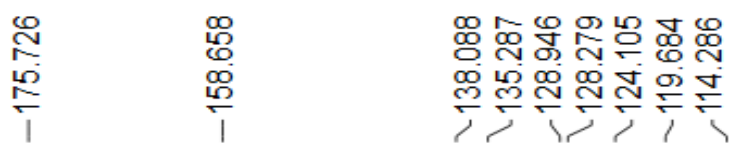

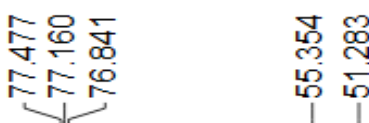

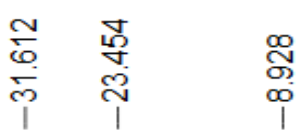
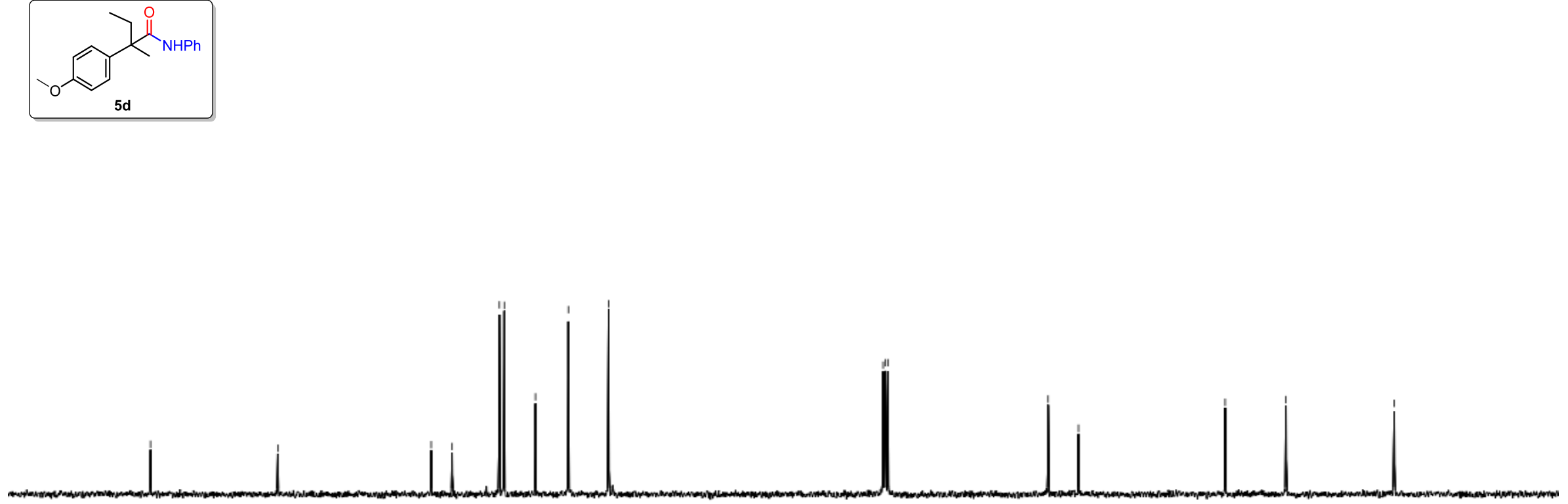

$\begin{array}{llll}190 & 180 & 170 & 160\end{array}$

110

$00 \quad 90$

$80-70$

$60 \quad 50$

$40 \quad 30$

$20 \quad 10 \cdot 0 \cdot \frac{1}{1}$

S155 
${ }^{1} \mathrm{H}$ NMR Spectra (400 MHz, $\mathrm{CDCl}_{3}$ ) of compound $\mathbf{5 e}$

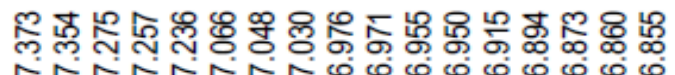

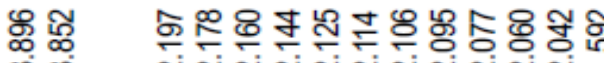

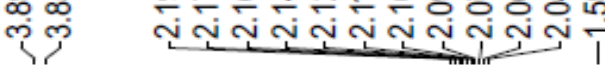

宓罚

@̊̀.
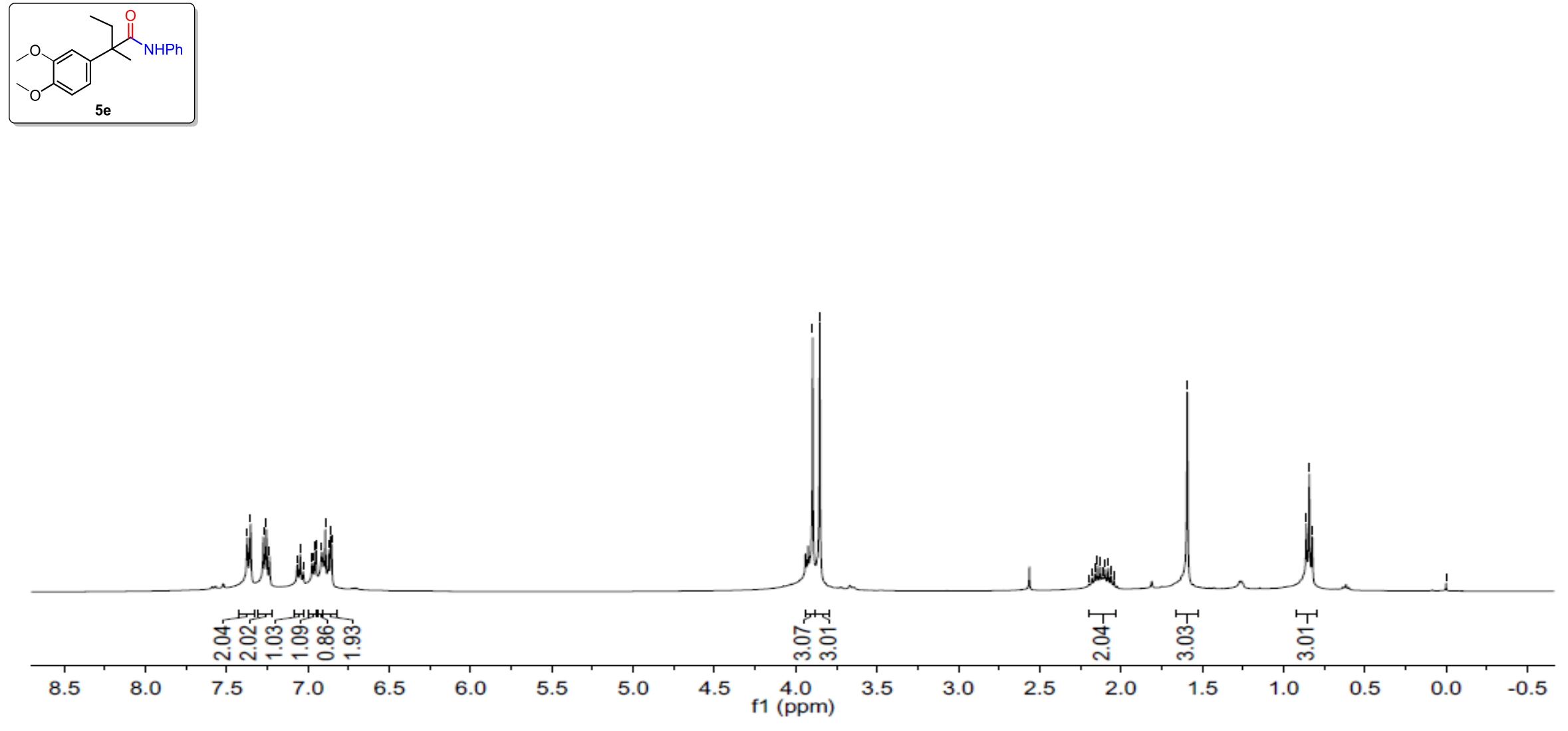
${ }^{13} \mathrm{C}$ NMR Spectra $\left(101 \mathrm{MHz}, \mathrm{CDCl}_{3}\right)$ of compound $\mathbf{5 e}$

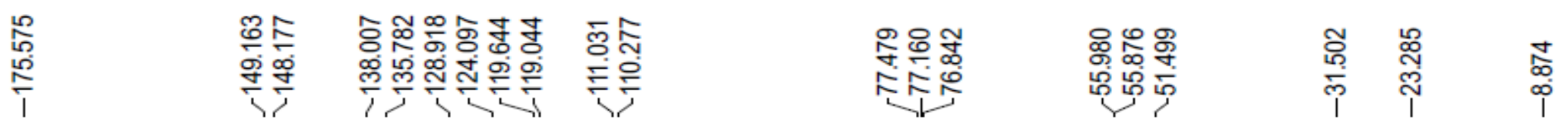
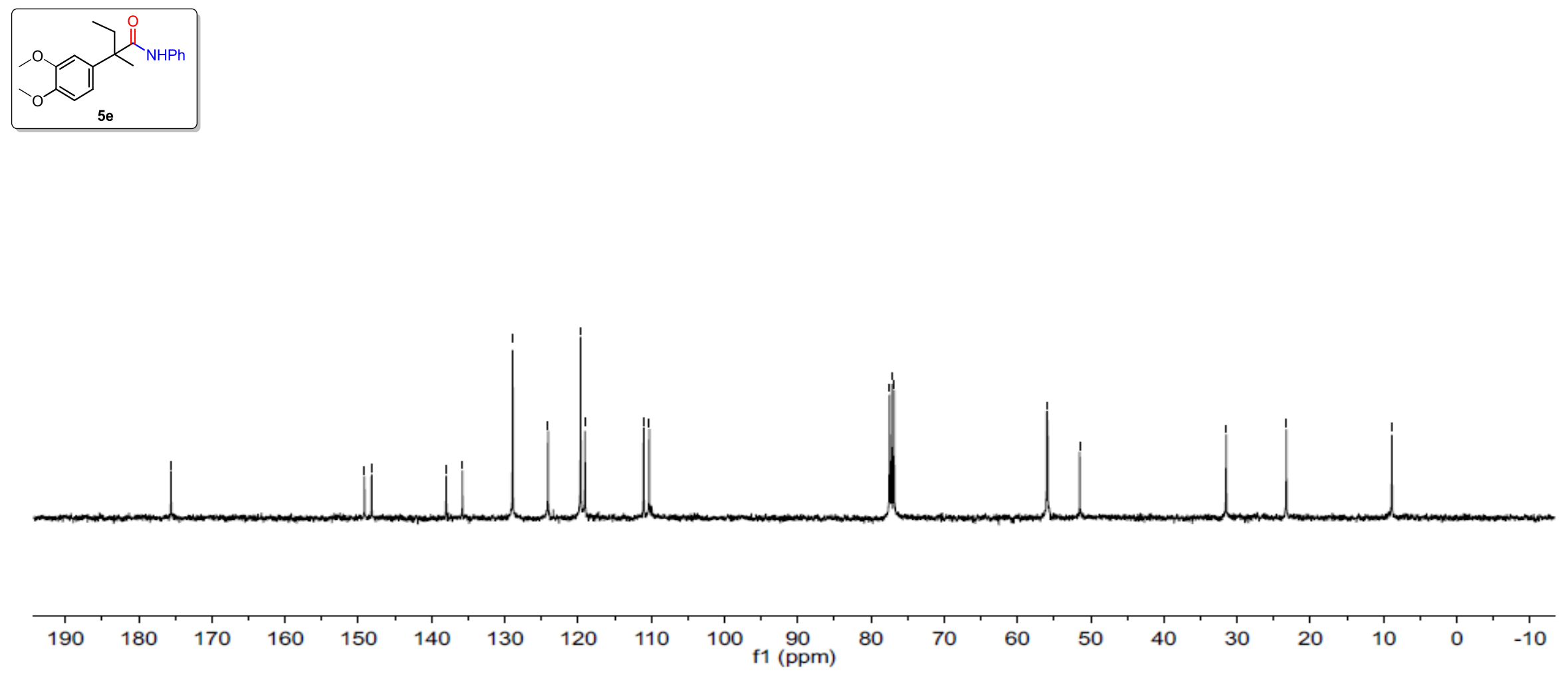
${ }^{1} \mathrm{H}$ NMR Spectra (400 MHz, $\mathrm{CDCl}_{3}$ ) of compound $\mathbf{5 f}$

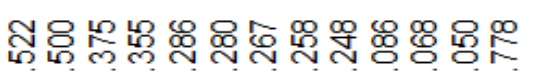

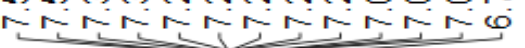
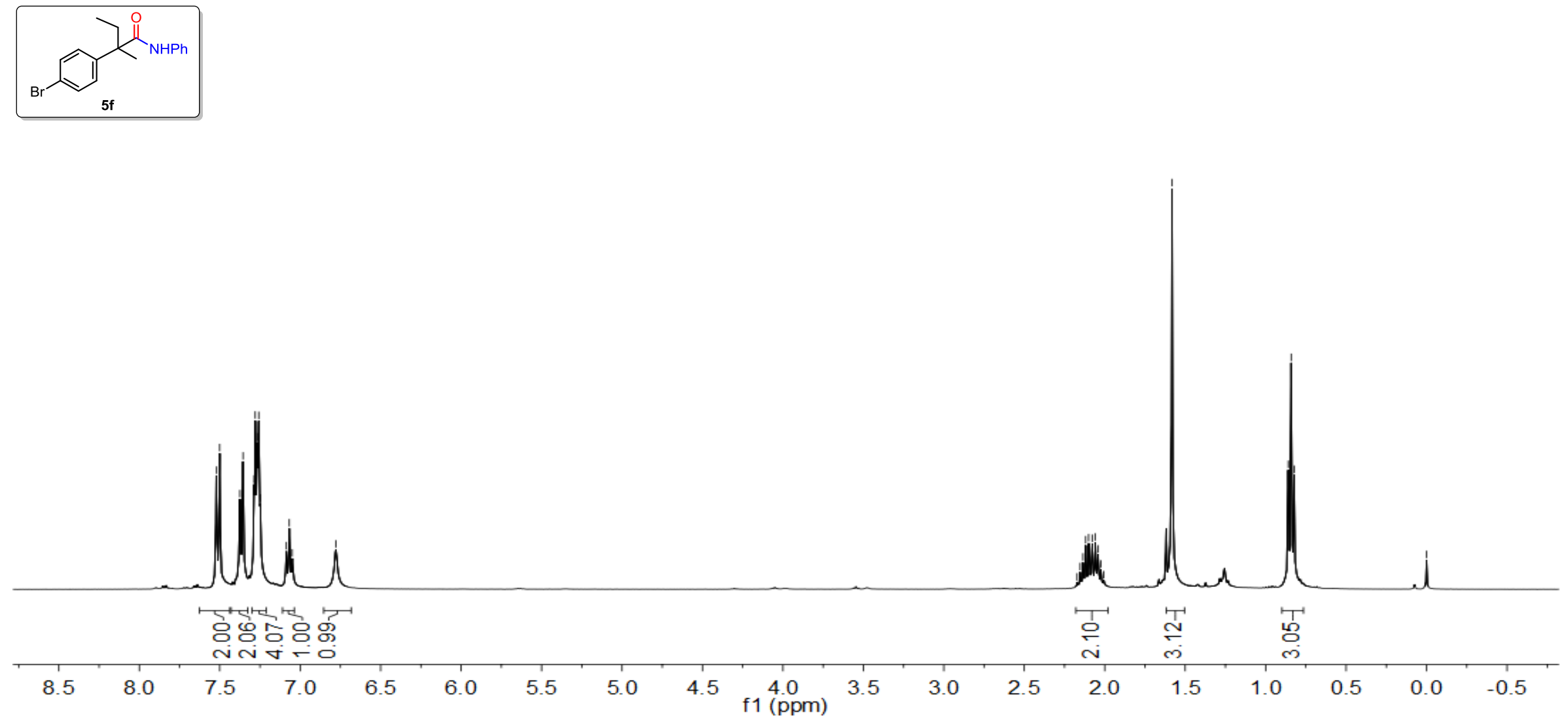
${ }^{13} \mathrm{C}$ NMR Spectra $\left(101 \mathrm{MHz}, \mathrm{CDCl}_{3}\right.$ ) of compound $\mathbf{5 f}$

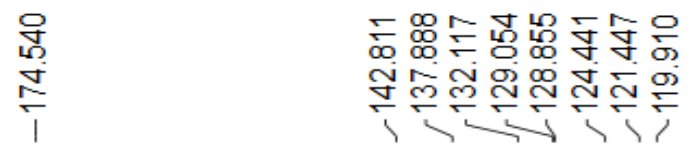

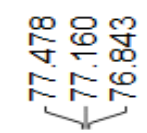
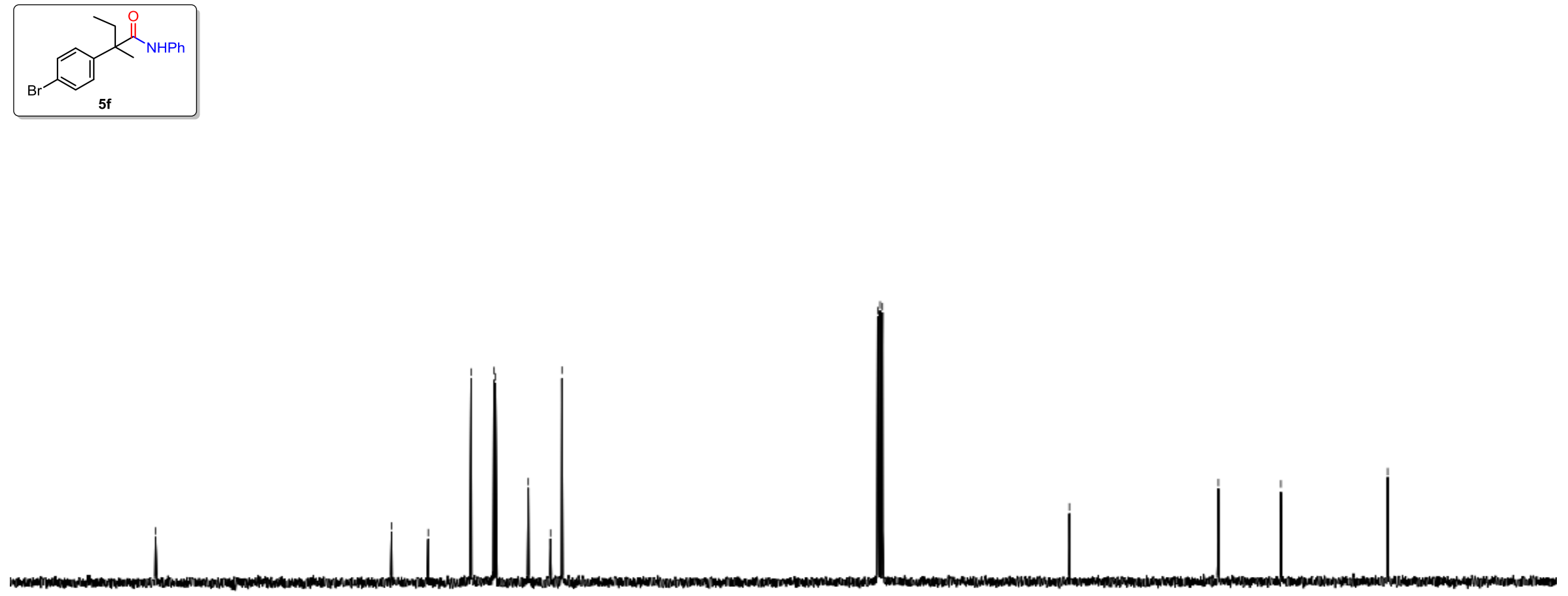

$190 \quad 180 \quad 170$ 
${ }^{1} \mathrm{H}$ NMR Spectra (400 MHz, $\mathrm{CDCl}_{3}$ ) of compound $\mathbf{5 g}$

태 ळ.

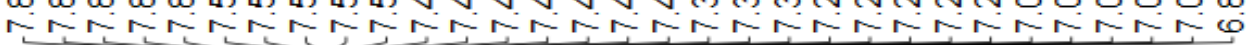
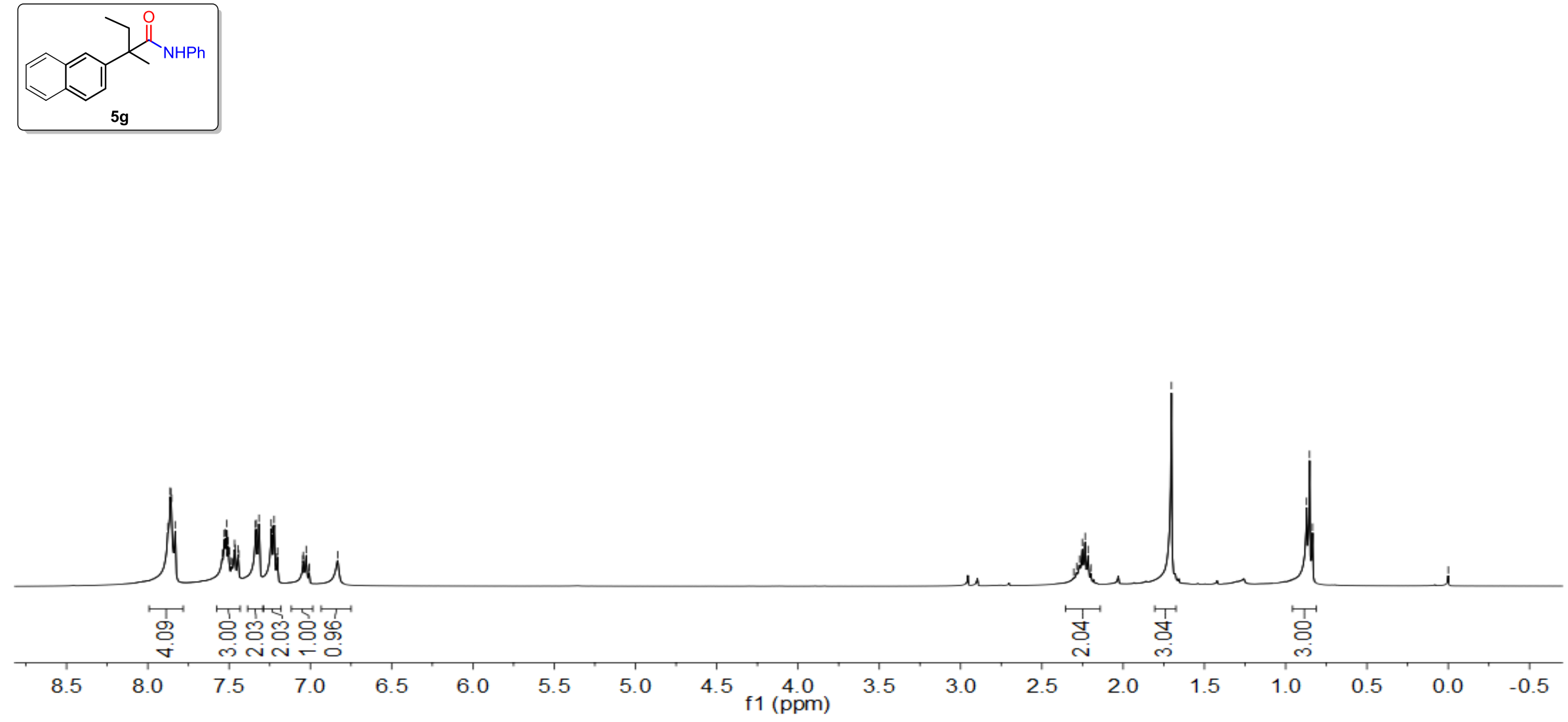
${ }^{13} \mathrm{C}$ NMR Spectra (101 MHz, $\mathrm{CDCl}_{3}$ ) of compound $\mathbf{5 g}$

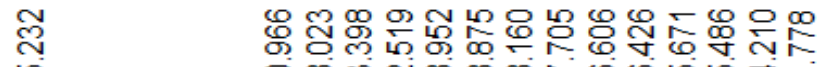

: $\quad$ 年

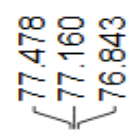

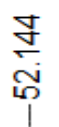

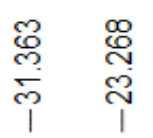

$\underset{\substack{\infty \\ \infty}}{\infty}$

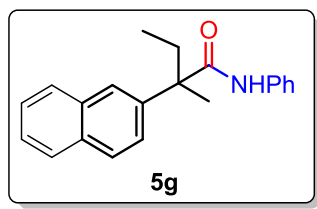

(1)

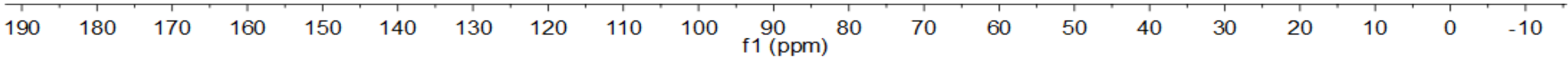


${ }^{1} \mathrm{H}$ NMR Spectra (400 MHz, $\mathrm{CDCl}_{3}$ ) of compound $\mathbf{5 h}$

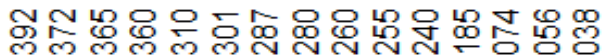

minn

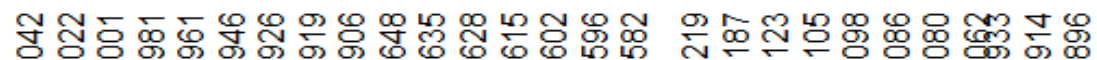

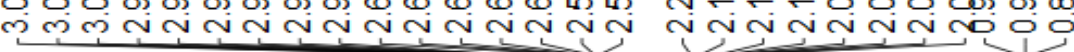
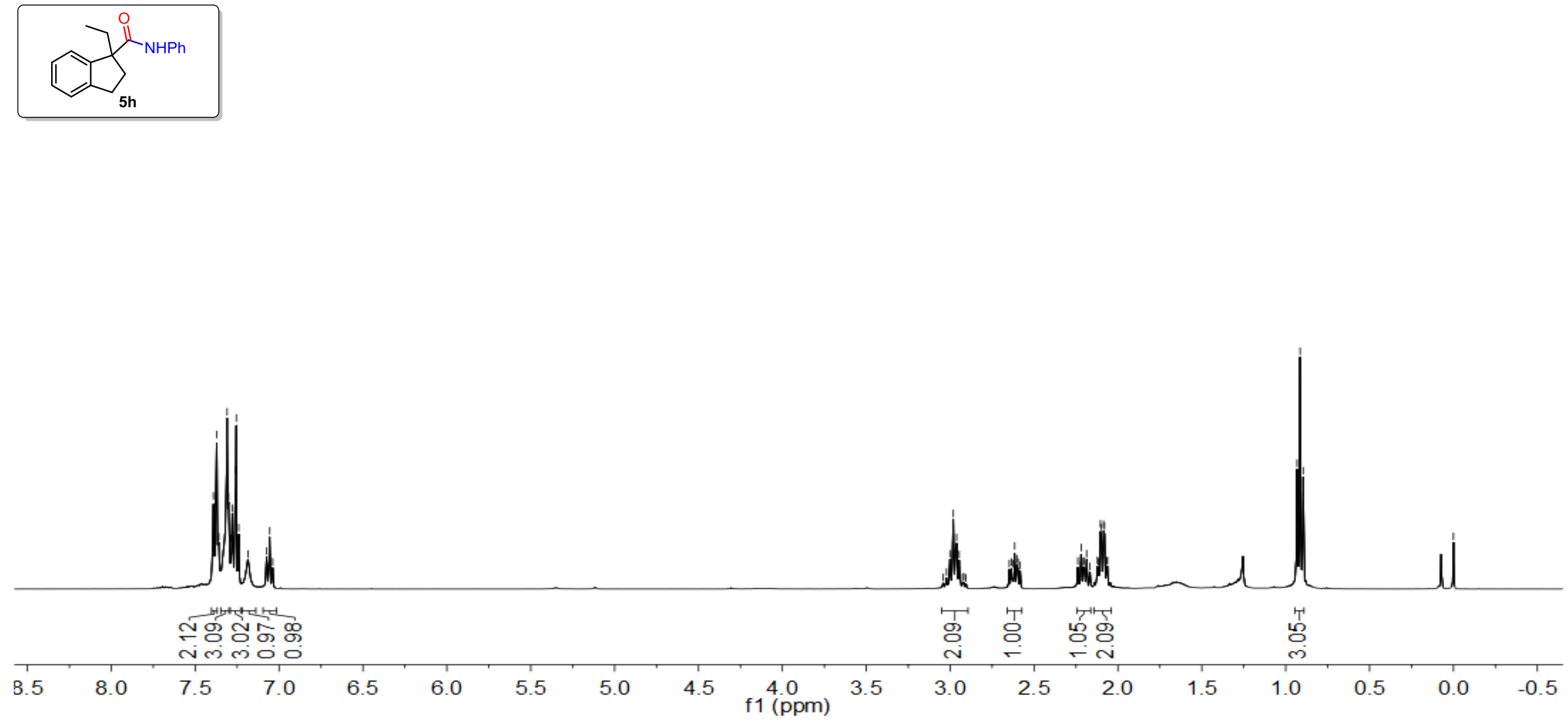
${ }^{13} \mathrm{C}$ NMR Spectra $\left(101 \mathrm{MHz}, \mathrm{CDCl}_{3}\right)$ of compound $\mathbf{5 h}$
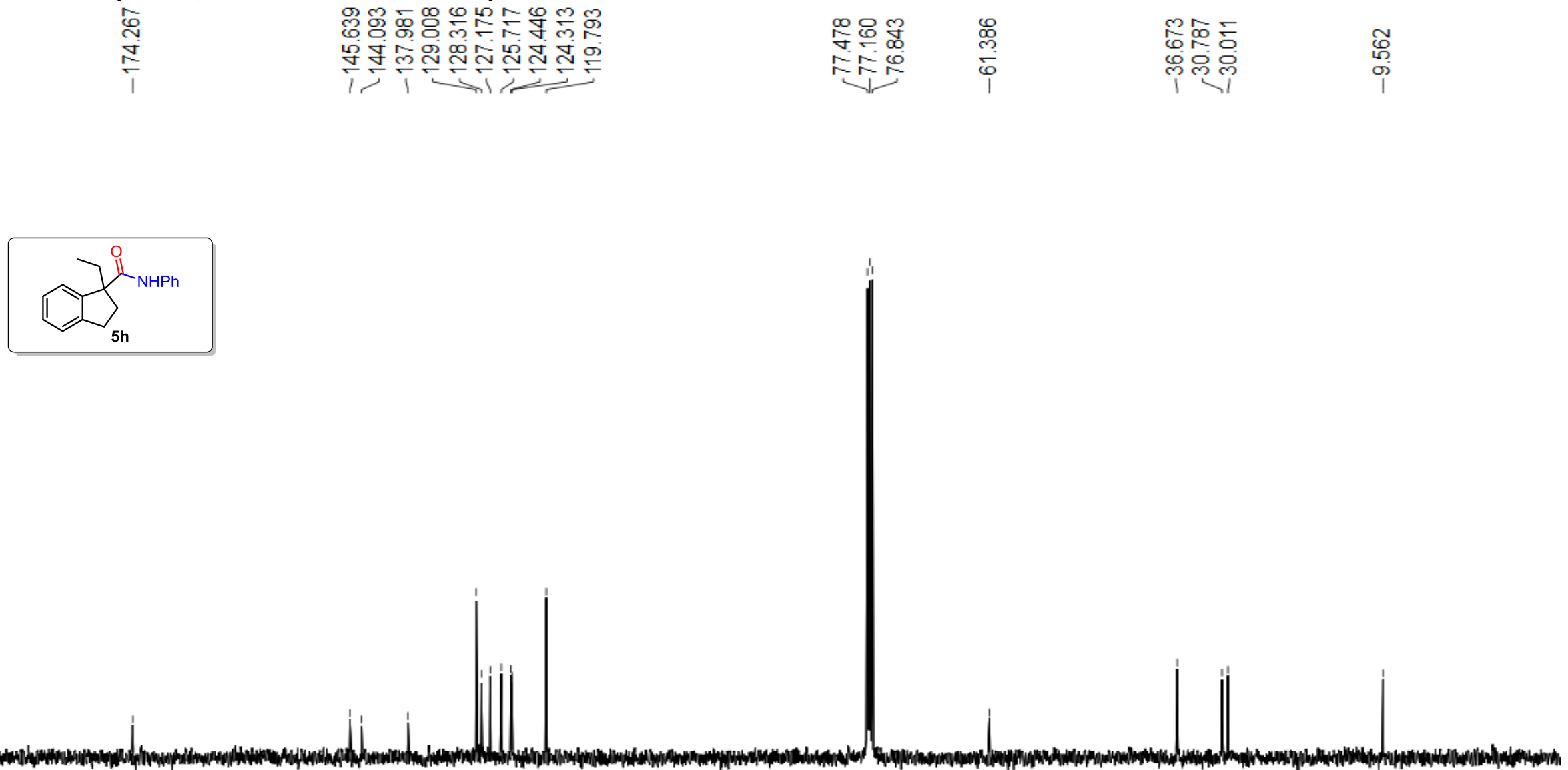

$190 \quad 180 \quad 170$

160

150

$140 \quad 130$

120

110

$100 \underset{\mathrm{f} 1(\mathrm{ppm})}{90} 80$

70

60

50

40

2010 
${ }^{1} \mathrm{H}$ NMR Spectra (600 MHz, $\mathrm{CDCl}_{3}$ ) of compound 5i

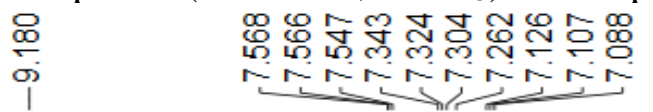

i
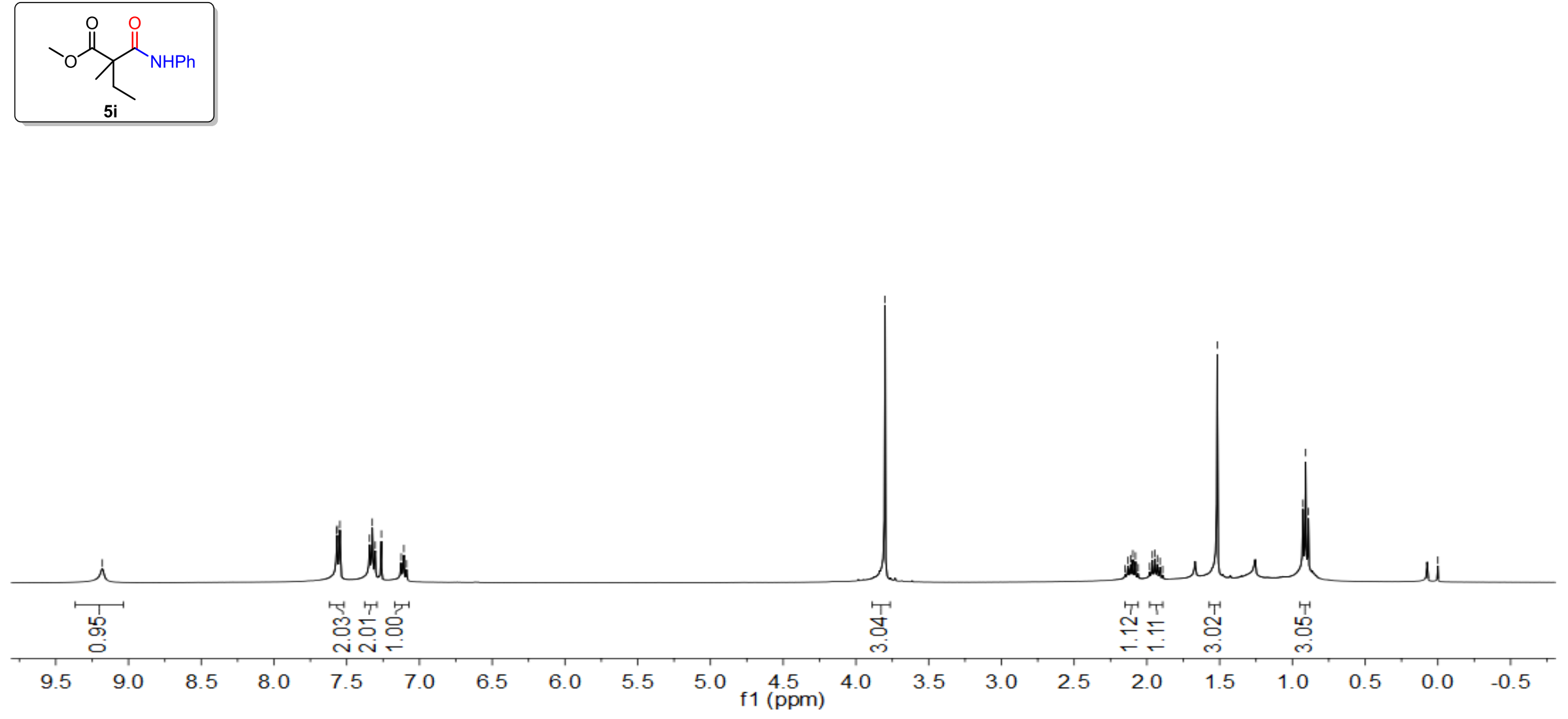
${ }^{13} \mathrm{C}$ NMR Spectra $\left(101 \mathrm{MHz}, \mathrm{CDCl}_{3}\right)$ of compound $\mathbf{5 i}$

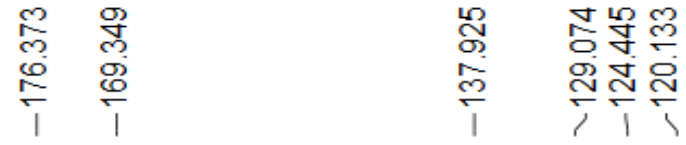

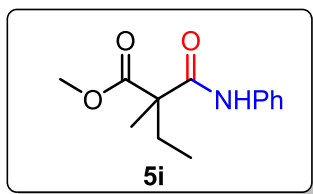

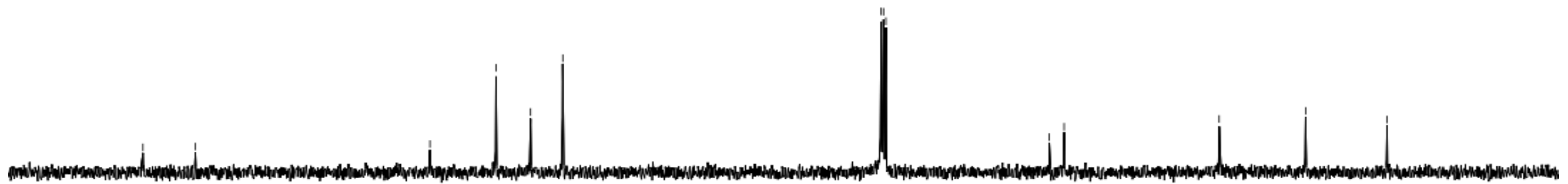

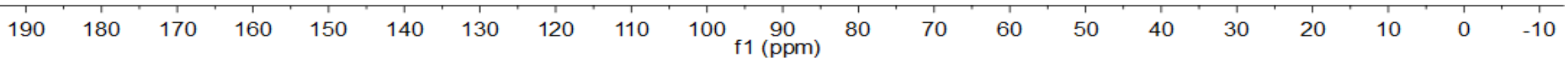


${ }^{1} \mathrm{H}$ NMR Spectra (400 MHz, $\mathrm{CDCl}_{3}$ ) of compound $\mathbf{5 j}$

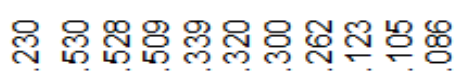

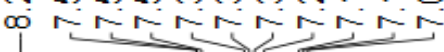
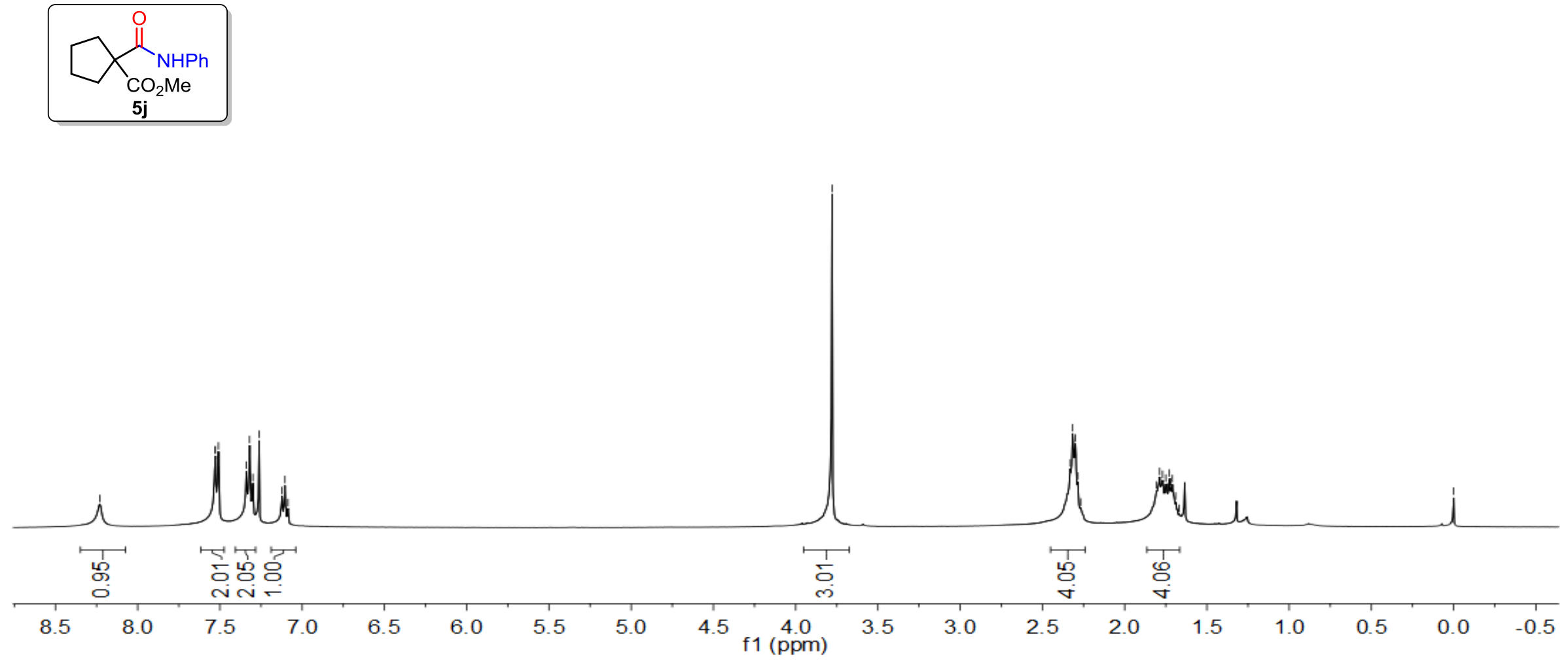
${ }^{13} \mathrm{C}$ NMR Spectra $\left(101 \mathrm{MHz}, \mathrm{CDCl}_{3}\right)$ of compound $\mathbf{5 j}$
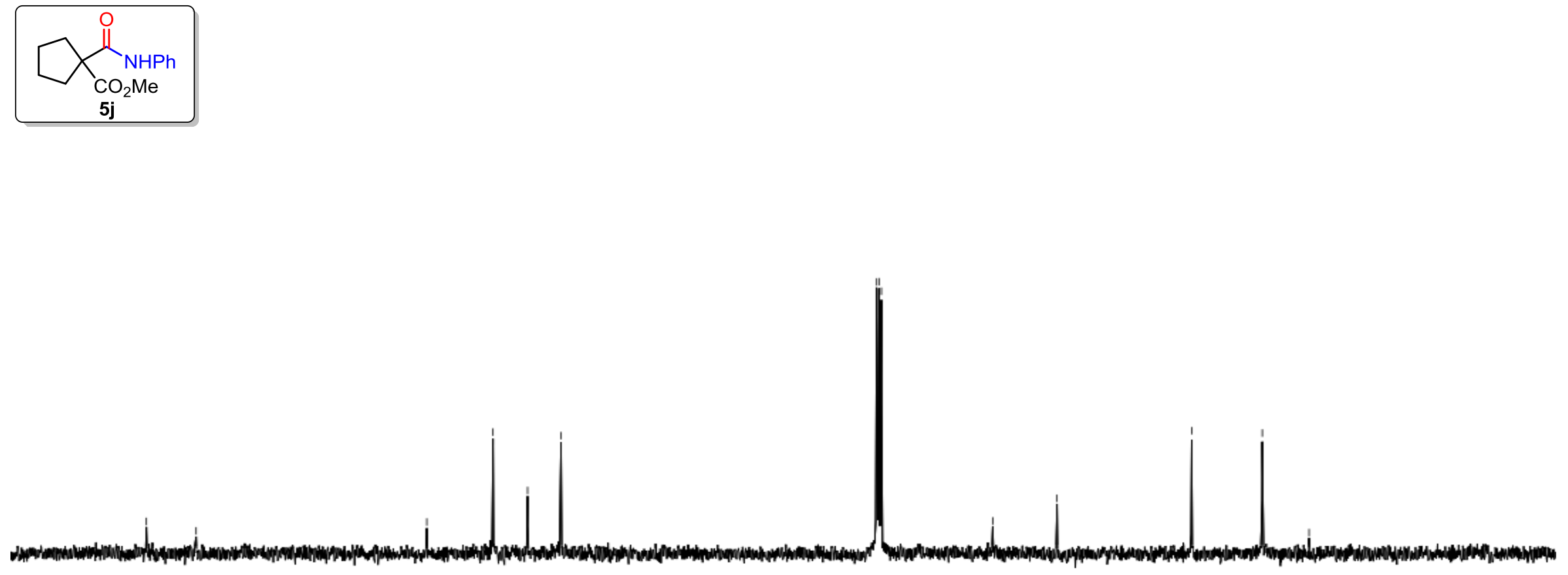

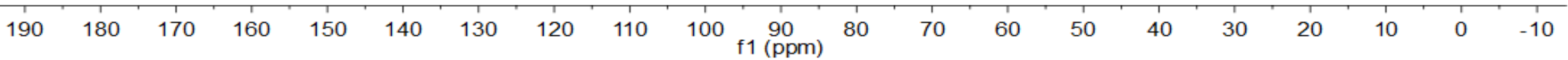


${ }^{1} \mathrm{H}$ NMR Spectra (400 MHz, $\mathrm{CDCl}_{3}$ ) of compound $\mathbf{5 k}$

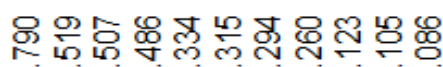

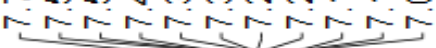
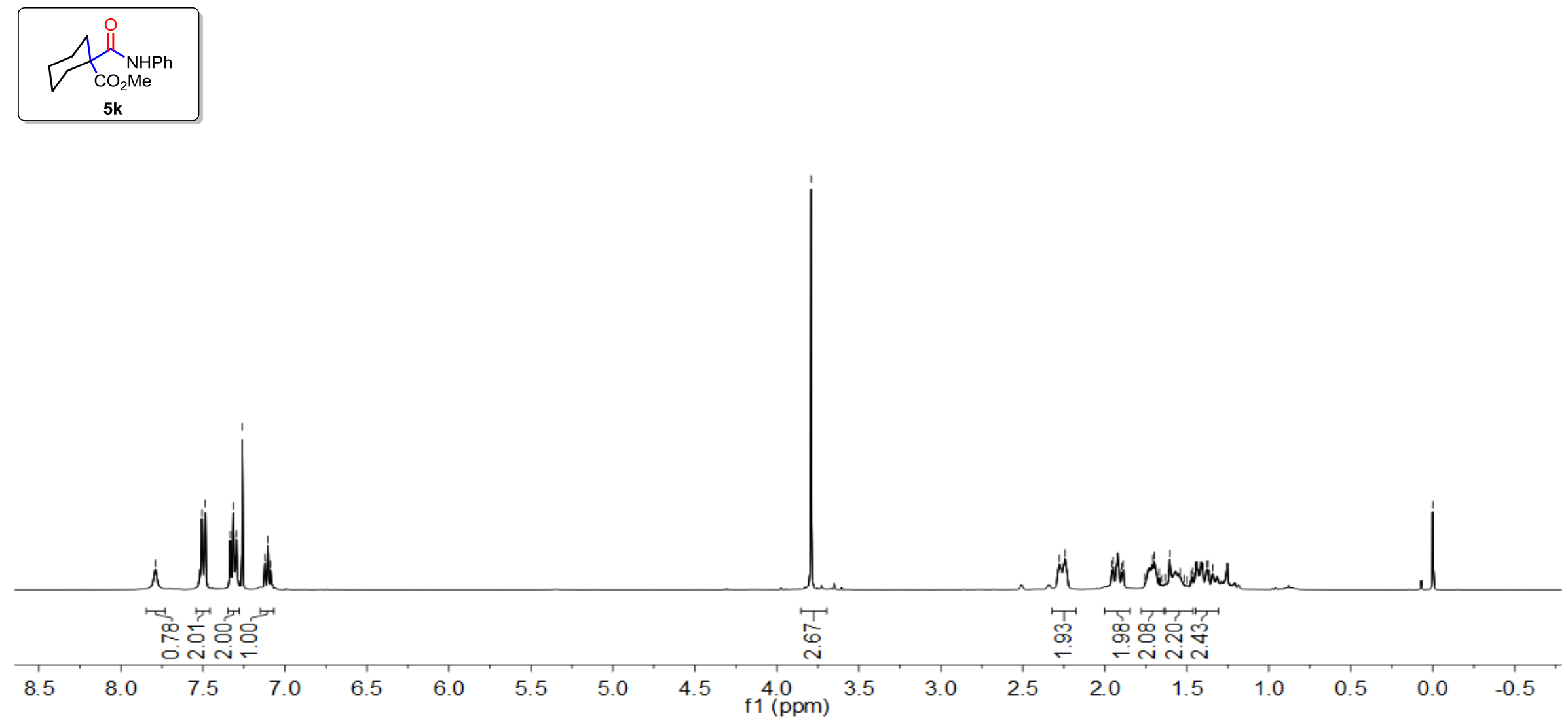
${ }^{13} \mathrm{C}$ NMR Spectra $\left(101 \mathrm{MHz}, \mathrm{CDCl}_{3}\right)$ of compound $\mathbf{5 k}$
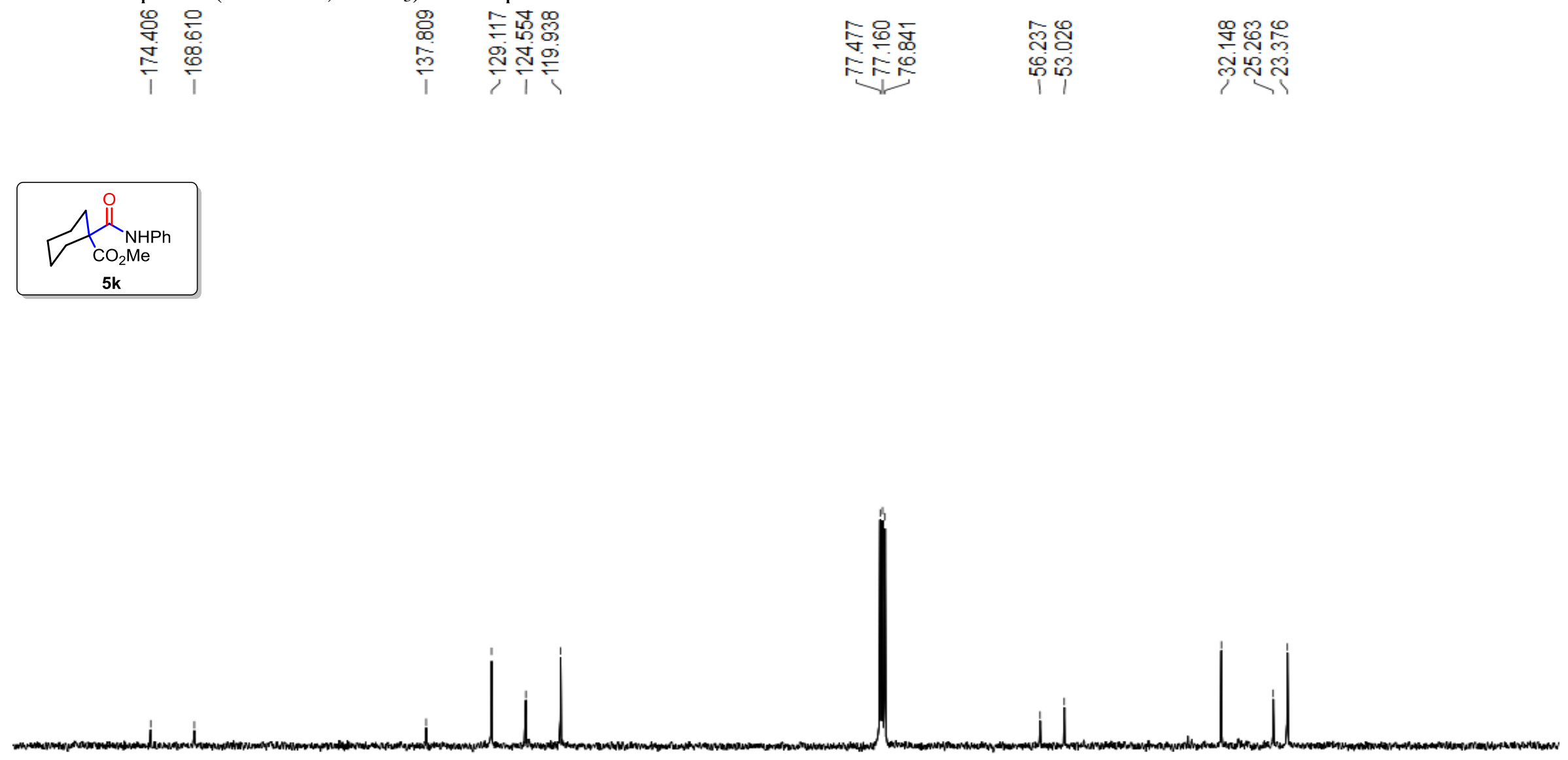
${ }^{1} \mathrm{H}$ NMR Spectra (400 MHz, $\mathrm{CDCl}_{3}$ ) of compound $\mathbf{5 l}$

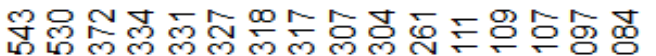

-

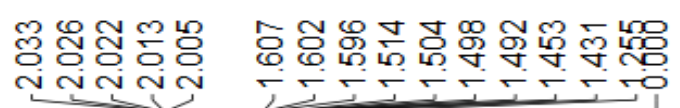
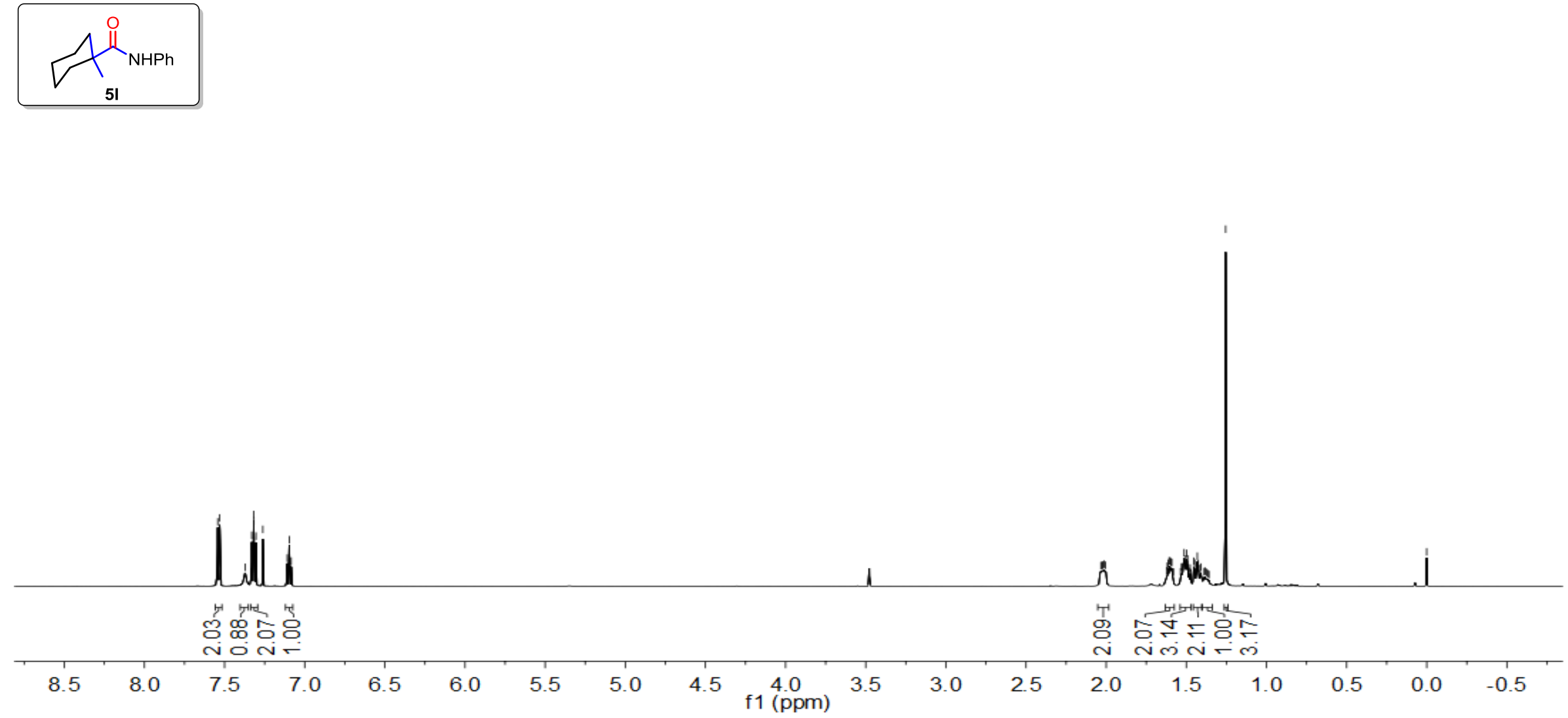
${ }^{13} \mathrm{C}$ NMR Spectra (101 MHz, $\mathrm{CDCl}_{3}$ ) of compound $\mathbf{5 l}$
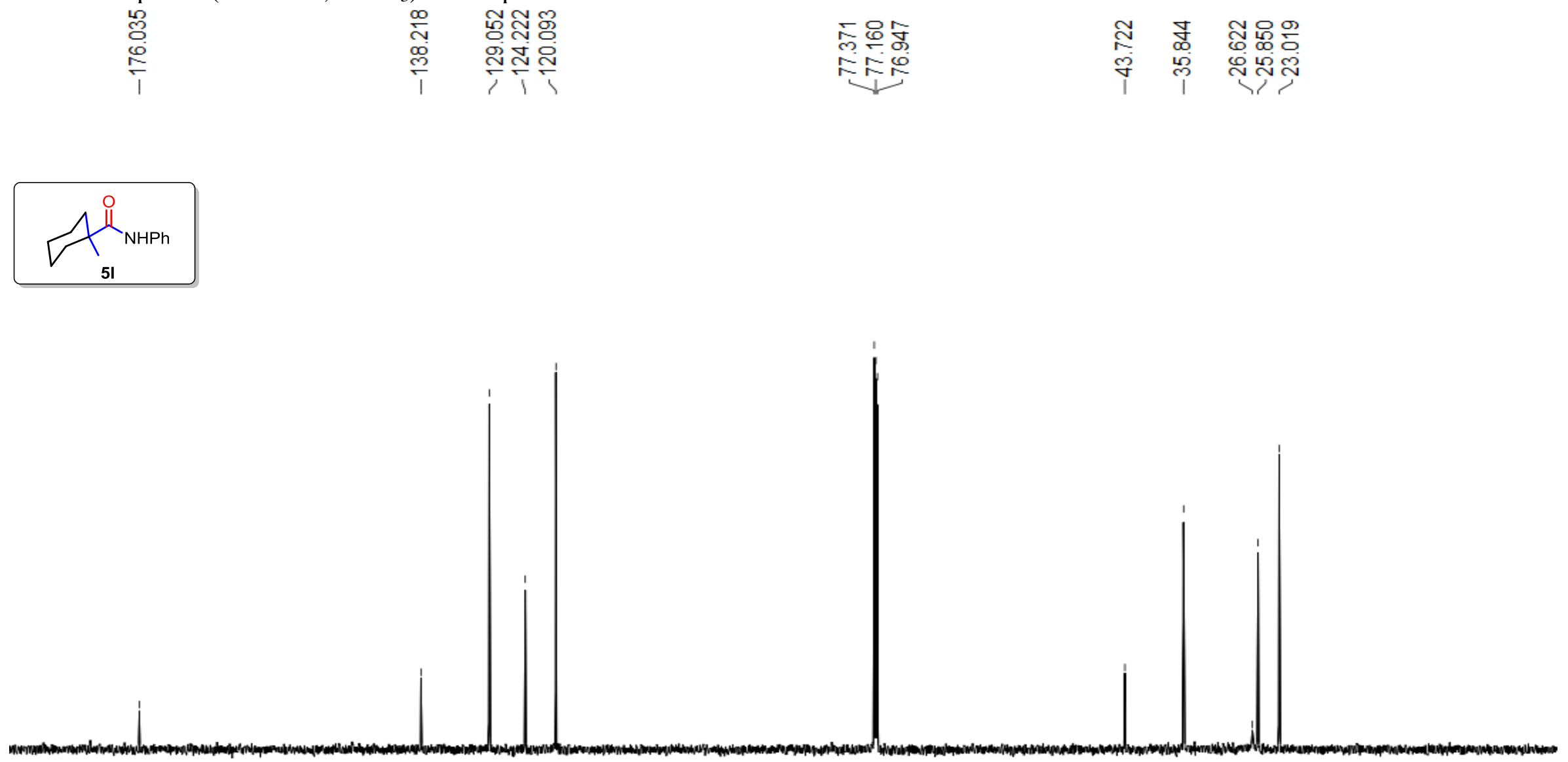

$190 \quad 180$ 
${ }^{1} \mathrm{H}$ NMR Spectra (400 MHz, $\mathrm{CDCl}_{3}$ ) of compound $\mathbf{6 a}$
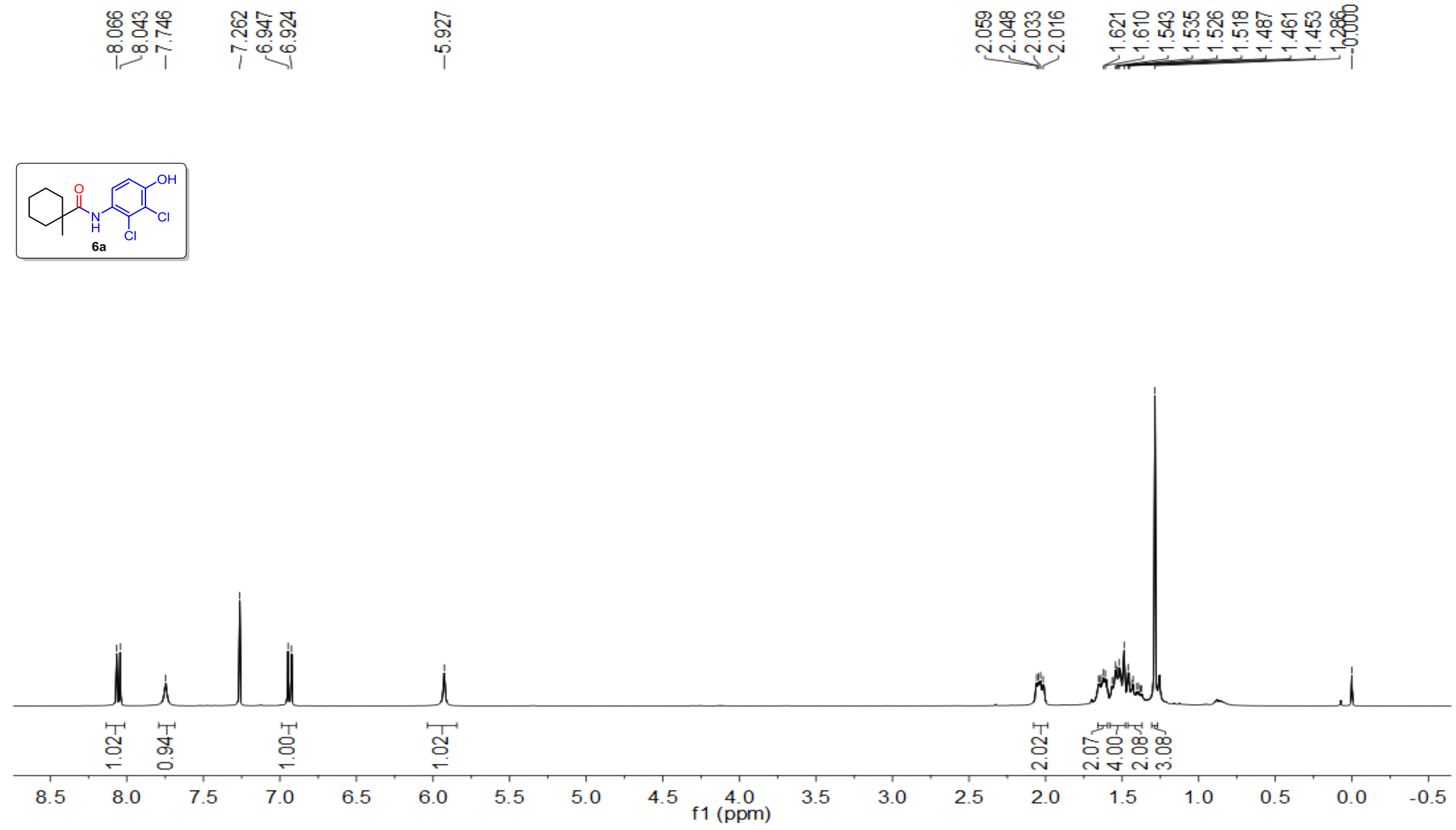
${ }^{13} \mathrm{C}$ NMR Spectra $\left(151 \mathrm{MHz}, \mathrm{CDCl}_{3}\right.$ ) of compound $\mathbf{6 a}$

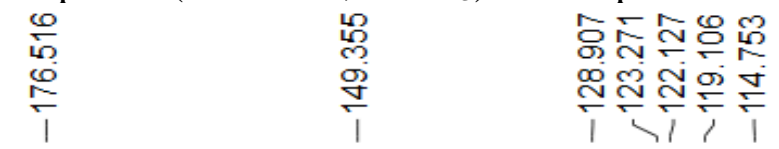

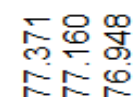

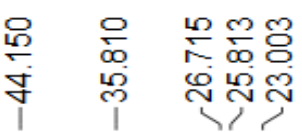

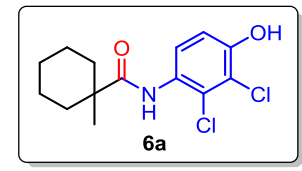

-

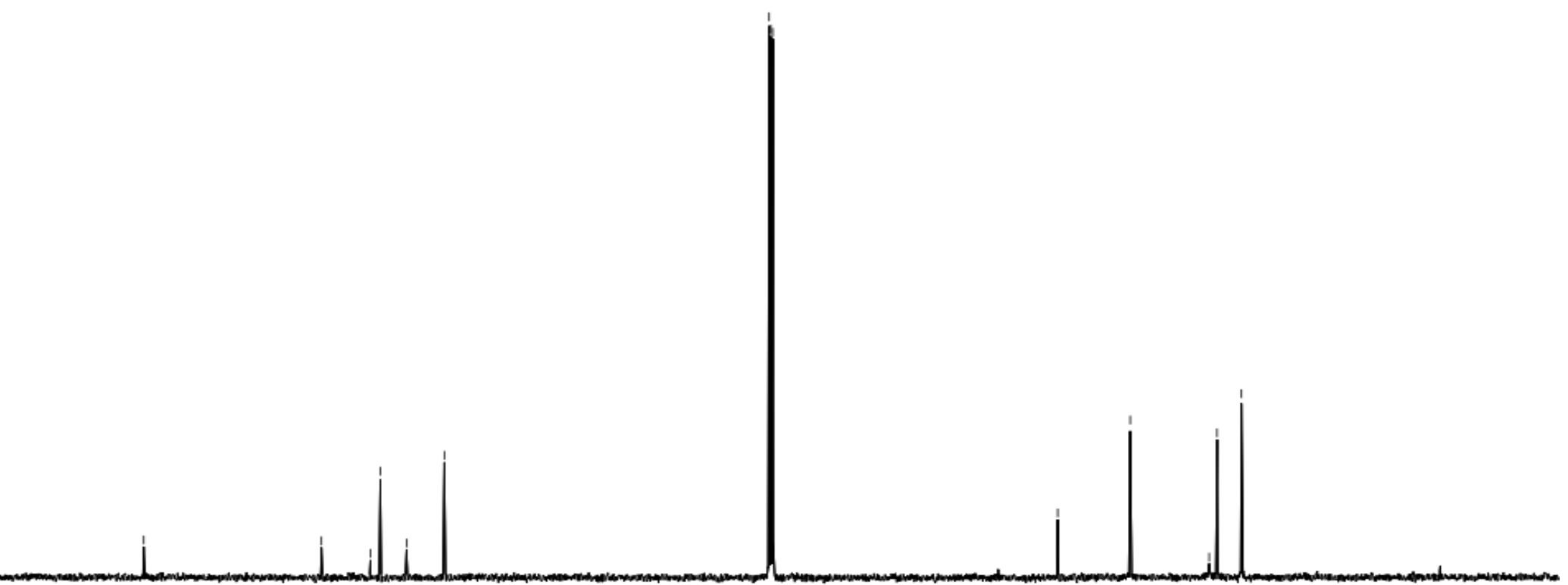

190

180

160150

$140 \quad 130$

120

110

$100 \underset{\mathrm{f} 1}{9(\mathrm{ppm})} 80$

$70 \quad 60$

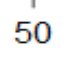

40
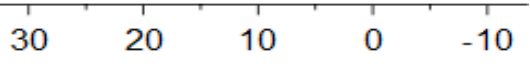
${ }^{1} \mathrm{H}$ NMR Spectra (400 MHz, $\mathrm{CDCl}_{3}$ ) of compound $\mathbf{6 b}$

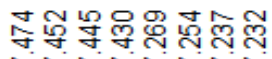

武iNan
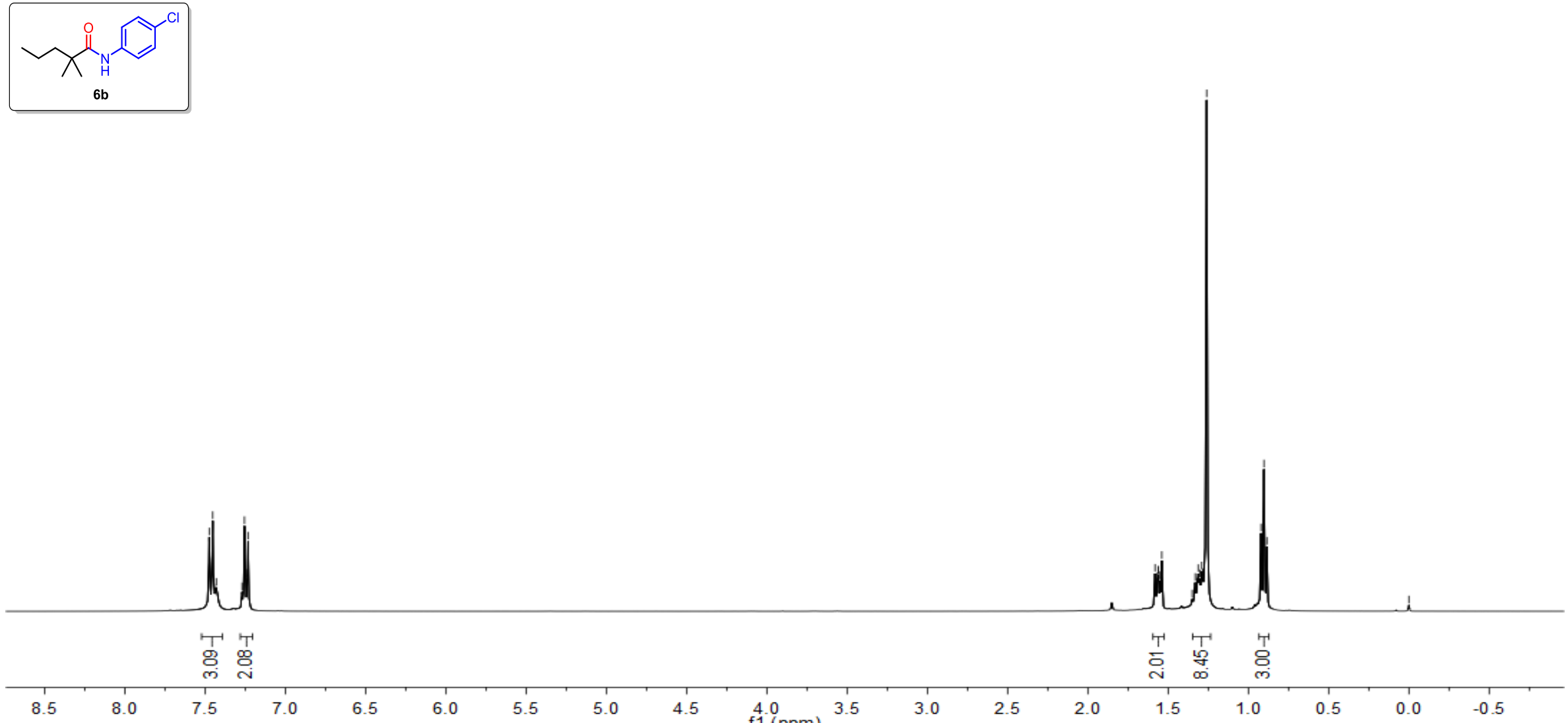
${ }^{13} \mathrm{C}$ NMR Spectra $\left(101 \mathrm{MHz}, \mathrm{CDCl}_{3}\right)$ of compound $\mathbf{6 b}$

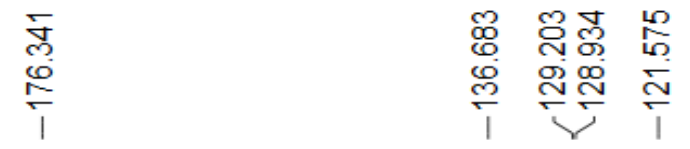

요욤

중

范㫛

守 守

กิ้ สุ๊

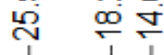
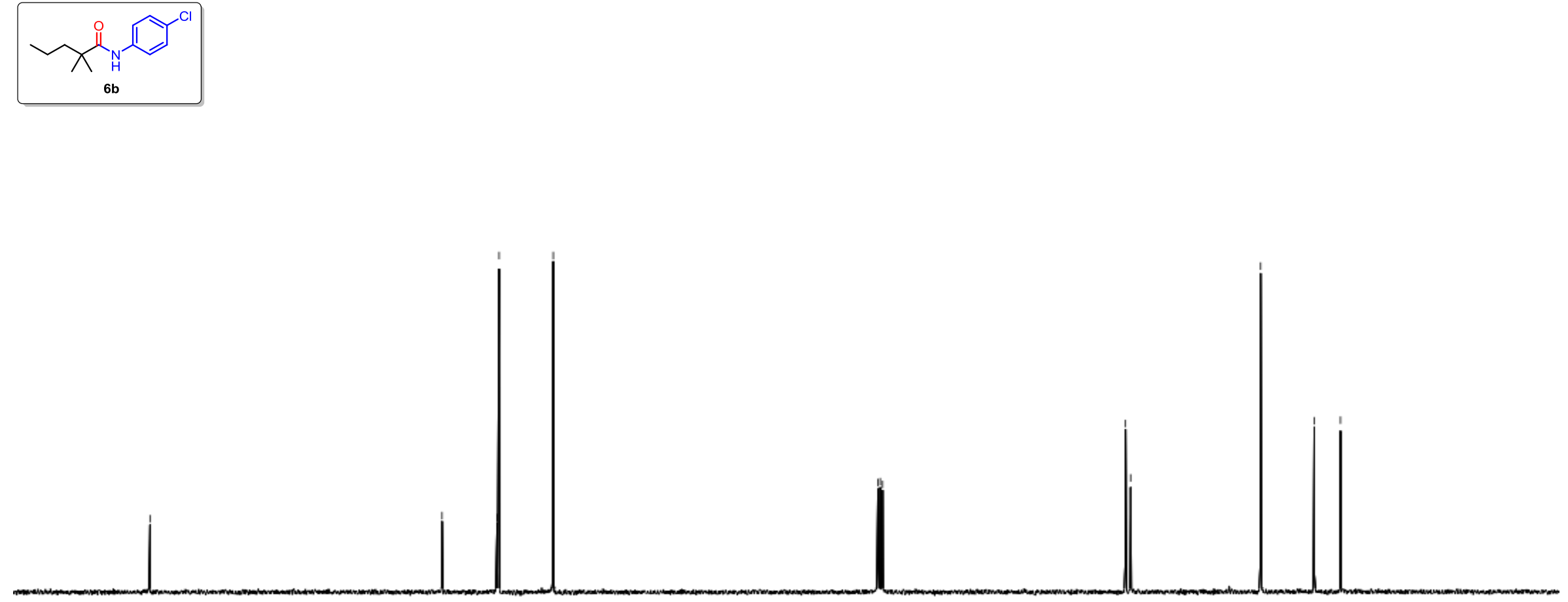

$190 \quad 180 \quad 170$

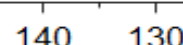

20 
${ }^{1} \mathrm{H}$ NMR Spectra (400 MHz, $\mathrm{CDCl}_{3}$ ) of compound $\mathbf{6 c}$

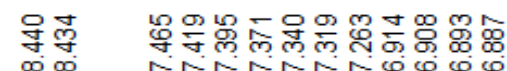

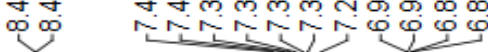
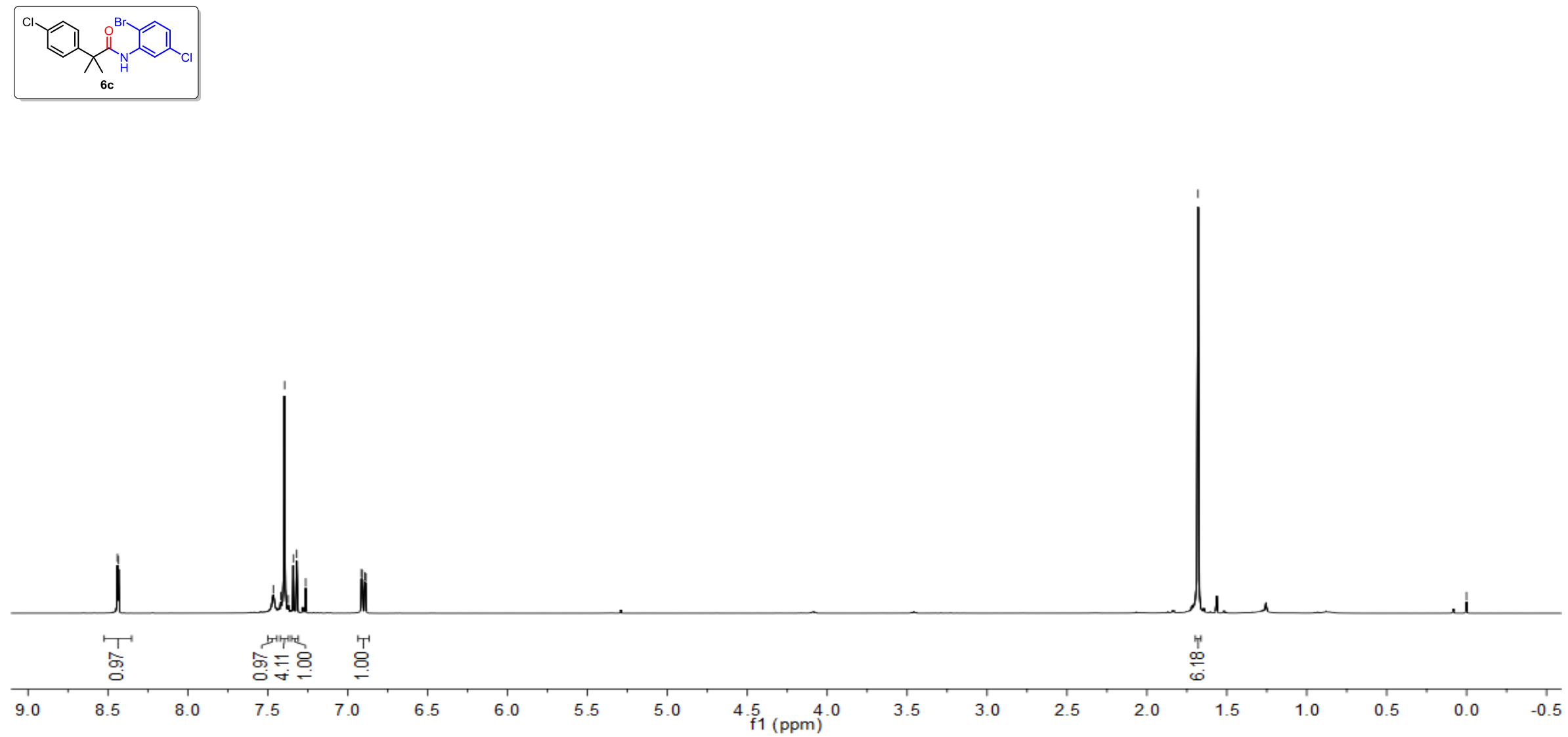

S176 
${ }^{13} \mathrm{C}$ NMR Spectra (101 MHz, $\mathrm{CDCl}_{3}$ ) of compound $\mathbf{6 c}$

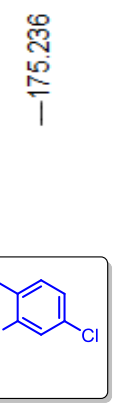

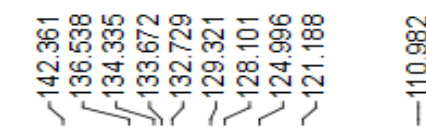

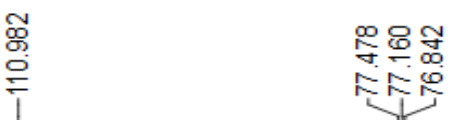

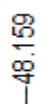

$\underset{\substack{\infty \\ i}}{\stackrel{i}{i}}$
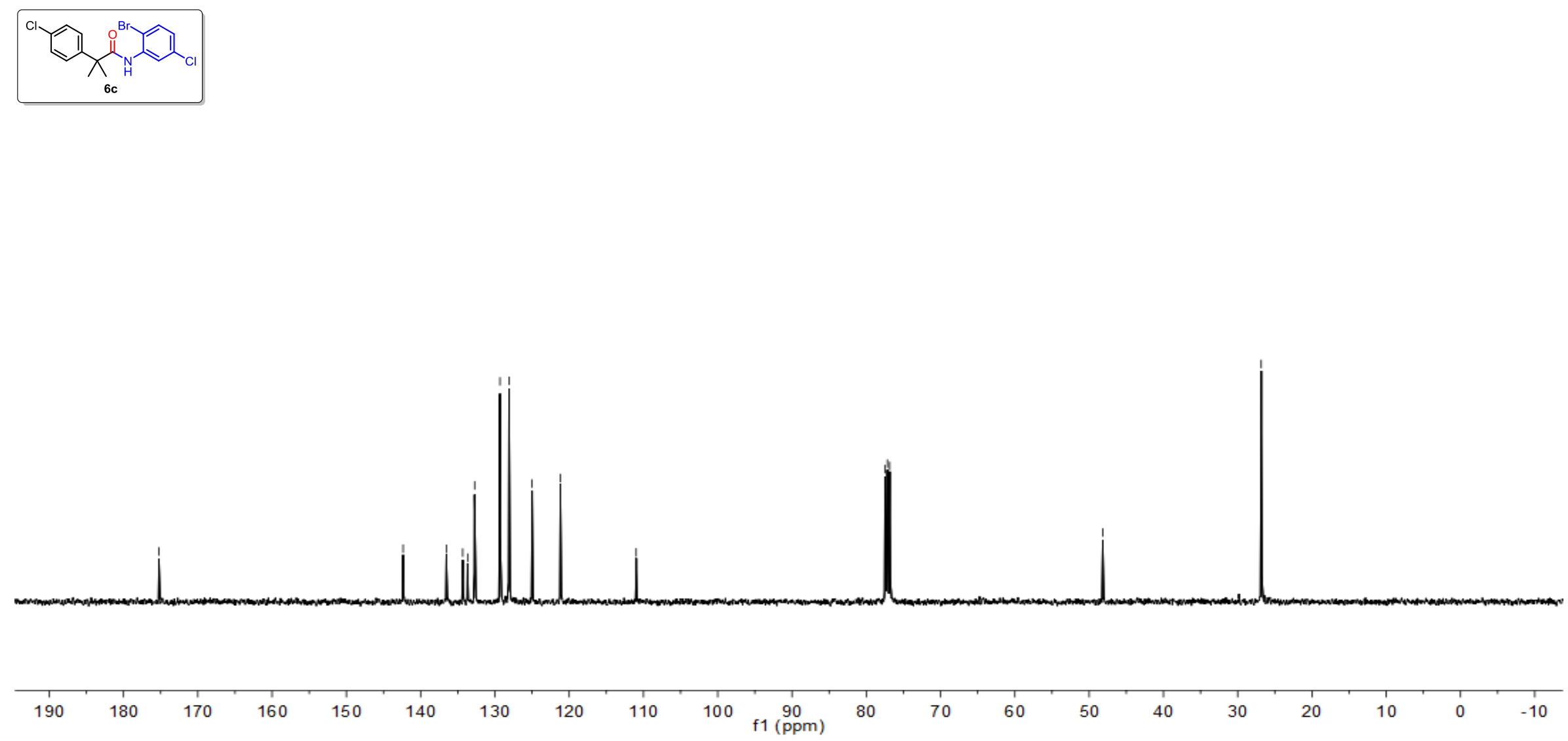
${ }^{1} \mathrm{H}$ NMR Spectra (400 MHz, $\mathrm{CDCl}_{3}$ ) of compound $\mathbf{6 d}$

م
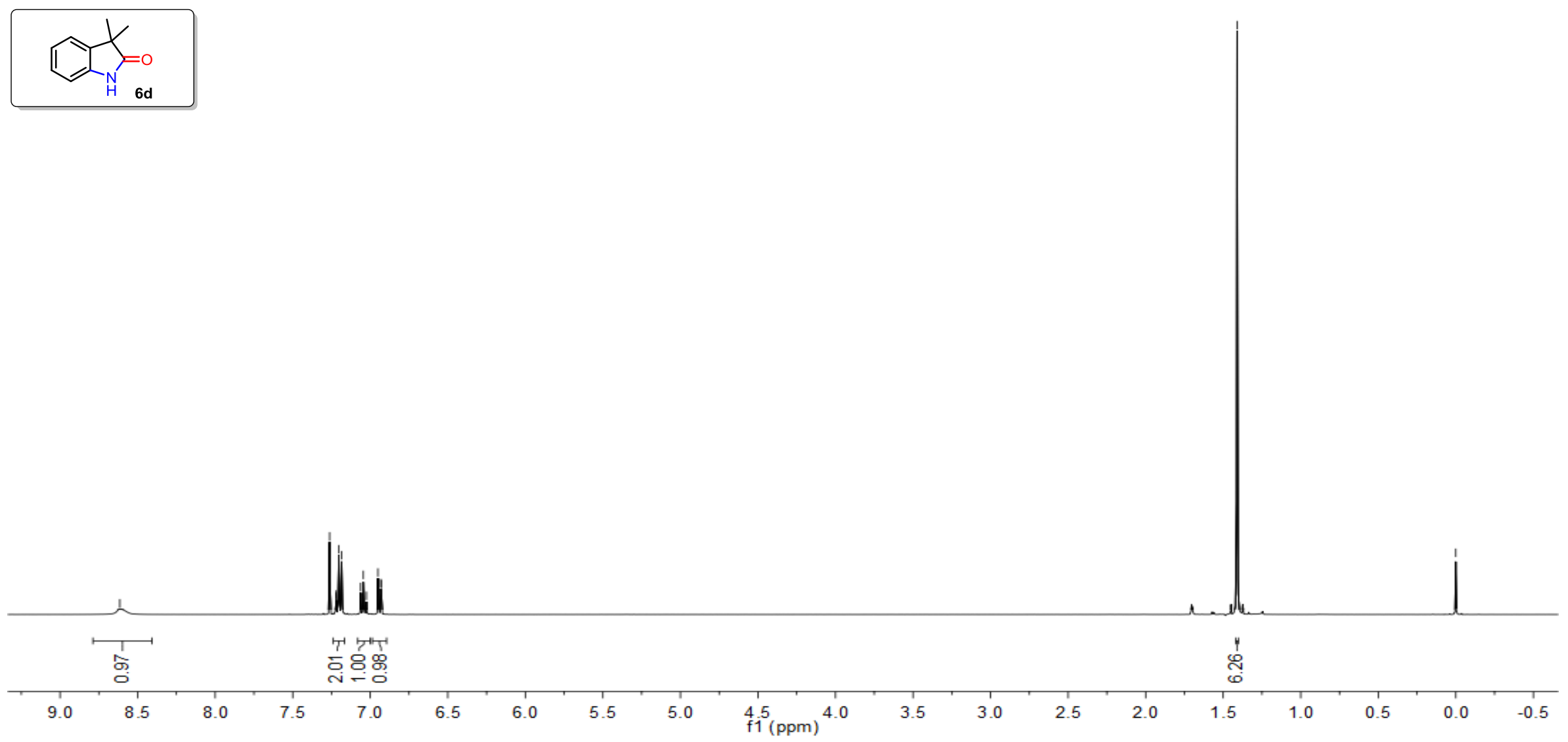
${ }^{13} \mathrm{C}$ NMR Spectra $\left(101 \mathrm{MHz}, \mathrm{CDCl}_{3}\right)$ of compound $\mathbf{6 d}$
疍

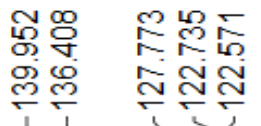
옹
テㅇํำ
숭

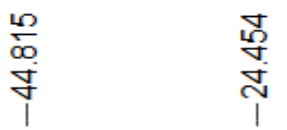
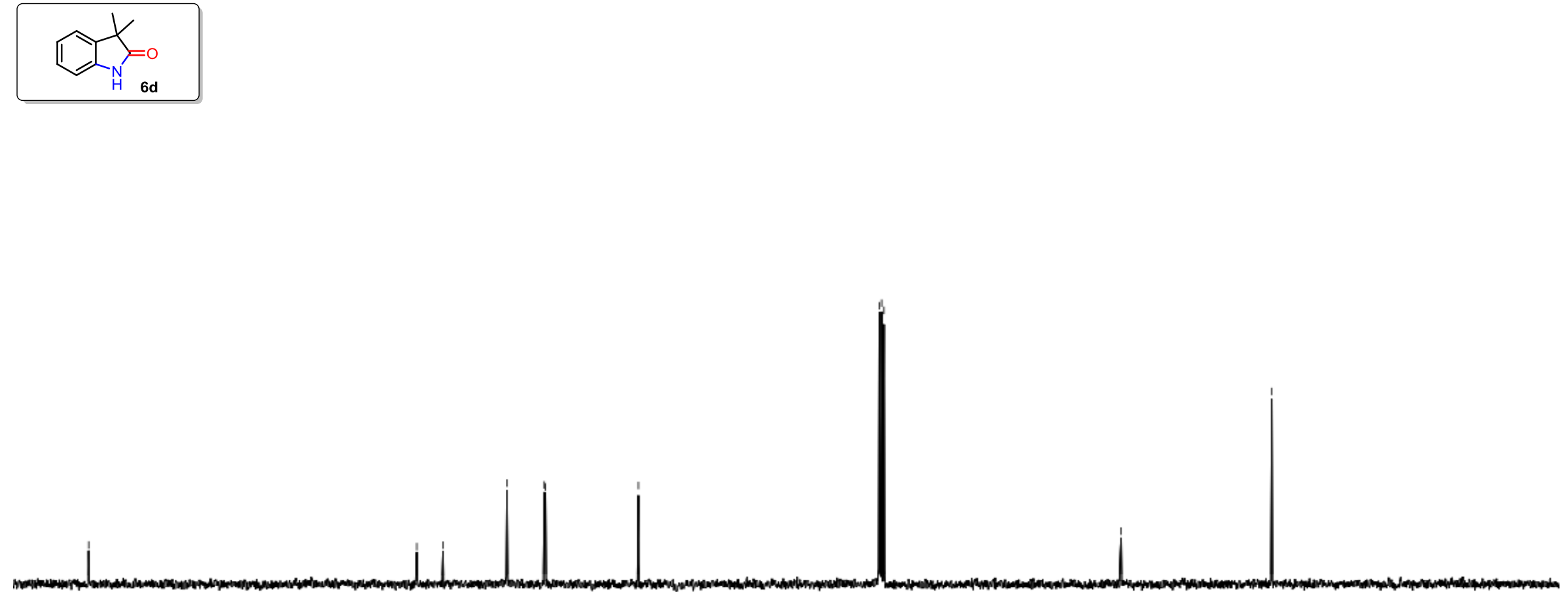

$190 \quad 180 \quad 170$

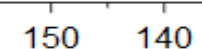

$130 \quad 120$

110

$100 \quad 90$

90

80

70

60

50

40

30

20

10

$0 \quad-10$ 